\title{
A Switchable Copper-Catalyzed Approach to Benzodithiole, Benzothiaselenole and Dibenzodithiocine Skeletons
}

Meng-Qiao Huang, ${ }^{\dagger}$ Tuan-Jie Li, ${ }^{+}$Jian-Quan Liu, ${ }^{*,+\ddagger}$ Andrey Shatskiy, ${ }^{\ddagger}$ Markus D. Kärkäs, ${ }^{*, \neq}$ Xiang-Shan Wang ${ }^{*}+$

+ School of Chemistry and Materials Science, Jiangsu Key Laboratory of Green Synthesis for Functional Materials Jiangsu Normal University, Xuzhou, Jiangsu 221116, China

‡ Department of Chemistry, KTH Royal Institute of Technology, SE-100 44 Stockholm, Sweden

* E-mail: liujq316@jsnu.edu.cn; karkas@kth.se; xswang1974@yahoo.com 


\section{Table of Contents}

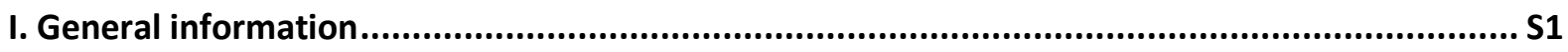

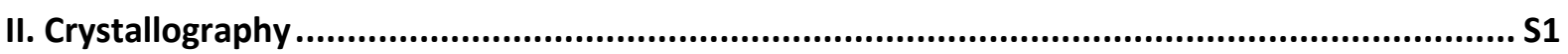

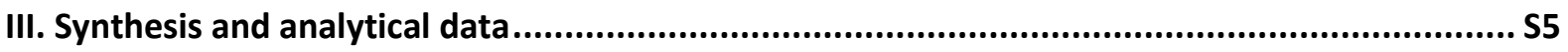

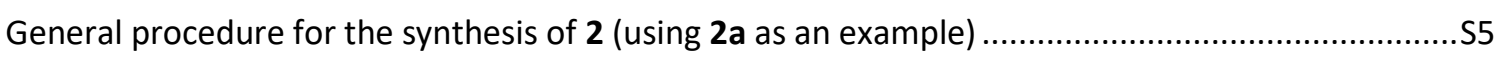

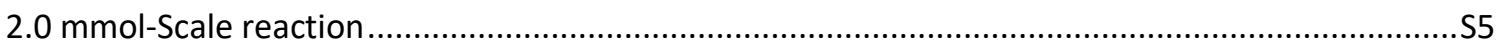

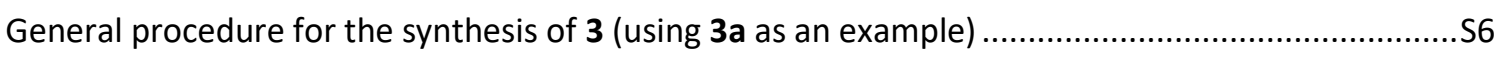

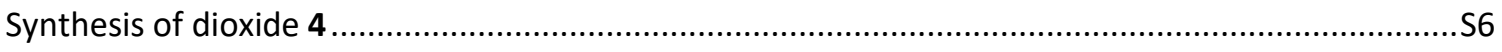

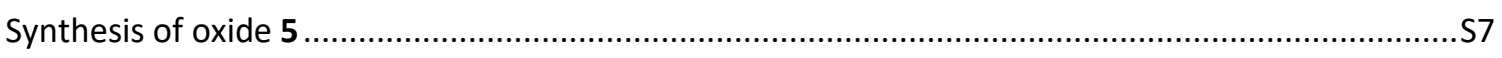

General procedure for the synthesis of $\mathbf{6}$ (using $6 \mathrm{6}$ as an example) ..................................................

General procedure for the synthesis of 7 (using $7 \mathrm{a}$ as an example) ............................................ 8

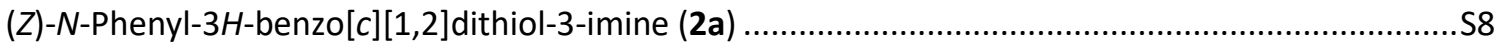

(Z)- $N$-(p-Tolyl)-3H-benzo[c] [1,2]dithiol-3-imine (2b) ...............................................................

(Z)- $N$-(4-Isopropylphenyl)-3H-benzo[c][1,2] dithiol-3-imine (2c) ...................................................

(Z)-N-(4-Methoxyphenyl)-3H-benzo[c][1,2]dithiol-3-imine (2d) ...................................................

(Z)- $\mathrm{N}$-(4-Fluorophenyl)-3H-benzo[c][1,2]dithiol-3-imine (2e) ......................................................S10

(Z)-N-(4-Chlorophenyl)-3H-benzo[c][1,2]dithiol-3-imine (2f).....................................................S10

(Z)- $\mathrm{N}$-(m-Tolyl)-3H-benzo[c][1,2]dithiol-3-imine (2g) ....................................................................

(Z)-N-(3-Chlorophenyl)-3H-benzo[c][1,2]dithiol-3-imine (2h) ....................................................S11

(Z)-N-(3-Nitrophenyl)-3H-benzo[c][1,2]dithiol-3-imine (2i) .............................................................

(Z)- $\mathrm{N}$-(o-Tolyl)-3H-benzo[c][1,2]dithiol-3-imine (2j) ....................................................................

(Z)-N-(2-Ethoxyphenyl)-3H-benzo[c][1,2]dithiol-3-imine (2k) .......................................................S12

(Z)- $\mathrm{N}$-(2-Chlorophenyl)-3H-benzo[c][1,2]dithiol-3-imine (2I) .....................................................S13

(Z)- $\mathrm{N}$-(2,4-Dichlorophenyl)-3H-benzo[c][1,2]dithiol-3-imine (2m) .............................................S13

(Z)-N-(3,4-Dimethoxyphenyl)-3H-benzo[c][1,2] dithiol-3-imine (2n) ............................................S13

(Z)- $N$-(Pyridin-2-yl)-3H-benzo[c][1,2] dithiol-3-imine (20) ................................................................

(Z)-N-(9H-Fluoren-2-yl)-3H-benzo[c][1,2]dithiol-3-imine (2p) .....................................................

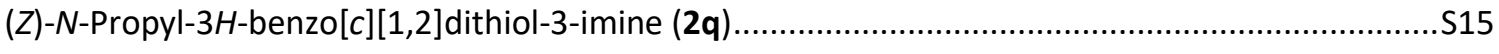

(Z)-5-Methyl-N-phenyl-3H-benzo[c][1,2]dithiol-3-imine (2r) ....................................................S15 
(Z)-5-Methyl- $N$-(p-tolyl)-3H-benzo[c][1,2]dithiol-3-imine (2s)

(Z)-N-(4-Chlorophenyl)-5-methyl-3H-benzo[c][1,2]dithiol-3-imine (2t) ....................................S16

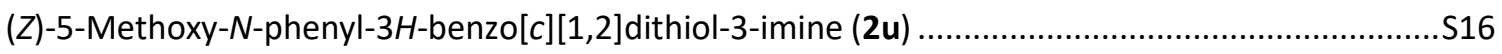

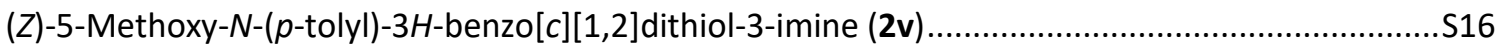

(Z)-N-(4-Chlorophenyl)-5-methoxy-3H-benzo[c][1,2] dithiol-3-imine (2w) ..............................S17

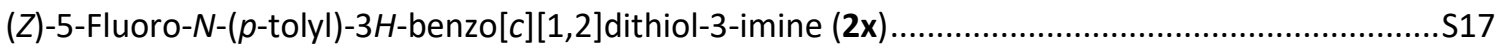

(Z)-N-(4-Chlorophenyl)-5-fluoro-3H-benzo[c][1,2]dithiol-3-imine (2y) ..................................S18

(Z)-5-Chloro-N-phenyl-3H-benzo[c][1,2]dithiol-3-imine (2z) ..............................................

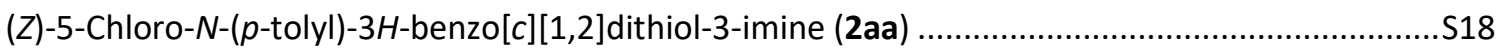

(Z)-5-Chloro- $\mathrm{N}$-(4-chlorophenyl)-3H-benzo[c][1,2]dithiol-3-imine (2ab) ..................................S19

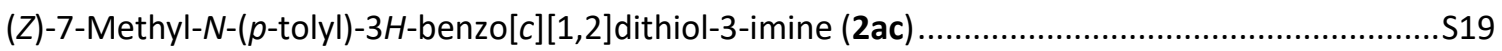

(Z)-N-(4-Chlorophenyl)-7-methyl-3H-benzo[c][1,2]dithiol-3-imine (2ad) ................................S20

(Z)-N-(4-Chlorophenyl)-3H-[1,2]dithiolo[4,3-b]pyridin-3-imine (2ae) .......................................S20

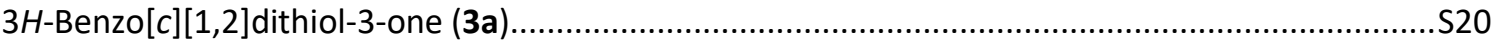

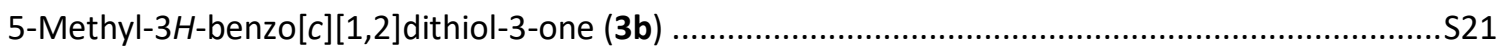

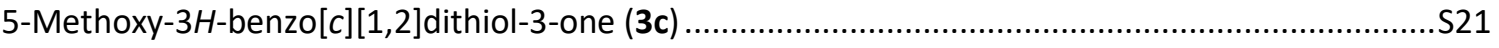

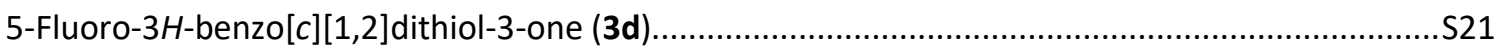

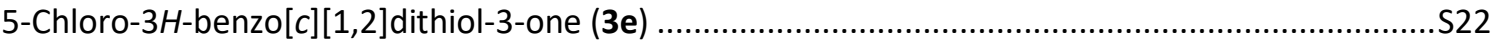

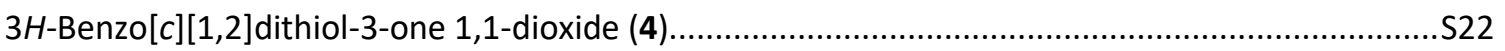

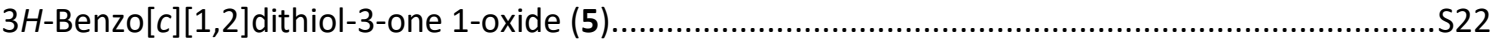

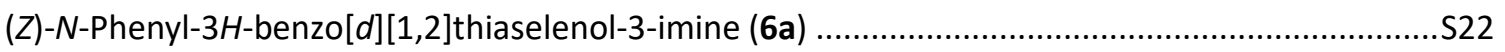

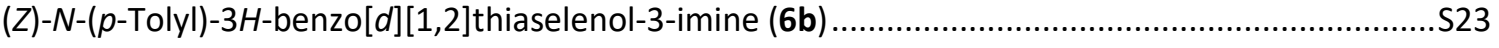

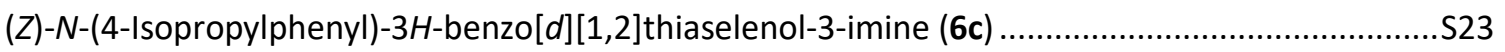

(Z)-N-(4-(tert-Butyl)phenyl)-3H-benzo[d][1,2]thiaselenol-3-imine (6d) ...................................S24

(Z)-N-(4-Methoxyphenyl)-3H-benzo[d][1,2]thiaselenol-3-imine (6e) .....................................S24

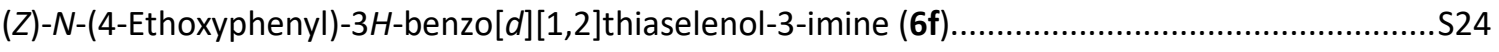

(Z)-N-(4-Chlorophenyl)-3H-benzo[d][1,2]thiaselenol-3-imine (6g) ......................................S25

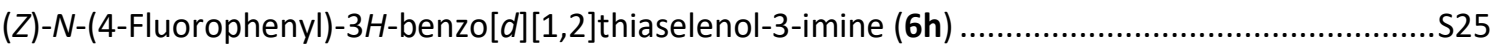

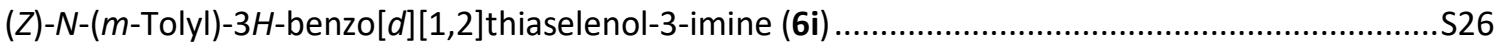

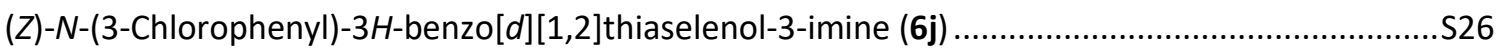


(Z)-N-(o-Tolyl)-3H-benzo[d][1,2]thiaselenol-3-imine (6k) S26

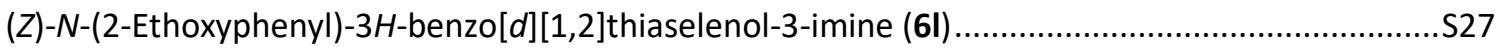

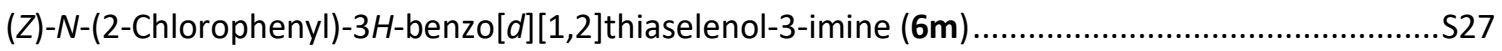

(Z)-N-(3,4-Dimethoxyphenyl)-3H-benzo[ $d][1,2]$ thiaselenol-3-imine $(6 n)$..................................S28

(Z)- $N$-(3,4-Dichlorophenyl)-3H-benzo[d][1,2]thiaselenol-3-imine (6o) .....................................S28

(Z)-N-(2,4-Dichlorophenyl)-3H-benzo[d][1,2]thiaselenol-3-imine (6p) ....................................S28

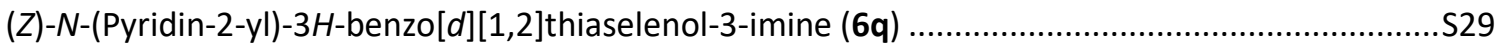

(Z)-5-Methyl-N-phenyl-3H-benzo[d][1,2]thiaselenol-3-imine (6r) ........................................S29

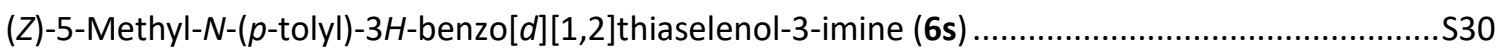

(Z)-N-(4-Chlorophenyl)-5-methyl-3H-benzo[d][1,2]thiaselenol-3-imine (6t) .............................S30

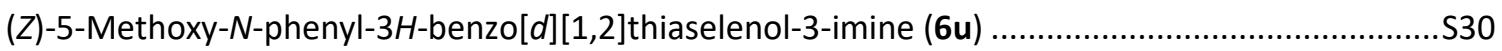

(Z)-5-Methoxy- $N$-( $p$-tolyl)-3H-benzo[d][1,2]thiaselenol-3-imine (6v) ......................................S31

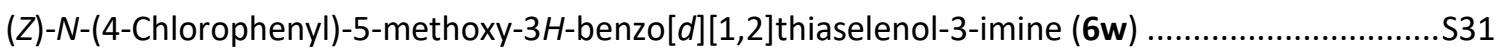

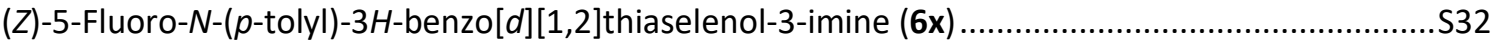

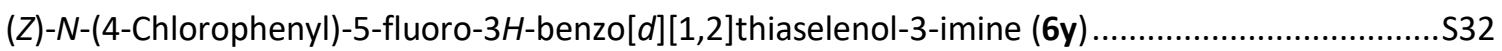

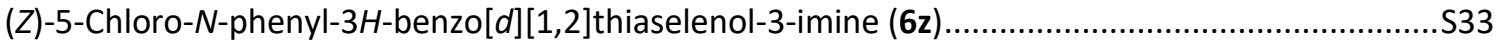

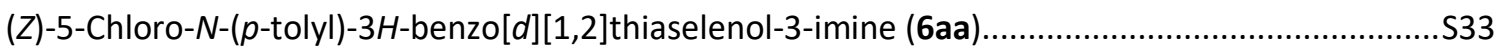

(Z)-5-Chloro- $N$-(4-chlorophenyl)-3H-benzo[d][1,2]thiaselenol-3-imine (6ab) ............................S33

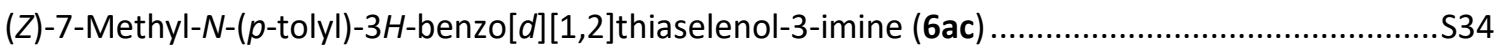

(Z)- $N$-(4-Chlorophenyl)-7-methyl-3H-benzo[d][1,2]thiaselenol-3-imine (6ad) ..........................S34

$(6 Z, 12 Z)-N^{6}, N^{12}$-Diphenyl-6H,12H-dibenzo[ $\left.b, f\right][1,5]$ dithiocine-6,12-diimine (7a) .........................S35

$(6 Z, 12 Z)-N^{6}, N^{12}$-Di-p-tolyl-6H,12H-dibenzo[ $\left.b, f\right][1,5]$ dithiocine-6,12-diimine (7b) .........................S35

$(6 Z, 12 Z)-N^{6}, N^{12}$-Bis(4-methoxyphenyl)-6H,12H-dibenzo[ $\left.b, f\right][1,5]$ dithiocine-6,12-diimine (7c)........S35

$(6 Z, 12 Z)-N^{6}, N^{12}$-Bis(4-fluorophenyl)-6H,12H-dibenzo[ $\left.b, f\right][1,5]$ dithiocine-6,12-diimine (7d)...........S36

$(6 Z, 12 Z)-N^{6}, N^{12}$-Bis(4-chlorophenyl)-6H,12H-dibenzo[ $\left.b, f\right][1,5]$ dithiocine-6,12-diimine (7e) ...........S36

$(6 Z, 12 Z)-N^{6}, N^{12}$-Di- $m$-tolyl-6H,12H-dibenzo[ $\left.b, f\right][1,5]$ dithiocine-6,12-diimine (7f)........................S37

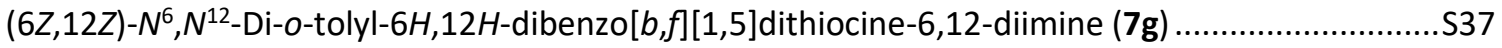

$(6 Z, 12 Z)-N^{6}, N^{12}$-Bis(3-chlorophenyl)-6H,12H-dibenzo[b,f][1,5]dithiocine-6,12-diimine (7h) ...........S37

$(6 Z, 12 Z)-N^{6}, N^{12}$-Bis(2-chlorophenyl)-6H,12H-dibenzo[b,f][1,5]dithiocine-6,12-diimine (7i) ............S38

$(6 Z, 12 Z)-2,8$-Dimethyl- $N^{6}, N^{12}$-diphenyl-6H,12H-dibenzo[b,f][1,5]dithiocine-6,12-diimine (7j) ........S38 
$(6 Z, 12 Z)-2,8$-Dimethyl- $N^{6}, N^{12}$-di-p-tolyl-6H,12H-dibenzo[b,f][1,5]dithiocine-6,12-diimine (7k) S39

$(6 Z, 12 Z)-N^{6}, N^{12}$-Bis(4-chlorophenyl)-2,8-dimethyl-6H,12H-dibenzo[ $\left.b, f\right][1,5]$ dithiocine-6,12-diimine

(7I). S39

(6Z,12Z)-2,8-Dimethoxy- $N^{6}, N^{12}$-diphenyl-6H,12H-dibenzo[ $\left.b, f\right][1,5]$ dithiocine-6,12-diimine (7m)....S39

$(6 Z, 12 Z)-2,8$-Dimethoxy- $N^{6}, N^{12}$-di-p-tolyl-6H,12H-dibenzo[b,f][1,5]dithiocine-6,12-diimine (7n) ....S40 $(6 Z, 12 Z)-N^{6}, N^{12}$-Bis(4-chlorophenyl)-2,8-dimethoxy-6H,12H-dibenzo[ $\left.b, f\right][1,5]$ dithiocine-6,12-diimine (7o) S40

(6Z,12Z)-2,8-Dichloro- $N^{6}, N^{12}$-diphenyl-6H,12H-dibenzo[b,f][1,5] dithiocine-6,12-diimine (7p) S41

(6Z,12Z)-2,8-Dichloro- $N^{6}, N^{12}$-di-p-tolyl-6H,12H-dibenzo[ $\left.b, f\right][1,5]$ dithiocine-6,12-diimine (7q) S41

(6Z,12Z)-2,8-Dichloro- $N^{6}, N^{12}$-bis(4-chlorophenyl)-6H,12H-dibenzo[b,f][1,5]dithiocine-6,12-diimine (7r)

$(6 Z, 12 Z)-4,10$-Dimethyl- $N^{6}, N^{12}$-di-p-tolyl-6H,12H-dibenzo[ $\left.b, f\right][1,5]$ dithiocine-6,12-diimine (7s) .....S42 $(6 Z, 12 Z)-N^{6}, N^{12}$-Bis(4-chlorophenyl)-4,10-dimethyl-6H,12H-dibenzo[ $\left.b, f\right][1,5]$ dithiocine-6,12-diimine (7t) S42

IV. NMR spectra S43

(Z)-N-Phenyl-3H-benzo[c][1,2]dithiol-3-imine (2a) .543

(Z)-N-(p-Tolyl)-3H-benzo[c][1,2]dithiol-3-imine (2b). .544

(Z)- $N$-(4-Isopropylphenyl)-3H-benzo[c][1,2]dithiol-3-imine (2c) . S45

(Z)-N-(4-Methoxyphenyl)-3H-benzo[c][1,2]dithiol-3-imine (2d) . .546

(Z)-N-(4-Fluorophenyl)-3H-benzo[c][1,2]dithiol-3-imine (2e) .547

(Z)-N-(4-Chlorophenyl)-3H-benzo[c][1,2]dithiol-3-imine (2f). .548

(Z)-N-(m-Tolyl)-3H-benzo[c][1,2]dithiol-3-imine (2g) .549

(Z)-N-(3-Chlorophenyl)-3H-benzo[c][1,2]dithiol-3-imine (2h). S50

(Z)-N-(3-Nitrophenyl)-3H-benzo[c][1,2]dithiol-3-imine (2i) .S51

(Z)-N-(o-Tolyl)-3H-benzo[c][1,2]dithiol-3-imine (2j). S52

(Z)-N-(2-Ethoxyphenyl)-3H-benzo[c][1,2]dithiol-3-imine (2k) .S53

(Z)-N-(2-Chlorophenyl)-3H-benzo[c][1,2]dithiol-3-imine (2l) . S54

(Z)-N-(2,4-Dichlorophenyl)-3H-benzo[c][1,2]dithiol-3-imine (2m) .555

(Z)-N-(3,4-Dimethoxyphenyl)-3H-benzo[c][1,2] dithiol-3-imine (2n). S56

(Z)-N-(Pyridin-2-yl)-3H-benzo[c][1,2] dithiol-3-imine (2o) S57

(Z)-N-(9H-Fluoren-2-yl)-3H-benzo[c][1,2]dithiol-3-imine (2p) S58 
(Z)-N-Propyl-3H-benzo[c][1,2]dithiol-3-imine (2q)

(Z)-5-Methyl-N-phenyl-3H-benzo[c][1,2]dithiol-3-imine (2r) ...............................................S60

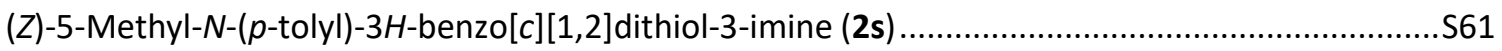

(Z)-N-(4-Chlorophenyl)-5-methyl-3H-benzo[c][1,2]dithiol-3-imine (2t) ...................................S62

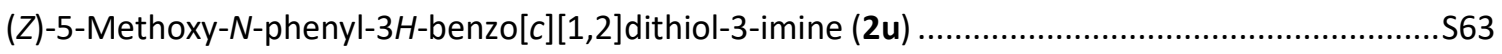

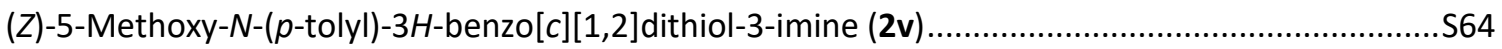

(Z)-N-(4-Chlorophenyl)-5-methoxy-3H-benzo[c][1,2]dithiol-3-imine (2w) ...............................S65

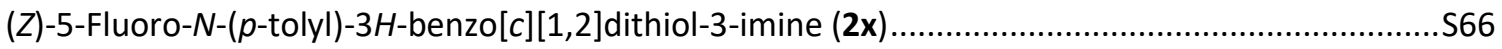

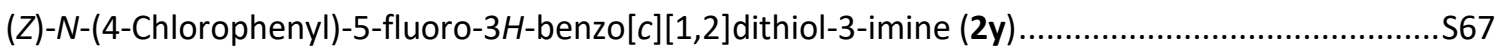

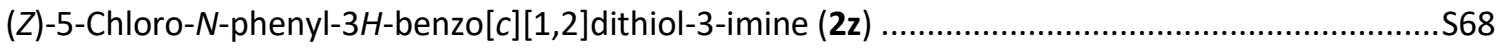

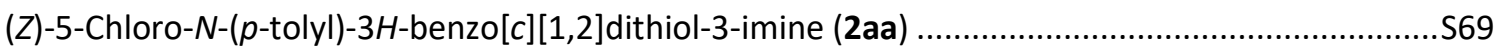

(Z)-5-Chloro- $\mathrm{N}$-(4-chlorophenyl)-3H-benzo[c][1,2]dithiol-3-imine (2ab) ..................................S70

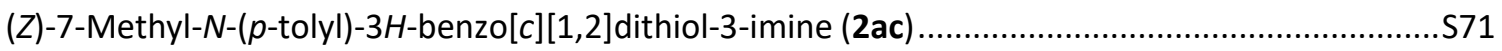

(Z)-N-(4-Chlorophenyl)-7-methyl-3H-benzo[c][1,2] dithiol-3-imine (2ad) .................................S72

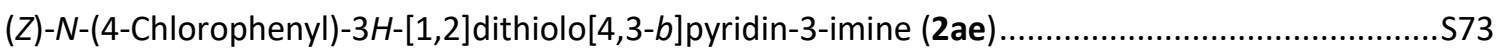

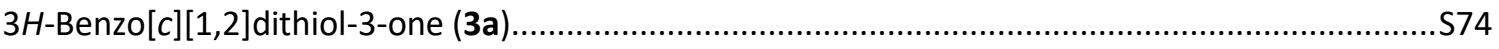

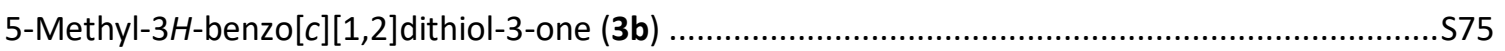

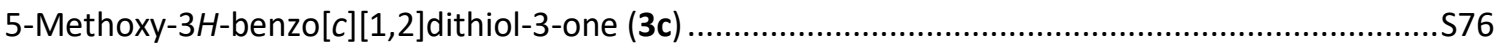

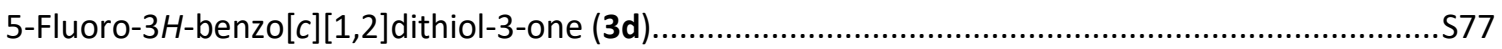

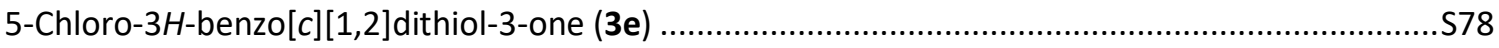

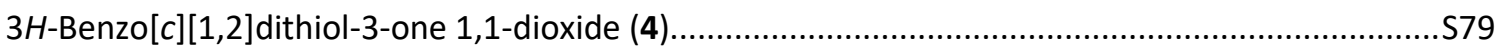

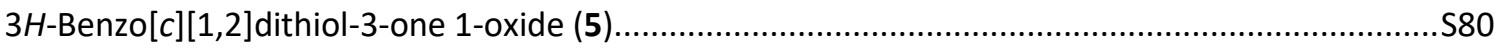

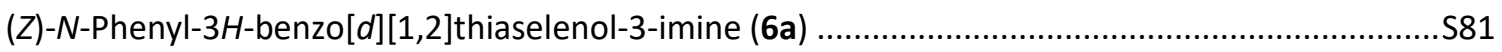

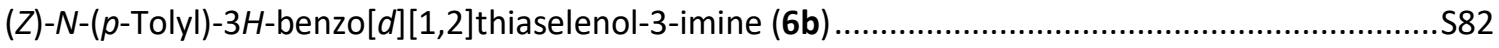

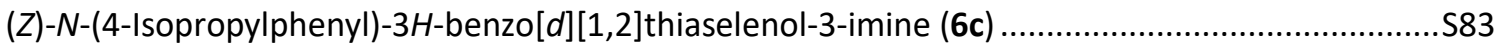

(Z)-N-(4-(tert-Butyl)phenyl)-3H-benzo[d][1,2]thiaselenol-3-imine (6d) .................................S84

(Z)-N-(4-Methoxyphenyl)-3H-benzo[d][1,2]thiaselenol-3-imine (6e) ....................................S85

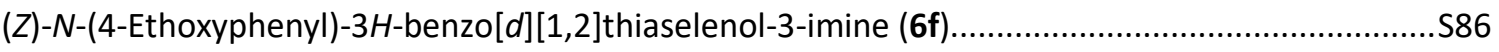

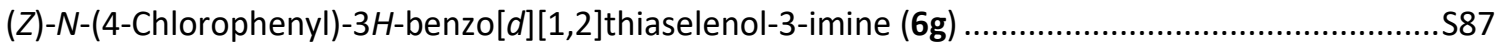

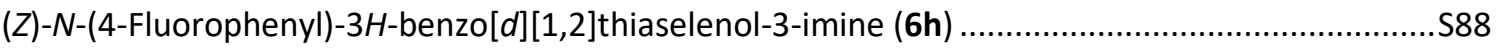


(Z)-N-(m-Tolyl)-3H-benzo[d][1,2]thiaselenol-3-imine (6i) .

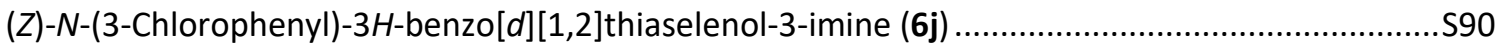

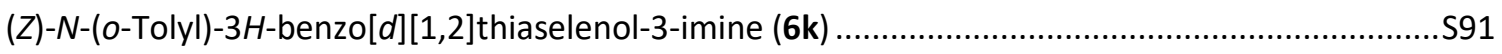

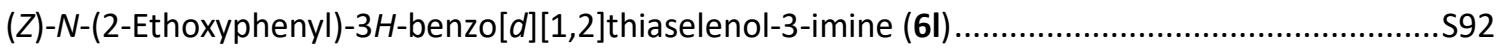

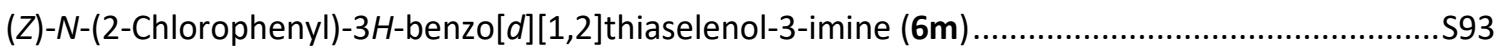

(Z)-N-(3,4-Dimethoxyphenyl)-3H-benzo[d][1,2]thiaselenol-3-imine (6n) ..................................S94

(Z)- $N$-(3,4-Dichlorophenyl)-3H-benzo[d][1,2]thiaselenol-3-imine (6o) ....................................S95

(Z)-N-(2,4-Dichlorophenyl)-3H-benzo[d][1,2]thiaselenol-3-imine (6p) ...................................S96

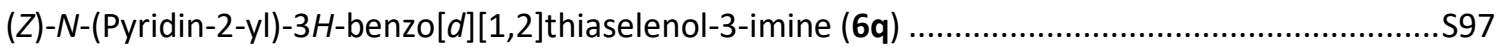

(Z)-5-Methyl-N-phenyl-3H-benzo[d][1,2]thiaselenol-3-imine (6r) ........................................S98

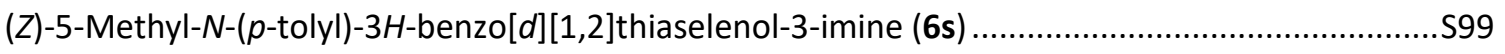

(Z)- $N$-(4-Chlorophenyl)-5-methyl-3H-benzo[d][1,2] thiaselenol-3-imine (6t) ............................S100

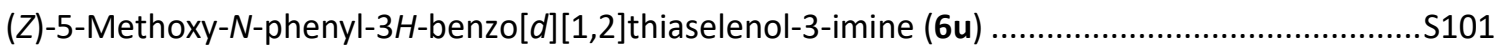

(Z)-5-Methoxy- $N$-(p-tolyl)-3H-benzo[d][1,2]thiaselenol-3-imine (6v) .......................................S102

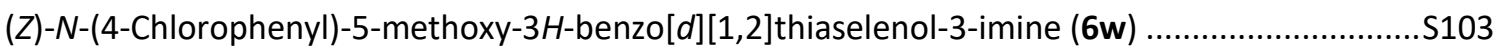

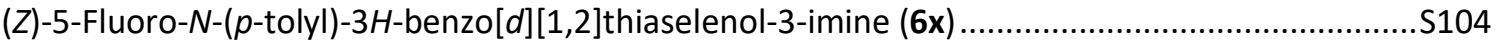

(Z)-N-(4-Chlorophenyl)-5-fluoro-3H-benzo[d][1,2]thiaselenol-3-imine (6y) ..............................S105

(Z)-5-Chloro- $N$-phenyl-3H-benzo[d] [1,2]thiaselenol-3-imine (6z) ......................................S106

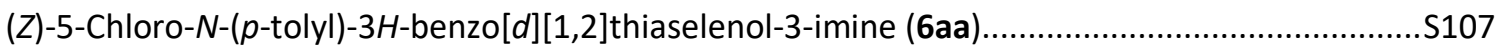

(Z)-5-Chloro- $N$-(4-chlorophenyl)-3H-benzo[d][1,2] thiaselenol-3-imine (6ab) .........................S108

(Z)-7-Methyl- $N$-(p-tolyl)-3H-benzo[d][1,2]thiaselenol-3-imine (6ac) .......................................S109

(Z)-N-(4-Chlorophenyl)-7-methyl-3H-benzo[d][1,2]thiaselenol-3-imine (6ad) .........................S110

$(6 Z, 12 Z)-N^{6}, N^{12}$-Diphenyl-6H,12H-dibenzo[ $\left.b, f\right][1,5]$ dithiocine-6,12-diimine (7a) ........................S111

$(6 Z, 12 Z)-N^{6}, N^{12}$-Di-p-tolyl-6H,12H-dibenzo[ $\left.b, f\right][1,5]$ dithiocine-6,12-diimine (7b) .......................S112

$(6 Z, 12 Z)-N^{6}, N^{12}$-Bis(4-methoxyphenyl)-6H,12H-dibenzo[ $\left.b, f\right][1,5]$ dithiocine-6,12-diimine (7c)......S113

$(6 Z, 12 Z)-N^{6}, N^{12}$-Bis(4-fluorophenyl)-6H,12H-dibenzo[b,f][1,5] dithiocine-6,12-diimine (7d).........S114

$(6 Z, 12 Z)-N^{6}, N^{12}$-Bis(4-chlorophenyl)-6H,12H-dibenzo[b,f][1,5]dithiocine-6,12-diimine (7e) .........S115

$(6 Z, 12 Z)-N^{6}, N^{12}$-Di- $m$-tolyl-6H,12H-dibenzo[ $\left.b, f\right][1,5]$ dithiocine-6,12-diimine (7f)......................S116

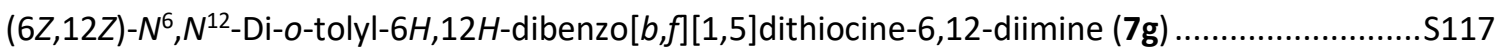

$(6 Z, 12 Z)-N^{6}, N^{12}$-Bis(3-chlorophenyl)-6H,12H-dibenzo[b,f][1,5]dithiocine-6,12-diimine (7h) .........S118 
$(6 Z, 12 Z)-N^{6}, N^{12}$-Bis(2-chlorophenyl)-6H,12H-dibenzo[ $\left.b, f\right][1,5]$ dithiocine-6,12-diimine (7i) S119

$(6 Z, 12 Z)-2,8$-Dimethyl- $N^{6}, N^{12}$-diphenyl-6H,12H-dibenzo[b,f][1,5]dithiocine-6,12-diimine (7j)......S120

$(6 Z, 12 Z)-2,8$-Dimethyl- $N^{6}, N^{12}$-di-p-tolyl-6H,12H-dibenzo[ $\left.b, f\right][1,5]$ dithiocine-6,12-diimine (7k) .....S121 $(6 Z, 12 Z)-N^{6}, N^{12}$-Bis(4-chlorophenyl)-2,8-dimethyl-6H,12H-dibenzo[ $\left.b, f\right][1,5]$ dithiocine-6,12-diimine (7I). S122

(6Z,12Z)-2,8-Dimethoxy- $N^{6}, N^{12}$-diphenyl-6H,12H-dibenzo[ $\left.b, f\right][1,5]$ dithiocine-6,12-diimine (7m)..S123 $(6 Z, 12 Z)$-2,8-Dimethoxy- $N^{6}, N^{12}$-di- $p$-tolyl-6H,12H-dibenzo[ $\left.b, f\right][1,5]$ dithiocine-6,12-diimine (7n) ..S124 $(6 Z, 12 Z)-N^{6}, N^{12}$-Bis(4-chlorophenyl)-2,8-dimethoxy-6H,12H-dibenzo[ $\left.b, f\right][1,5]$ dithiocine-6,12-diimine (7o) S125

$(6 Z, 12 Z)-2,8$-Dichloro- $N^{6}, N^{12}$-diphenyl-6H,12H-dibenzo[ $\left.b, f\right][1,5]$ dithiocine-6,12-diimine (7p)......S126 $(6 Z, 12 Z)-2,8$-Dichloro- $N^{6}, N^{12}$-di-p-tolyl-6H,12H-dibenzo[ $\left.b, f\right][1,5]$ dithiocine-6,12-diimine (7q) ......S127 (6Z,12Z)-2,8-Dichloro- $N^{6}, N^{12}$-bis(4-chlorophenyl)-6H,12H-dibenzo[ $\left.b, f\right][1,5]$ dithiocine-6,12-diimine (7r) S128

$(6 Z, 12 Z)-4,10$-Dimethyl- $N^{6}, N^{12}$-di-p-tolyl-6H,12H-dibenzo[ $\left.b, f\right][1,5]$ dithiocine-6,12-diimine (7s) ...S129 $(6 Z, 12 Z)-N^{6}, N^{12}$-Bis(4-chlorophenyl)-4,10-dimethyl-6H,12H-dibenzo[ $\left.b, f\right][1,5]$ dithiocine-6,12-diimine (7t) .S130 


\section{General information}

All reagents were purchased from commercial suppliers and used without treatment, unless otherwise indicated. Substrates 1 were prepared according to reported procedures. ${ }^{1}$ The products were purified by column chromatography on silica gel. ${ }^{1} \mathrm{H}$ NMR and ${ }^{13} \mathrm{C}$ NMR spectra were recorded on a Varian NMR spectrometer at $400 \mathrm{MHz}$ and $101 \mathrm{MHz}$, respectively. The NMR spectra for compounds 2-7 were recorded in $\mathrm{CDCl}_{3}$. Mass spectra were recorded on BRUKER AutoflexIII Smartbeam MS-spectrometer. High resolution mass spectra (HRMS) were recorded on Bruker microTOF using APCl ionization methods.

\section{Crystallography}

The structures of products $\mathbf{2 l}, \mathbf{6 u}$ and $\mathbf{7 n}$ were confirmed by the single crystal X-ray diffraction analysis. The X-ray diffraction data for the reported complexes was collected at 296(2) K on an Oxford Diffraction Gemini R Ultra diffractometer, using a $\omega$ scan technique with Mo-Ka radiation $(\lambda=$ $0.71073 \AA$ A). The structure was solved by Direct Method in SHELXS-97 and refined by full-matrix least-squares techniques using the SHELXL-97 program. $^{2}$ Non-hydrogen atoms were refined with anisotropic temperature parameters, and hydrogen atoms were refined as rigid groups. Basic information pertaining to crystal parameters and structure refinement is summarized in Tables S1, S2 and S3. 
Table S1. Crystal data and structure refinement of 2I (the crystal structure is with ellipsoids at 30\% probability level).

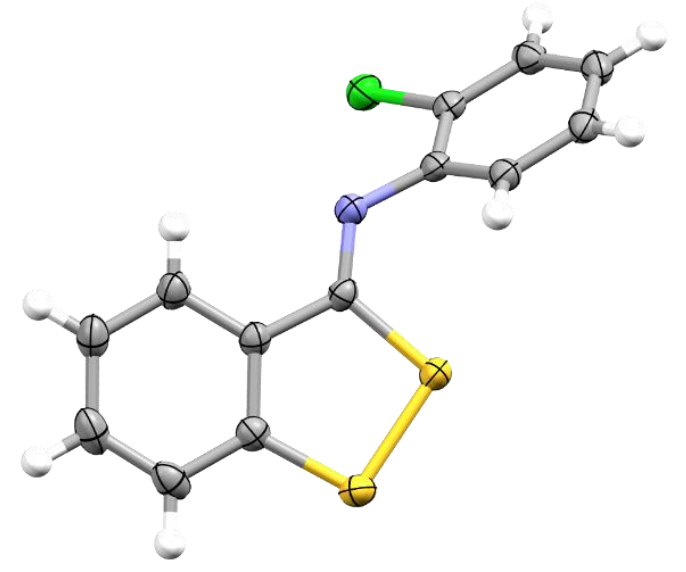

Empirical formula

Temperature

Wavelength

Crystal system, space group

Unit cell dimensions
$\mathrm{C}_{13} \mathrm{H}_{8} \mathrm{ClNS}_{2}$

296(2) K

$0.71073 \AA$

Monoclinic, P2(1)/c

$\mathrm{a}=14.1808$ (9) $\AA$

$\mathrm{b}=4.0804(3) \AA$

$c=20.7053(13) \AA$

alpha $=90.000 \mathrm{deg}$.

beta $=91.896(1) \mathrm{deg}$.

gamma $=90.000 \mathrm{deg}$.

Volume

1197.42(14) $\AA^{3}$

4

$1.541 \mathrm{Mg} / \mathrm{m}^{3}$

$0.640 \mathrm{~mm}^{-1}$

568

2.48 to $25.01 \mathrm{deg}$.

$-14<=\mathrm{h}<=16,-4<=\mathrm{k}<=4,-24<=\mathrm{k}<=24$

$6558 / 2105$ [R(int) $=0.0669]$

$99.5 \%$

None

Full-matrix least-squares on $\mathrm{F}^{2}$

2105 / 0 / 154

1.032

$\mathrm{R} 1=0.0320, \mathrm{wR} 2=0.0824$

$R 1=0.0338, w R 2=0.0842$

0.345 and -0.279 e. $A^{-3}$ 
Table S2. Crystal data and structure refinement of $\mathbf{6 u}$ (the crystal structure is with ellipsoids at $30 \%$ probability level).

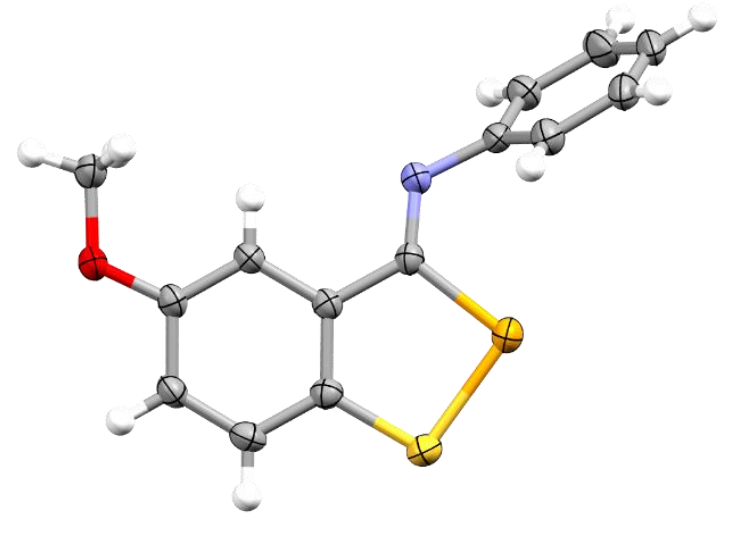

Empirical formula

Temperature

Wavelength

Crystal system, space group

Unit cell dimensions

Volume

Z

Calculated density

Absorption coefficient

$\mathrm{F}(000)$

Theta range for data collection

Limiting indices

Reflections collected / unique

Completeness to theta $=\mathbf{2 5 . 0 2}$

Absorption correction

Refinement method

Data / restraints / parameters

Goodness-of-fit on $\mathrm{F}^{\wedge} 2$

Final $R$ indices [ $>2$ sigma(I)]

$R$ indices (all data)

Largest diff. peak and hole
$\mathrm{C}_{14} \mathrm{H}_{11} \mathrm{NOSSe}$

296(2) K

$0.71073 \AA$

Triclinic, P1

$\mathrm{a}=4.545(8) \AA$

$\mathrm{b}=11.95(2) \AA$

$\mathrm{c}=12.09(2) \AA$

alpha $=88.22(2)$ deg.

beta $=85.63(2) \mathrm{deg}$.

gamma $=81.16(2) \mathrm{deg}$.

646.8(19) $\AA^{3}$

2

$1.644 \mathrm{Mg} / \mathrm{m}^{3}$

$3.049 \mathrm{~mm}^{-1}$

320

2.44 to $25.02 \mathrm{deg}$.

$5<=\mathrm{h}<=5,-8<=\mathrm{k}<=14,-14<=\mathrm{l}<=14$

$3761 / 2229[R($ int $)=0.0154]$

$97.0 \%$

None

Full-matrix least-squares on $\mathrm{F}^{2}$

2229 / 0 / 163

1.029

$R 1=0.0337, w R 2=0.0840$

$\mathrm{R} 1=0.0399, \mathrm{wR} 2=0.0870$

0.583 and -0.418 e. $A^{-3}$ 
Table S3. Crystal data and structure refinement of $\mathbf{7 n}$ (the crystal structure is with ellipsoids at $30 \%$ probability level).

Empirical formula

Temperature

Wavelength

Crystal system, space group

Unit cell dimensions
Volume

Z

Calculated density

Absorption coefficient

$\mathrm{F}(000)$

Theta range for data collection

Limiting indices

Reflections collected / unique

Completeness to theta $=\mathbf{2 5 . 0 2}$

Absorption correction

Refinement method

Data / restraints / parameters

Goodness-of-fit on $\mathrm{F}^{\wedge} 2$

Final $R$ indices [ $>2$ sigma $(I)]$

$R$ indices (all data)

Largest diff. peak and hole

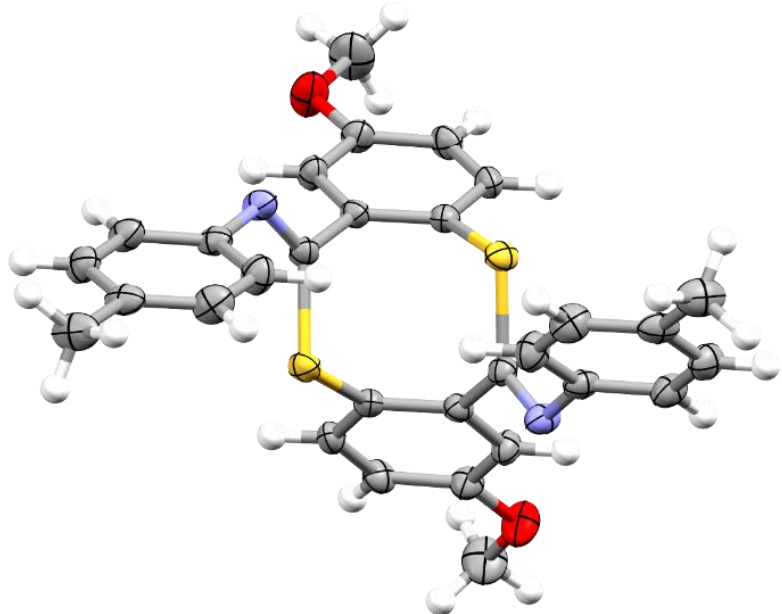

$\mathrm{C}_{30} \mathrm{H}_{26} \mathrm{~N}_{2} \mathrm{~S}_{2} \mathrm{O}_{2}$

296(2) K

$0.71073 \AA$

Monoclinic, Cc

$a=14.4163(12) \AA$

$\mathrm{b}=9.223483) \AA$

$c=20.234(2) \AA$

alpha $=90.000 \mathrm{deg}$.

beta $=104.539(1)$ deg.

gamma $=90.000 \mathrm{deg}$.

2604.3(4) $\AA^{3}$

4

$1.302 \mathrm{Mg} / \mathrm{m}^{3}$

$0.235 \mathrm{~mm}^{-1}$

1072

2.65 to 25.02 deg.

$-17<=h<=17,-8<=k<=10,-24<=k<=22$

$7541 / 2284[\mathrm{R}$ (int) $=0.0760]$

$99.9 \%$

None

Full-matrix least-squares on $\mathrm{F}^{2}$

2284 / 0 / 165

1.036

$R 1=0.0396, w R 2=0.1025$

$\mathrm{R} 1=0.0480, w R 2=0.1079$

0.251 and -0.255 e. $A^{-3}$ 


\section{Synthesis and analytical data}

\section{General procedure for the synthesis of 2 (using $2 a$ as an example)}<smiles>S=C(Nc1ccccc1)c1ccccc1Br</smiles>

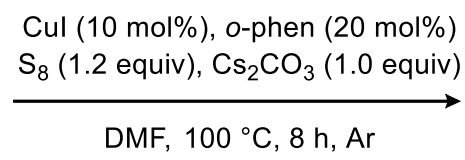<smiles>c1ccc(/N=C2\Sc3ccccc32)cc1</smiles>

2-bromo- $N$-phenylbenzothioamide 1a (146 mg, $0.5 \mathrm{mmol}$ ), $\mathrm{S}_{8}(154 \mathrm{mg}, 0.6 \mathrm{mmol}$ ), Cul (10 mg, 0.05 mmol), o-phen (18 mg, $0.1 \mathrm{mmol}), \mathrm{Cs}_{2} \mathrm{CO}_{3}(163 \mathrm{mg}, 0.5 \mathrm{mmol})$ were added to a dry reaction flask connected to a high-vacuum valve. The flask was then evacuated and backfilled three times with $\mathrm{Ar}$ after which DMF $(5.0 \mathrm{~mL})$ was added to the mixture. The reaction mixture was stirred at $100{ }^{\circ} \mathrm{C}$ in an oil bath for $8 \mathrm{~h}$ under an atmosphere of Ar. Then, the mixture was poured into water $(50 \mathrm{~mL})$ and the formed yellow precipitate was collected by filtration. The crude product was purified by chromatography (silica gel; petroleum ether/ethyl acetate 10:1) to give $\mathbf{2 a}$ as a yellow solid (104 mg, $85 \%)$.

\section{$2.0 \mathrm{mmol}-\mathrm{Scale}$ reaction}<smiles>S=C(Nc1ccccc1)c1ccccc1Br</smiles>

$$
\begin{aligned}
& \text { Cul (10 mol\%), o-phen (20 mol\%) } \\
& \frac{\mathrm{S}_{8}\left(1.2 \text { equiv), } \mathrm{Cs}_{2} \mathrm{CO}_{3}(1.0 \text { equiv })\right.}{\mathrm{DMF}, 100{ }^{\circ} \mathrm{C}, 8 \mathrm{~h}, \mathrm{Ar}}
\end{aligned}
$$

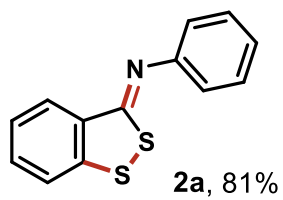

2-bromo- $N$-phenylbenzothioamide 1a (584 mg, $2.0 \mathrm{mmol}$ ), $\mathrm{S}_{8}(616 \mathrm{mg}, 2.4 \mathrm{mmol}$ ), Cul (40 mg, 0.20 mmol), o-phen (72 mg, $0.4 \mathrm{mmol}), \mathrm{Cs}_{2} \mathrm{CO}_{3}(652 \mathrm{mg}, 2.0 \mathrm{mmol})$ were added to a dry reaction flask connected to a high-vacuum valve. The flask was then evacuated and backfilled three times with $\mathrm{Ar}$ after which DMF $(20 \mathrm{~mL})$ was added to the mixture. The reaction mixture was stirred at $100{ }^{\circ} \mathrm{C}$ in an oil bath for $8 \mathrm{~h}$ under an atmosphere of Ar. Then, the mixture was poured into water $(200 \mathrm{~mL})$ and the formed yellow precipitate was collected by filtration. The crude product was purified by 
chromatography (silica gel; petroleum ether/ethyl acetate 10:1) to give $\mathbf{2 a}$ as a yellow solid (395 mg, $81 \%)$.

\section{General procedure for the synthesis of 3 (using 3a as an example)}

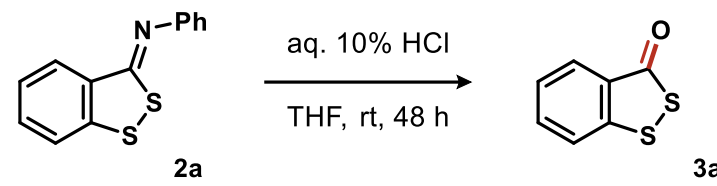

To a $10 \mathrm{~mL}$ round-bottom flask, (Z)- $N$-phenyl-3H-benzo[c][1,2]dithiol-3-imine $2 \mathrm{a}$ (122 mg, $0.5 \mathrm{mmol}$ ), aq. $\mathrm{HCl}(10 \%, 1 \mathrm{~mL})$ and THF $(1 \mathrm{~mL})$ were added. The reaction mixture was stirred at room temperature until substrate $\mathbf{2 a}$ was consumed as indicated by TLC (about $48 \mathrm{~h}$ ). The resulting mixture was concentrated and the residue was taken up in $\mathrm{CH}_{2} \mathrm{Cl}_{2}$. The organic layer was washed with brine, dried over $\mathrm{MgSO}_{4}$ and concentrated. Purification of the crude product by column chromatography (silica gel; petroleum ether/ethyl acetate 20:1) afforded 3a as a white solid (68 mg, $81 \%)$.

\section{Synthesis of dioxide 4}<smiles></smiles>

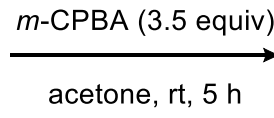<smiles>O=C1SS(=O)(=O)c2ccccc21</smiles>

To a $10 \mathrm{~mL}$ round-bottom flask, $3 \mathrm{H}$-benzo[c][1,2]dithiol-3-one $3 \mathrm{3a}(84 \mathrm{mg}, 0.5 \mathrm{mmol}$ ), $\mathrm{m}$-CPBA (301 $\mathrm{mg}, 1.75 \mathrm{mmol}$ ) and acetone $(2 \mathrm{~mL})$ were added. The reaction mixture was stirred at room temperture until substrate $3 a$ was consumed as indicated by TLC (about $5 \mathrm{~h}$ ). The resulting mixture was concentrated and the residue was taken up in $\mathrm{CH}_{2} \mathrm{Cl}_{2}$. The organic layer was washed with brine, dried over $\mathrm{MgSO}_{4}$ and concentrated. Purification of the crude product by column chromatography 
(silica gel; petroleum ether/ethyl acetate $10: 1$ ) afforded $\mathbf{4 a}$ as a white solid (71 mg, $71 \%$ ).

\section{Synthesis of oxide 5}<smiles></smiles>

3a

$$
\underset{\text { TFA, } 40^{\circ} \mathrm{C}, 2 \mathrm{~h}}{\stackrel{\text { aq. } 30 \% \mathrm{H}_{2} \mathrm{O}_{2}}{\longrightarrow}}
$$<smiles>O=C1OS(=O)c2ccccc21</smiles>

To a $10 \mathrm{~mL}$ round-bottom flask, $3 \mathrm{H}$-benzo[c][1,2]dithiol-3-one $3 a(84 \mathrm{mg}, 0.5 \mathrm{mmol}), 30 \% \mathrm{H}_{2} \mathrm{O}_{2}(1$ $\mathrm{mL})$, TFA (1 mL) were added. The reaction mixture was stirred at $40{ }^{\circ} \mathrm{C}$ in an oil bath until substrate 3a was consumed as indicated by TLC (about $2 \mathrm{~h}$ ). The resulting mixture was concentrated and the residue was taken up in $\mathrm{CH}_{2} \mathrm{Cl}_{2}$. The organic layer was washed with brine, dried over $\mathrm{MgSO}_{4}$ and concentrated. Purification of the crude product by column chromatography (silica gel; petroleum ether/ethyl acetate 15:1) afforded 5 as a white solid (30 mg, 33\%).

\section{General procedure for the synthesis of 6 (using $6 a$ as an example)}<smiles>S=C(Nc1ccccc1)c1ccccc1Br</smiles>

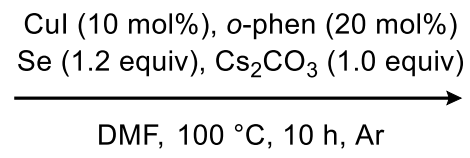<smiles>c1ccc(/N=C2\Sc3ccccc32)cc1</smiles>

The procedure is the same as that of $2 a$ except that $S_{8}$ was replaced with $S_{e}(48 \mathrm{mg}, 0.6 \mathrm{mmol}$ ), the reaction time was extended to $10 \mathrm{~h}$ and the crude product was purified using petroleum ether as eluent. This afforded $6 \mathrm{a}$ as a yellow solid (101 $\mathrm{mg}, 69 \%)$. 
General procedure for the synthesis of 7 (using $7 a$ as an example)<smiles>S=C(Nc1ccccc1)c1ccccc1Br</smiles>
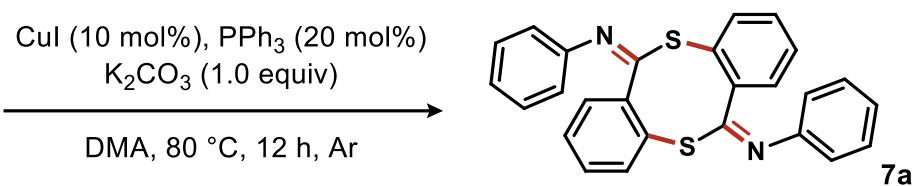

2-bromo- $N$-phenylbenzothioamide 1a (146 mg, $0.5 \mathrm{mmol}$ ), Cul (10 mg, $0.05 \mathrm{mmol}$ ), $\mathrm{PPh}_{3}$ (26 mg, 0.1 mmol), $\mathrm{K}_{2} \mathrm{CO}_{3}(69 \mathrm{mg}, 0.5 \mathrm{mmol}$ ) were added to a dry reaction flask connected to a high-vacuum valve. The flask was then evacuated and backfilled three times with Ar after which DMA $(5.0 \mathrm{~mL})$ was added to the mixture. The reaction mixture was stirred at $80{ }^{\circ} \mathrm{C}$ in an oil bath for $12 \mathrm{~h}$ under an atmosphere of Ar. The mixture was poured into water $(50 \mathrm{~mL})$ and the formed yellow precipitate was collected by filtration. The crude product was purified by chromatography (silica; petroleum ether/ethyl acetate $8: 1$ ) to give $7 \mathrm{a}$ as a white solid (190 $\mathrm{mg}, 79 \%)$.

(Z)-N-Phenyl-3H-benzo[c][1,2]dithiol-3-imine (2a)<smiles>c1ccc(/N=c2\ssc3ccccc23)cc1</smiles>

Yield: 85\% (104 mg). Yellow solid; mp 67-68 ${ }^{\circ} \mathrm{C} ;{ }^{1} \mathrm{H}$ NMR $\left(400 \mathrm{MHz}, \mathrm{CDCl}_{3}\right) \delta 8.15$ $(\mathrm{d}, J=8.0 \mathrm{~Hz}, 1 \mathrm{H}), 7.46-7.38(\mathrm{~m}, 4 \mathrm{H}), 7.31-7.28(\mathrm{~m}, 1 \mathrm{H}), 7.19-7.13(\mathrm{~m}, 3 \mathrm{H}) ;{ }^{13} \mathrm{C}$ NMR $\left(101 \mathrm{MHz}, \mathrm{CDCl}_{3}\right) \delta 166.3,151.5,145.3,132.6,131.8,129.7,126.8,125.4,125.1,123.3,119.8 ;$ $\operatorname{IR}(\mathrm{KBr})$ v 3058, 1615, 1592, 1582, 1483, 1441, 1261, 1245, 1154, 1096, 1073, 1064, 1024, 923, 803, 757, 721, 691, $663 \mathrm{~cm}^{-1}$; HRMS (APCl) $\mathrm{m} / z$ calculated for $\mathrm{C}_{13} \mathrm{H}_{10} \mathrm{NS}_{2}[\mathrm{M}+\mathrm{H}]^{+}: 244.0249$, found 244.0265. 
(Z)-N-(p-Tolyl)-3H-benzo[c][1,2]dithiol-3-imine (2b)

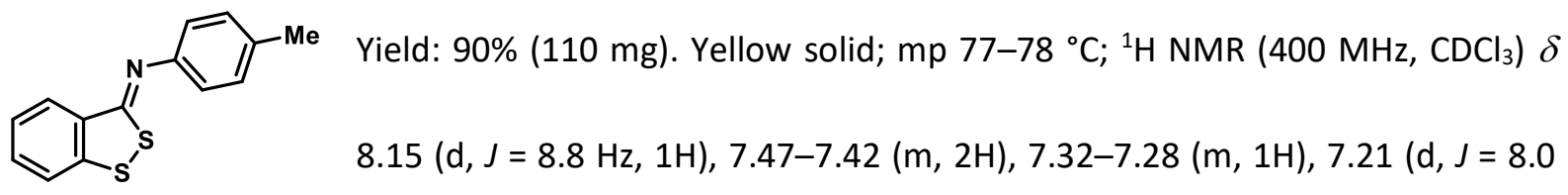

$\mathrm{Hz}, 2 \mathrm{H}), 7.05(\mathrm{~d}, J=7.2 \mathrm{~Hz}, 2 \mathrm{H}), 2.35(\mathrm{~s}, 3 \mathrm{H}) ;{ }^{13} \mathrm{C} \mathrm{NMR}\left(101 \mathrm{MHz}, \mathrm{CDCl}_{3}\right) \delta 164.8,147.9,144.2,133.8$, 131.7, 130.6, 129.2, 125.7, 124.4, 122.3, 118.8, 20.0; IR (KBr) v 3057, 2914, 2851, 1618, 1599, 1505, $1441,1278,1244,1190,1159,1069,951,913,816,757,720,708,657,607 \mathrm{~cm}^{-1} ;$ HRMS (APCI) m/z calculated for $\mathrm{C}_{14} \mathrm{H}_{12} \mathrm{NS}_{2}[\mathrm{M}+\mathrm{H}]^{+}: 258.0406$, found 258.0411 .

(Z)-N-(4-Isopropylphenyl)-3H-benzo[c][1,2]dithiol-3-imine (2c)

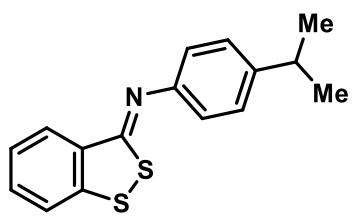

Yield: $86 \%$ (123 mg). Yellow solid; $\mathrm{mp} 74-75{ }^{\circ} \mathrm{C} ;{ }^{1} \mathrm{H} \mathrm{NMR}\left(400 \mathrm{MHz}, \mathrm{CDCl}_{3}\right) \delta$ 8.19-8.16 (m, 1H), 7.52-7.47 (m, 2H), 7.37-7.33 (m, 1H), 7.30-7.26 (m, 2H), 7.12-7.08 (m, 2H), 2.99-2.88 (m, 1H), $1.29(\mathrm{~s}, 3 \mathrm{H}), 1.27(\mathrm{~s}, 3 \mathrm{H}) ;{ }^{13} \mathrm{C} N M R$ $\left(101 \mathrm{MHz}, \mathrm{CDCl}_{3}\right) \delta 165.7,149.2,145.9,145.2,132.9,131.7,127.6,126.9,125.5,123.3,119.8,33.7$, 24.1; IR (KBr) v 3052, 2952, 2916, 2859, 1614, 1598, 1500, 1456, 1276, 1241, 1184, 1197, 1066, 1047, 923, 835, 755, 722, 694, $655 \mathrm{~cm}^{-1}$; HRMS (APCl) $\mathrm{m} / z$ calculated for $\mathrm{C}_{16} \mathrm{H}_{16} \mathrm{NS}_{2}[\mathrm{M}+\mathrm{H}]^{+}: 286.0719$, found 286.0730 .

(Z)-N-(4-Methoxyphenyl)-3H-benzo[c][1,2]dithiol-3-imine (2d)

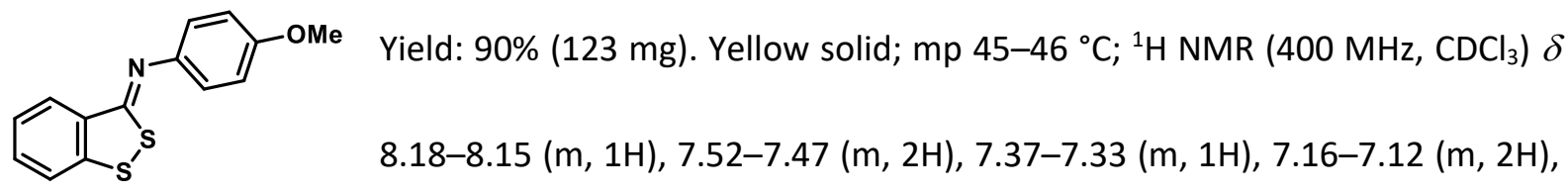
6.99-6.95 (m, 2H), $3.84(\mathrm{~s}, 3 \mathrm{H}) ;{ }^{13} \mathrm{C}$ NMR $\left(101 \mathrm{MHz}, \mathrm{CDCl}_{3}\right) \delta 165.5,157.2,145.1,144.7,132.9,131.7$, $126.8,125.5,123.4,121.4,114.8,55.5 ; \operatorname{IR~}(\mathrm{KBr})$ v 3063, 2960, 2835, 1607, 1597, 1501, 1462, 1440, 
$1287,1274,1238,1180,1066,1026,933,912,839,760,704,652 \mathrm{~cm}^{-1}$; HRMS (APCI) $\mathrm{m} / \mathrm{z}$ calculated for $\mathrm{C}_{14} \mathrm{H}_{12} \mathrm{NOS}_{2}[\mathrm{M}+\mathrm{H}]^{+}: 274.0355$, found 274.0381 .

(Z)-N-(4-Fluorophenyl)-3H-benzo[c][1,2]dithiol-3-imine (2e)

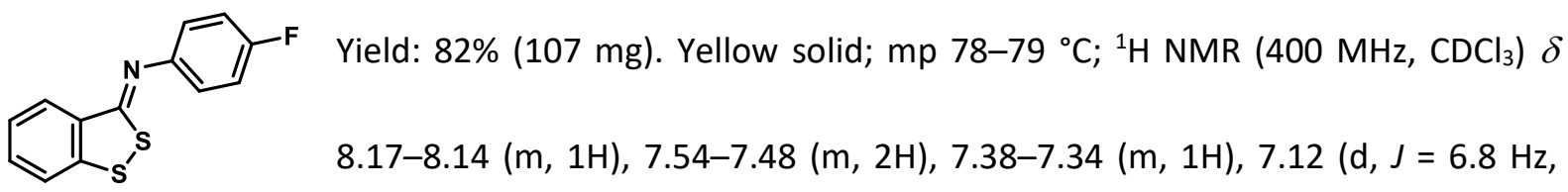
$4 \mathrm{H}) ;{ }^{13} \mathrm{C}$ NMR $\left(101 \mathrm{MHz}, \mathrm{CDCl}_{3}\right) \delta 167.0\left(\mathrm{~d}, J_{\mathrm{C}-\mathrm{F}}=2.3 \mathrm{~Hz}\right), 160.2\left(\mathrm{~d}, J_{\mathrm{C}-\mathrm{F}}=242.7 \mathrm{~Hz}\right), 147.6\left(\mathrm{~d}, J_{\mathrm{C}-\mathrm{F}}=3.0\right.$ $\mathrm{Hz}), 145.4,132.6,132.0,126.8,125.6,123.4,121.5\left(\mathrm{~d}, J_{\mathrm{C}-\mathrm{F}}=8.2 \mathrm{~Hz}\right), 116.5\left(\mathrm{~d}, J_{\mathrm{C}-\mathrm{F}}=22.4 \mathrm{~Hz}\right) ; \mathrm{IR}(\mathrm{KBr})$ $v 3067,3058,1592,1583,1501,1461,1440,1308,1215,1177,1151,1095,1069,916,834,775,750$, $720,707,657 \mathrm{~cm}^{-1}$; HRMS (APCl) $\mathrm{m} / z$ calculated for $\mathrm{C}_{13} \mathrm{H}_{9} \mathrm{FNS}_{2}[\mathrm{M}+\mathrm{H}]^{+}: 262.0155$, found 262.0164 .

\section{(Z)-N-(4-Chlorophenyl)-3H-benzo[c][1,2]dithiol-3-imine (2f)}

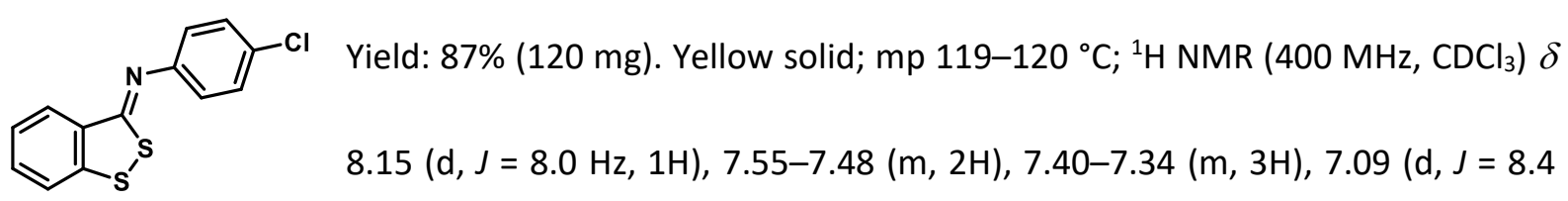

$\mathrm{Hz}, 2 \mathrm{H}) ;{ }^{13} \mathrm{C}$ NMR $\left(101 \mathrm{MHz}, \mathrm{CDCl}_{3}\right) \delta 166.1,148.9,144.4,131.5,131.0,129.3,128.8,125.8,124.6$, $122.3,120.4 ; \mathrm{IR}(\mathrm{KBr}) v 3060,1610,1581,1484,1442,1398,1278,1243,1189,1160,1123,1088$ 1071, 1005, 915, 824, 760, 721, $629 \mathrm{~cm}^{-1}$; HRMS (APCl) $\mathrm{m} / \mathrm{z}$ calculated for $\mathrm{C}_{13} \mathrm{H}_{9} \mathrm{ClNS}_{2}[\mathrm{M}+\mathrm{H}]^{+}$: 277.9859 , found 277.9894 . 
(Z)-N-(m-Tolyl)-3H-benzo[c][1,2]dithiol-3-imine (2g)

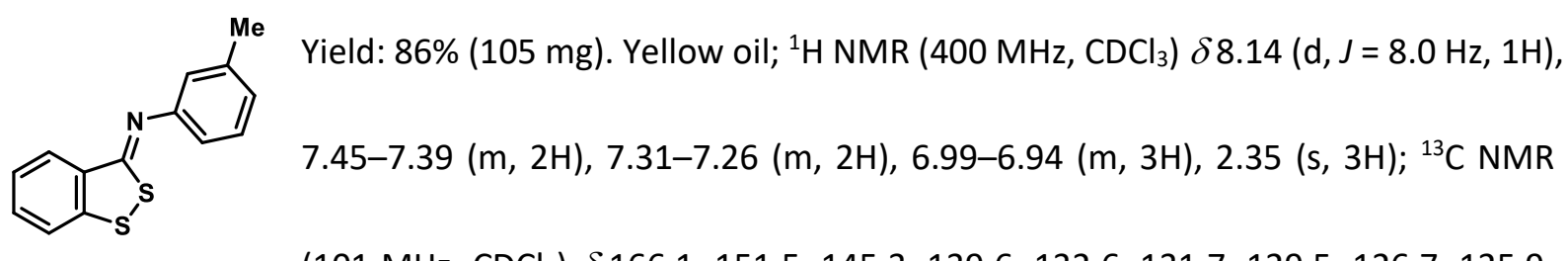
$\left(101 \mathrm{MHz}, \mathrm{CDCl}_{3}\right) \delta 166.1,151.5,145.3,139.6,132.6,131.7,129.5,126.7,125.9$, $125.4,123.3,120.5,116.6,21.5 ; \operatorname{IR}(\mathrm{KBr})$ v 3050, 3022, 2963, 1607, 1581, 1481, 1439, 1303, 1262, 1243, 1161, 1107, 1061, 1062, 1020, 926, 766, 755, 718, 697, $661 \mathrm{~cm}^{-1}$; HRMS (APCI) $\mathrm{m} / \mathrm{z}$ calculated for $\mathrm{C}_{14} \mathrm{H}_{12} \mathrm{NS}_{2}[\mathrm{M}+\mathrm{H}]^{+}:$258.0406, found 258.0408 .

(Z)-N-(3-Chlorophenyl)-3H-benzo[c][1,2]dithiol-3-imine (2h)

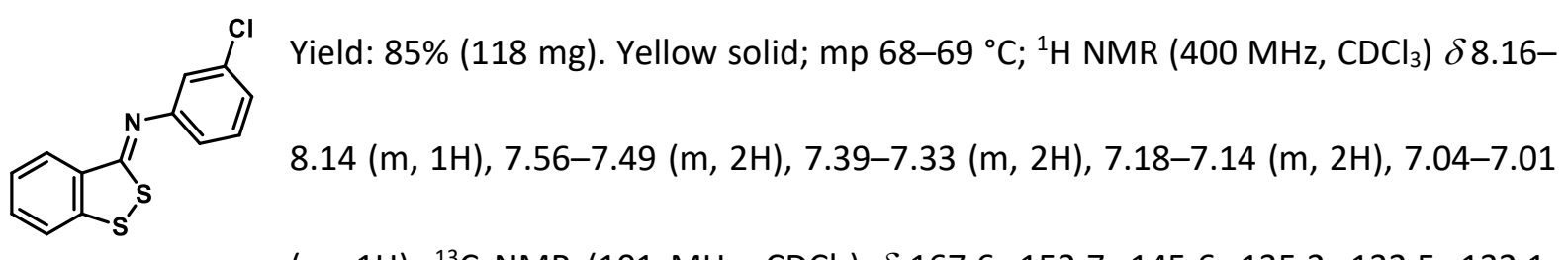
$(\mathrm{m}, 1 \mathrm{H}) ;{ }^{13} \mathrm{C}$ NMR $\left(101 \mathrm{MHz}, \mathrm{CDCl}_{3}\right) \delta 167.6,152.7,145.6,135.2,132.5,132.1$ $130.8,126.9,125.6,125.1,123.4,120.3,118.1$; IR (KBr) v 3054, 1600, 1576, 1559, 1472, 1439, 1385, 1261, 1180, 1066, 921, 884, 861, 785, 793, 735, 720, 688, $657 \mathrm{~cm}^{-1}$; HRMS (APCI) $\mathrm{m} / \mathrm{z}$ calculated for $\mathrm{C}_{13} \mathrm{H}_{9} \mathrm{CINS}_{2}[\mathrm{M}+\mathrm{H}]^{+}:$277.9859, found 277.9858.

(Z)-N-(3-Nitrophenyl)-3H-benzo[c][1,2]dithiol-3-imine (2i)

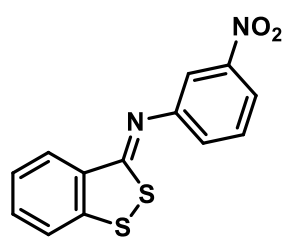

Yield: 85\% (123 mg). Yellow solid; mp $144-145{ }^{\circ} \mathrm{C} ;{ }^{1} \mathrm{H} \mathrm{NMR}\left(400 \mathrm{MHz}, \mathrm{CDCl}_{3}\right) \delta$ $8.18(\mathrm{~d}, J=8.0 \mathrm{~Hz}, 1 \mathrm{H}), 8.06-8.02(\mathrm{~m}, 2 \mathrm{H}), 7.61-7.53(\mathrm{~m}, 3 \mathrm{H}), 7.46(\mathrm{~d}, J=8.0 \mathrm{~Hz}$,

1H), $7.41(\mathrm{t}, J=7.2 \mathrm{~Hz}, 1 \mathrm{H}) ;{ }^{13} \mathrm{C}$ NMR $\left(101 \mathrm{MHz}, \mathrm{CDCl}_{3}\right) \delta 168.8,152.4,149.3$, $145.8,132.4,132.4,130.6,126.9,126.6,125.8,123.4,119.7,115.4 ; \mathrm{IR}(\mathrm{KBr})$ v 3067, 1592, 1581, 1524, 1439, 1349, 1313, 1268, 1182, 1082, 1065, 913, 820, 805, 755, 739, 721, $682 \mathrm{~cm}^{-1}$; HRMS (APCI) 
$m / z$ calculated for $\mathrm{C}_{13} \mathrm{H}_{9} \mathrm{~N}_{2} \mathrm{O}_{2} \mathrm{~S}_{2}[\mathrm{M}+\mathrm{H}]^{+}:$289.0101, found 289.0110 .

(Z)-N-(o-Tolyl)-3H-benzo[c][1,2]dithiol-3-imine (2j)

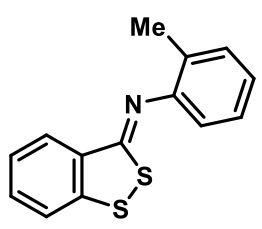

Yield: 88\% (107 mg). Yellow solid; mp 41-42 ${ }^{\circ} \mathrm{C} ;{ }^{1} \mathrm{H} \mathrm{NMR}\left(400 \mathrm{MHz}, \mathrm{CDCl}_{3}\right) \delta 8.21$

$(\mathrm{d}, J=8.0 \mathrm{~Hz}, 1 \mathrm{H}), 7.55-7.48(\mathrm{~m}, 2 \mathrm{H}), 7.39-7.35(\mathrm{~m}, 1 \mathrm{H}), 7.29(\mathrm{~d}, J=7.6 \mathrm{~Hz}, 1 \mathrm{H})$,

$7.25-7.22(\mathrm{~m}, 1 \mathrm{H}), 7.11(\mathrm{t}, J=7.6 \mathrm{~Hz}, 1 \mathrm{H}), 7.03(\mathrm{~d}, J=7.6 \mathrm{~Hz}, 1 \mathrm{H}), 2.23(\mathrm{~s}, 3 \mathrm{H}) ;{ }^{13} \mathrm{C}$

NMR $\left(101 \mathrm{MHz}, \mathrm{CDCl}_{3}\right) \delta 166.2,150.6,145.6,132.3,131.8,131.1,129.1,127.1,126.9,125.5,125.2$

123.5, 118.1, 17.6; IR (KBr) v 3066, 3016, 2972, 2919, 1626, 1614, 1573, 1482, 1441, 1384, 1372,

$1276,1241,1120,930,915,844,752,718,661 \mathrm{~cm}^{-1}$; HRMS (APCl) $\mathrm{m} / z$ calculated for $\mathrm{C}_{14} \mathrm{H}_{12} \mathrm{NS}_{2}[\mathrm{M}+$ $H]^{+}:$258.0406, found 258.0403 .

(Z)-N-(2-Ethoxyphenyl)-3H-benzo[c][1,2]dithiol-3-imine (2k)<smiles>CCOc1ccccc1/N=c1\ssc2ccccc12</smiles>

Yield: 83\% (119 mg). Yellow solid; $\mathrm{mp} 60-61{ }^{\circ} \mathrm{C} ;{ }^{1} \mathrm{H} \mathrm{NMR}\left(400 \mathrm{MHz}, \mathrm{CDCl}_{3}\right) \delta 8.25-$ $8.22(m, 1 H), 7.54-7.47(m, 2 H), 7.38-7.34(m, 1 H), 7.17-7.13(m, 1 H), 7.08-7.06$ (m, 1H), 7.01-6.97 (m, 2H), $4.09(\mathrm{q}, J=7.2 \mathrm{~Hz}, 2 \mathrm{H}), 1.36(\mathrm{t}, J=6.8 \mathrm{~Hz}, 3 \mathrm{H}) ;{ }^{13} \mathrm{C} \mathrm{NMR}$

$\left(101 \mathrm{MHz}, \mathrm{CDCl}_{3}\right) \delta 167.4,149.4,145.6,141.1,132.5,131.7,127.0,126.0,125.4,123.4,121.4,120.4$, 114.0, 64.5, 14.9; IR (KBr) v 3058, 2978, 2935, 2870, 1598, 1578, 1493, 1474, 1442, 1394, 1251, 1229 , $1114,1067,1042,928,745,721,667 \mathrm{~cm}^{-1}$; HRMS (APCI) $\mathrm{m} / \mathrm{z}$ calculated for $\mathrm{C}_{15} \mathrm{H}_{14} \mathrm{NOS}_{2}[\mathrm{M}+\mathrm{H}]^{+}$: 288.0511 , found 288.0523 . 
(Z)-N-(2-Chlorophenyl)-3H-benzo[c][1,2]dithiol-3-imine (2I)<smiles>Clc1ccccc1/N=c1\ssc2ccccc12</smiles>

Yield: 86\% (119 mg). Yellow solid; $\mathrm{mp} 75-76{ }^{\circ} \mathrm{C} ;{ }^{1} \mathrm{H} \mathrm{NMR}\left(400 \mathrm{MHz}, \mathrm{CDCl}_{3}\right) \delta 8.24$ $(\mathrm{d}, J=8.0 \mathrm{~Hz}, 1 \mathrm{H}), 7.57-7.48(\mathrm{~m}, 3 \mathrm{H}), 7.41-7.37(\mathrm{~m}, 1 \mathrm{H}), 7.31(\mathrm{t}, J=8.0 \mathrm{~Hz}, 1 \mathrm{H})$, 7.15-7.11 (m, 2H); ${ }^{13} \mathrm{C}$ NMR $\left(101 \mathrm{MHz}, \mathrm{CDCl}_{3}\right) \delta 168.6,148.6,145.9,132.2,132.1$, $130.6,128.0,127.0,125.9,125.7,125.6,123.4,120.3 ; \mathrm{IR}(\mathrm{KBr})$ v 3050, 1616, 1578, 1471, 1438, 1277, 1259, 1243, 1196, 1181, 1159, 1055, 1035, 913, 837,758, 750, 719, 668, $655 \mathrm{~cm}^{-1}$; HRMS (APCI) m/z calculated for $\mathrm{C}_{13} \mathrm{H}_{9} \mathrm{CINS}_{2}[\mathrm{M}+\mathrm{H}]^{+}:$277.9859, found 277.9895 .

(Z)-N-(2,4-Dichlorophenyl)-3H-benzo[c][1,2]dithiol-3-imine (2m)<smiles>Clc1ccc(/N=c2\ssc3ccccc23)c(Cl)c1</smiles>

Yield: $89 \%$ (139 mg). Yellow solid; mp $126-127^{\circ} \mathrm{C} ;{ }^{1} \mathrm{H}$ NMR (400 MHz, $\left.\mathrm{CDCl}_{3}\right) \delta$ $8.22(\mathrm{~d}, J=8.0 \mathrm{~Hz}, 1 \mathrm{H}), 7.59-7.50(\mathrm{~m}, 3 \mathrm{H}), 7.42-7.38(\mathrm{~m}, 1 \mathrm{H}), 7.30-7.26(\mathrm{~m}$, $1 \mathrm{H}), 7.08(\mathrm{~d}, J=8.4 \mathrm{~Hz}, 1 \mathrm{H}) ;{ }^{13} \mathrm{C} N M R\left(101 \mathrm{MHz}, \mathrm{CDCl}_{3}\right) \delta 169.3,147.3,146.1$, $132.4,132.2,130.5,128.3,127.1,126.7,125.8,123.5,121.3 ; \mathrm{IR}(\mathrm{KBr})$ v 3052, 1605, 1575, 1471, 1458, $1385,1257,1100,1192,1177,1161,1069,1058,913,866,812,757,721,707,664 \mathrm{~cm}^{-1}$; HRMS (APCI) $m / z$ calculated for $\mathrm{C}_{13} \mathrm{H}_{8} \mathrm{Cl}_{2} \mathrm{NS}_{2}[\mathrm{M}+\mathrm{H}]^{+}: 311.9470$, found 311.9486 .

(Z)-N-(3,4-Dimethoxyphenyl)-3H-benzo[c][1,2]dithiol-3-imine (2n)

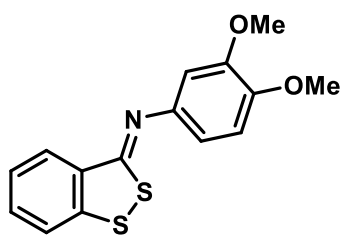

Yield: 89\% (128 mg). Yellow solid; mp $129-130{ }^{\circ} \mathrm{C} ;{ }^{1} \mathrm{H} \mathrm{NMR}\left(400 \mathrm{MHz}, \mathrm{CDCl}_{3}\right)$ $\delta 8.17(\mathrm{~d}, J=8.0 \mathrm{~Hz}, 1 \mathrm{H}), 7.53-7.48(\mathrm{~m}, 2 \mathrm{H}), 7.37-7.33(\mathrm{~m}, 1 \mathrm{H}), 7.92(\mathrm{~d}, J=$ $8.0 \mathrm{~Hz}, 1 \mathrm{H}), 6.08-6.78(\mathrm{~m}, 2 \mathrm{H}), 3.92(\mathrm{~s}, 3 \mathrm{H}), 3.90(\mathrm{~s}, 3 \mathrm{H}) ;{ }^{13} \mathrm{C}$ NMR $(101 \mathrm{MHz}$, $\left.\mathrm{CDCl}_{3}\right) \delta 165.8,149.8,146.6,145.1,145.0,132.9,131.8,126.8,125.5,123.4,111.7,111.1,104.8$ 56.1, 55.9; IR (KBr) v 3063, 3008, 2954, 2934, 2834, 1608, 1506, 1462, 1326, 1258, 1231, 1186, 1126, 
1069, 1020, 949, 909, 846, 765, $664 \mathrm{~cm}^{-1}$; HRMS (APCI) $\mathrm{m} / z$ calculated for $\mathrm{C}_{15} \mathrm{H}_{14} \mathrm{NO}_{2} \mathrm{~S}_{2}[\mathrm{M}+\mathrm{H}]^{+}$: 304.0460 , found 304.0485 .

\section{(Z)-N-(Pyridin-2-yl)-3H-benzo[c][1,2]dithiol-3-imine (2o)}

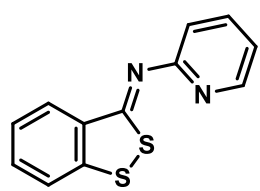

Yield: $81 \%$ (99 mg). Yellow solid; $\mathrm{mp} 204-205{ }^{\circ} \mathrm{C} ;{ }^{1} \mathrm{H}$ NMR $\left(400 \mathrm{MHz}, \mathrm{CDCl}_{3}\right) \delta$ 8.61-8.59 (m, 1H), $8.56(\mathrm{~d}, J=8.0 \mathrm{~Hz}, 1 \mathrm{H}), 7.87-7.83(\mathrm{~m}, 1 \mathrm{H}), 7.68(\mathrm{~d}, J=8.0 \mathrm{~Hz}$, $1 \mathrm{H}), 7.60(\mathrm{~d}, J=8.0 \mathrm{~Hz}, 1 \mathrm{H}), 7.57-7.52(\mathrm{~m}, 1 \mathrm{H}), 7.41-7.37(\mathrm{~m}, 1 \mathrm{H}), 7.19-7.16(\mathrm{~m}, 1 \mathrm{H}) ;{ }^{13} \mathrm{C}$ NMR $(101$ $\left.\mathrm{MHz}, \mathrm{CDCl}_{3}\right) \delta 170.1,156.5,146.8,142.5,138.0,134.8,131.2,127.2,124.5,123.8,121.2,119.5 ; \mathrm{IR}$ $(\mathrm{KBr})$ v 3062, 1606, 1598, 1588, 1560, 1508, 1464, 1433, 1421, 1316, 1261, 1097, 1024, 946, 864, 801, $747,738,663 \mathrm{~cm}^{-1}$; HRMS (APCl) $\mathrm{m} / z$ calculated for $\mathrm{C}_{12} \mathrm{H}_{9} \mathrm{~N}_{2} \mathrm{~S}_{2}[\mathrm{M}+\mathrm{H}]^{+}: 245.0202$, found 245.0195 .

\section{(Z)-N-(9H-Fluoren-2-yl)-3H-benzo[c][1,2]dithiol-3-imine (2p)}

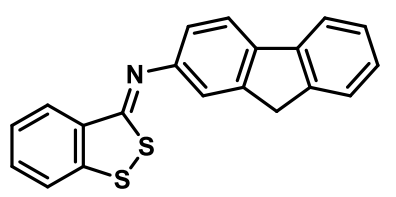

Yield: 90\% (149 mg). Yellow solid; mp $147-148{ }^{\circ} \mathrm{C} ;{ }^{1} \mathrm{H}$ NMR $(400 \mathrm{MHz}$, $\left.\mathrm{CDCl}_{3}\right) \delta 8.20(\mathrm{~d}, J=3.6 \mathrm{~Hz}, 1 \mathrm{H}), 7.82(\mathrm{~d}, J=8.0 \mathrm{~Hz}, 1 \mathrm{H}), 7.77(\mathrm{~d}, J=7.6 \mathrm{~Hz}$, 1H), 7.54-7.49 (m, 3H), 7.39-7.34 (m, 3H), 7.31-7.27 (m, 1H), $7.19(\mathrm{dd}, J=8.0,1.6 \mathrm{~Hz}, 1 \mathrm{H}), 3.93(\mathrm{~s}$, $2 \mathrm{H}) ;{ }^{13} \mathrm{C}$ NMR $\left(101 \mathrm{MHz}, \mathrm{CDCl}_{3}\right) \delta 166.2,150.5,145.4,145.0,143.2,141.4,139.1,132.8,131.8,126.9$, $126.8,126.4,125.5,125.0,123.4,120.9,119.6,118.7,116.8,37.1 ; \mathrm{IR}(\mathrm{KBr})$ v 3055, 2962, 2923, 1617, 1596, 1582, 1455, 1440, 1400, 1261, 1095, 1066, 1024, 932, 911, 882, 802, 753, 739, $660 \mathrm{~cm}^{-1}$; HRMS (APCl) $m / z$ calculated for $\mathrm{C}_{20} \mathrm{H}_{14} \mathrm{NS}_{2}[\mathrm{M}+\mathrm{H}]^{+}: 332.0562$, found 332.0569 . 


\section{(Z)-N-Propyl-3H-benzo[c][1,2]dithiol-3-imine (2q)}

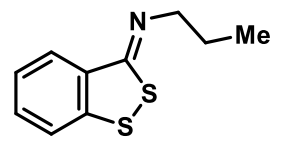

Yield: $62 \%$ (65 mg). Yellow oil; ${ }^{1} \mathrm{H}$ NMR $\left(400 \mathrm{MHz}, \mathrm{CDCl}_{3}\right) \delta 8.36-8.33(\mathrm{~m}, 1 \mathrm{H})$, 7.69-7.61 (m, 2H), 7.48-7.44 (m, 1H), 4.36-4.32 (m, 2H), 1.98-1.89 (m, 2H), 1.03 $(\mathrm{t}, J=7.2 \mathrm{~Hz}, 3 \mathrm{H}) ;{ }^{13} \mathrm{C} \mathrm{NMR}\left(101 \mathrm{MHz}, \mathrm{CDCl}_{3}\right) \delta 184.9,140.6,135.1,131.7,129.4,126.2,119.4,50.7$, 22.1, 11.1; IR (KBr) v 3058, 2964, 2930, 2872, 1594, 1561, 1458, 1443, 1371, 1351, 1324, 1301, 1241, 1131, 1090, 1069, 1016, 957, 764, $729 \mathrm{~cm}^{-1}$; HRMS (APCl) $\mathrm{m} / \mathrm{z}$ calculated for $\mathrm{C}_{10} \mathrm{H}_{12} \mathrm{NS}_{2}[\mathrm{M}+\mathrm{H}]^{+}$: 210.0406, found 210.0427 .

(Z)-5-Methyl-N-phenyl-3H-benzo[c][1,2]dithiol-3-imine (2r)<smiles>Cc1ccc2ss/c(=N\c3ccccc3)c2c1</smiles>

Yield: 87\% (112 mg). Yellow solid; mp $106-107^{\circ} \mathrm{C} ;{ }^{1} \mathrm{H}$ NMR $\left(400 \mathrm{MHz}, \mathrm{CDCl}_{3}\right) \delta$ $7.99(\mathrm{~s}, 1 \mathrm{H}), 7.45-7.40(\mathrm{~m}, 2 \mathrm{H}), 7.39-7.33(\mathrm{~m}, 2 \mathrm{H}), 7.21-7.17(\mathrm{~m}, 1 \mathrm{H}), 7.15-$ $7.13(\mathrm{~m}, 2 \mathrm{H}), 2.45$ (s, 3H); ${ }^{13} \mathrm{C} \mathrm{NMR}\left(101 \mathrm{MHz}, \mathrm{CDCl}_{3}\right) \delta 166.9,151.7,142.4,135.7,133.3,132.8,129.8$, $126.7,125.2,123.0,119.9,20.8 ; \mathrm{IR}(\mathrm{KBr})$ v 3052, 2951, 1610, 1585, 1482, 1467, 1383, 1308, 1244, 1201, 1138, 1067, 1011, 966, 844, 807, 787, 759, 698, $675 \mathrm{~cm}^{-1}$; HRMS (APCl) m/z calculated for $\mathrm{C}_{14} \mathrm{H}_{12} \mathrm{NS}_{2}[\mathrm{M}+\mathrm{H}]^{+}: 258.0406$, found 258.0416 .

(Z)-5-Methyl-N-(p-tolyl)-3H-benzo[c][1,2]dithiol-3-imine (2s)<smiles>Cc1ccc(N=c2ssc3ccc(C)cc23)cc1</smiles>
3H), 2.33 (s, 3H); $\left.{ }^{13} \mathrm{C} \mathrm{NMR} \mathrm{(101} \mathrm{MHz,} \mathrm{CDCl}_{3}\right) \delta 166.0,148.9,142.1,135.4,134.6,133.0,132.7,130.1$, 126.5, 122.8, 119.7, 21.0, 20.6; $\mathrm{IR}(\mathrm{KBr}) v$ 3018, 2918, 1892, 1589, 1500, 1466, 1401, 1271, 1242, 1200, 1154, 1137, 1105, 1064, 965, 855, 826, 809, 721, $664 \mathrm{~cm}^{-1}$; HRMS (APCI) $\mathrm{m} / \mathrm{z}$ calculated for 
$\mathrm{C}_{15} \mathrm{H}_{14} \mathrm{NS} 2[\mathrm{M}+\mathrm{H}]^{+}: 272.0562$, found 272.0585 .

(Z)-N-(4-Chlorophenyl)-5-methyl-3H-benzo[c][1,2]dithiol-3-imine (2t)

Yield: $83 \%(121 \mathrm{mg})$. Yellow solid; mp 115-116 ${ }^{\circ} \mathrm{C}^{1}{ }^{1} \mathrm{H} \mathrm{NMR}(400 \mathrm{MHz}$,

${ }^{13} \mathrm{C}$ NMR $\left(101 \mathrm{MHz}, \mathrm{CDCl}_{3}\right) \delta 167.5,150.1,142.5,135.9,133.5,132.7,130.2,129.9,126.7,123.0$, 121.4, 20.8; IR (KBr) v 2955, 2917, 1602, 1583, 1484, 1466, 1314, 1269, 1244, 1200, 1163, 1086, 1066, 1008, 961, 851, 829, 807, $692 \mathrm{~cm}^{-1}$; HRMS (APCI) $\mathrm{m} / \mathrm{z}$ calculated for $\mathrm{C}_{14} \mathrm{H}_{11} \mathrm{CINS}_{2}[\mathrm{M}+\mathrm{H}]^{+}: 292.0016$, found 292.0045 .

(Z)-5-Methoxy-N-phenyl-3H-benzo[c][1,2]dithiol-3-imine (2u)

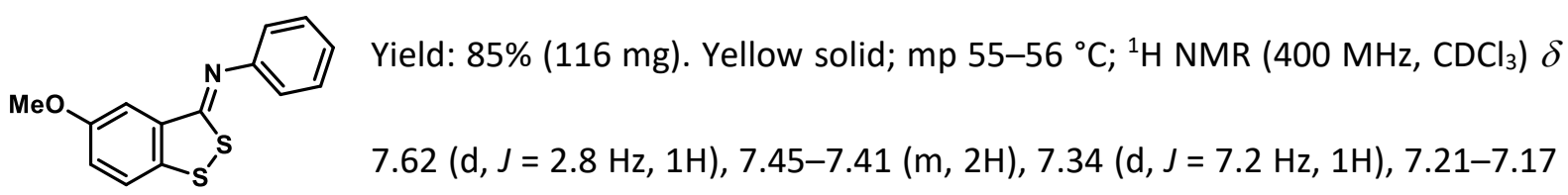
(m, 1H), 7.16-7.12 (m, 3H), $\left.3.89(\mathrm{~s}, 3 \mathrm{H}) ;{ }^{13} \mathrm{C} \mathrm{NMR} \mathrm{(101} \mathrm{MHz,} \mathrm{CDCl}_{3}\right) \delta 166.6,158.3,151.6,137.0$ 134.0, 129.8, 125.2, 123.8, 121.9, 119.9, 108.1, 55.8; IR (KBr) v 3059, 3000, 2957, 2832, 1600, 1585, $1471,1410,1337,1270,1216,1186,1157,1024,981,846,787,762,695,669 \mathrm{~cm}^{-1}$; HRMS (APCI) $m / z$ calculated for $\mathrm{C}_{14} \mathrm{H}_{12} \mathrm{NOS}_{2}[\mathrm{M}+\mathrm{H}]^{+}: 274.0355$, found 274.0384 .

(Z)-5-Methoxy- $N$-(p-tolyl)-3H-benzo[c][1,2]dithiol-3-imine (2v)

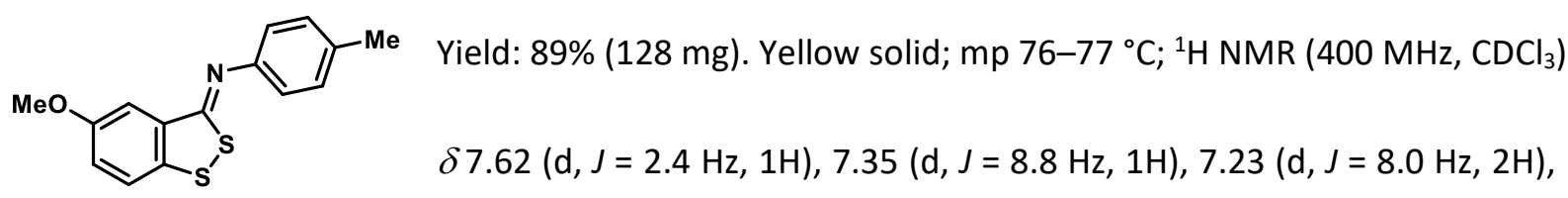

$7.14(\mathrm{dd}, J=8.8,2.8 \mathrm{~Hz}), 7.06(\mathrm{~d}, J=8.0 \mathrm{~Hz}, 2 \mathrm{H}), 3.90(\mathrm{~s}, 3 \mathrm{H}), 2.38(\mathrm{~s}, 3 \mathrm{H}) ;{ }^{13} \mathrm{C} \mathrm{NMR}\left(101 \mathrm{MHz}, \mathrm{CDCl}_{3}\right)$ 
$\delta 166.1,158.3,149.0,136.8,134.9,134.1,130.3,123.8,121.8,119.8,108.2,55.8,21.1 ; \mathrm{IR}(\mathrm{KBr}) v$ $3081,2959,2920,2851,1587,1504,1471,1419,1264,1213,1187,1175,1107,1080,1021,971$, $877,853,811,713,663 \mathrm{~cm}^{-1}$; HRMS (APCI) $\mathrm{m} / z$ calculated for $\mathrm{C}_{15} \mathrm{H}_{14} \mathrm{NOS}_{2}[\mathrm{M}+\mathrm{H}]^{+}: 288.0511$, found 288.0538 .

(Z)-N-(4-Chlorophenyl)-5-methoxy-3H-benzo[c][1,2]dithiol-3-imine (2w)

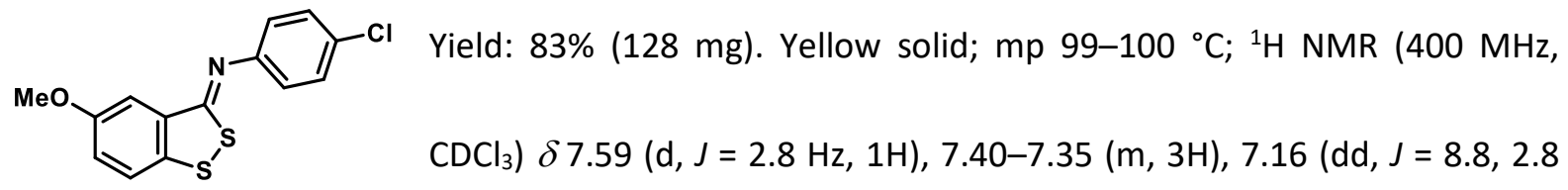

$\mathrm{Hz}, 1 \mathrm{H}), 7.11-7.07(\mathrm{~m}, 2 \mathrm{H}), 3.90(\mathrm{~s}, 3 \mathrm{H}) ;{ }^{13} \mathrm{C} \mathrm{NMR}\left(101 \mathrm{MHz}, \mathrm{CDCl}_{3}\right) \delta 167.2,158.4,150.0,137.1$, 133.9, 130.3, 129.9, 123.8, 122.1, 121.5, 108.1, 55.8; IR (KBr) v 3060, 3002, 2959, 2833, 1891, 1604, $1575,1470,1411,1341,1268,1217,1157,1088,1027,1009,984,858,833,808,714,667 \mathrm{~cm}^{-1}$; HRMS (APCI) $m / z$ calculated for $\mathrm{C}_{14} \mathrm{H}_{11} \mathrm{CINOS}_{2}[\mathrm{M}+\mathrm{H}]^{+}: 307.9965$, found 307.9987 .

(Z)-5-Fluoro-N-(p-tolyl)-3H-benzo[c][1,2]dithiol-3-imine (2x)

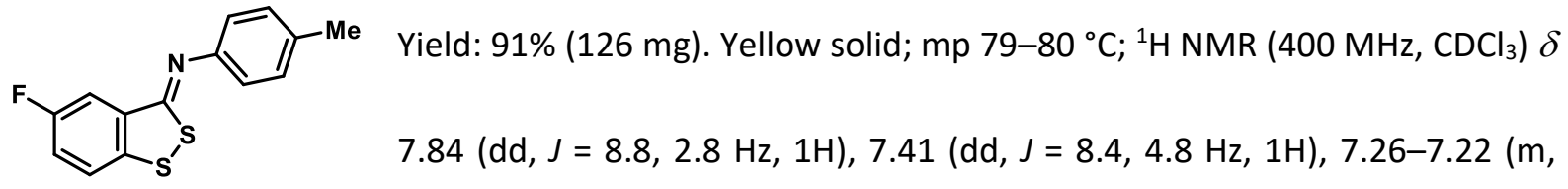
$3 \mathrm{H}), 7.06(\mathrm{~d}, J=8.0 \mathrm{~Hz}, 2 \mathrm{H}), 2.37(\mathrm{~s}, 3 \mathrm{H}) ;{ }^{13} \mathrm{C} \mathrm{NMR}\left(101 \mathrm{MHz}, \mathrm{CDCl}_{3}\right) \delta 164.3\left(\mathrm{~d}, J_{\mathrm{C}-\mathrm{F}}=4.0 \mathrm{~Hz}\right), 161.2(\mathrm{~d}$, $\left.J_{C-F}=245.2 \mathrm{~Hz}\right), 148.5,139.9\left(\mathrm{~d}, J_{\mathrm{C}-\mathrm{F}}=2.3 \mathrm{~Hz}\right), 135.2,135.1\left(\mathrm{~d}, J_{\mathrm{C}-\mathrm{F}}=8.2 \mathrm{~Hz}\right), 130.3,124.2\left(\mathrm{~d}, J_{\mathrm{C}-\mathrm{F}}=8.4\right.$ $\mathrm{Hz}), 119.9\left(\mathrm{~d}, J_{\mathrm{C}-\mathrm{F}}=24.8 \mathrm{~Hz}\right), 119.8,112.8\left(\mathrm{~d}, J_{\mathrm{C}-\mathrm{F}}=24.2 \mathrm{~Hz}\right), 21.1 ; \mathrm{IR}(\mathrm{KBr}) v 3060,2918,1888,1615$, $1588,1505,1459,1414,1315,1261,1192,1110,1069,986,893,859,808,713,660,643,607 \mathrm{~cm}^{-1}$; HRMS (APCl) $m / z$ calculated for $\mathrm{C}_{14} \mathrm{H}_{11} \mathrm{FNS}_{2}[\mathrm{M}+\mathrm{H}]^{+}: 276.0311$, found 276.0320 . 
(Z)-N-(4-Chlorophenyl)-5-fluoro-3H-benzo[c][1,2]dithiol-3-imine (2y)

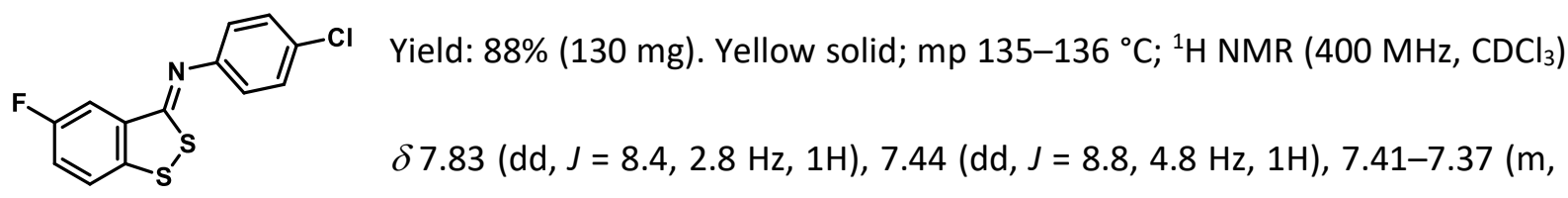
2H), 7.30-7.26 (m, 1H), 7.11-7.07 (m, 2H); $\left.{ }^{13} \mathrm{C} \mathrm{NMR} \mathrm{(101} \mathrm{MHz,} \mathrm{CDCl}_{3}\right) \delta 165.5\left(\mathrm{~d}, \mathrm{~J}_{\mathrm{C}-\mathrm{F}}=4.0 \mathrm{~Hz}\right), 161.2$ $\left(d, J_{C-F}=245.7 \mathrm{~Hz}\right), 149.5,140.2\left(d, J_{C-F}=2.3 \mathrm{~Hz}\right), 134.9\left(\mathrm{~d}, J_{C-F}=8.2 \mathrm{~Hz}\right), 130.6,129.9,124.3\left(\mathrm{~d}, J_{C-F}=\right.$ 8.4 Hz), 121.4, $120.2\left(\mathrm{~d}, J_{\mathrm{C}-\mathrm{F}}=24.7 \mathrm{~Hz}\right), 112.8\left(\mathrm{~d}, J_{\mathrm{C}-\mathrm{F}}=24.2 \mathrm{~Hz}\right) ; \mathrm{IR}(\mathrm{KBr}) v 3085,3065,1599,1581$, $1486,1461,1416,1320,1262,1192,1089,1071,1010,987,888,857,829,811,669 \mathrm{~cm}^{-1}$; HRMS (APCI) $m / z$ calculated for $\mathrm{C}_{13} \mathrm{H}_{8} \mathrm{ClFNS}_{2}[\mathrm{M}+\mathrm{H}]^{+}: 295.9765$, found 295.9790 .

(Z)-5-Chloro-N-phenyl-3H-benzo[c][1,2]dithiol-3-imine (2z)<smiles></smiles>

Yield: $82 \%$ (114 mg). Yellow solid; mp $88-89{ }^{\circ} \mathrm{C} ;{ }^{1} \mathrm{H} \mathrm{NMR}\left(400 \mathrm{MHz}, \mathrm{CDCl}_{3}\right) \delta$ 8.15-8.14 (m, 1H), 7.47-7.40 (m, 4H), 7.25-7.12 (m, 3H); ${ }^{13} \mathrm{C}$ NMR (101 MHz, $\left.\mathrm{CDCl}_{3}\right) \delta 164.7,151.1,143.4,134.5,132.0,131.9,129.8,126.4,125.5,124.1,119.8 ; \mathrm{IR}(\mathrm{KBr}) v 3063$, $1886,1616,1596,1578,1504,1448,1394,1238,1179,1166,1107,1080,955,886,850,814,777$, $565 \mathrm{~cm}^{-1}$; HRMS (APCI) $\mathrm{m} / z$ calculated for $\mathrm{C}_{13} \mathrm{H}_{9} \mathrm{ClNS}_{2}[\mathrm{M}+\mathrm{H}]^{+}: 277.9859$, found 277.9898 .

\section{(Z)-5-Chloro-N-(p-tolyl)-3H-benzo[c][1,2]dithiol-3-imine (2aa)}

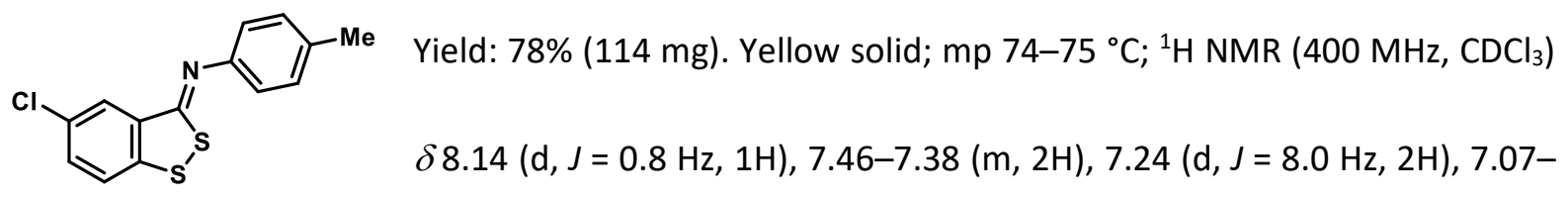

$7.04(\mathrm{~m}, 2 \mathrm{H}), 2.38(\mathrm{~s}, 3 \mathrm{H}) ;{ }^{13} \mathrm{C} \mathrm{NMR}\left(101 \mathrm{MHz}, \mathrm{CDCl}_{3}\right) \delta 164.0,148.5,143.3,135.3,134.7,132.0,131.8$, $130.3,126.4,124.1,119.8,21.1 ; \operatorname{IR}(\mathrm{KBr})$ v 3059, 2920, 1933, 1606, 1590, 1575, 1484, 1453, 1447, 1397, 1238, 1193, 1164, 1080, 957, 887, 837, 820, 763, 689, $669 \mathrm{~cm}^{-1}$; HRMS (APCl) $\mathrm{m} / \mathrm{z}$ calculated 
for $\mathrm{C}_{14} \mathrm{H}_{11} \mathrm{CINS} 2[\mathrm{M}+\mathrm{H}]^{+}:$292.0016, found 292.0046 .

(Z)-5-Chloro-N-(4-chlorophenyl)-3H-benzo[c][1,2]dithiol-3-imine (2ab)

Yield: $90 \%(140 \mathrm{mg})$. Yellow solid; mp $141-142{ }^{\circ} \mathrm{C} ;{ }^{1} \mathrm{H}$ NMR $(400 \mathrm{MHz}$, 7.10-7.07 (m, 2H); $\left.{ }^{13} \mathrm{C} \mathrm{NMR} \mathrm{(101} \mathrm{MHz,} \mathrm{CDCl}_{3}\right) \delta 165.3,149.4,143.5,134.4,132.2,132.1,130.6,129.9$, $126.4,124.1,121.3$; IR (KBr) v 3081, 3058, 1603, 1580, 1485, 1452, 1398, 1309, 1243, 1178, 1163, $1083,1012,956,892,842,828,813,756,684 \mathrm{~cm}^{-1}$; HRMS (APCl) $\mathrm{m} / \mathrm{z}$ calculated for $\mathrm{C}_{13} \mathrm{H}_{8} \mathrm{Cl}_{2} \mathrm{NS}_{2}[\mathrm{M}+$ $\mathrm{H}]^{+}:$311.9470, found 311.9493 .

(Z)-7-Methyl-N-(p-tolyl)-3H-benzo[c][1,2]dithiol-3-imine (2ac)

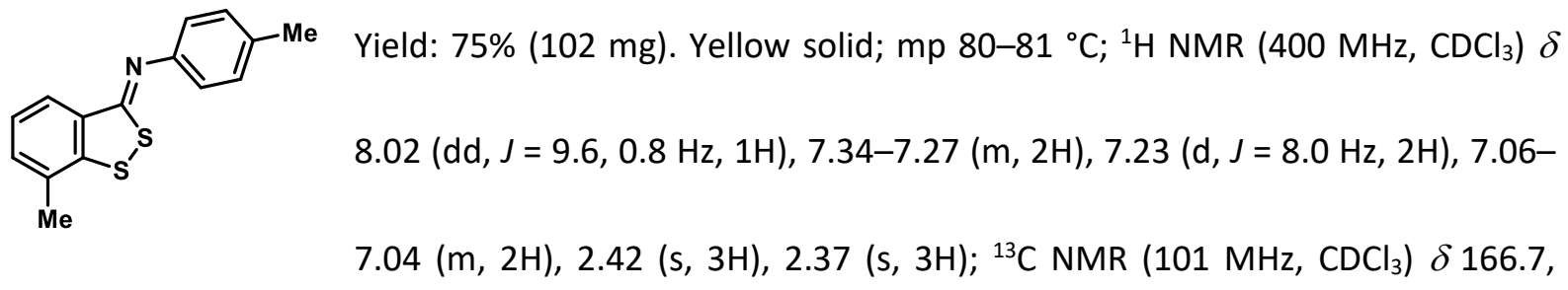

$149.1,145.3,134.8,132.9,132.7,132.1,130.3,126.0,124.5,119.8,21.1,19.5 ; \mathrm{IR}(\mathrm{KBr})$ v 3044, 3027, $2934,2917,1899,1605,1502,1449,1382,1310,1255,1198,1102,1051,1020,964,828,779,712$, $698 \mathrm{~cm}^{-1}$; HRMS (APCI) $\mathrm{m} / z$ calculated for $\mathrm{C}_{15} \mathrm{H}_{14} \mathrm{NS}_{2}[\mathrm{M}+\mathrm{H}]^{+}: 272.0562$, found 272.0586 . 


\section{(Z)-N-(4-Chlorophenyl)-7-methyl-3H-benzo[c][1,2]dithiol-3-imine (2ad)}

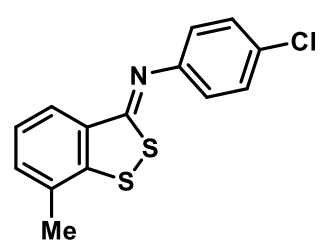

Yield: 78\% (114 mg). Yellow solid; mp $121-122{ }^{\circ} \mathrm{C} ;{ }^{1} \mathrm{H}$ NMR (400 MHz, $\left.\mathrm{CDCl}_{3}\right) \delta$ $8.00(\mathrm{dd}, J=7.6,0.8 \mathrm{~Hz}, 1 \mathrm{H}), 7.40-7.29(\mathrm{~m}, 4 \mathrm{H}), 7.10-7.06(\mathrm{~m}, 2 \mathrm{H}), 2.43(\mathrm{~s}, 3 \mathrm{H})$;

${ }^{13} \mathrm{C} \mathrm{NMR}\left(101 \mathrm{MHz}, \mathrm{CDCl}_{3}\right) \delta 167.8,150.0,145.6,132.9,132.5,132.3,130.2$, $129.8,126.1,124.5,121.5,19.5 ; \mathrm{IR}(\mathrm{KBr})$ v 3050, 2978, 2929, 1882, 1604, 1580, 1481, 1408, 1306, 1197, 1086, 1052, 1006, 965, 868, 825, 789, 718, 709, $640 \mathrm{~cm}^{-1}$; HRMS (APCl) $\mathrm{m} / \mathrm{z}$ calculated for $\mathrm{C}_{14} \mathrm{H}_{11} \mathrm{ClNS}_{2}[\mathrm{M}+\mathrm{H}]^{+}:$292.0016, found 292.0045.

(Z)-N-(4-Chlorophenyl)-3H-[1,2]dithiolo[4,3-b]pyridin-3-imine (2ae)

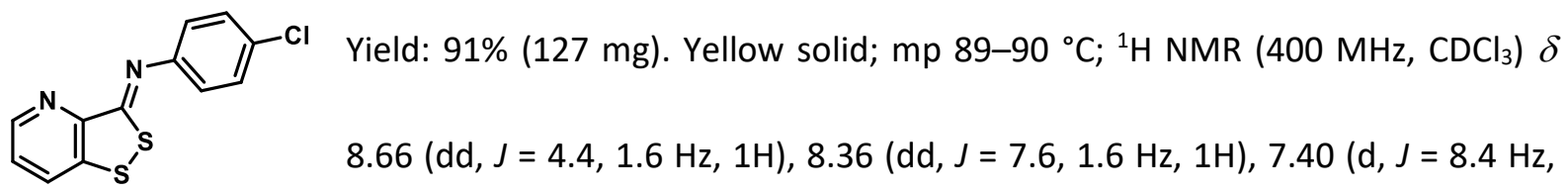
$2 \mathrm{H}), 7.33-7.30(\mathrm{~m}, 1 \mathrm{H}), 7.08(\mathrm{~d}, J=8.8 \mathrm{~Hz}, 2 \mathrm{H}) ;{ }^{13} \mathrm{C} \mathrm{NMR}\left(101 \mathrm{MHz}, \mathrm{CDCl}_{3}\right) \delta 166.2,162.3,152.3$, $147.5,133.7,129.8,128.9,125.4,120.4,119.0$; IR (KBr) v 3058, 1605, 1558, 1486, 1458, 1394, 1261, 1235, 1210, 1166, 1093, 1023, 919, 828, 805, 740, 710, $675 \mathrm{~cm}^{-1}$; HRMS (APCI) $\mathrm{m} / \mathrm{z}$ calculated for $\mathrm{C}_{12} \mathrm{H}_{8} \mathrm{ClN}_{2} \mathrm{~S}_{2}[\mathrm{M}+\mathrm{H}]^{+}:$278.9812, found 278.9840 .

\section{$3 H-B e n z o[c][1,2]$ dithiol-3-one $(3 a)^{3}$}<smiles></smiles>

Yield: 81\% (68 mg). Yellow solid; mp 77-78 ${ }^{\circ} \mathrm{C} ;{ }^{1} \mathrm{H}$ NMR (400 MHz, $\left.\mathrm{CDCl}_{3}\right) \delta 7.96$ (d, $J=8.0$ $\mathrm{Hz}, 1 \mathrm{H}), 7.68-7.62(\mathrm{~m}, 2 \mathrm{H}), 7.43-7.39(\mathrm{~m}, 1 \mathrm{H}) ;{ }^{13} \mathrm{C}$ NMR $\left(101 \mathrm{MHz}, \mathrm{CDCl}_{3}\right) \delta 193.6,148.3$, 133.5, 129.2, 127.4, 125.6, 124.7; HRMS (APCI) $\mathrm{m} / z$ calculated for $\mathrm{C}_{7} \mathrm{H}_{5} \mathrm{OS}_{2}[\mathrm{M}+\mathrm{H}]^{+}: 168.9775$, found 168.9782. 


\section{5-Methyl-3H-benzo[c][1,2]dithiol-3-one (3b) ${ }^{4}$}

Me Yield: $86 \%$ (79 mg). White solid; mp 73-74 ${ }^{\circ} \mathrm{C}$; ${ }^{1} \mathrm{H} \mathrm{NMR}\left(400 \mathrm{MHz}, \mathrm{CDCl}_{3}\right) \delta 7.74$ (s, 1H), 7.51-7.45 (m, 2H), $2.45(\mathrm{~s}, 3 \mathrm{H}) ;{ }^{13} \mathrm{C}$ NMR (101 MHz, $\left.\mathrm{CDCl}_{3}\right) \delta 193.6,145.4,135.9$, 135.0, 129.3, 126.9, 124.2, 20.7; HRMS (APCI) $\mathrm{m} / \mathrm{z}$ calculated for $\mathrm{C}_{8} \mathrm{H}_{7} \mathrm{OS}_{2}[\mathrm{M}+\mathrm{H}]^{+}: 182.9933$, found 182.9947.

\section{5-Methoxy-3H-benzo[c][1,2]dithiol-3-one (3c)}

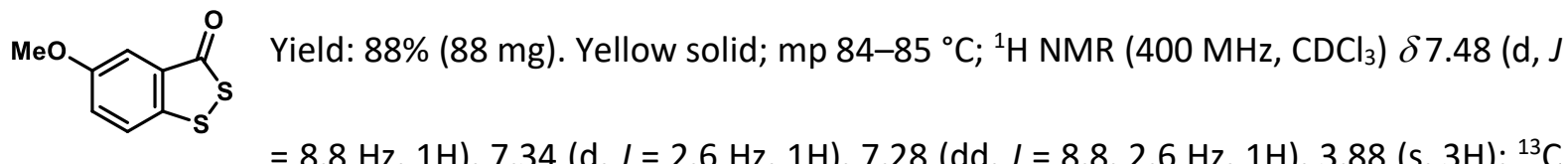
NMR (101 MHz, $\left.\mathrm{CDCl}_{3}\right) \delta 193.2,158.3,140.2,130.5,124.9,124.1,107.7,55.8 ;$ HRMS (APCI) $\mathrm{m} / \mathrm{z}$ calculated for $\mathrm{C}_{8} \mathrm{H}_{7} \mathrm{O}_{2} \mathrm{~S}_{2}[\mathrm{M}+\mathrm{H}]^{+}:$198.9882, found 198.9897 .

\section{5-Fluoro-3H-benzo[c][1,2]dithiol-3-one (3d)}<smiles></smiles>

Yield: $69 \%$ (65 mg). White solid; $\mathrm{mp} 77-78^{\circ} \mathrm{C} ;{ }^{1} \mathrm{H} \mathrm{NMR}\left(400 \mathrm{MHz}, \mathrm{CDCl}_{3}\right) \delta 7.62-7.57$ (m, 2H), 7.45-7.40 (m, 1H); $\left.{ }^{13} \mathrm{C} \mathrm{NMR} \mathrm{(101} \mathrm{MHz,} \mathrm{CDCl}\right) \delta 192.1\left(\mathrm{~d}, J_{\mathrm{C}-\mathrm{F}}=3.0 \mathrm{~Hz}\right), 162.9$ $\left(d, J_{C-F}=247.7 \mathrm{~Hz}\right), 143.1\left(\mathrm{~d}, J_{\mathrm{C}-\mathrm{F}}=1.2 \mathrm{~Hz}\right), 131.0\left(\mathrm{~d}, J_{\mathrm{C}-\mathrm{F}}=7.6 \mathrm{~Hz}\right), 125.6,\left(\mathrm{~d}, J_{\mathrm{C}-\mathrm{F}}=8.3 \mathrm{~Hz}\right), 122.0\left(\mathrm{~d}, J_{\mathrm{C}-}\right.$ $\mathrm{F}=25.0 \mathrm{~Hz}), 112.8\left(\mathrm{~d}, \mathrm{~J}_{\mathrm{C}-\mathrm{F}}=23.5 \mathrm{~Hz}\right) ; \mathrm{HRMS}(\mathrm{APCl}) \mathrm{m} / \mathrm{z}$ calculated for $\mathrm{C}_{7} \mathrm{H}_{4} \mathrm{FOS}_{2}[\mathrm{M}+\mathrm{H}]^{+}: 186.9688$, found 186.9692 . 


\section{5-Chloro-3H-benzo[c][1,2]dithiol-3-one (3e)}<smiles></smiles>

Yield: $74 \%$ (75 mg). White solid; mp $123-124{ }^{\circ} \mathrm{C} ;{ }^{1} \mathrm{H} \mathrm{NMR}\left(400 \mathrm{MHz}, \mathrm{CDCl}_{3}\right) \delta 7.90(\mathrm{~d}$, $J=2.0 \mathrm{~Hz}, 1 \mathrm{H}), 7.62-7.54(\mathrm{~m}, 2 \mathrm{H}) ;{ }^{13} \mathrm{C} \mathrm{NMR}\left(101 \mathrm{MHz}, \mathrm{CDCl}_{3}\right) \delta 191.9,146.2,133.6$, 132.3, 130.6, 126.7, 125.4; HRMS (APCl) $\mathrm{m} / \mathrm{z}$ calculated for $\mathrm{C}_{7} \mathrm{H}_{4} \mathrm{ClOS}_{2}[\mathrm{M}+\mathrm{H}]^{+}:$202.9392, found 202.9399.

\section{$3 H$-Benzo[c][1,2]dithiol-3-one 1,1-dioxide $(4)^{5}$}<smiles>O=C1SS(=O)(=O)c2ccccc21</smiles>

Yield: $71 \%$ (71 mg). White solid; mp 96-97 ${ }^{\circ} \mathrm{C} ;{ }^{1} \mathrm{H} \mathrm{NMR}\left(400 \mathrm{MHz}, \mathrm{CDCl}_{3}\right) \delta 8.13$ (dd, $J=$ 8.0, $0.4 \mathrm{~Hz}, 1 \mathrm{H}), 8.05-7.96(\mathrm{~m}, 2 \mathrm{H}), 7.90-7.86(\mathrm{~m}, 1 \mathrm{H}) ;{ }^{13} \mathrm{C} \mathrm{NMR}\left(101 \mathrm{MHz}, \mathrm{CDCl}_{3}\right) \delta$ $182.9,148.5,136.3,134.4,130.1,125.6,121.9 ;$ HRMS $(A P C l) m / z$ calculated for $\mathrm{C}_{7} \mathrm{H}_{5} \mathrm{O}_{3} \mathrm{~S}_{2}[\mathrm{M}+\mathrm{H}]^{+}$: 200.9675 , found 200.9679 .

\section{$3 H$-Benzo[c][1,2]dithiol-3-one 1-oxide $(5)^{6}$}

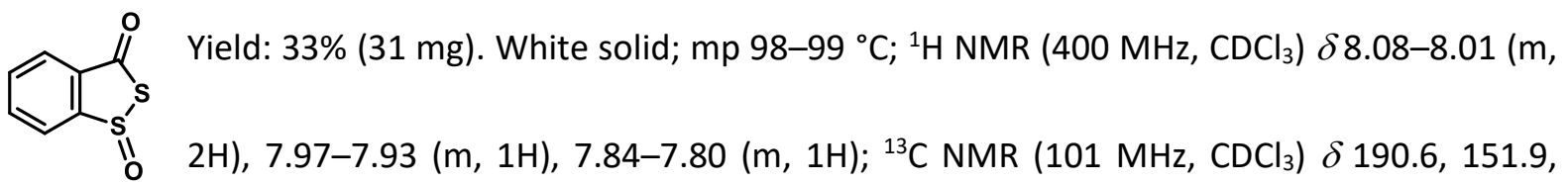
135.7, 133.2, 131.2, 128.1, 127.0; HRMS (APCI) $\mathrm{m} / \mathrm{z}$ calculated for $\mathrm{C}_{7} \mathrm{H}_{5} \mathrm{O}_{2} \mathrm{~S}_{2}[\mathrm{M}+\mathrm{H}]^{+}:$184.9725, found 184.9731 .

(Z)-N-Phenyl-3H-benzo[d][1,2]thiaselenol-3-imine (6a)

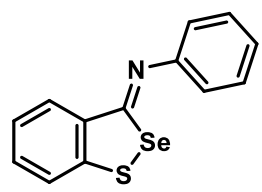

Yield: $69 \%$ (101 mg). Yellow solid; $\mathrm{mp} 61-62{ }^{\circ} \mathrm{C} ;{ }^{1} \mathrm{H} \mathrm{NMR}\left(400 \mathrm{MHz}, \mathrm{CDCl}_{3}\right) \delta 8.20$ (d, $J=8.0 \mathrm{~Hz}, 1 \mathrm{H}), 7.49-7.43(\mathrm{~m}, 4 \mathrm{H}), 7.29-7.21(\mathrm{~m}, 2 \mathrm{H}), 7.14(\mathrm{~d}, J=8.0 \mathrm{~Hz}, 2 \mathrm{H})$;

${ }^{13} \mathrm{C}$ NMR $\left(101 \mathrm{MHz}, \mathrm{CDCl}_{3}\right) \delta 168.1,153.4,146.7,135.2,131.8,130.0,127.4,125.8,125.1,124.6$, 
119.0; IR (KBr) v 3056, 1619, 1584, 1482, 1460, 1435, 1270, 1240, 1191, 1166, 1152, 1064, 1024, 911, 887, 830, 756, $693 \mathrm{~cm}^{-1}$; HRMS (APCI) $\mathrm{m} / \mathrm{z}$ calculated for $\mathrm{C}_{13} \mathrm{H}_{10} \mathrm{NSSe}[\mathrm{M}+\mathrm{H}]^{+}: 291.9693$, found 291.9713.

(Z)- $N$-(p-Tolyl)-3H-benzo[d][1,2]thiaselenol-3-imine (6b)

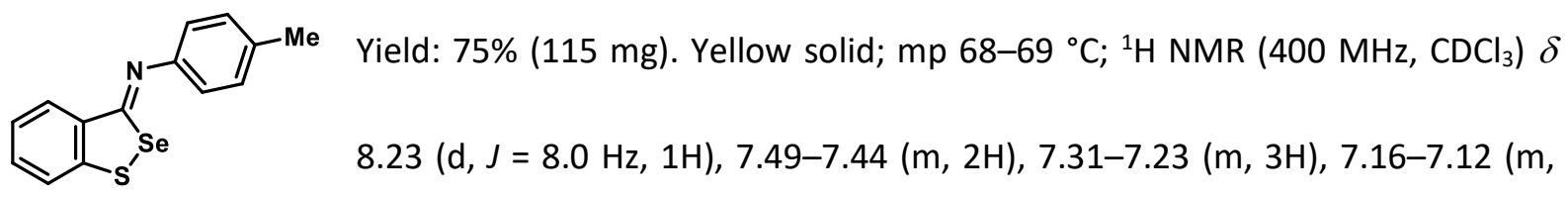
1H), $7.03(\mathrm{~d}, J=8.0 \mathrm{~Hz}, 1 \mathrm{H}), 2.25(\mathrm{~s}, 3 \mathrm{H}) ;{ }^{13} \mathrm{C} \mathrm{NMR}\left(101 \mathrm{MHz}, \mathrm{CDCl}_{3}\right) \delta 167.5,150.9,146.6,135.5$, 135.3, 131.7, 130.6, 127.4, 125.1, 124.6, 118.8, 21.1; IR (KBr) v 3049, 3018, 2916, 2856, 1618, 1598, $1501,1460,1434,1306,1270,1246,1168,1106,1064,1014,956,898,817,758 \mathrm{~cm}^{-1}$; HRMS (APCI) $m / z$ calculated for $\mathrm{C}_{14} \mathrm{H}_{12} \mathrm{NSSe}[\mathrm{M}+\mathrm{H}]^{+}: 305.9850$, found 305.9879 .

(Z)-N-(4-Isopropylphenyl)-3H-benzo[d][1,2]thiaselenol-3-imine (6c)

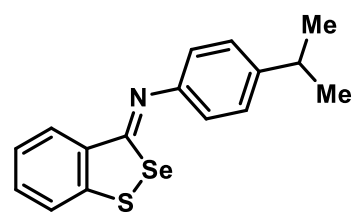

Yield: 76\% (127 mg). Yellow solid; mp $43-44{ }^{\circ} \mathrm{C} ;{ }^{1} \mathrm{H}$ NMR (400 MHz, $\left.\mathrm{CDCl}_{3}\right) \delta$ 8.20-8.18 (m, 1H), 7.49-7.42 (m, 2H), 7.31-7.23 (m, 3H), 7.10-7.07 (m, 2H), 2.98-2.91 (m, 1H), $1.29(\mathrm{~s}, 3 \mathrm{H}), 1.27(\mathrm{~s}, 3 \mathrm{H}) ;{ }^{13} \mathrm{C} \mathrm{NMR}\left(101 \mathrm{MHz}, \mathrm{CDCl}_{3}\right) \delta$ 167.2, 151.0, 146.5, 135.3, 131.6, 127.9, 127.5, 125.0, 124.6, 118.8, 33.7, 24.0; IR (KBr) v 3053, 2957, $2867,1613,1596,1501,1459,1434,1381,1362,1268,1243,1191,1112,1065,1013,897,834,755$, $719 \mathrm{~cm}^{-1}$; HRMS (APCI) $\mathrm{m} / z$ calculated for $\mathrm{C}_{16} \mathrm{H}_{16} \mathrm{NSSe}[\mathrm{M}+\mathrm{H}]^{+}: 334.0163$, found 334.0165 . 
(Z)-N-(4-(tert-Butyl)phenyl)-3H-benzo[d][1,2]thiaselenol-3-imine (6d)

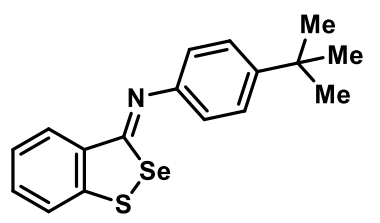

Yield: $72 \%$ (125 mg). Pale yellow oil; ${ }^{1} \mathrm{H}$ NMR $\left(400 \mathrm{MHz}, \mathrm{CDCl}_{3}\right) \delta 8.19(\mathrm{~d}, J=$ 4.4 Hz, 1H), 7.48-7.41 (m, 4H), 7.27-7.23 (m, 1H), 7.10-7.08 (m, 2H), 1.35

(s, 9H); ${ }^{13} \mathrm{C}$ NMR $\left(101 \mathrm{MHz}, \mathrm{CDCl}_{3}\right) \delta 167.1,150.6,148.8,146.5,135.4,131.7$, 127.5, 126.8, 125.1, 124.6, 118.6, 34.6, 31.4; IR (KBr) v 3055, 2960, 2864, 1610, 1595, 1582, 1504, 1459, 1434, 1362, 1268, 1244, 1173, 1115, 1065, 1012, 894, 834, 755, $719 \mathrm{~cm}^{-1}$; HRMS (APCI) $\mathrm{m} / \mathrm{z}$ calculated for $\mathrm{C}_{17} \mathrm{H}_{18} \mathrm{NSSe}[\mathrm{M}+\mathrm{H}]^{+}: 348.0320$, found 348.0331 .

(Z)-N-(4-Methoxyphenyl)-3H-benzo[d][1,2]thiaselenol-3-imine (6e)

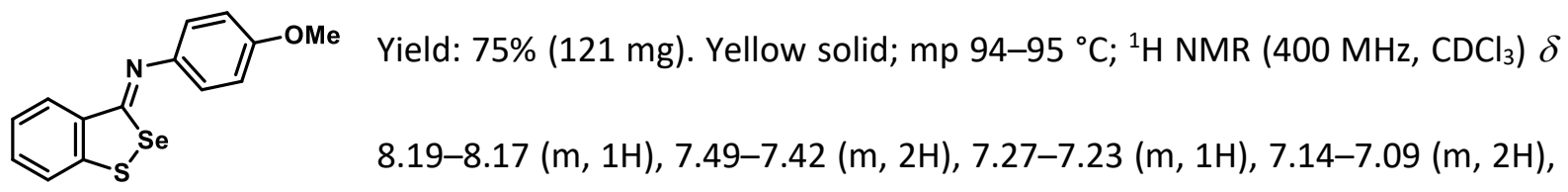
7.00-6.96 (m, 2H), 3.85 (s, 3H); $\left.{ }^{13} \mathrm{C} \mathrm{NMR} \mathrm{(101} \mathrm{MHz,} \mathrm{CDCl}_{3}\right) \delta 167.3,157.5,146.7,146.4,135.3,131.6$, 127.4, 125.1, 124.6, 120.3, 115.1, 55.5; IR (KBr) v 3047, 2994, 2952, 2833, 1615, 1597, 1561, 1503, $1460,1436,1294,1239,1188,1107,1039,896,830,757,752,720 \mathrm{~cm}^{-1}$; HRMS (APCI) m/z calculated for $\mathrm{C}_{14} \mathrm{H}_{12} \mathrm{NOSSe}[\mathrm{M}+\mathrm{H}]^{+}: 321.9799$, found 321.9811 .

(Z)-N-(4-Ethoxyphenyl)-3H-benzo[d][1,2] thiaselenol-3-imine (6f)

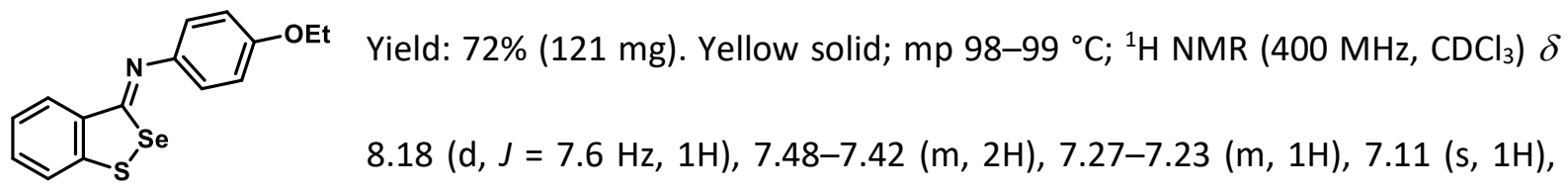

$7.09(\mathrm{~s}, 1 \mathrm{H}), 6.98-6.94(\mathrm{~m}, 2 \mathrm{H}), 4.06(\mathrm{q}, J=6.8 \mathrm{~Hz}, 2 \mathrm{H}), 1.44(\mathrm{t}, J=6.8 \mathrm{~Hz}, 3 \mathrm{H}) ;{ }^{13} \mathrm{C} \mathrm{NMR}(101 \mathrm{MHz}$, $\left.\mathrm{CDCl}_{3}\right) \delta 168.7,149.2,146.8,142.7,135.1,131.6,127.6,126.6,125.0,124.7,121.6,119.4,114.2$ 64.6, 14.8; IR (KBr) v 3063, 3029, 2977, 2922, 2877, 1612, 1499, 1471, 1433, 1395, 1289, 1270, 1246, 
$1236,1162,1117,1047,919,893,763 \mathrm{~cm}^{-1}$; HRMS (APCl) $\mathrm{m} / z$ calculated for $\mathrm{C}_{15} \mathrm{H}_{14} \mathrm{NOSSe}[\mathrm{M}+\mathrm{H}]^{+}$: 335.9955 , found 335.9962 .

(Z)-N-(4-Chlorophenyl)-3H-benzo[d][1,2]thiaselenol-3-imine (6g)

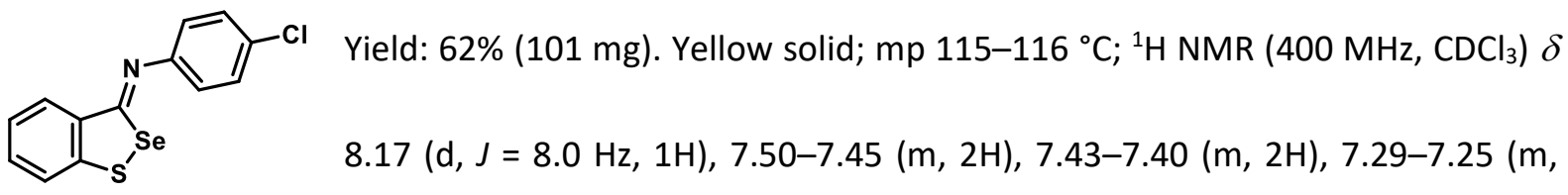
1H), 7.10-7.06 (m, 2H); $\left.{ }^{13} \mathrm{C} \mathrm{NMR} \mathrm{(101} \mathrm{MHz,} \mathrm{CDCl}_{3}\right) \delta 168.9,151.7,146.8,135.1,131.9,130.9,130.2$, 127.4, 125.2, 124.7, 120.5; IR (KBr) v 3057, 1625, 1615, 1583, 1561, 1482, 1437, 1398, 1272, 1242, 1187, 1159, 1120, 1084, 1066, 1008, 901, 825, 758, $673 \mathrm{~cm}^{-1}$; HRMS (APCI) $\mathrm{m} / \mathrm{z}$ calculated for $\mathrm{C}_{13} \mathrm{H}_{9} \mathrm{ClNSSe}[\mathrm{M}+\mathrm{H}]^{+}: 325.9303$, found 325.9305.

\section{(Z)-N-(4-Fluorophenyl)-3H-benzo[d][1,2]thiaselenol-3-imine (6h)}

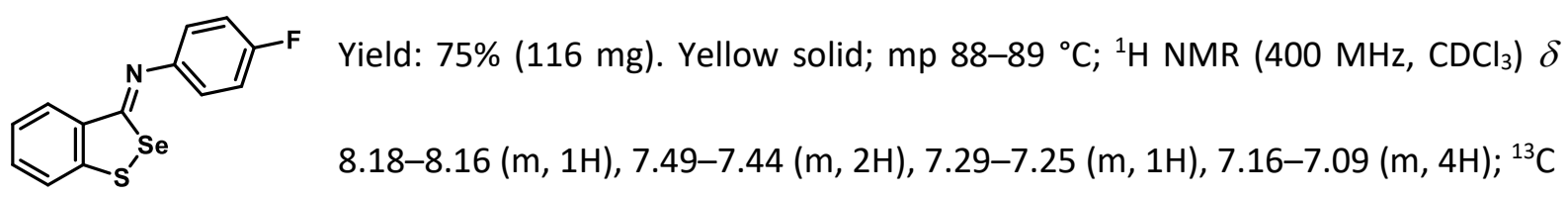

$\operatorname{NMR}\left(101 \mathrm{MHz}, \mathrm{CDCl}_{3}\right) \delta 168.7,160.4\left(\mathrm{~d}, J_{\mathrm{C}-\mathrm{F}}=243.2 \mathrm{~Hz}\right), 149.4\left(\mathrm{~d}, J_{\mathrm{C}-\mathrm{F}}=2.5 \mathrm{~Hz}\right), 146.7,135.0,131.9$, 127.4, 125.2, 124.6, $120.6\left(\mathrm{~d}, J_{\mathrm{C}-\mathrm{F}}=8.3 \mathrm{~Hz}\right), 116.8\left(\mathrm{~d}, J_{\mathrm{C}-\mathrm{F}}=22.7 \mathrm{~Hz}\right) ; \mathrm{IR}(\mathrm{KBr}) v 3058,1613,1561,1495$, 1458, 1434, 1306, 1271, 1217, 1185, 1088, 1063, 1010, 934, 900, 836, 773, 761, $721 \mathrm{~cm}^{-1}$; HRMS (APCI) $m / z$ calculated for $\mathrm{C}_{13} \mathrm{H}_{9} \mathrm{FNSSe}[\mathrm{M}+\mathrm{H}]^{+}:$309.9599, found 309.9615. 


\section{(Z)-N-(m-Tolyl)-3H-benzo[d][1,2]thiaselenol-3-imine (6i)}

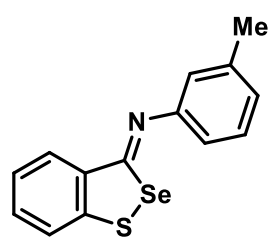

Yield: 71\% (109 mg). Yellow solid; mp 33-34 ${ }^{\circ} \mathrm{C} ;{ }^{1} \mathrm{H}$ NMR $\left(400 \mathrm{MHz}, \mathrm{CDCl}_{3}\right) \delta 8.18-$ $8.16(m, 1 H), 7.47-7.40(m, 2 H), 7.34-7.30(m, 1 H), 7.26-7.22(m, 1 H), 7.04-7.02$ $(\mathrm{m}, 1 \mathrm{H}), 6.95(\mathrm{~s}, 1 \mathrm{H}), 6.94(\mathrm{~s}, 1 \mathrm{H}), 2.38(\mathrm{~s}, 3 \mathrm{H}) ;{ }^{13} \mathrm{C} \mathrm{NMR}\left(101 \mathrm{MHz}, \mathrm{CDCl}_{3}\right) \delta 167.7$, $153.4,146.6,140.0,135.2,131.7,129.8,127.4,126.5,125.0,124.6,119.6,115.6,21.5 ; \mathrm{IR}(\mathrm{KBr}) v$ $3053,2953,2916,1613,1595,1578,1481,1460,1433,1254,1207,1147,1064,930,907,889,785$, $752,719,697 \mathrm{~cm}^{-1}$; HRMS (APCl) $\mathrm{m} / z$ calculated for $\mathrm{C}_{14} \mathrm{H}_{12} \mathrm{NSSe}[\mathrm{M}+\mathrm{H}]^{+}: 305.9850$, found 305.9879 .

(Z)-N-(3-Chlorophenyl)-3H-benzo[d][1,2]thiaselenol-3-imine (6j)

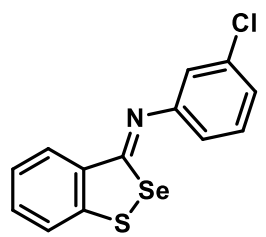

Yield: 68\% (111 mg). Yellow solid; mp 80-81 ${ }^{\circ} \mathrm{C} ;{ }^{1} \mathrm{H}$ NMR (400 MHz, $\left.\mathrm{CDCl}_{3}\right) \delta 8.18-$ $8.16(\mathrm{~m}, 1 \mathrm{H}), 7.51-7.46(\mathrm{~m}, 2 \mathrm{H}), 7.38(\mathrm{t}, J=8.0 \mathrm{~Hz}, 1 \mathrm{H}), 7.30-7.27(\mathrm{~m}, 1 \mathrm{H}), 7.22-$ $7.19(\mathrm{~m}, 1 \mathrm{H}), 7.14(\mathrm{t}, \mathrm{J}=2.0 \mathrm{~Hz}, 1 \mathrm{H}), 7.04-7.02(\mathrm{~m}, 1 \mathrm{H}) ;{ }^{13} \mathrm{C} \mathrm{NMR}\left(101 \mathrm{MHz}, \mathrm{CDCl}_{3}\right)$ $\delta 169.4,154.3,146.9,135.5,135.1,132.0,131.2,127.5,125.7,125.3,124.7,119.6,117.1 ; \mathrm{IR}(\mathrm{KBr}) v$ $3050,1607,1580,1560,1467,1434,1308,1259,1182,1154,1089,1067,1027,909,881,752,689$ $\mathrm{cm}^{-1}$; HRMS (APCl) $\mathrm{m} / z$ calculated for $\mathrm{C}_{13} \mathrm{H}_{9} \mathrm{ClNSSe}[\mathrm{M}+\mathrm{H}]^{+}: 325.9303$, found 325.9306 .

(Z)-N-(o-Tolyl)-3H-benzo[d][1,2]thiaselenol-3-imine (6k)

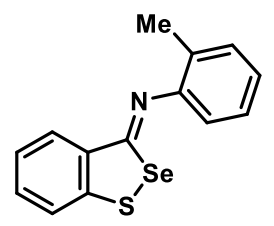

Yield: $72 \%$ (110 mg). Yellow solid; $\mathrm{mp} 88-89{ }^{\circ} \mathrm{C} ;{ }^{1} \mathrm{H} \mathrm{NMR}\left(400 \mathrm{MHz}, \mathrm{CDCl}_{3}\right) \delta 8.18$ $(\mathrm{d}, J=8.0 \mathrm{~Hz}, 1 \mathrm{H}), 7.48-7.42(\mathrm{~m}, 2 \mathrm{H}), 7.27-7.23(\mathrm{~m}, 3 \mathrm{H}), 7.06(\mathrm{~s}, 1 \mathrm{H}), 7.04(\mathrm{~s}, 1 \mathrm{H})$, $2.39(\mathrm{~s}, 3 \mathrm{H}) ;{ }^{13} \mathrm{C} \mathrm{NMR}\left(101 \mathrm{MHz}, \mathrm{CDCl}_{3}\right) \delta 167.6,152.2,146.8,134.9,131.7,131.3$ 129.1, 127.4, 127.4, 125.7, 125.1, 124.7, 116.8, 17.6; IR (KBr) v 3064, 3015, 2972, 2918, 1629, 1573, 1482, 1460, 1432, 1372, 1269, 1239, 1178, 1104, 1065, 1047, 931, 899, 751, $717 \mathrm{~cm}^{-1}$; HRMS (APCI) 
$\mathrm{m} / \mathrm{z}$ calculated for $\mathrm{C}_{14} \mathrm{H}_{12} \mathrm{NSSe}[\mathrm{M}+\mathrm{H}]^{+}:$305.9850, found 305.9878 .

(Z)-N-(2-Ethoxyphenyl)-3H-benzo[d][1,2]thiaselenol-3-imine (6I)<smiles>CCOc1ccccc1/N=c1\[cH-]sc2ccccc12</smiles>

Yield: $66 \%$ (111 mg). Yellow solid; $\mathrm{mp} 57-58{ }^{\circ} \mathrm{C} ;{ }^{1} \mathrm{H} \mathrm{NMR}\left(400 \mathrm{MHz}, \mathrm{CDCl}_{3}\right) \delta 8.25$ $(\mathrm{d}, J=8.0 \mathrm{~Hz}, 1 \mathrm{H}), 7.49-7.43(\mathrm{~m}, 2 \mathrm{H}), 7.29-7.25(\mathrm{~m}, 1 \mathrm{H}), 7.20-7.16(\mathrm{~m}, 1 \mathrm{H}), 7.08$ (dd, $J=7.6,2.0 \mathrm{~Hz}, 1 \mathrm{H}), 7.03-6.98(\mathrm{~m}, 2 \mathrm{H}), 4.10(\mathrm{q}, J=7.0 \mathrm{~Hz}, 2 \mathrm{H}), 1.37(\mathrm{t}, J=7.0$ $\mathrm{Hz}, 3 \mathrm{H}) ;{ }^{13} \mathrm{C}$ NMR $\left(101 \mathrm{MHz}, \mathrm{CDCl}_{3}\right) \delta 168.7,149.2,146.8,142.7,135.1,131.6,127.6,126.6,125.0$, 124.7, 121.6, 119.4, 114.2, 64.6, 14.8; IR (KBr) v 3050, 2975, 2925, 1599, 1578, 1493, 1472, 1438, $1391,1300,1279,1249,1227,1112,1065,1041,926,899,764,747 \mathrm{~cm}^{-1}$; HRMS (APCI) m/z calculated for $\mathrm{C}_{15} \mathrm{H}_{14} \mathrm{NOSSe}[\mathrm{M}+\mathrm{H}]^{+}$: 335.9955, found 335.9959 .

(Z)-N-(2-Chlorophenyl)-3H-benzo[d][1,2]thiaselenol-3-imine (6m)<smiles>Clc1ccccc1/N=c1\osc2ccccc12</smiles>

Yield: $66 \%$ (108 mg). Yellow solid; $\mathrm{mp} 94-95{ }^{\circ} \mathrm{C} ;{ }^{1} \mathrm{H} \mathrm{NMR}\left(400 \mathrm{MHz}, \mathrm{CDCl}_{3}\right) \delta 8.26$ $(\mathrm{d}, J=8.0 \mathrm{~Hz}, 1 \mathrm{H}), 7.52-7.48(\mathrm{~m}, 3 \mathrm{H}), 7.34-7.28(\mathrm{~m}, 2 \mathrm{H}), 7.18-7.41(\mathrm{~m}, 2 \mathrm{H}) ;{ }^{13} \mathrm{C}$ $\operatorname{NMR}\left(101 \mathrm{MHz}, \mathrm{CDCl}_{3}\right) \delta 170.2,150.0,147.1,134.8,132.0,130.8,128.3,127.6$, $126.5,125.5,125.3,124.6,119.1$; IR (KBr) v 3048, 1622, 1577, 1561, 1470, 1434, 1270, 1258, 1241, $1191,1157,1056,1035,896,837,757,751 \mathrm{~cm}^{-1}$; HRMS (APCI) $\mathrm{m} / z$ calculated for $\mathrm{C}_{13} \mathrm{H}_{9} \mathrm{ClNSSe}[\mathrm{M}+$ $H]^{+}: 325.9303$, found 325.9307 . 
(Z)-N-(3,4-Dimethoxyphenyl)-3H-benzo[d][1,2]thiaselenol-3-imine (6n)

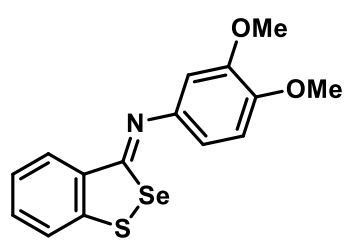

Yield: 71\% (125 mg). Yellow solid; mp 150-151 ${ }^{\circ} \mathrm{C} ;{ }^{1} \mathrm{H}$ NMR (400 MHz, $\mathrm{CDCl}_{3}$ ) $\delta 8.17(\mathrm{~d}, J=8.0 \mathrm{~Hz}, 1 \mathrm{H}), 7.47-7.41(\mathrm{~m}, 2 \mathrm{H}), 7.26-7.22(\mathrm{~m}, 1 \mathrm{H}), 6.91(\mathrm{~d}, J=$ 8.0 Hz, 1H), 6.77-6.75 (m, 2H), $3.91(\mathrm{~s}, 3 \mathrm{H}), 3.89(\mathrm{~s}, 3 \mathrm{H}) ;{ }^{13} \mathrm{C}$ NMR $(101 \mathrm{MHz}$, $\left.\mathrm{CDCl}_{3}\right) \delta 167.5,150.0,147.1,147.0,146.5,135.3,131.7,127.3,125.1,124.7,111.8,109.7,104.0$ $56.1,55.9 ; \operatorname{IR}(\mathrm{KBr})$ v 3047, 2953, 2833, 1614, 1596, 1578, 1502, 1459, 1435, 1294, 1268, 1247, 1188, $1107,1064,1038,894,830,757,752 \mathrm{~cm}^{-1}$; HRMS (APCI) $\mathrm{m} / z$ calculated for $\mathrm{C}_{15} \mathrm{H}_{14} \mathrm{NO}_{2} \mathrm{SSe}[\mathrm{M}+\mathrm{H}]^{+}$: 351.9905 , found 351.9904 .

(Z)-N-(3,4-Dichlorophenyl)-3H-benzo[d][1,2] thiaselenol-3-imine (6o)<smiles>Clc1ccc(/N=C2\Sc3ccccc32)cc1Cl</smiles>

Yield: 74\% (133 mg). Yellow solid; mp $113-114{ }^{\circ} \mathrm{C} ;{ }^{1} \mathrm{H}$ NMR (400 MHz, $\left.\mathrm{CDCl}_{3}\right) \delta$ $8.22(\mathrm{~d}, J=8.0 \mathrm{~Hz}, 1 \mathrm{H}), 7.59-7.50(\mathrm{~m}, 3 \mathrm{H}), 7.42-7.38(\mathrm{~m}, 1 \mathrm{H}), 7.30-7.26(\mathrm{~m}$,

$1 \mathrm{H}), 7.08(\mathrm{~d}, J=8.4 \mathrm{~Hz}, 1 \mathrm{H}) ;{ }^{13} \mathrm{C} N M R\left(101 \mathrm{MHz}, \mathrm{CDCl}_{3}\right) \delta 168.0,150.8,145.6$, $133.4,132.4,132.2,131.4,128.3,126.8,125.7,123.4,122.1,119.6$; $\mathrm{IR}(\mathrm{KBr}) v$ 3057, 1613, 1582, $1560,1482,1435,1309,1272,1259,1242,1187,1159,1084,1066,1008,900,824,753 \mathrm{~cm}^{-1}$; HRMS (APCI) $\mathrm{m} / z$ calculated for $\mathrm{C}_{13} \mathrm{H}_{8} \mathrm{Cl}_{2} \mathrm{NSSe}[\mathrm{M}+\mathrm{H}]^{+}: 359.8914$, found 359.8936 .

(Z)-N-(2,4-Dichlorophenyl)-3H-benzo[d][1,2] thiaselenol-3-imine (6p)<smiles>Clc1ccc(/N=c2\[cH-]sc3ccccc23)c(Cl)c1</smiles>

Yield: 65\% (117 mg). Yellow solid; mp $117-118^{\circ} \mathrm{C} ;{ }^{1} \mathrm{H}$ NMR (400 MHz, $\left.\mathrm{CDCl}_{3}\right) \delta$ 8.25-8.22 (m, 1H), 7.53-7.49 (m, 3H), 7.33-7.29 (m, 2H), 7.09 (d, J = 8.4 Hz, $1 \mathrm{H}) ;{ }^{13} \mathrm{CNMR}\left(101 \mathrm{MHz}, \mathrm{CDCl}_{3}\right) \delta 171.0,148.5,147.2,134.7,132.2,131.0$, 130.6, 128.5, 127.6, 126.5, 125.4, 124.6, 120.0; IR (KBr) v 3056, 1619, 1605, 1582, 1574, 1470, 1434, 
1384, 155, 1190, 1140, 1100, 1058, 958, 895, 867, 813, $754 \mathrm{~cm}^{-1}$; HRMS (APCI) m/z calculated for $\mathrm{C}_{13} \mathrm{H}_{8} \mathrm{Cl}_{2} \mathrm{NSSe}[\mathrm{M}+\mathrm{H}]^{+}: 359.8914$, found 359.8939 .

\section{(Z)-N-(Pyridin-2-yl)-3H-benzo[d][1,2]thiaselenol-3-imine (6q)}

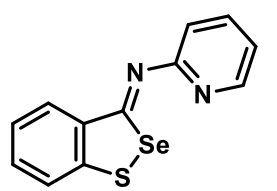

Yield: $74 \%$ (108 mg). Yellow solid; $\mathrm{mp} 121-122{ }^{\circ} \mathrm{C} ;{ }^{1} \mathrm{H}$ NMR $\left(400 \mathrm{MHz}, \mathrm{CDCl}_{3}\right) \delta$ $8.59(\mathrm{~d}, J=4.8 \mathrm{~Hz}, 1 \mathrm{H}), 8.53(\mathrm{~d}, J=8.4 \mathrm{~Hz}, 1 \mathrm{H}), 7.85-7.81(\mathrm{~m}, 1 \mathrm{H}), 7.66(\mathrm{~d}, J=8.0$ $\mathrm{Hz}, 1 \mathrm{H}), 7.58-7.51(\mathrm{~m}, 2 \mathrm{H}), 7.37(\mathrm{t}, J=7.2 \mathrm{~Hz}, 1 \mathrm{H}), 7.15(\mathrm{t}, J=6.0 \mathrm{~Hz}, 1 \mathrm{H}) ;{ }^{13} \mathrm{C} \mathrm{NMR}\left(101 \mathrm{MHz}, \mathrm{CDCl}_{3}\right)$ $\delta 170.0,156.5,146.8,142.5,138.0,134.8,131.3,127.2,124.4,123.8,121.2,119.4 ; \mathrm{IR}(\mathrm{KBr}) v 3063$ $3047,1588,1561,1505,1460,1433,1316,1263,1207,1141,1073,998,946,864,784,754,738$ $720 \mathrm{~cm}^{-1}$; HRMS (APCI) m/z calculated for $\mathrm{C}_{12} \mathrm{H}_{9} \mathrm{~N}_{2} \mathrm{SSe}[\mathrm{M}+\mathrm{H}]^{+}: 292.9646$, found 292.9658 .

\section{(Z)-5-Methyl-N-phenyl-3H-benzo[d][1,2]thiaselenol-3-imine (6r)}<smiles></smiles>

Yield: 73\% (112 mg). Yellow solid; mp $86-87{ }^{\circ} \mathrm{C} ;{ }^{1} \mathrm{H} \mathrm{NMR}\left(400 \mathrm{MHz}, \mathrm{CDCl}_{3}\right) \delta$ 8.04-7.99 (m, 1H), 7.47-7.42 (m, 2H), $7.36(\mathrm{~d}, J=8.4 \mathrm{~Hz}, 1 \mathrm{H}), 7.30-7.28(\mathrm{~m}$, $1 \mathrm{H}), 7.25-7.21(\mathrm{~m}, 1 \mathrm{H}), 7.15-7.12(\mathrm{~m}, 2 \mathrm{H}), 2.44(\mathrm{~s}, 3 \mathrm{H}) ;{ }^{13} \mathrm{C} \mathrm{NMR}\left(101 \mathrm{MHz}, \mathrm{CDCl}_{3}\right) \delta 168.5,153.4$, $143.7,135.2,135.2,133.1,130.0,127.2,125.7,124.2,119.0,20.6$; IR (KBr) v 3048, 2916, 1607, 1581, $1482,1466,1395,1380,1313,1266,1243,1201,1154,1132,1068,955,838,800,754,695 \mathrm{~cm}^{-1}$; HRMS (APCI) $m / z$ calculated for $\mathrm{C}_{14} \mathrm{H}_{12} \mathrm{NSSe}[\mathrm{M}+\mathrm{H}]^{+}: 305.9850$, found 305.9879 . 
(Z)-5-Methyl-N-(p-tolyl)-3H-benzo[d][1,2]thiaselenol-3-imine (6s)

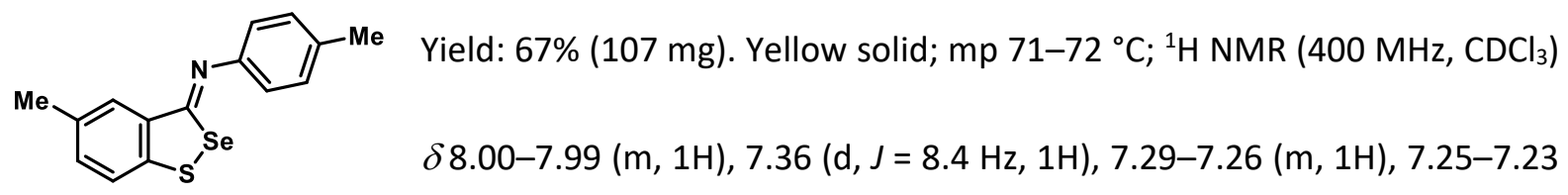

(m, 2H), 7.05-7.03 (m, 2H), $2.43(\mathrm{~s}, 3 \mathrm{H}), 2.39(\mathrm{~s}, 3 \mathrm{H}) ;{ }^{13} \mathrm{C} \mathrm{NMR}\left(101 \mathrm{MHz}, \mathrm{CDCl}_{3}\right) \delta 168.0,150.9,143.6$, $135.4,135.2,135.2,133.0,130.6,127.2,124.2,118.8,21.1,20.6$; IR $(\mathrm{KBr}) v 3056,3020,2919,2854$ 1620, 1592, 1503, 1466, 1395, 1316, 1264, 1242, 1211, 1155, 1107, 1066, 952, 852, 823, $807 \mathrm{~cm}^{-1}$; HRMS (APCl) $m / z$ calculated for $\mathrm{C}_{15} \mathrm{H}_{14} \mathrm{NSSe}[\mathrm{M}+\mathrm{H}]^{+}: 320.0006$, found 320.0024 .

(Z)-N-(4-Chlorophenyl)-5-methyl-3H-benzo[d][1,2]thiaselenol-3-imine (6t)

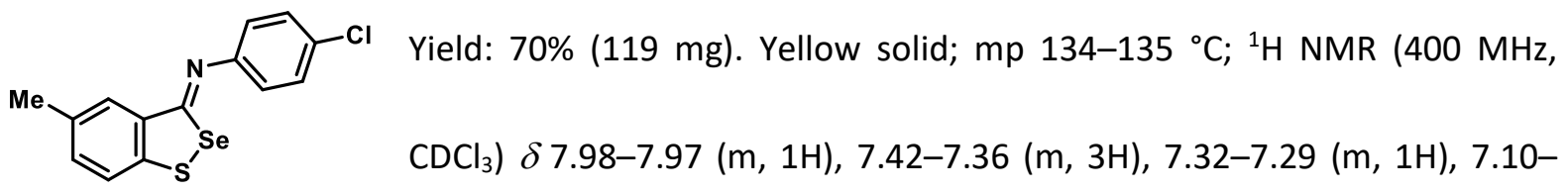
$7.06(\mathrm{~m}, 2 \mathrm{H}), 2.44(\mathrm{~s}, 3 \mathrm{H}) ;{ }^{13} \mathrm{C} \mathrm{NMR}\left(101 \mathrm{MHz}, \mathrm{CDCl}_{3}\right) \delta 169.2,151.7,143.8,135.3,135.1,133.3,130.8$, $130.2,127.2,124.2,120.5,20.6 ; \mathrm{IR}(\mathrm{KBr})$ v 3017, 2922, 1613, 1596, 1582, 1481, 1468, 1395, 1318, $1265,1243,1205,1156,1134,1086,1066,1006,954,849,798 \mathrm{~cm}^{-1}$; HRMS (APCI) $\mathrm{m} / \mathrm{z}$ calculated for $\mathrm{C}_{14} \mathrm{H}_{11} \mathrm{ClNSSe}[\mathrm{M}+\mathrm{H}]^{+}:$339.9460, found 339.9475 .

(Z)-5-Methoxy-N-phenyl-3H-benzo[d][1,2]thiaselenol-3-imine (6u)<smiles>COc1ccc2s[se]c(=Nc3ccccc3)c2c1</smiles>
(m, 1H), 7.15-7.08 (m, 3H), $\left.3.88(\mathrm{~s}, 3 \mathrm{H}) ;{ }^{13} \mathrm{C} \mathrm{NMR} \mathrm{(101} \mathrm{MHz,} \mathrm{CDCl}_{3}\right) \delta 168.1,157.8,153.3,138.3$ $136.4,130.1,125.7,124.8,121.6,119.0,108.6,55.7$; IR (KBr) v 3058, 3016, 2965, 2934, 2829, 1614, 1589, 1471, 1434, 1401, 1431, 1267, 1217, 1191, 1022, 973, 859, 807, 783, $691 \mathrm{~cm}^{-1}$; HRMS (APCI) 
$m / z$ calculated for $\mathrm{C}_{14} \mathrm{H}_{12} \mathrm{NOSSe}[\mathrm{M}+\mathrm{H}]^{+}: 321.9799$, found 321.9813 .

(Z)-5-Methoxy- $N$-(p-tolyl)-3H-benzo[d][1,2]thiaselenol-3-imine (6v)

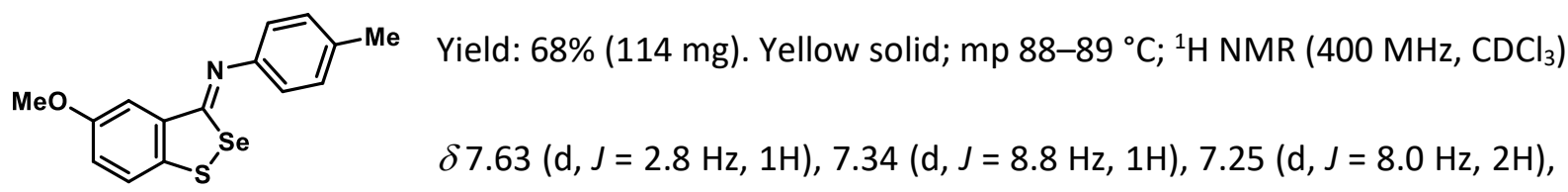

$7.10(\mathrm{dd}, J=8.8,2.8 \mathrm{~Hz}, 1 \mathrm{H}), 7.06-7.04(\mathrm{~m}, 2 \mathrm{H}), 3.89(\mathrm{~s}, 3 \mathrm{H}), 2.39(\mathrm{~s}, 3 \mathrm{H}) ;{ }^{13} \mathrm{C} \mathrm{NMR}\left(101 \mathrm{MHz}, \mathrm{CDCl}_{3}\right) \delta$ $167.6,157.7,150.8,138.2,136.5,135.5,130.6,124.8,121.5,118.8,108.6,55.8,21.1 ; \mathrm{IR}(\mathrm{KBr})$ v 3054, $3001,2954,2823,1596,1504,1469,1401,1342,1265,1216,1184,1156,1121,1106,1028,973$, 848, 807, $660 \mathrm{~cm}^{-1}$; HRMS (APCl) $\mathrm{m} / \mathrm{z}$ calculated for $\mathrm{C}_{15} \mathrm{H}_{14} \mathrm{NOSSe}[\mathrm{M}+\mathrm{H}]^{+}: 335.9955$, found 335.9959.

(Z)-N-(4-Chlorophenyl)-5-methoxy-3H-benzo[d][1,2]thiaselenol-3-imine (6w)

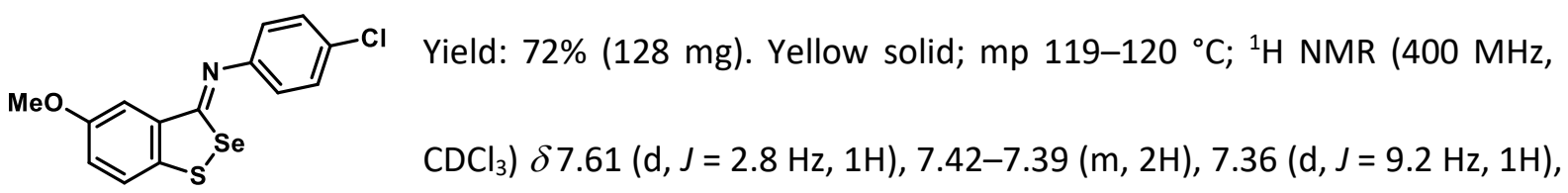
7.14-7.08 (m, 3H), 3.89 (s, 3H); $\left.{ }^{13} \mathrm{C} \mathrm{NMR} \mathrm{(101} \mathrm{MHz,} \mathrm{CDCl}_{3}\right) \delta 168.8,157.8,151.6,138.4,136.3,130.9$, $130.2,124.8,121.7,120.5,108.6,55.8$; IR (KBr) v 3062, 2927, 2849, 1611, 1596, 1583, 1484, 1471, $1404,1342,1267,1222,1162,1092,1027,977,849,812,661 \mathrm{~cm}^{-1}$; HRMS (APCI) $\mathrm{m} / z$ calculated for $\mathrm{C}_{14} \mathrm{H}_{11} \mathrm{ClNOSSe}[\mathrm{M}+\mathrm{H}]^{+}: 355.9409$, found 355.9437. 
(Z)-5-Fluoro- $N$-( $p$-tolyl)-3H-benzo[d][1,2] thiaselenol-3-imine (6x)

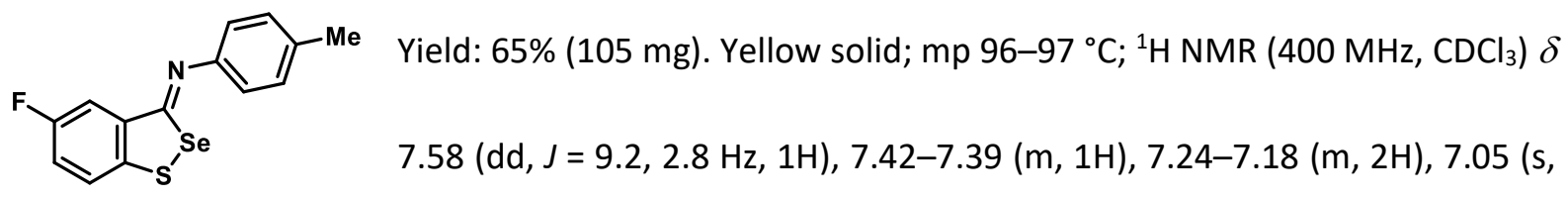

1H), $7.03(\mathrm{~s}, 1 \mathrm{H}), 2.38(\mathrm{~s}, 3 \mathrm{H}) ;{ }^{13} \mathrm{C}$ NMR $\left(101 \mathrm{MHz}, \mathrm{CDCl}_{3}\right) \delta 165.5\left(\mathrm{~d}, J_{\mathrm{C}-\mathrm{F}}=2.9 \mathrm{~Hz}\right), 160.7\left(\mathrm{~d}, \mathrm{~J}_{\mathrm{C}-\mathrm{F}}=\right.$ $244.6 \mathrm{~Hz}), 150.4,141.2\left(\mathrm{~d}, J_{\mathrm{C}-\mathrm{F}}=1.2 \mathrm{~Hz}\right), 137.5\left(\mathrm{~d}, J_{\mathrm{C}-\mathrm{F}}=7.7 \mathrm{~Hz}\right), 135.8,130.6,125.0\left(\mathrm{~d}, J_{\mathrm{C}-\mathrm{F}}=8.1 \mathrm{~Hz}\right)$, $119.4\left(\mathrm{~d}, J_{\mathrm{C}-\mathrm{F}}=24.6 \mathrm{~Hz}\right), 118.7,113.1\left(\mathrm{~d}, J_{\mathrm{C}-\mathrm{F}}=23.9 \mathrm{~Hz}\right), 21.1$; IR $(\mathrm{KBr}) \vee 3065,3026,2916,2857,1616$, $1593,1503,1463,1407,1318,1257,1197,1108,1067,982,882,876,855,826,711 \mathrm{~cm}^{-1}$; HRMS (APCI) $m / z$ calculated for $\mathrm{C}_{14} \mathrm{H}_{11} \mathrm{FNSSe}[\mathrm{M}+\mathrm{H}]^{+}: 323.9755$, found 323.9758 .

(Z)-N-(4-Chlorophenyl)-5-fluoro-3H-benzo[d][1,2] thiaselenol-3-imine (6y)

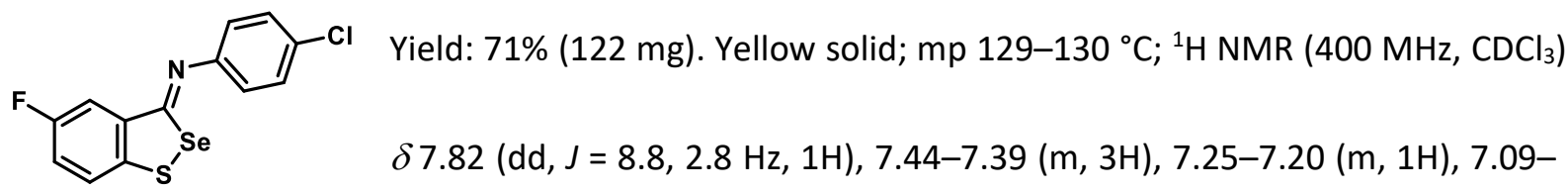

$7.05(\mathrm{~m}, 2 \mathrm{H}) ;{ }^{13} \mathrm{C} N M R\left(101 \mathrm{MHz}, \mathrm{CDCl}_{3}\right) \delta 167.0\left(\mathrm{~d}, \mathrm{~J}_{\mathrm{C}-\mathrm{F}}=3.0 \mathrm{~Hz}\right), 160.7\left(\mathrm{~d}, J_{\mathrm{C}-\mathrm{F}}=245.1 \mathrm{~Hz}\right), 151.2$ $141.5\left(\mathrm{~d}, J_{\mathrm{C}-\mathrm{F}}=1.3 \mathrm{~Hz}\right), 137.3\left(\mathrm{~d}, J_{\mathrm{C}-\mathrm{F}}=7.8 \mathrm{~Hz}\right), 131.1,130.2,125.1\left(\mathrm{~d}, J_{\mathrm{C}-\mathrm{F}}=8.0 \mathrm{~Hz}\right), 120.4,119.8\left(\mathrm{~d}, J_{\mathrm{C}-}\right.$ $\mathrm{F}=24.4 \mathrm{~Hz}), 113.1\left(\mathrm{~d}, J_{\mathrm{C}-\mathrm{F}}=24.0 \mathrm{~Hz}\right) ; \mathrm{IR}(\mathrm{KBr}) v 3062,1601,1583,1483,1464,1407,1323,1241,1194$, 1087, 1063, 1009, 980, 883, 854, 813, 787, $711 \mathrm{~cm}^{-1}$; HRMS (APCI) $\mathrm{m} / \mathrm{z}$ calculated for $\mathrm{C}_{13} \mathrm{H}_{8} \mathrm{ClFNSSe}$ $[M+H]^{+}: 343.9209$, found 343.9207 . 


\section{(Z)-5-Chloro-N-phenyl-3H-benzo[d][1,2] thiaselenol-3-imine (6z)}

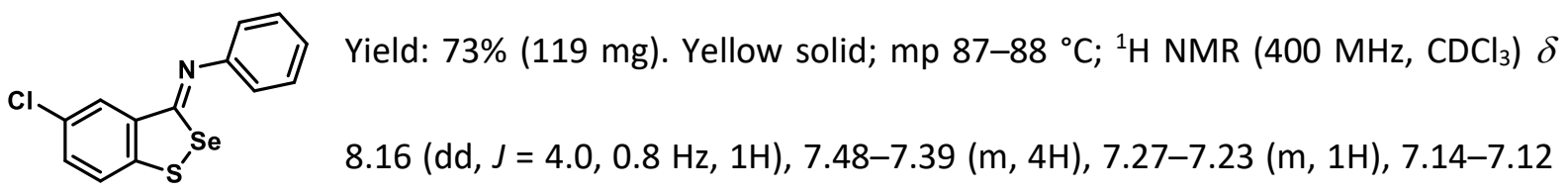

(m, 2H); ${ }^{13} \mathrm{C}$ NMR $\left(101 \mathrm{MHz}, \mathrm{CDCl}_{3}\right) \delta 166.1,152.9,144.7,137.0,131.6,131.4,130.1,126.8,126.0$ $125.1,118.8$; IR (KBr) v 3059, 1614, 1587, 1575, 1483, 1452, 1387, 1307, 1239, 1165, 1084, 1022, 958, 884, 837, 818, 763, $692 \mathrm{~cm}^{-1}$; HRMS (APCI) $\mathrm{m} / \mathrm{z}$ calculated for $\mathrm{C}_{13} \mathrm{H}_{9} \mathrm{ClNSSe}[\mathrm{M}+\mathrm{H}]^{+}: 325.9303$, found 325.9305 .

(Z)-5-Chloro-N-(p-tolyl)-3H-benzo[d][1,2]thiaselenol-3-imine (6aa)

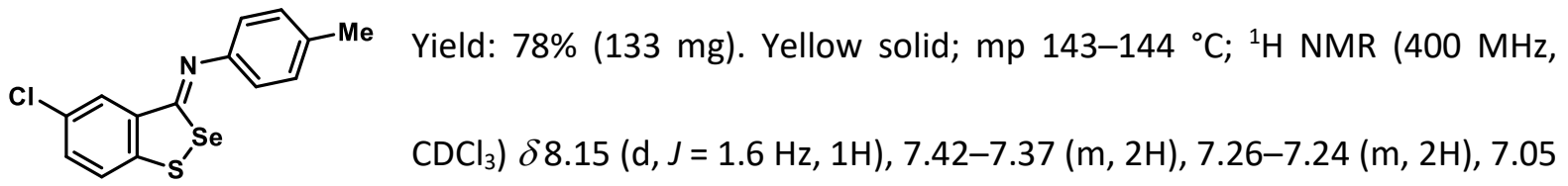

(s, 1H), $7.03(\mathrm{~s}, 1 \mathrm{H}), 2.39(\mathrm{~s}, 3 \mathrm{H}) ;{ }^{13} \mathrm{C}$ NMR $\left(101 \mathrm{MHz}, \mathrm{CDCl}_{3}\right) \delta 165.4,150.4,144.6,137.2,135.8,131.4$, $131.3,130.6,126.8,125.1,118.7,21.1 ; \mathrm{IR}(\mathrm{KBr})$ v 3056, 2921, 2851, 1604, 1577, 1485, 1450, 1389, 1309, 1243, 1175, 1162, 1085, 1010, 945, 890, 828, 811, 749, $678 \mathrm{~cm}^{-1}$; HRMS (APCI) $\mathrm{m} / \mathrm{z}$ calculated for $\mathrm{C}_{14} \mathrm{H}_{11} \mathrm{CINSSe}[\mathrm{M}+\mathrm{H}]^{+}: 339.9460$, found 339.9475 .

(Z)-5-Chloro-N-(4-chlorophenyl)-3H-benzo[d][1,2]thiaselenol-3-imine (6ab)

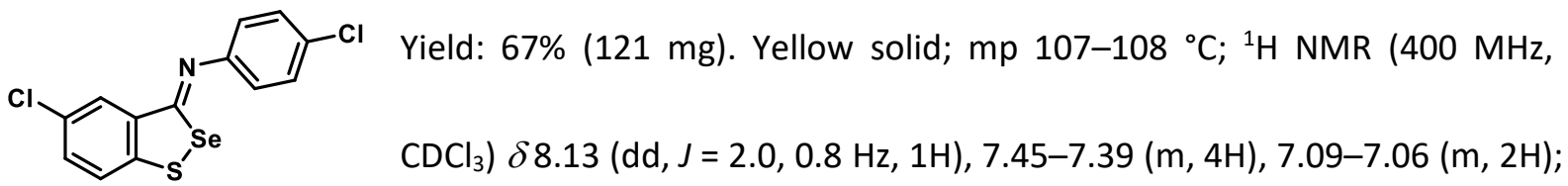

${ }^{13} \mathrm{C}$ NMR $\left(101 \mathrm{MHz}, \mathrm{CDCl}_{3}\right) \delta 166.9,151.2,144.8,136.9,131.7,131.5,131.2,130.2,126.8,125.1$, 120.4; IR (KBr) v 3061, 3020, 1615, 1599, 1574, 1501, 1450, 1387, 1308, 1238, 1177, 1165, 1107, 1083, 883, 850, 814, $774 \mathrm{~cm}^{-1}$; HRMS (APCl) $\mathrm{m} / z$ calculated for $\mathrm{C}_{13} \mathrm{H}_{8} \mathrm{Cl}_{2} \mathrm{NSSe}[\mathrm{M}+\mathrm{H}]^{+}: 359.8914$, 
found 359.8936 .

(Z)-7-Methyl-N-(p-tolyl)-3H-benzo[d][1,2]thiaselenol-3-imine (6ac)

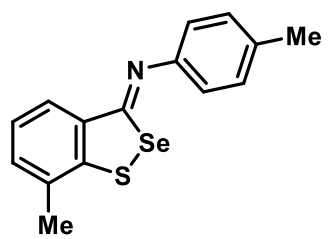

Yield: $76 \%$ (122 mg). Yellow solid; mp 90-91 ${ }^{\circ} \mathrm{C} ;{ }^{1} \mathrm{H}$ NMR (400 MHz, $\left.\mathrm{CDCl}_{3}\right) \delta$ $8.05(\mathrm{dd}, J=8.0,0.8 \mathrm{~Hz}, 1 \mathrm{H}), 7.29(\mathrm{dd}, J=6.8,0.8 \mathrm{~Hz}, 1 \mathrm{H}), 7.25-7.21(\mathrm{~m}, 3 \mathrm{H})$, 7.05-7.03 (m, 2H), $2.46(\mathrm{~s}, 3 \mathrm{H}), 2.38(\mathrm{~s}, 3 \mathrm{H}) ;{ }^{13} \mathrm{C} \mathrm{NMR}\left(101 \mathrm{MHz}, \mathrm{CDCl}_{3}\right) \delta 168.1$, $150.9,146.6,135.4,135.1,133.9,132.1,130.6,125.5,125.2,118.9,21.1,19.7 ; \mathrm{IR}(\mathrm{KBr}) v$ 3066, 3026, $2932,1603,1520,1448,1404,1379,1307,1250,1200,1102,1048,1019,957,862,830,776,712$, $689 \mathrm{~cm}^{-1}$; HRMS (APCI) $\mathrm{m} / z$ calculated for $\mathrm{C}_{15} \mathrm{H}_{14} \mathrm{NSSe}[\mathrm{M}+\mathrm{H}]^{+}: 320.0006$, found 320.0025 .

(Z)-N-(4-Chlorophenyl)-7-methyl-3H-benzo[d][1,2]thiaselenol-3-imine (6ad)<smiles>O=[N+]([O-])c1cccc2c(=Nc3ccc(Cl)cc3)[se][se]c12</smiles>

Yield: 56\% (95 mg). Yellow solid; mp 102-103 ${ }^{\circ} \mathrm{C} ;{ }^{1} \mathrm{H} \mathrm{NMR}\left(400 \mathrm{MHz}, \mathrm{CDCl}_{3}\right) \delta$ $8.03(\mathrm{dd}, J=8.0,0.8 \mathrm{~Hz}, 1 \mathrm{H}), 7.42-7.39(\mathrm{~m}, 2 \mathrm{H}), 7.33-7.31(\mathrm{~m}, 1 \mathrm{H}), 7.26-7.22$ (m, 1H), 7.10-7.06 (m, 2H), $2.47(\mathrm{~s}, 3 \mathrm{H}) ;{ }^{13} \mathrm{C} \mathrm{NMR}\left(101 \mathrm{MHz}, \mathrm{CDCl}_{3}\right) \delta 169.4$, $151.7,146.8,135.0,134.0,132.3,130.8,130.1,125.6,125.2,120.6,19.7 ; \mathrm{IR}(\mathrm{KBr}) v 3052,2968,2923$, $2850,1606,1579,1481,1450,1404,1305,1250,1199,1164,1087,1050,1008,956,859,825,786$ $\mathrm{cm}^{-1}$; HRMS (APCI) $\mathrm{m} / \mathrm{z}$ calculated for $\mathrm{C}_{14} \mathrm{H}_{11} \mathrm{CINSSe}[\mathrm{M}+\mathrm{H}]^{+}: 339.9460$, found 339.9475 . 
$(6 Z, 12 Z)-N^{6}, N^{12}$-Diphenyl-6H,12H-dibenzo[b,f][1,5]dithiocine-6,12-diimine (7a)

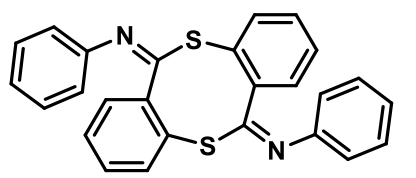

Yield: 79\% (190 mg). White solid; mp $171-172{ }^{\circ} \mathrm{C} ;{ }^{1} \mathrm{H}$ NMR $(400 \mathrm{MHz}$, $\left.\mathrm{CDCl}_{3}\right) \delta 7.50(\mathrm{t}, J=8.0 \mathrm{~Hz}, 4 \mathrm{H}), 7.36(\mathrm{dd}, J=7.6,1.2 \mathrm{~Hz}, 2 \mathrm{H}), 7.30-7.27(\mathrm{~m}$, $2 \mathrm{H}), 7.26-7.22(\mathrm{~m}, 4 \mathrm{H}), 7.20-7.18(\mathrm{~m}, 4 \mathrm{H}), 7.14-7.10(\mathrm{~m}, 2 \mathrm{H}) ;{ }^{13} \mathrm{C} \mathrm{NMR}\left(101 \mathrm{MHz}, \mathrm{CDCl}_{3}\right) \delta 166.6$, $149.5,143.2,136.0,130.5,129.8,129.3,128.6,128.5,125.0,120.2 ; \mathrm{IR}(\mathrm{KBr}) v$ 3059, 3028, 1586, 1482, 1431, 1271, 1236, 1188, 1164, 1072, 1061, 1023, 951, 923, 827, 768, 714, 722, $695 \mathrm{~cm}^{-1}$; HRMS (APCl) $m / z$ calculated for $\mathrm{C}_{26} \mathrm{H}_{19} \mathrm{~N}_{2} \mathrm{~S}_{2}[\mathrm{M}+\mathrm{H}]^{+}: 423.0984$, found 423.0978 .

$(6 Z, 12 Z)-N^{6}, N^{12}$-Di-p-tolyl-6H,12H-dibenzo[b,f][1,5]dithiocine-6,12-diimine (7b)<smiles>Cc1ccc(/N=C2\Sc3ccccc3/C(=N\c3ccc(C)cc3)Sc3ccccc32)cc1</smiles>

Yield: $83 \%$ (187 mg). White solid; mp $225-226{ }^{\circ} \mathrm{C} ;{ }^{1} \mathrm{H}$ NMR (400 $\left.\mathrm{MHz}, \mathrm{CDCl}_{3}\right) \delta 7.35(\mathrm{dd}, J=7.6,1.6 \mathrm{~Hz}, 2 \mathrm{H}), 7.31-7.28(\mathrm{~m}, 4 \mathrm{H})$, 7.27-7.25 (m, 2H), 7.22 (dd, J = 7.6, $0.8 \mathrm{~Hz}, 2 \mathrm{H}), 7.13-7.09(\mathrm{~m}, 6 \mathrm{H}), 2.41(\mathrm{~s}, 6 \mathrm{H}) ;{ }^{13} \mathrm{C}$ NMR $(101 \mathrm{MHz}$, $\left.\mathrm{CDCl}_{3}\right) \delta 166.3,147.0,143.3,136.0,134.6,130.5,129.9,129.7,128.6,128.6,120.1,21.1 ; \mathrm{IR}(\mathrm{KBr}) v$ $3057,3018,2975,2916,1622,1591,1482,1433,1271,1202,1178,1105,1060,1026,945,928,766$, 717, 691, $638 \mathrm{~cm}^{-1}$; HRMS (APCI) $\mathrm{m} / z$ calculated for $\mathrm{C}_{28} \mathrm{H}_{23} \mathrm{~N}_{2} \mathrm{~S}_{2}[\mathrm{M}+\mathrm{H}]^{+}:$: 451.1297, found 451.1322 .

$(6 Z, 12 Z)-N^{6}, N^{12}$-Bis(4-methoxyphenyl)-6H,12H-dibenzo[b,f][1,5]dithiocine-6,12-diimine (7c)<smiles>COc1ccc(/N=C2\Sc3ccccc3/C(=N\c3ccc(OC)cc3)Sc3ccccc32)cc1</smiles>
Yield: 80\% (193 mg). White solid; mp $224-225{ }^{\circ} \mathrm{C} ;{ }^{1} \mathrm{H}$ NMR (400 MHz, $\left.\mathrm{CDCl}_{3}\right) \delta 7.36-7.34(\mathrm{~m}, 2 \mathrm{H}), 7.29-7.25(\mathrm{~m}, 2 \mathrm{H}), 7.22$ $(\mathrm{dd}, J=7.6,0.8 \mathrm{~Hz}, 2 \mathrm{H}), 7.20-7.16(\mathrm{~m}, 4 \mathrm{H}), 7.13-7.09(\mathrm{~m}, 2 \mathrm{H}), 7.05-7.01(\mathrm{~m}, 4 \mathrm{H}), 3.87(\mathrm{~s}, 6 \mathrm{H}) ;{ }^{13} \mathrm{C}$ $\operatorname{NMR}\left(101 \mathrm{MHz}, \mathrm{CDCl}_{3}\right) \delta 166.0,157.1,143.4,142.5,136.0,130.5,129.7,128.7,121.8,114.5,55.5 ; \mathrm{IR}$ $(\mathrm{KBr})$ v 3059, 2965, 2908, 2832, 1613, 1577, 1499, 1462, 1437, 1293, 1235, 1185, 1100, 1062, 1025, 
954, 943, 925, 833, 755, $710 \mathrm{~cm}^{-1}$; HRMS (APCI) $\mathrm{m} / \mathrm{z}$ calculated for $\mathrm{C}_{28} \mathrm{H}_{23} \mathrm{~N}_{2} \mathrm{O}_{2} \mathrm{~S}_{2}[\mathrm{M}+\mathrm{H}]^{+}:$483.1195, found 483.1192 .

$(6 Z, 12 Z)-N^{6}, N^{12}$-Bis(4-fluorophenyl)-6H,12H-dibenzo[b,f][1,5]dithiocine-6,12-diimine (7d)<smiles>Fc1ccc(/N=c2/sc3ccccc3/c(=N/c3ccc(F)cc3)c3ccccc23)cc1</smiles>

Yield: $81 \%$ (186 mg). White solid; $\mathrm{mp} 150-151{ }^{\circ} \mathrm{C} ;{ }^{1} \mathrm{H}$ NMR (400

$\left.\mathrm{MHz}, \mathrm{CDCl}_{3}\right) \delta 7.36-7.33(\mathrm{~m}, 2 \mathrm{H}), 7.31-7.27(\mathrm{~m}, 2 \mathrm{H}), 7.26-7.11(\mathrm{~m}$, $12 \mathrm{H}) ;{ }^{13} \mathrm{C}$ NMR $\left(101 \mathrm{MHz}, \mathrm{CDCl}_{3}\right) \delta 167.1,160.3\left(\mathrm{~d}, J_{\mathrm{C}-\mathrm{F}}=242.2 \mathrm{~Hz}\right), 145.4\left(\mathrm{~d}, J_{\mathrm{C}-\mathrm{F}}=2.9 \mathrm{~Hz}\right), 143.1$, $136.1,130.7,129.9,128.6,128.2,121.7\left(\mathrm{~d}, J_{C-F}=8.1 \mathrm{~Hz}\right), 116.1\left(\mathrm{~d}, J_{\mathrm{C}-\mathrm{F}}=22.5 \mathrm{~Hz}\right) ; \mathrm{IR}(\mathrm{KBr}) v 3054$, $1609,1498,1463,1432,1272,1216,1183,1150,1092,1061,1009,944,930,877,834,782,758$ $712,642 \mathrm{~cm}^{-1}$; HRMS (APCl) $\mathrm{m} / z$ calculated for $\mathrm{C}_{26} \mathrm{H}_{17} \mathrm{~F}_{2} \mathrm{~N}_{2} \mathrm{~S}_{2}[\mathrm{M}+\mathrm{H}]^{+}:$459.0796, found 459.0816 .

$(6 Z, 12 Z)-N^{6}, N^{12}$-Bis(4-chlorophenyl)-6H,12H-dibenzo[b,f][1,5]dithiocine-6,12-diimine (7e)

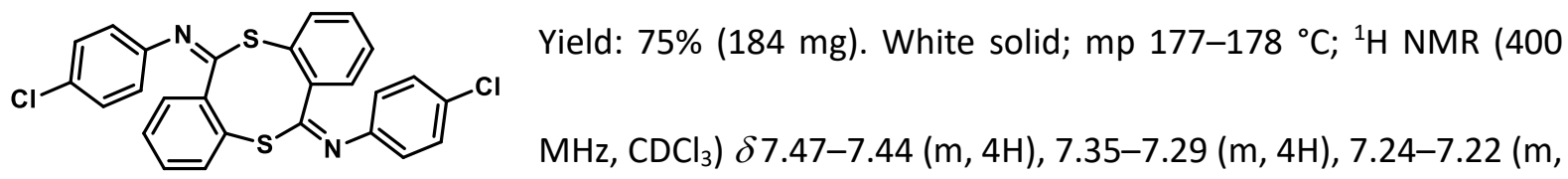
2H), $7.15(\mathrm{dd}, J=7.2,1.6 \mathrm{~Hz}, 2 \mathrm{H}), 7.13-7.09(\mathrm{~m}, 4 \mathrm{H}) ;{ }^{13} \mathrm{C} \mathrm{NMR}\left(101 \mathrm{MHz}, \mathrm{CDCl}_{3}\right) \delta 167.3,147.9,143.0$, $136.1,130.8,130.4,130.0,129.5,128.5,128.1,121.5 ; \mathrm{IR}(\mathrm{KBr})$ v 3060, 1617, 1582, 1482, 1433, 1398, 1273, 1236, 1187, 1164, 1086, 1059, 1011, 945, 932, 832, 761, 720, $668 \mathrm{~cm}^{-1}$; HRMS (APCI) $\mathrm{m} / \mathrm{z}$ calculated for $\mathrm{C}_{26} \mathrm{H}_{17} \mathrm{Cl}_{2} \mathrm{~N}_{2} \mathrm{~S}_{2}[\mathrm{M}+\mathrm{H}]^{+}:$491.0205, found 491.0206. 
$(6 Z, 12 Z)-N^{6}, N^{12}$-Di-m-tolyl-6H,12H-dibenzo[b,f][1,5]dithiocine-6,12-diimine (7f)

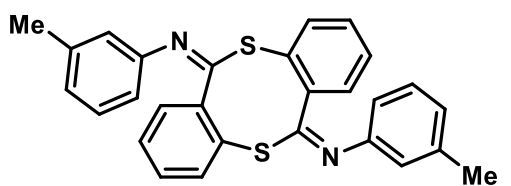

Yield: $78 \%$ (176 mg). White solid; mp $131-132{ }^{\circ} \mathrm{C} ;{ }^{1} \mathrm{H}$ NMR (400 $\left.\mathrm{MHz}, \mathrm{CDCl}_{3}\right) \delta 7.40-7.33(\mathrm{~m}, 4 \mathrm{H}), 7.29-7.27(\mathrm{~m}, 2 \mathrm{H}), 7.23(\mathrm{~d}, J=$ $7.6 \mathrm{~Hz}, 2 \mathrm{H}), 7.13-7.09(\mathrm{~m}, 2 \mathrm{H}), 7.07-7.00(\mathrm{~m}, 6 \mathrm{H}), 2.47(\mathrm{~s}, 6 \mathrm{H}) ;{ }^{13} \mathrm{C} \mathrm{NMR}\left(101 \mathrm{MHz}, \mathrm{CDCl}_{3}\right) \delta 166.5$, 149.6, 143.2, 139.2, 136.0, 130.5, 129.7, 129.1, 128.6, 128.4, 125.8, 120.7, 117.1, 21.6; IR (KBr) $v$ $3057,2961,2918,2854,1629,1598,1581,1479,1462,1432,1276,1257,1212,1144,1059,950$, 915, 784, 770, $710 \mathrm{~cm}^{-1}$; HRMS (APCl) $\mathrm{m} / \mathrm{z}$ calculated for $\mathrm{C}_{28} \mathrm{H}_{23} \mathrm{~N}_{2} \mathrm{~S}_{2}[\mathrm{M}+\mathrm{H}]^{+}:$451.1297, found 451.1325 .

$(6 Z, 12 Z)-N^{6}, N^{12}$-Di-o-tolyl-6H,12H-dibenzo[b,f][1,5]dithiocine-6,12-diimine (7g)<smiles>Cc1ccccc1/N=C1\Sc2ccccc2S/C(=N/c2ccccc2C)c2ccccc21</smiles>
Yield: $81 \%$ (183 mg). White solid; mp $195-196{ }^{\circ} \mathrm{C} ;{ }^{1} \mathrm{H}$ NMR $(400 \mathrm{MHz}$, $\left.\mathrm{CDCl}_{3}\right) \delta 7.37(\mathrm{dd}, J=7.6,1.6 \mathrm{~Hz}, 2 \mathrm{H}), 7.33-7.29(\mathrm{~m}, 6 \mathrm{H}), 7.27-7.23(\mathrm{~m}$, $2 \mathrm{H}), 7.17-7.11(\mathrm{~m}, 4 \mathrm{H}), 7.03(\mathrm{~d}, J=7.6 \mathrm{~Hz}, 2 \mathrm{H}), 2.36(\mathrm{~s}, 6 \mathrm{H}) ;{ }^{13} \mathrm{C}$ NMR $(101$ $\left.\mathrm{MHz}, \mathrm{CDCl}_{3}\right) \delta 166.4,148.3,143.1,136.2,130.8,130.6,129.8,128.6,128.6,127.9,126.7,125.0$ 119.1, 17.8; IR (KBr) v 3067, 3018, 2975, 2943, 2916, 1637, 1623, 1596, 1483, 1458, 1430, 1271, 1203, 1178, 1108, 954, 839, 766,719, $651 \mathrm{~cm}^{-1}$; HRMS (APCI) $\mathrm{m} / \mathrm{z}$ calculated for $\mathrm{C}_{28} \mathrm{H}_{23} \mathrm{~N}_{2} \mathrm{~S}_{2}[\mathrm{M}+\mathrm{H}]^{+}$: 451.1297, found 451.1324 .

$(6 Z, 12 Z)-N^{6}, N^{12}$-Bis(3-chlorophenyl)-6H,12H-dibenzo[b,f][1,5]dithiocine-6,12-diimine (7h)<smiles>Clc1cccc(/N=c2/sc3ccccc3/c(=N/c3cccc(Cl)c3)c3ccccc23)c1</smiles>
Yield: $75 \%$ (184 mg). White solid; mp $137-138{ }^{\circ} \mathrm{C}$; ${ }^{1} \mathrm{H}$ NMR (400 $\left.\mathrm{MHz}, \mathrm{CDCl}_{3}\right) \delta 7.44(\mathrm{t}, J=8.0 \mathrm{~Hz}, 2 \mathrm{H}), 7.35-7.28(\mathrm{~m}, 4 \mathrm{H}), 7.25-7.21$ $(\mathrm{m}, 4 \mathrm{H}), 7.18-7.13(\mathrm{~m}, 4 \mathrm{H}), 7.08-7.05(\mathrm{~m}, 2 \mathrm{H}) ;{ }^{13} \mathrm{C} \mathrm{NMR}\left(101 \mathrm{MHz}, \mathrm{CDCl}_{3}\right) \delta 167.9,150.7,142.8$ 
$136.0,134.9,130.8,130.6,130.0,128.3,128.0,125.0,120.3,118.3 ; \mathrm{IR}(\mathrm{KBr}) v$ 3058, 1620, 1585, $1556,1467,1433,1270,1235,1183,1159,1089,1069,1058,943,877,868,761,687,649 \mathrm{~cm}^{-1}$; HRMS (APCI) $m / z$ calculated for $\mathrm{C}_{26} \mathrm{H}_{17} \mathrm{Cl}_{2} \mathrm{~N}_{2} \mathrm{~S}_{2}[\mathrm{M}+\mathrm{H}]^{+}: 491.0205$, found 491.0202 .

$(6 Z, 12 Z)-N^{6}, N^{12}$-Bis(2-chlorophenyl)-6H,12H-dibenzo[b,f][1,5]dithiocine-6,12-diimine (7i)<smiles>Clc1ccccc1N=C1Sc2ccccc2C(=Nc2ccccc2Cl)c2ccccc2S1</smiles>
Yield: $72 \%$ (177 mg). White solid; mp $251-252{ }^{\circ} \mathrm{C} ;{ }^{1} \mathrm{H}$ NMR $(400 \mathrm{MHz}$, $\left.\mathrm{CDCl}_{3}\right) \delta 7.35(\mathrm{dd}, J=8.0,1.6 \mathrm{~Hz}, 2 \mathrm{H}), 7.48(\mathrm{dd}, J=7.6,1.8 \mathrm{~Hz}, 2 \mathrm{H}), 7.40-$ $7.36(\mathrm{~m}, 2 \mathrm{H}), 7.33-7.29(\mathrm{~m}, 2 \mathrm{H}), 7.27(\mathrm{dd}, J=8.0,1.2 \mathrm{~Hz}, 2 \mathrm{H}), 7.20-7.14$ (m, 6H); ${ }^{13} \mathrm{CNMR}\left(101 \mathrm{MHz}, \mathrm{CDCl}_{3}\right) \delta 169.0,146.5,142.8,136.2,130.8,130.2,130.2,129.1,128.0$, 127.6, 125.9, 124.3, 121.3; IR (KBr) v 3054, 1617, 1581, 1469, 1437, 1278, 1258, 1236, 1193, 1124, $1057,1030,951,832,759,726,684 \mathrm{~cm}^{-1}$; HRMS (APCI) $\mathrm{m} / z$ calculated for $\mathrm{C}_{26} \mathrm{H}_{17} \mathrm{Cl}_{2} \mathrm{~N}_{2} \mathrm{~S}_{2}[\mathrm{M}+\mathrm{H}]^{+}$: 491.0205, found 491.0206 .

$(6 Z, 12 Z)-2,8-D i m e t h y l-N^{6}, N^{12}$-diphenyl-6H,12H-dibenzo[b,f][1,5]dithiocine-6,12-diimine (7j)

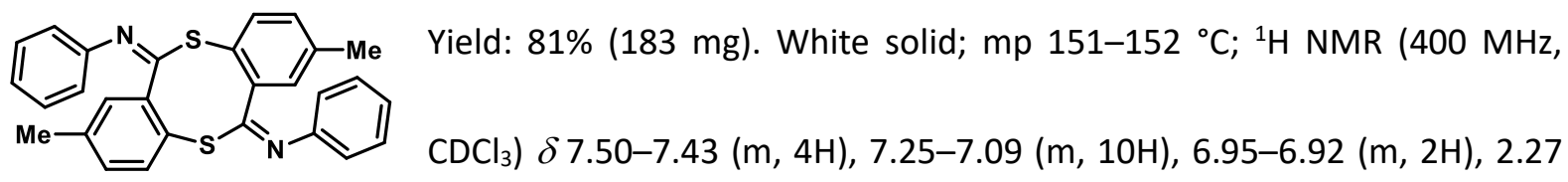
(s, 6H); ${ }^{13} \mathrm{C}$ NMR $\left(101 \mathrm{MHz}, \mathrm{CDCl}_{3}\right) \delta 167.5,149.7,148.1,143.1,141.0,135.9,130.6,129.3,124.9$, 121.7, 120.2, 21.2; IR (KBr) v 3055, 2987, 2921, 1618, 1593, 1585, 1481, 1434, 1385, 1307, 1160, $1103,1068,1025,998,978,853,815,714,691,638 \mathrm{~cm}^{-1}$; HRMS (APCI) $\mathrm{m} / z$ calculated for $\mathrm{C}_{28} \mathrm{H}_{23} \mathrm{~N}_{2} \mathrm{~S}_{2}$ $[\mathrm{M}+\mathrm{H}]^{+}:$451.1297, found 451.1325. 
(6Z,12Z)-2,8-Dimethyl- $N^{6}, N^{12}$-di-p-tolyl-6H,12H-dibenzo[b,f][1,5]dithiocine-6,12-diimine (7k)

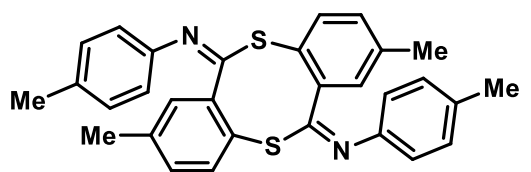

Yield: 79\% (189 mg). White solid; mp $145-146{ }^{\circ} \mathrm{C} ;{ }^{1} \mathrm{H}$ NMR (400

$\left.\mathrm{MHz}, \mathrm{CDCl}_{3}\right) \delta 7.29(\mathrm{~d}, J=8.0 \mathrm{~Hz}, 4 \mathrm{H}), 7.18(\mathrm{~s}, 2 \mathrm{H}), 7.09(\mathrm{~d}, J=8.4$

$\mathrm{Hz}, 6 \mathrm{H}), 6.92(\mathrm{~d}, J=7.6 \mathrm{~Hz}, 2 \mathrm{H}), 2.41(\mathrm{~s}, 6 \mathrm{H}), 2.26(\mathrm{~s}, 6 \mathrm{H}) ;{ }^{13} \mathrm{C} \mathrm{NMR}\left(101 \mathrm{MHz}, \mathrm{CDCl}_{3}\right) \delta 167.1,147.1$, $143.2,140.9,135.9,134.5,130.5,129.8,129.2,125.0,120.2,21.2,21.1$; IR (KBr) v 3080, 3048, 3071, $2920,1624,1503,1482,1468,1285,1239,1238,1203,1162,1104,1058,978,860,825,814 \mathrm{~cm}^{-1}$; HRMS (APCI) $m / z$ calculated for $\mathrm{C}_{30} \mathrm{H}_{27} \mathrm{~N}_{2} \mathrm{~S}_{2}[\mathrm{M}+\mathrm{H}]^{+}: 479.1610$, found 479.1585 .

$(6 Z, 12 Z)-N^{6}, N^{12}$-Bis(4-chlorophenyl)-2,8-dimethyl-6H,12H-dibenzo[b,f][1,5] dithiocine-6,12-diimine (기)

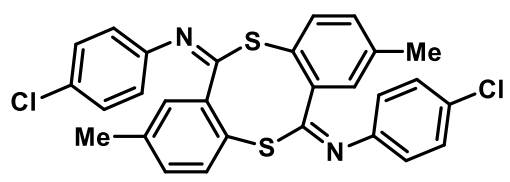
Yield: 73\% (189 mg). White solid; mp $198-199{ }^{\circ} \mathrm{C} ;{ }^{1} \mathrm{H}$ NMR (400 $\left.\mathrm{MHz}, \mathrm{CDCl}_{3}\right) \delta 7.44(\mathrm{~d}, J=8.0 \mathrm{~Hz}, 4 \mathrm{H}), 7.17(\mathrm{~s}, 2 \mathrm{H}), 7.10(\mathrm{~d}, J=7.2$ $\mathrm{Hz}, 6 \mathrm{H}), 6.95(\mathrm{~d}, J=7.2 \mathrm{~Hz}, 2 \mathrm{H}), 2.27(\mathrm{~s}, 6 \mathrm{H}) ;{ }^{13} \mathrm{CNMR}\left(101 \mathrm{MHz}, \mathrm{CDCl}_{3}\right) \delta 168.2,148.0,142.9,141.3$, $136.0,130.8,130.3,129.4,129.2,124.5,121.6,21.2$; IR (KBr) v 3088, 3051, 2914, 1610, 1586, 1482, $1465,1398,1284,1243,1202,1157,1085,1058,1009,976,888,861,682 \mathrm{~cm}^{-1}$; HRMS (APCI) $\mathrm{m} / \mathrm{z}$ calculated for $\mathrm{C}_{28} \mathrm{H}_{21} \mathrm{Cl}_{2} \mathrm{~N}_{2} \mathrm{~S}_{2}[\mathrm{M}+\mathrm{H}]^{+}:$519.0518, found 519.0528 .

(6Z,12Z)-2,8-Dimethoxy- $N^{6}, N^{12}$-diphenyl-6H,12H-dibenzo[b,f][1,5]dithiocine-6,12-diimine (7m)<smiles>COc1ccc2c(c1)C(=Nc1ccccc1)Sc1ccc(OC)cc1C(=Nc1ccccc1)N=C2</smiles>
6.67-6.64 (m, 2H), $3.77(\mathrm{~s}, 6 \mathrm{H}) ;{ }^{13} \mathrm{C} \mathrm{NMR}\left(101 \mathrm{MHz}, \mathrm{CDCl}_{3}\right) \delta 167.7,161.2,149.5,144.6,137.5,129.3$, $124.9,120.2,118.8,115.5,114.0,55.6$; IR (KBr) v 3013, 2968, 2937, 2834, 1611, 1590, 1484, 1472, 
$1401,1312,1272,1235,1219,1163,1063,1026,989,863,842,768,694 \mathrm{~cm}^{-1}$; HRMS (APCI) $\mathrm{m} / \mathrm{z}$ calculated for $\mathrm{C}_{28} \mathrm{H}_{23} \mathrm{~N}_{2} \mathrm{O}_{2} \mathrm{~S}_{2}[\mathrm{M}+\mathrm{H}]^{+}:$483.1195, found 483.1190 .

$(6 Z, 12 Z)-2,8-D i m e t h o x y-N^{6}, N^{12}$-di-p-tolyl-6H,12H-dibenzo[b,f][1,5]dithiocine-6,12-diimine (7n)

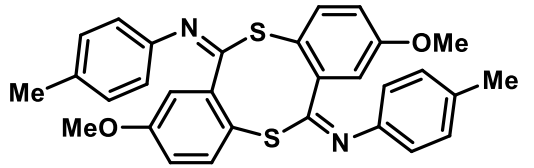

Yield: $79 \%$ (189 mg). White solid; mp $225-226{ }^{\circ} \mathrm{C} ;{ }^{1} \mathrm{H}$ NMR (400

$\left.\mathrm{MHz}, \mathrm{CDCl}_{3}\right) \delta 7.29(\mathrm{~d}, J=8.0 \mathrm{~Hz}, 4 \mathrm{H}), 7.13-7.08(\mathrm{~m}, 6 \mathrm{H}), 6.89(\mathrm{~d}$, $J=2.4 \mathrm{~Hz}, 2 \mathrm{H}), 6.64(\mathrm{dd}, J=8.4,2.8 \mathrm{~Hz}, 2 \mathrm{H}), 3.75(\mathrm{~s}, 6 \mathrm{H}), 2.41(\mathrm{~s}, 6 \mathrm{H}) ;{ }^{13} \mathrm{C} \mathrm{NMR}\left(101 \mathrm{MHz}, \mathrm{CDCl}_{3}\right) \delta$ $167.3,161.1,146.9,144.7,137.5,134.5,129.8,120.2,119.0,115.5,114.0,55.6,21.1$; IR (KBr) v 3026, 2959, 2934, 2837, 1625, 1592, 1565, 1504, 1467, 1417, 1189, 1176, 1298, 1211, 1080, 1025, 979, 855, 811, $663 \mathrm{~cm}^{-1}$; HRMS (APCl) $\mathrm{m} / z$ calculated for $\mathrm{C}_{30} \mathrm{H}_{27} \mathrm{~N}_{2} \mathrm{O}_{2} \mathrm{~S}_{2}[\mathrm{M}+\mathrm{H}]^{+}: 511.1508$, found 511.1530.

$(6 Z, 12 Z)-N^{6}, N^{12}$-Bis(4-chlorophenyl)-2,8-dimethoxy-6H,12H-dibenzo[b,f][1,5]dithiocine-6,12-diimin e (7o)

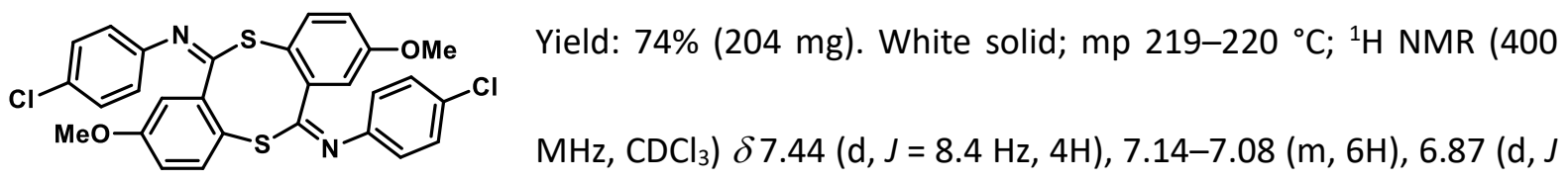
$=2.4 \mathrm{~Hz}, 2 \mathrm{H}), 6.67(\mathrm{dd}, J=8.8,2.8 \mathrm{~Hz}, 2 \mathrm{H}), 3.77(\mathrm{~s}, 6 \mathrm{H}) ;{ }^{13} \mathrm{C} \mathrm{NMR}\left(101 \mathrm{MHz}, \mathrm{CDCl}_{3}\right) \delta 168.4,161.3$, $147.9,144.4,137.6,130.3,129.5,121.6,118.4,115.7,114.1,55.6$; IR (KBr) v 3050, 2957, 2934, 2837, $1622,1589,1563,1484,1467,1415,1298,1279,1211,1080,1025,1012,979,853,681 \mathrm{~cm}^{-1}$; HRMS (APCI) $\mathrm{m} / \mathrm{z}$ calculated for $\mathrm{C}_{28} \mathrm{H}_{21} \mathrm{Cl}_{2} \mathrm{~N}_{2} \mathrm{O}_{2} \mathrm{~S}_{2}[\mathrm{M}+\mathrm{H}]^{+}: 551.0416$, found 551.0415 . 
$(6 Z, 12 Z)-2,8$-Dichloro- $N^{6}, N^{12}$-diphenyl-6H,12H-dibenzo[b,f][1,5]dithiocine-6,12-diimine $(7 p)$

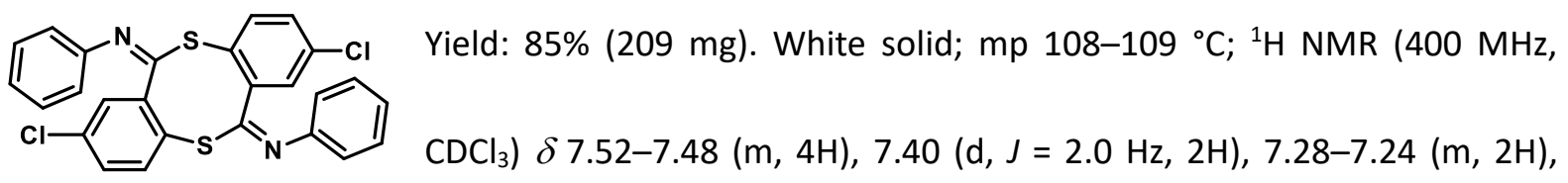

7.22-7.15 (m, 8H); ${ }^{13} \mathrm{C} \mathrm{NMR}\left(101 \mathrm{MHz}, \mathrm{CDCl}_{3}\right) \delta 164.4,149.0,144.3,137.3,137.2,130.2,129.4,128.9$, 126.6, 125.4, 120.0; IR (KBr) v 3047, 3018, 1615, 1591, 1553, 1484, 1456, 1384, 1231, 1186, 1097, 978, 919, 838, 773, 729, $694 \mathrm{~cm}^{-1}$; HRMS (APCl) $\mathrm{m} / z$ calculated for $\mathrm{C}_{26} \mathrm{H}_{17} \mathrm{Cl}_{2} \mathrm{~N}_{2} \mathrm{~S}_{2}[\mathrm{M}+\mathrm{H}]^{+}: 491.0205$, found 491.0206 .

$(6 Z, 12 Z)-2,8$-Dichloro- $N^{6}, N^{12}$-di-p-tolyl-6H,12H-dibenzo[b,f][1,5]dithiocine-6,12-diimine (7q)<smiles>Cc1ccc(/N=C2\Sc3ccc(Cl)cc3/C(=N\c3ccc(C)cc3)Sc3ccc(Cl)cc32)cc1</smiles>

Yield: $81 \%$ (210 mg). White solid; mp $198-199{ }^{\circ} \mathrm{C} ;{ }^{1} \mathrm{H}$ NMR (400 $\left.\mathrm{MHz}, \mathrm{CDCl}_{3}\right) \delta 7.39(\mathrm{~d}, J=2.0 \mathrm{~Hz}, 2 \mathrm{H}), 7.30(\mathrm{~d}, J=8.0 \mathrm{~Hz}, 4 \mathrm{H})$, 7.21-7.14 (m, 4H), 7.08-7.06 (m, 4H), $\left.2.41(\mathrm{~s}, 6 \mathrm{H}) ;{ }^{13} \mathrm{C} \mathrm{NMR} \mathrm{(101} \mathrm{MHz,} \mathrm{CDCl}_{3}\right) \delta 167.8,148.0,144.2$ $142.7,131.2,130.5,130.2,129.4,127.6,124.8,121.4,21.5 ; \mathrm{IR}(\mathrm{KBr})$ v 3056, 3021, 2916, 1627, 1577, $1551,1501,1456,1386,1252,1229,1186,1167,1094,1052,973,960,846,820 \mathrm{~cm}^{-1}$; HRMS (APCI) $m / z$ calculated for $\mathrm{C}_{28} \mathrm{H}_{21} \mathrm{Cl}_{2} \mathrm{~N}_{2} \mathrm{~S}_{2}[\mathrm{M}+\mathrm{H}]^{+}:$519.0518, found 519.0519 .

$(6 Z, 12 Z)-2,8-D i c h l o r o-N^{6}, N^{12}$-bis(4-chlorophenyl)-6H,12H-dibenzo[b,f][1,5]dithiocine-6,12-diimine (7r)

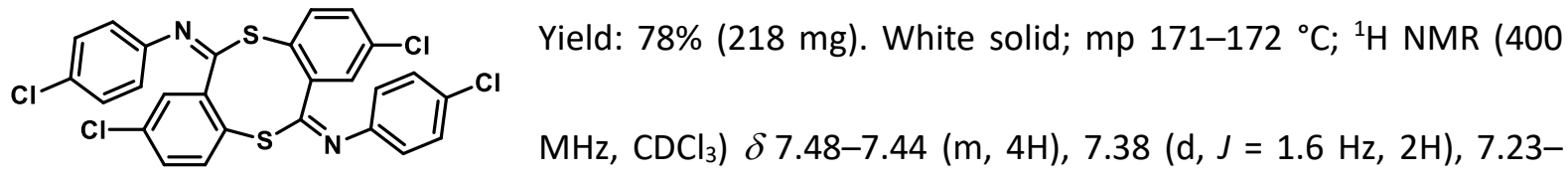
$7.18(\mathrm{~m}, 4 \mathrm{H}), 7.09-7.06(\mathrm{~m}, 4 \mathrm{H}) ;{ }^{13} \mathrm{C} \mathrm{NMR}\left(101 \mathrm{MHz}, \mathrm{CDCl}_{3}\right) \delta 165.1,147.3,144.1,137.4,137.3,130.9$, $130.5,129.6,128.9,126.1,121.4$; IR (KBr) v 3086, 3061, 3028, 1625, 1577, 1554, 1479, 1385, 1272, 
$1234,1185,1097,1010,976,886,842,823 \mathrm{~cm}^{-1}$; HRMS (APCI) $\mathrm{m} / z$ calculated for $\mathrm{C}_{26} \mathrm{H}_{15} \mathrm{Cl}_{4} \mathrm{~N}_{2} \mathrm{~S}_{2}[\mathrm{M}+$ $H]^{+}: 558.9425$, found 558.9424 .

$(6 Z, 12 Z)-4,10-$ Dimethyl- $N^{6}, N^{12}$-di-p-tolyl-6H,12H-dibenzo[b,f][1,5]dithiocine-6,12-diimine (7s)<smiles>Cc1ccc(/N=C2\Sc3c(cccc3[N+](=O)[O-])/C(=N\c3ccc(C)cc3)Sc3ccccc32)cc1</smiles>

Yield: $72 \%$ (172 mg). White solid; mp $210-211{ }^{\circ} \mathrm{C} ;{ }^{1} \mathrm{H}$ NMR (400

$\left.\mathrm{MHz}, \mathrm{CDCl}_{3}\right) \delta 7.30(\mathrm{~d}, J=8.0 \mathrm{~Hz}, 4 \mathrm{H}), 7.19-7.17(\mathrm{~m}, 2 \mathrm{H}), 7.14-$ $7.10(\mathrm{~m}, 6 \mathrm{H}), 7.01-6.99(\mathrm{~m}, 2 \mathrm{H}), 2.42(\mathrm{~s}, 6 \mathrm{H}), 2.25(\mathrm{~s}, 6 \mathrm{H}) ;{ }^{13} \mathrm{C}$ NMR $\left(101 \mathrm{MHz}, \mathrm{CDCl}_{3}\right) \delta 166.8,147.2,143.6,142.6,134.4,130.9,130.3,129.9,128.2,124.9,120.0$ 21.5, 21.1; IR (KBr) v 3047, 2982, 2919, 2865, 1629, 1501, 1457, 1270, 1211, 1195, 1165, 1106, 1017, $972,865,825,786,759,711 \mathrm{~cm}^{-1}$; HRMS (APCI) $\mathrm{m} / z$ calculated for $\mathrm{C}_{30} \mathrm{H}_{27} \mathrm{~N}_{2} \mathrm{~S}_{2}[\mathrm{M}+\mathrm{H}]^{+}:$479.1610, found 479.1585 .

$(6 Z, 12 Z)-N^{6}, N^{12}$-Bis(4-chlorophenyl)-4,10-dimethyl-6H,12H-dibenzo[b,f][1,5]dithiocine-6,12-diimine $(7 \mathrm{t})$<smiles>O=[N+]([O-])c1cccc2c1S/C(=N/c1ccc(Cl)cc1)c1ccccc1/S2=N/c1ccc(Cl)cc1</smiles>
Yield: $76 \%$ (197 mg). White solid; mp 208-209 ${ }^{\circ} \mathrm{C} ;{ }^{1} \mathrm{H}$ NMR (400 $\left.\mathrm{MHz}, \mathrm{CDCl}_{3}\right) \delta 7.47-7.43(\mathrm{~m}, 4 \mathrm{H}), 7.19-7.15(\mathrm{~m}, 4 \mathrm{H}), 7.13-7.09(\mathrm{~m}$, 4H), 7.05-7.02 (m, 2H), $\left.2.26(\mathrm{~s}, 6 \mathrm{H}) ;{ }^{13} \mathrm{C} \mathrm{NMR} \mathrm{(101} \mathrm{MHz,} \mathrm{CDCl}_{3}\right) \delta$ 164.0, 146.4, 144.4, 137.3, 137.1, 135.1, 130.1, 130.0, 128.9, 126.7, 120.1, 21.1; IR (KBr) v 3051, $2974,2949,2916,1611,1482,1458,1378,1270,1197,1089,1010,973,865,829,785,740,720$, $657 \mathrm{~cm}^{-1}$; HRMS (APCI) $\mathrm{m} / z$ calculated for $\mathrm{C}_{28} \mathrm{H}_{21} \mathrm{Cl}_{2} \mathrm{~N}_{2} \mathrm{~S}_{2}[\mathrm{M}+\mathrm{H}]^{+}: 519.0518$, found 519.0518 . 


\section{NMR spectra}

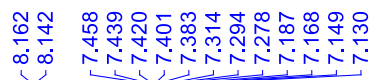

$\stackrel{\circ}{\circ}$

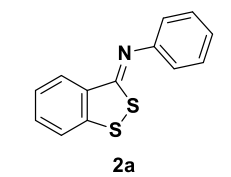

${ }^{1} \mathrm{H} \mathrm{NMR}\left(400 \mathrm{MHz}, \mathrm{CDCl}_{3}\right)$

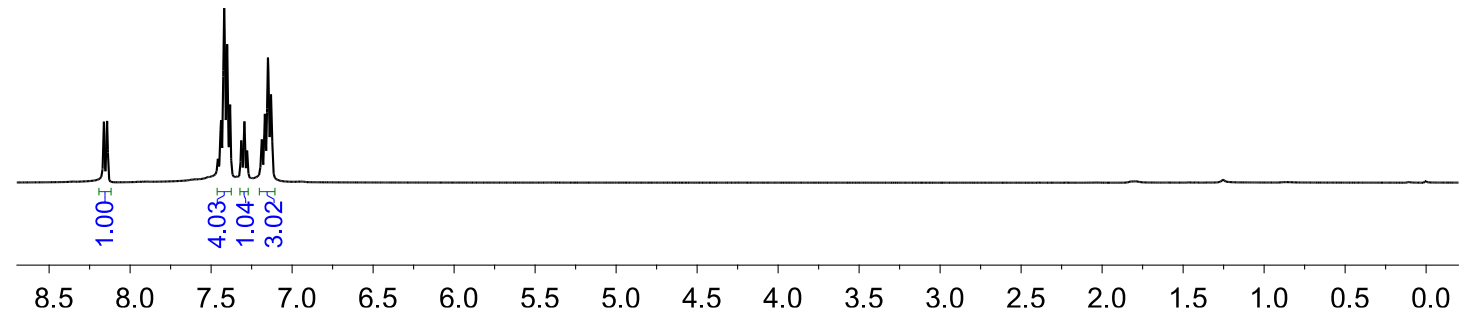

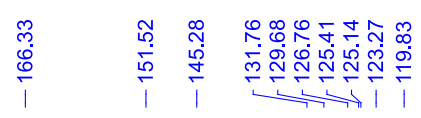

$\stackrel{\circ}{\stackrel{8}{0}}$

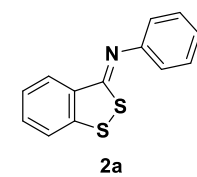

${ }^{13} \mathrm{C}$ NMR $\left(101 \mathrm{MHz}, \mathrm{CDCl}_{3}\right)$

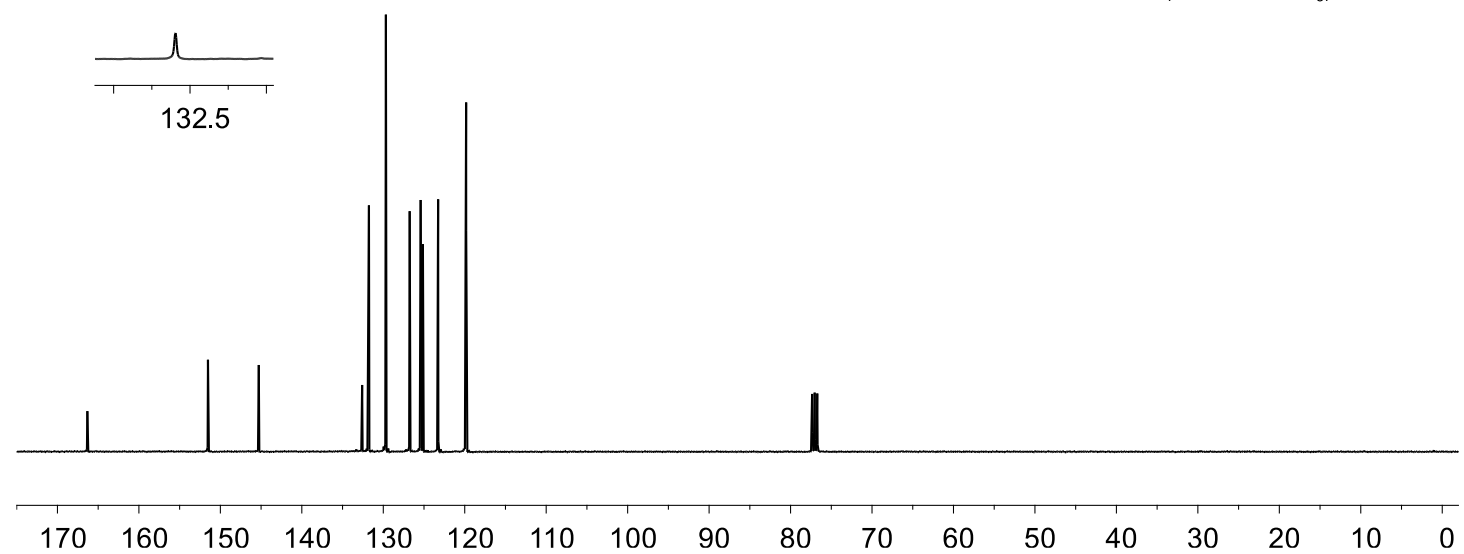




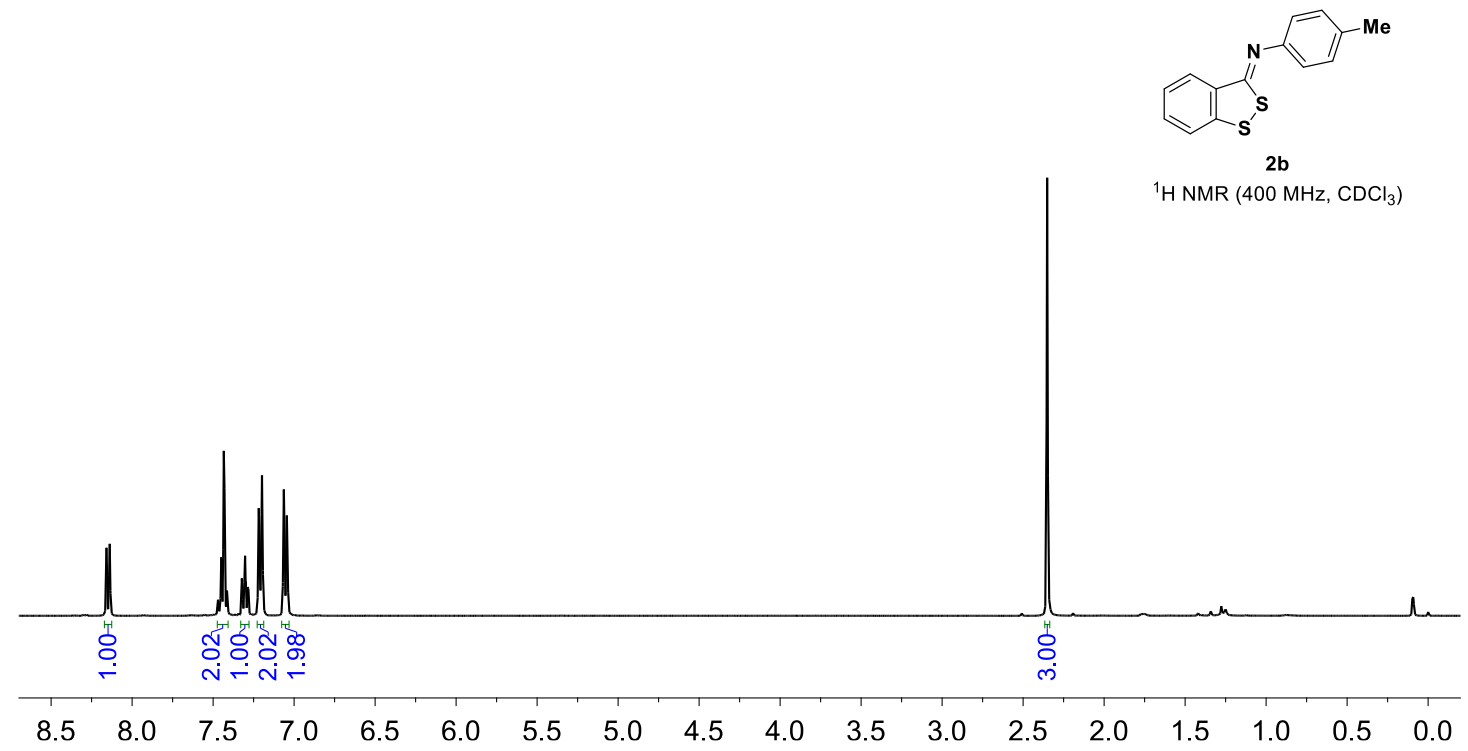

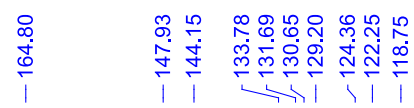

$\begin{array}{ll}0 & 8 \\ 0 & 0 \\ 0 & 0\end{array}$

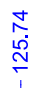
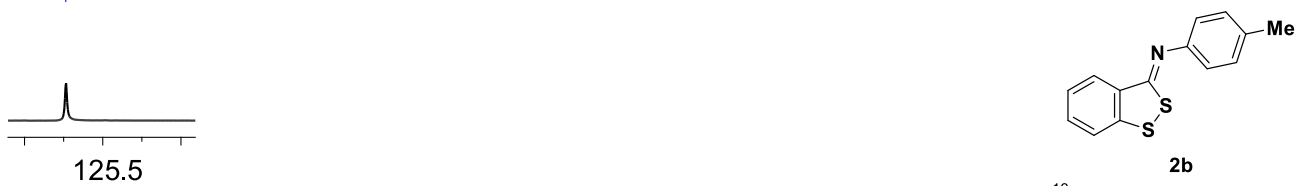

${ }^{13} \mathrm{C} \mathrm{NMR}\left(101 \mathrm{MHz}, \mathrm{CDCl}_{3}\right)$

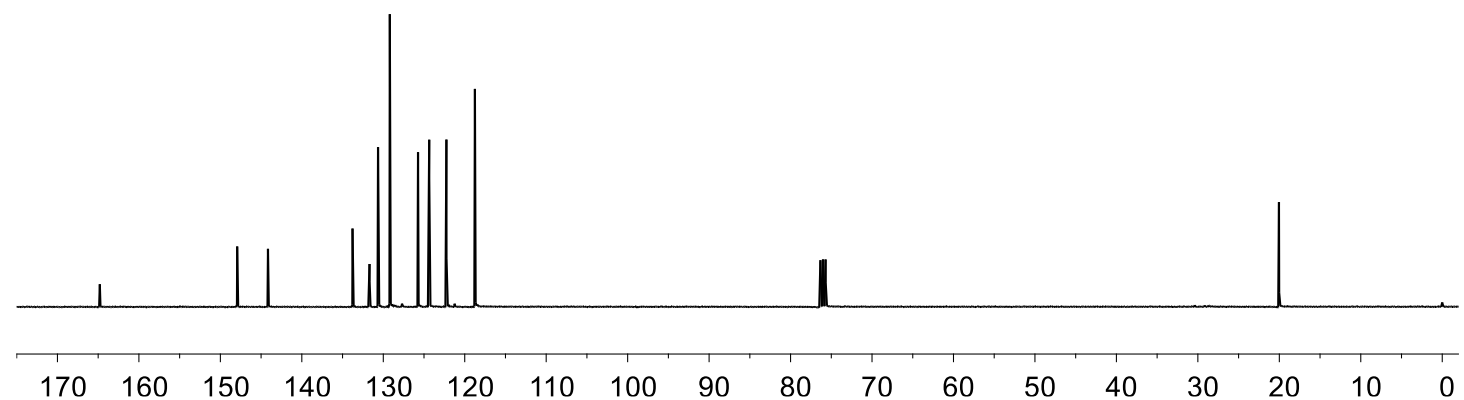




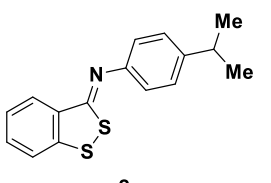

${ }^{1} \mathrm{H} \mathrm{NMR}\left(400 \mathrm{MHz}, \mathrm{CDCl}_{3}\right)$

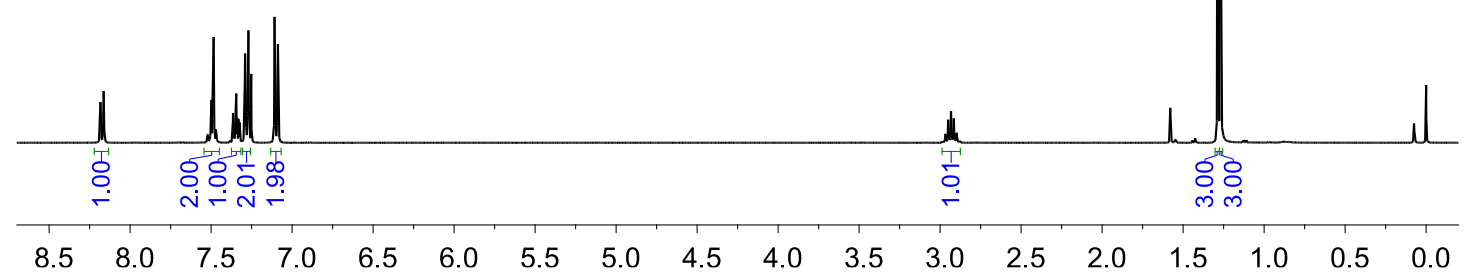

ll $\quad$ i
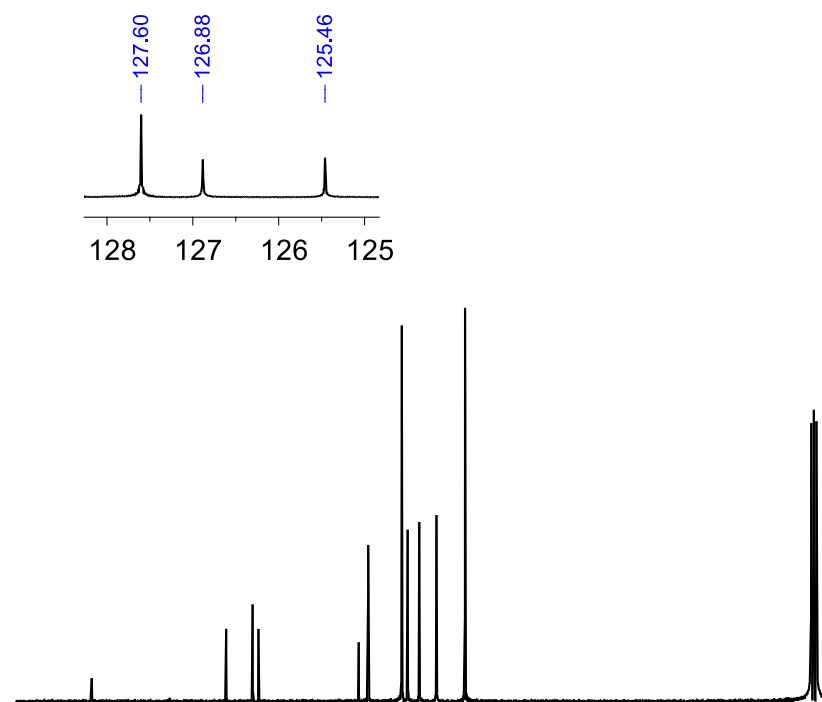

170 $\begin{array}{lll}\nwarrow & \stackrel{2}{0} & 8 \\ \stackrel{m}{j} & \stackrel{0}{+} & 0 \\ 1 & 1 & 1\end{array}$

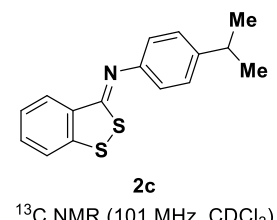

${ }^{13} \mathrm{C} \mathrm{NMR}\left(101 \mathrm{MHz}, \mathrm{CDCl}_{3}\right)$ 


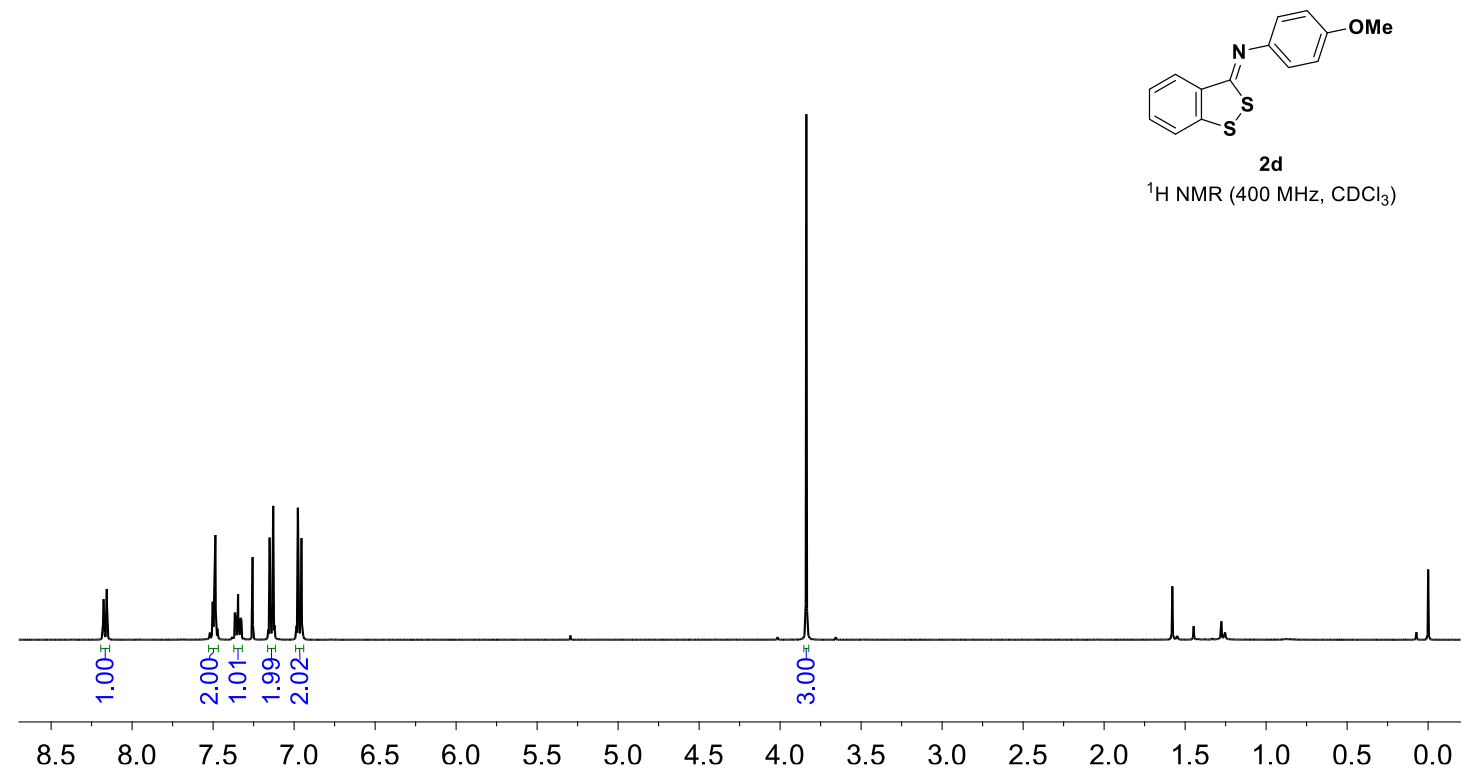

in

离
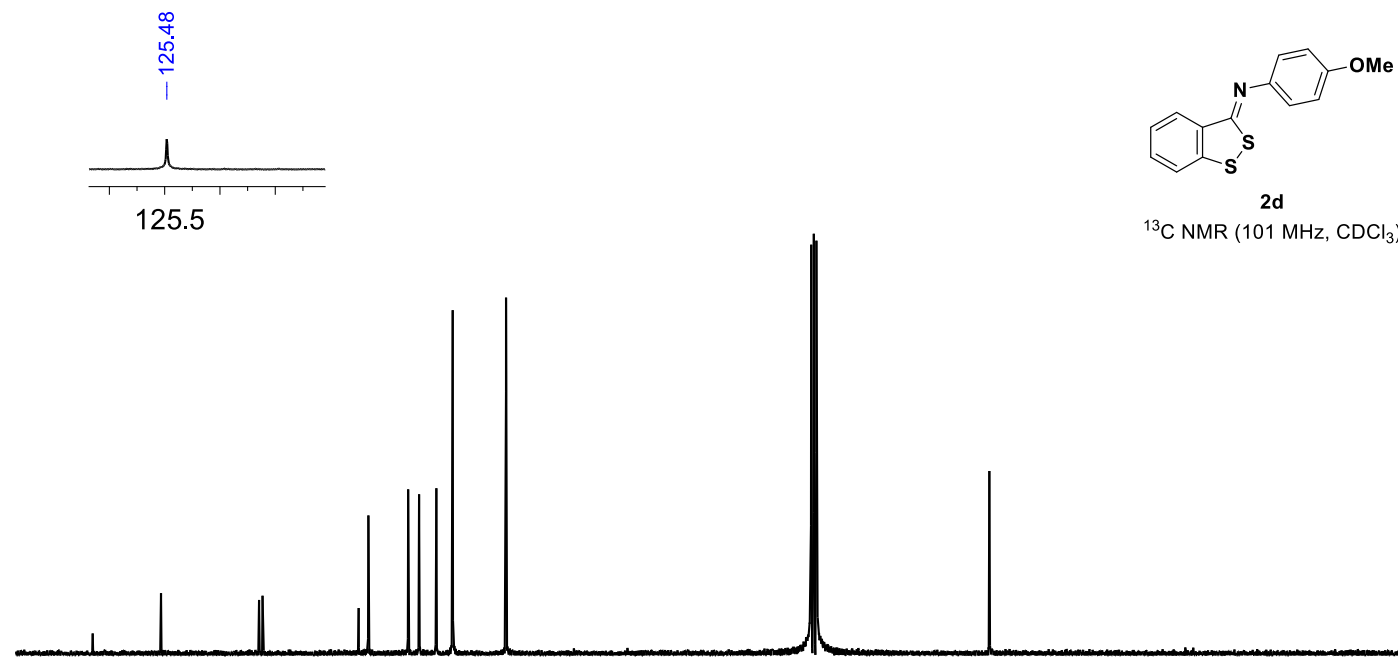

$\left.{ }^{13} \mathrm{C} \mathrm{NMR} \mathrm{(101} \mathrm{MHz,} \mathrm{CDCl}_{3}\right)$

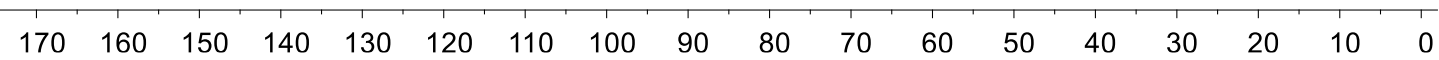



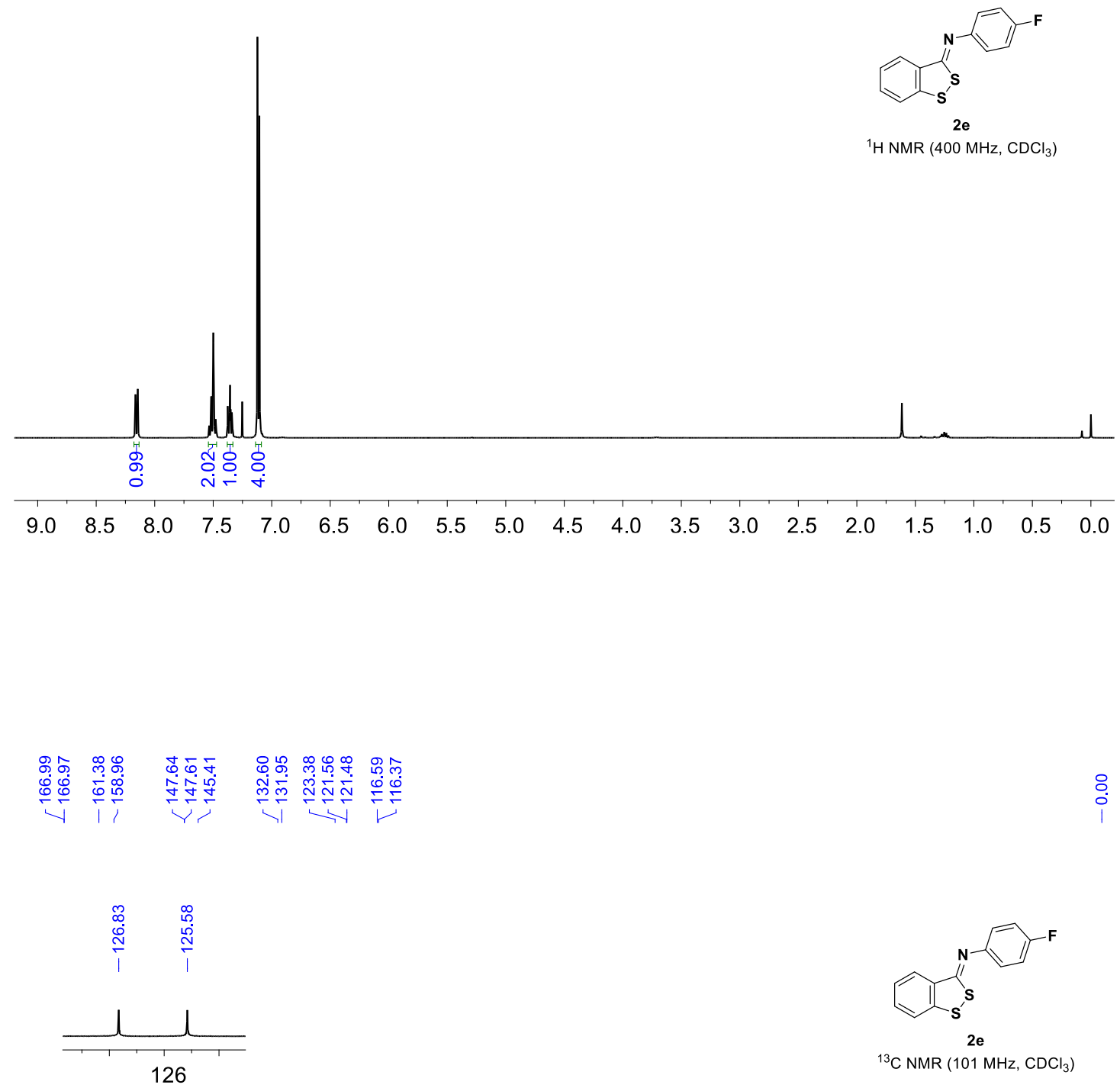

$\left.{ }^{13} \mathrm{C} \mathrm{NMR} \mathrm{(101} \mathrm{MHz,} \mathrm{CDCl}_{3}\right)$

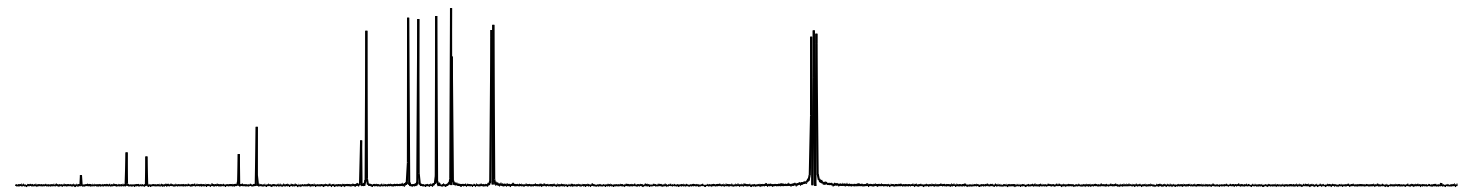

$\begin{array}{llllllllllllllllll}170 & 160 & 150 & 140 & 130 & 120 & 110 & 100 & 90 & 80 & 70 & 60 & 50 & 40 & 30 & 20 & 10 & 0\end{array}$ 


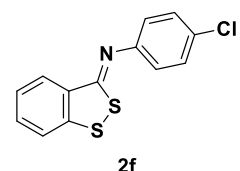

${ }^{1} \mathrm{H}$ NMR $\left(400 \mathrm{MHz}, \mathrm{CDCl}_{3}\right)$

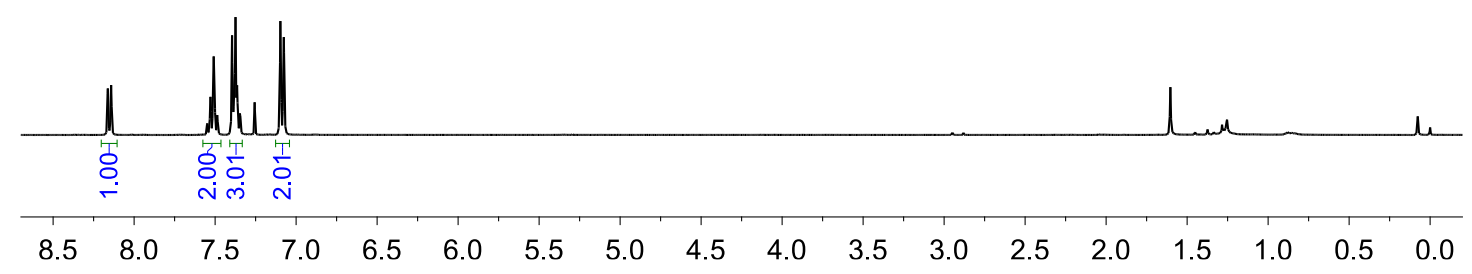

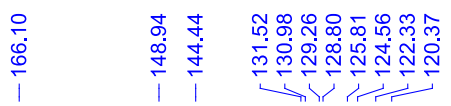

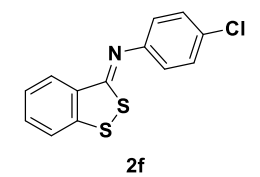

${ }^{13} \mathrm{C} \mathrm{NMR}\left(101 \mathrm{MHz}, \mathrm{CDCl}_{3}\right)$

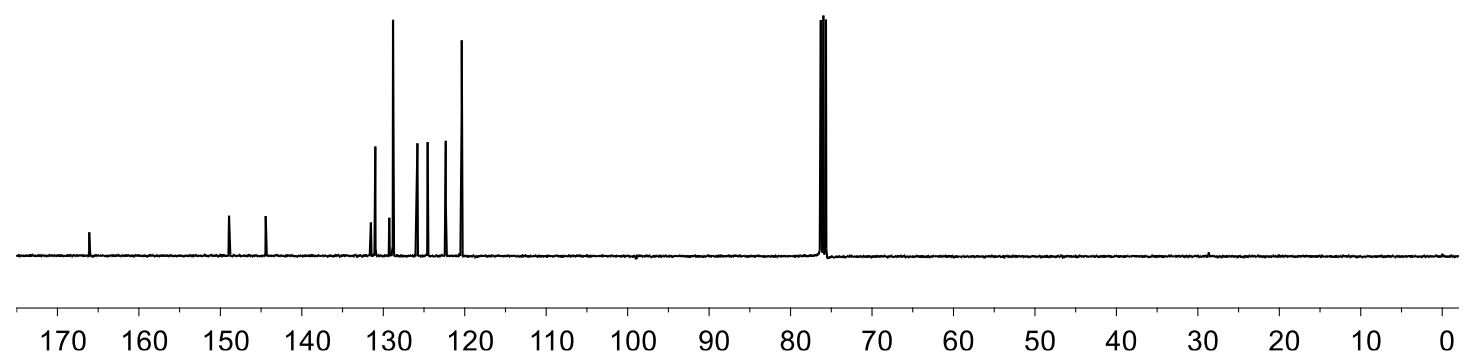




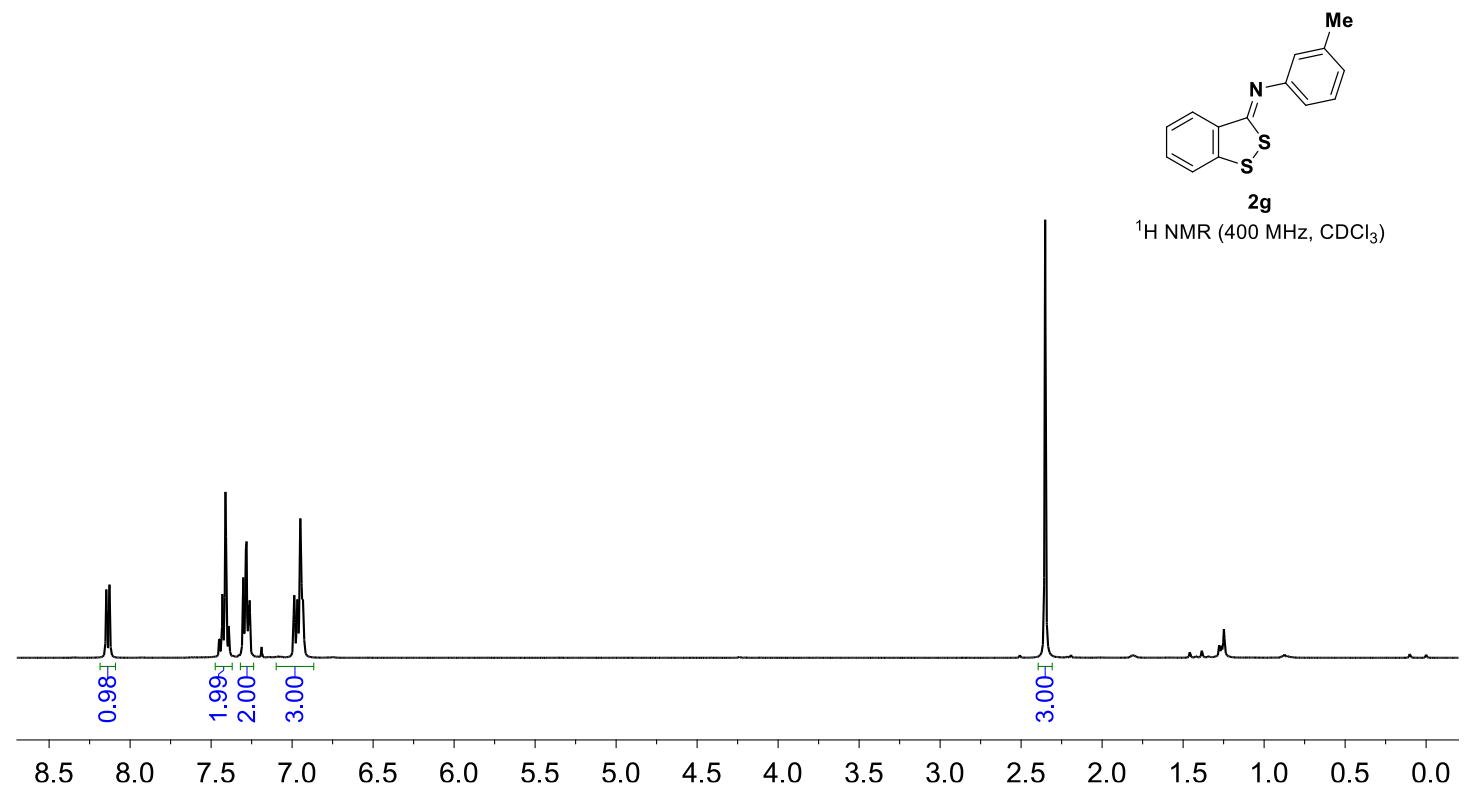

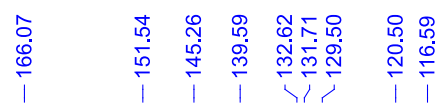
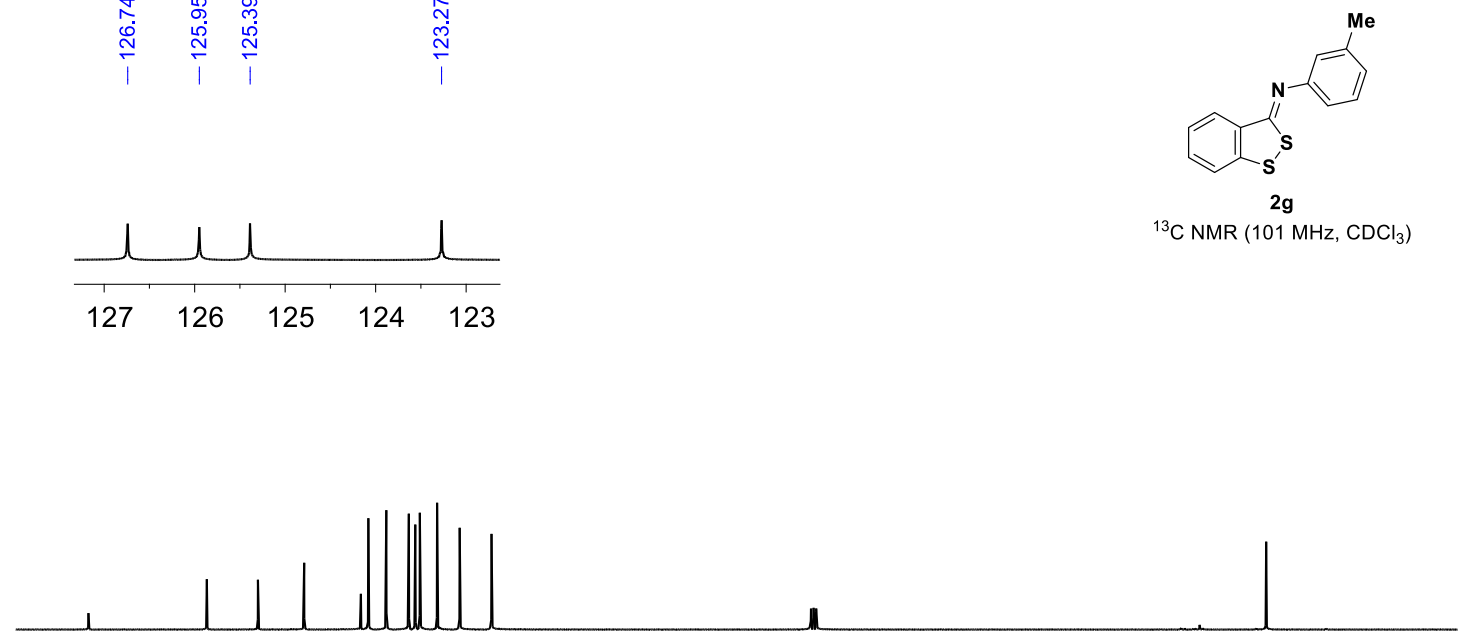

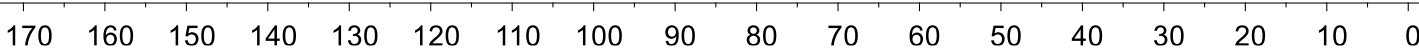




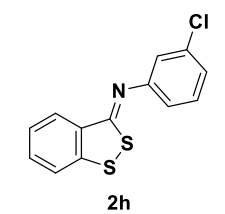

${ }^{1} \mathrm{H} \mathrm{NMR}\left(400 \mathrm{MHz}, \mathrm{CDCl}_{3}\right)$

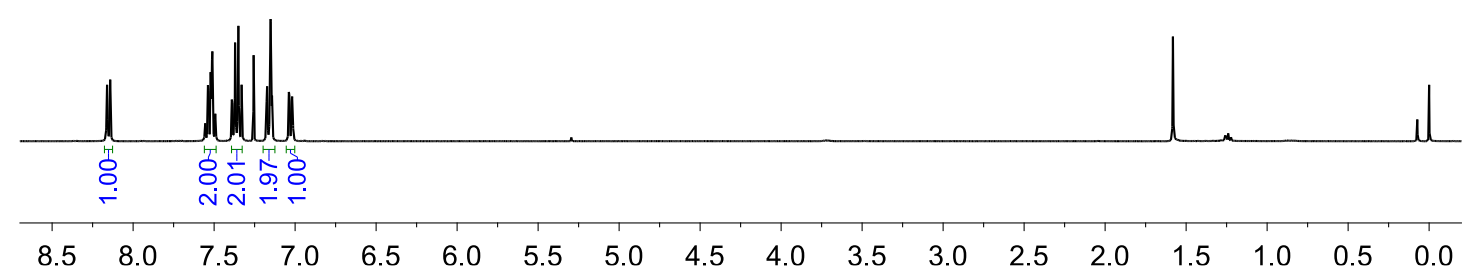

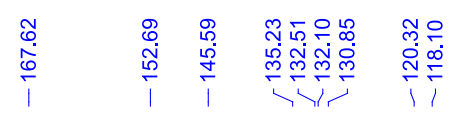
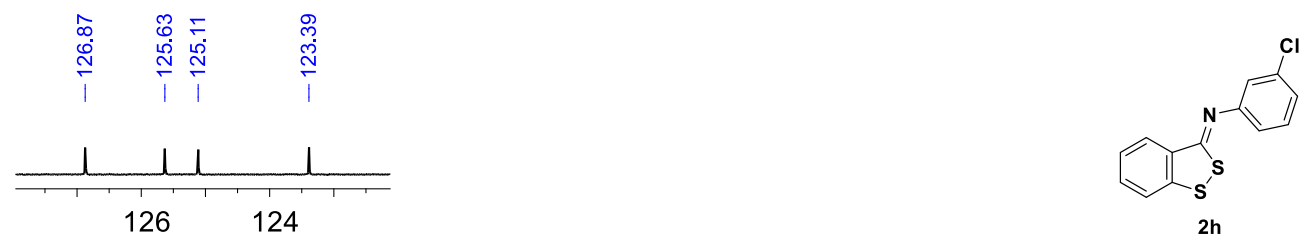

$\left.{ }^{13} \mathrm{C} \mathrm{NMR} \mathrm{(101} \mathrm{MHz,} \mathrm{CDCl}_{3}\right)$

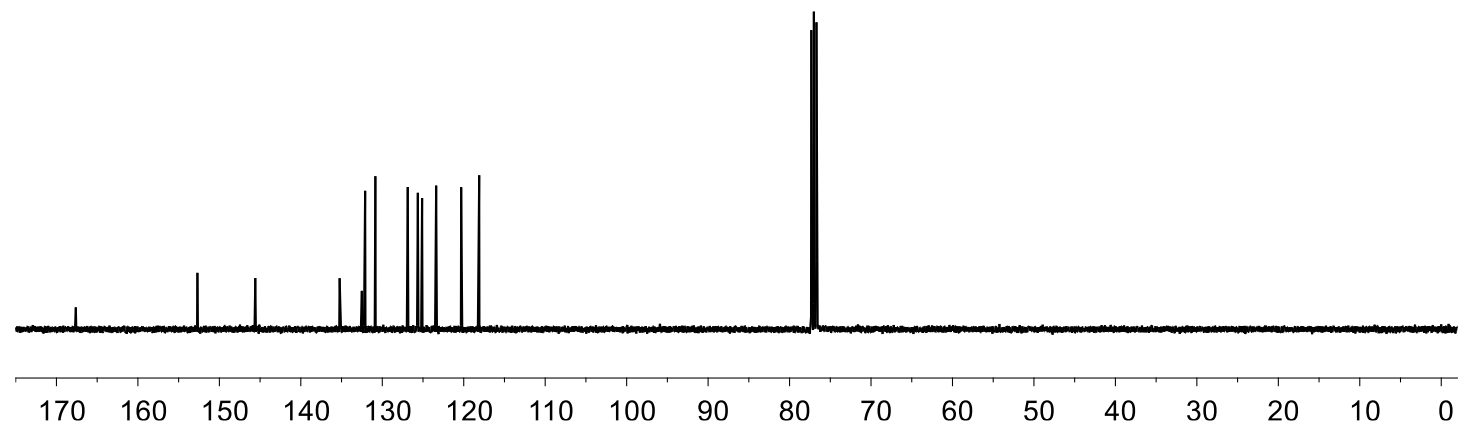




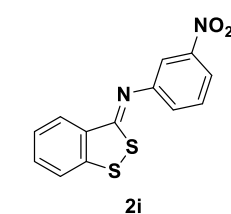

${ }^{1} \mathrm{H} \mathrm{NMR}\left(400 \mathrm{MHz}, \mathrm{CDCl}_{3}\right)$

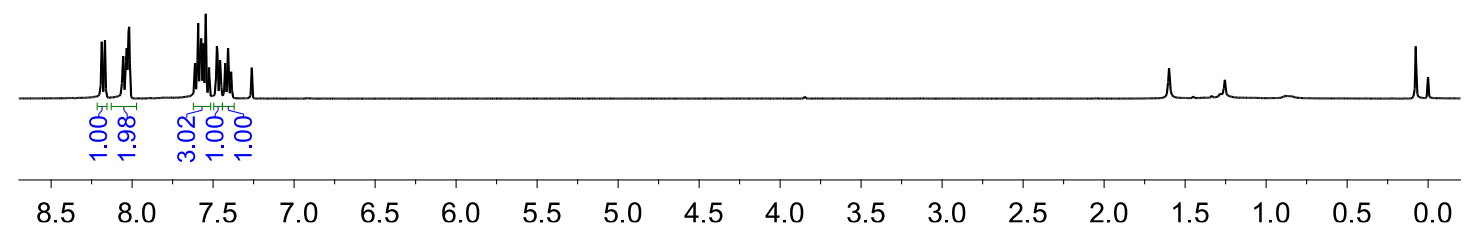

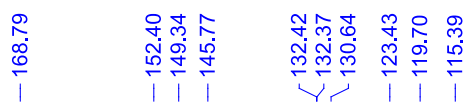
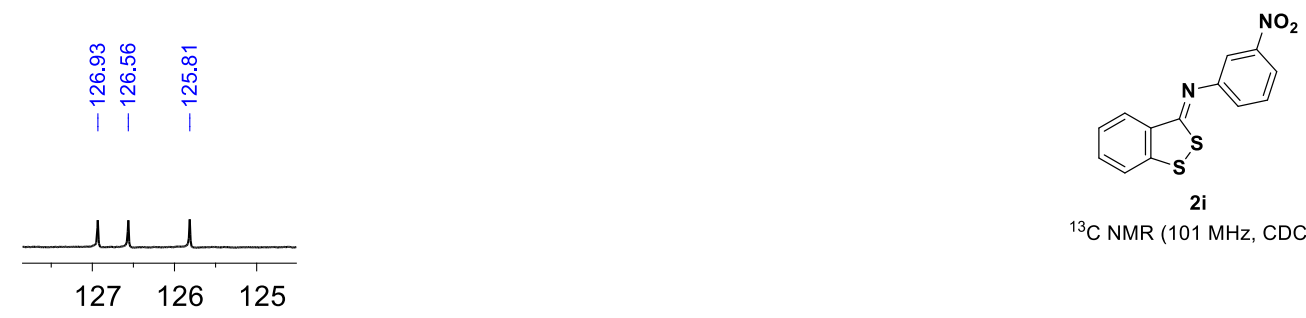

${ }^{13} \mathrm{C} \mathrm{NMR}\left(101 \mathrm{MHz}, \mathrm{CDCl}_{3}\right)$

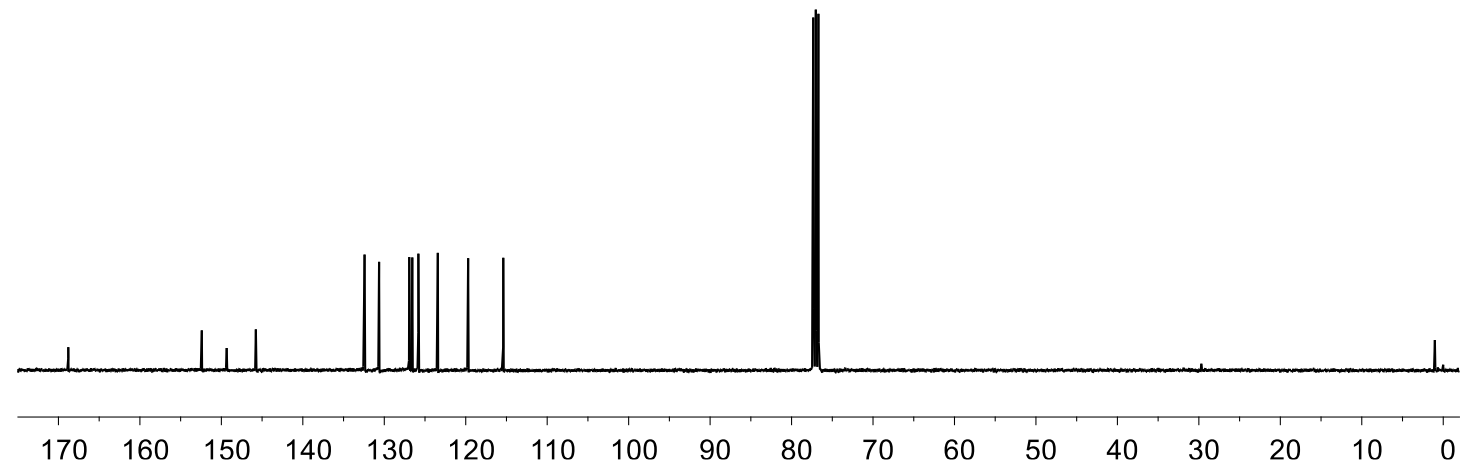




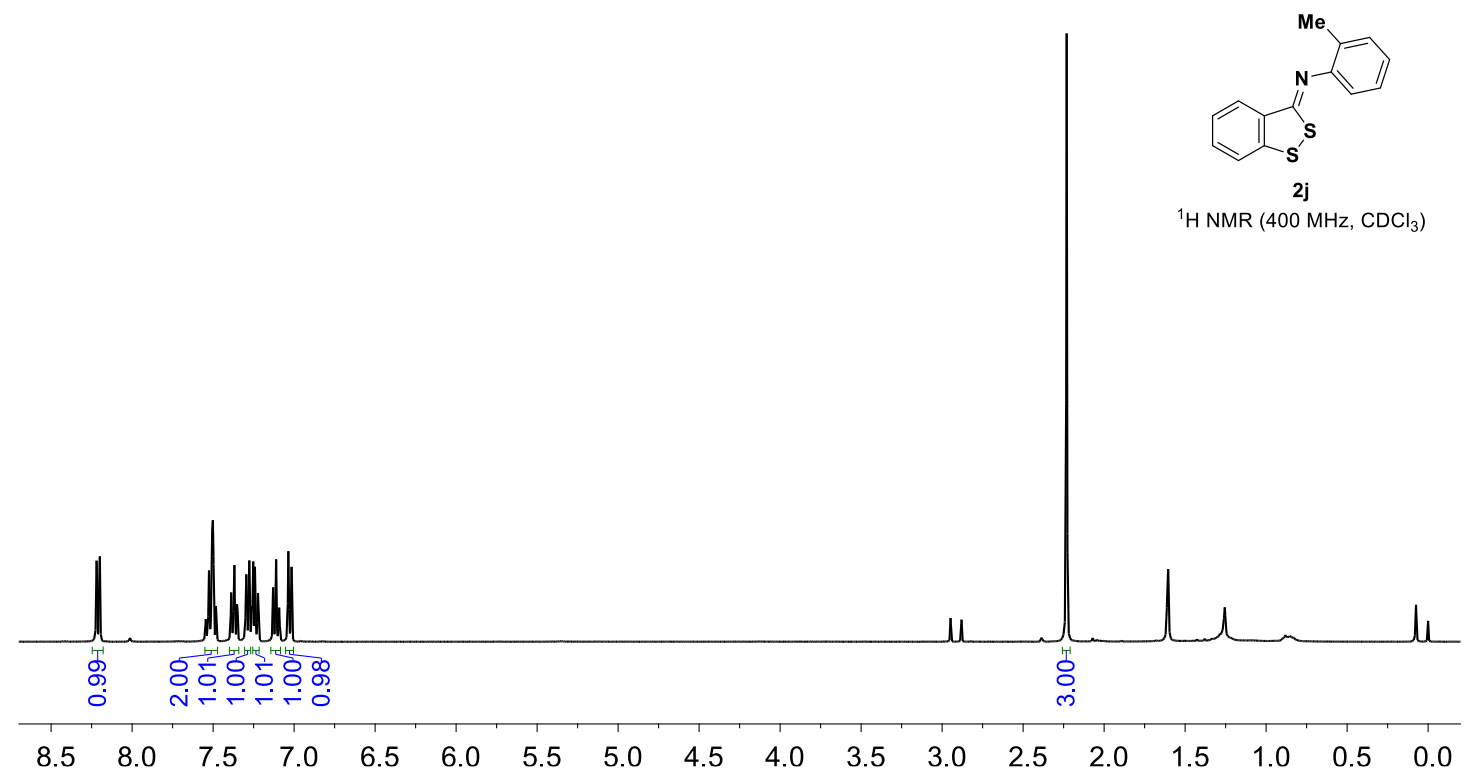

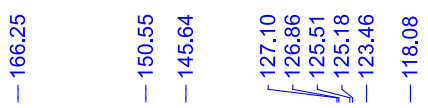

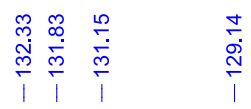
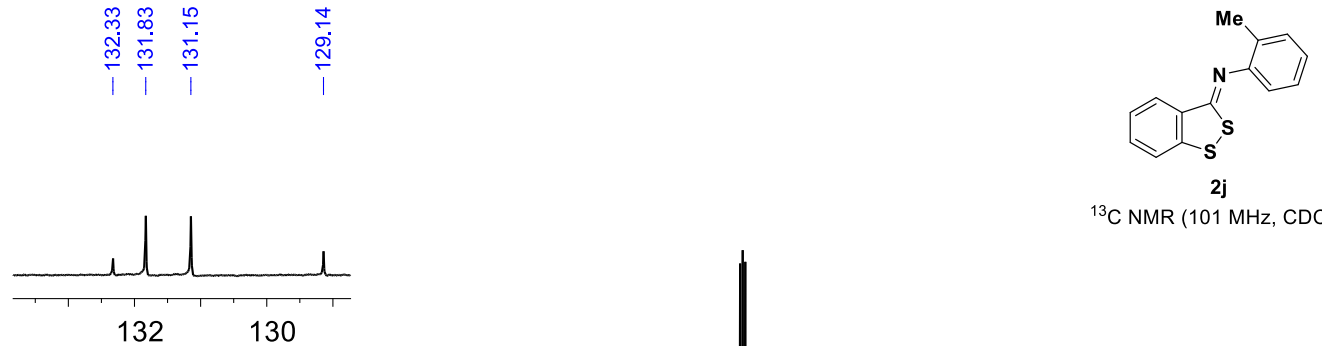

${ }^{13} \mathrm{C}$ NMR $\left(101 \mathrm{MHz}, \mathrm{CDCl}_{3}\right)$

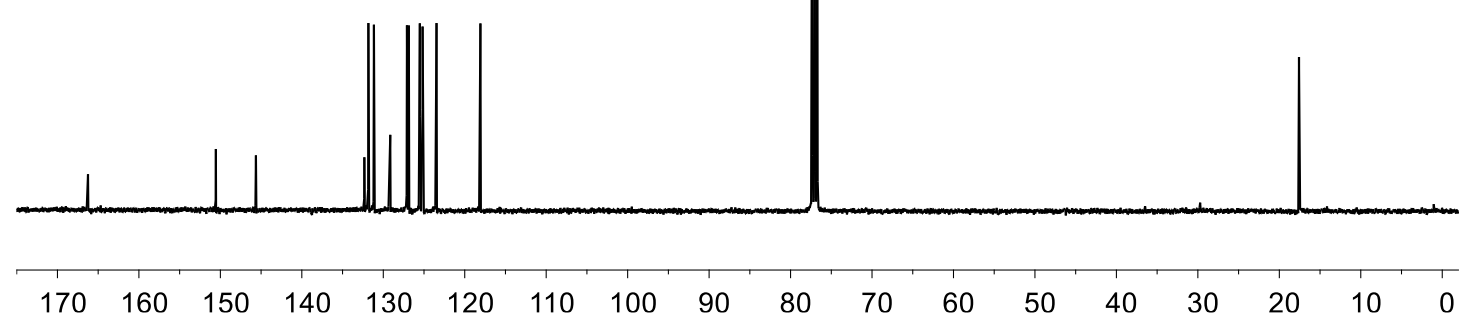




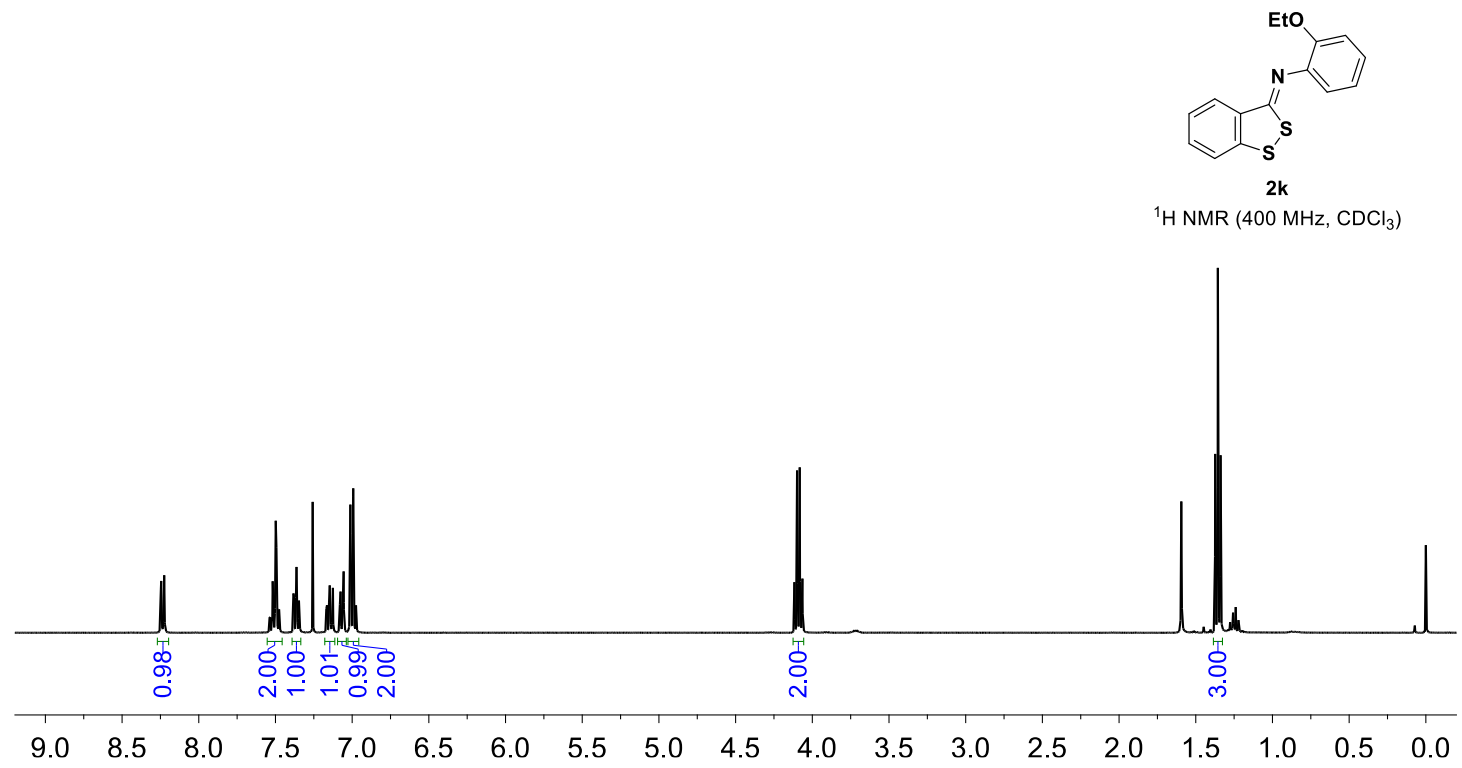

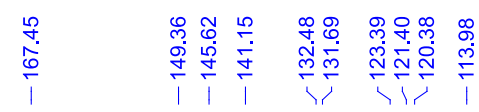

$\underset{\substack{\infty \\ 0 \\ 0}}{\substack{+i}}$

$\begin{array}{ll}\stackrel{\infty}{\infty} & 8 \\ \stackrel{+}{+} & 0 \\ 1 & i\end{array}$
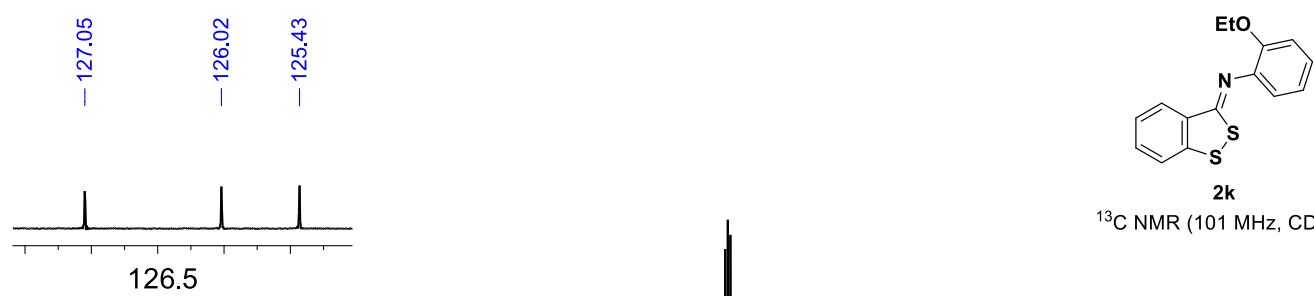

${ }^{13} \mathrm{C} \mathrm{NMR}\left(101 \mathrm{MHz}, \mathrm{CDCl}_{3}\right)$

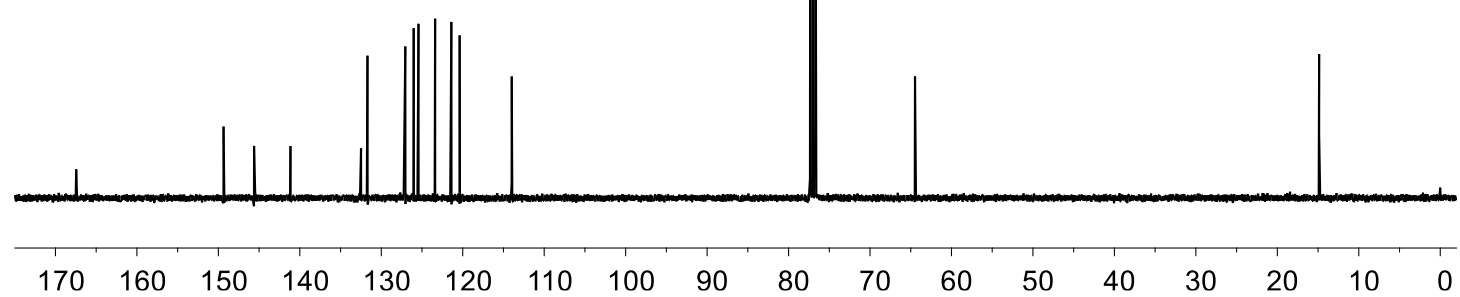




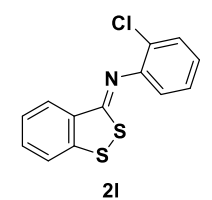

${ }^{1} \mathrm{H} \mathrm{NMR}\left(400 \mathrm{MHz}, \mathrm{CDCl}_{3}\right)$

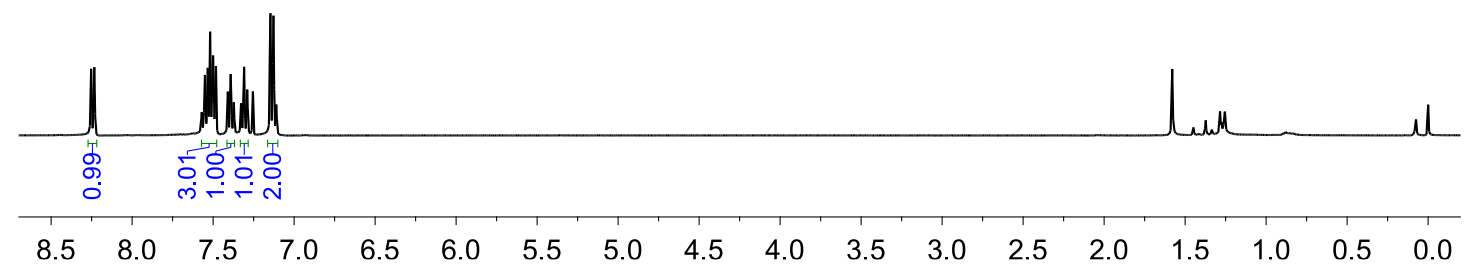

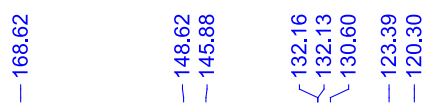
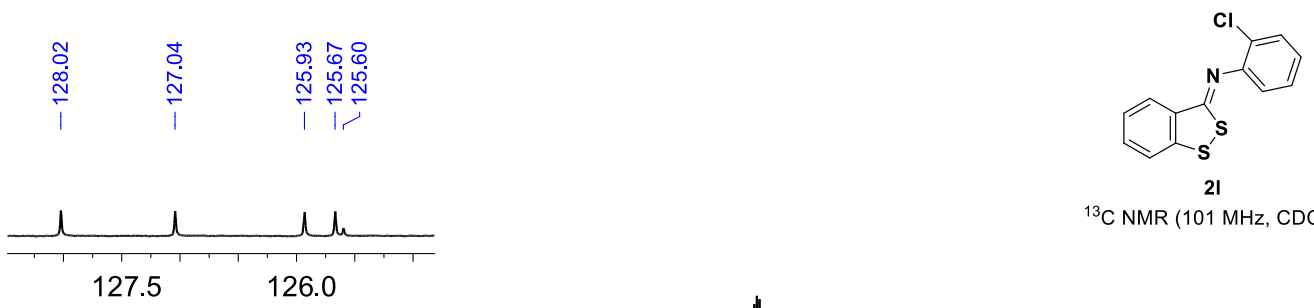

${ }^{13} \mathrm{C}$ NMR $\left(101 \mathrm{MHz}, \mathrm{CDCl}_{3}\right)$

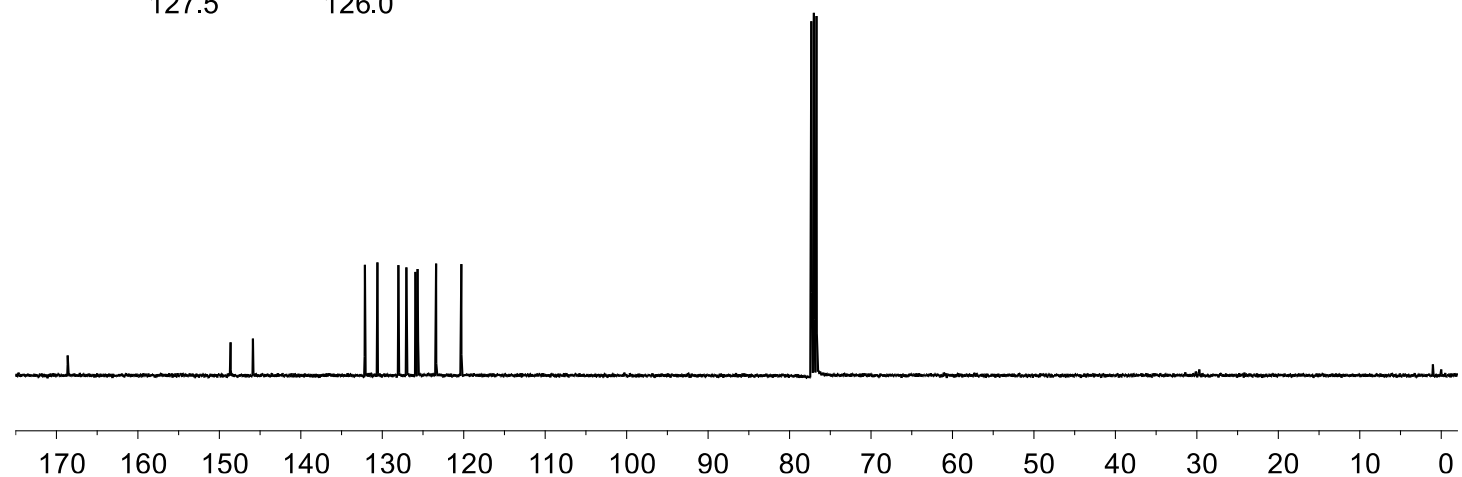




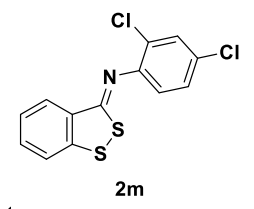

${ }^{1} \mathrm{H} \mathrm{NMR}\left(400 \mathrm{MHz}, \mathrm{CDCl}_{3}\right)$
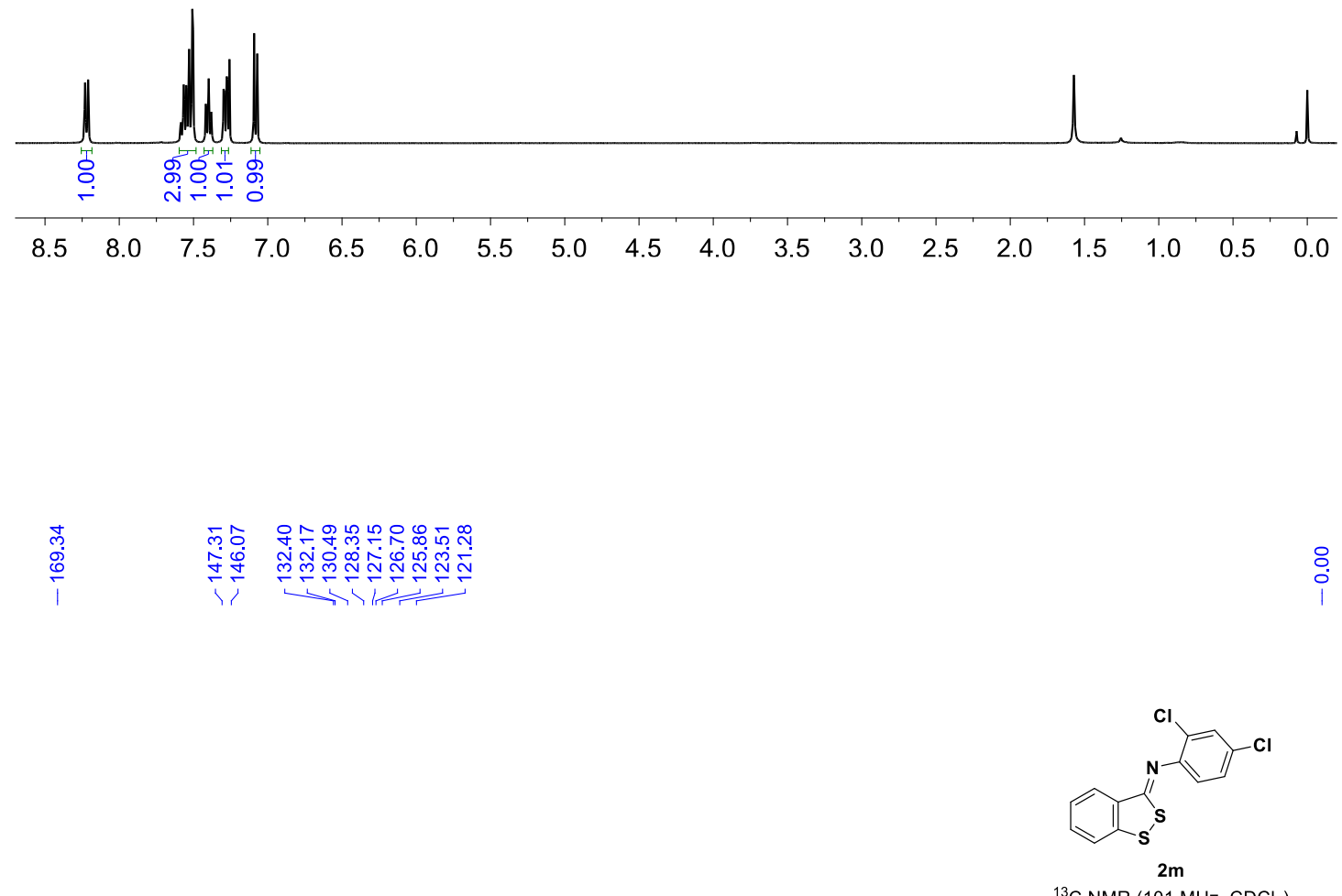

${ }^{13} \mathrm{C}$ NMR $\left(101 \mathrm{MHz}, \mathrm{CDCl}_{3}\right)$

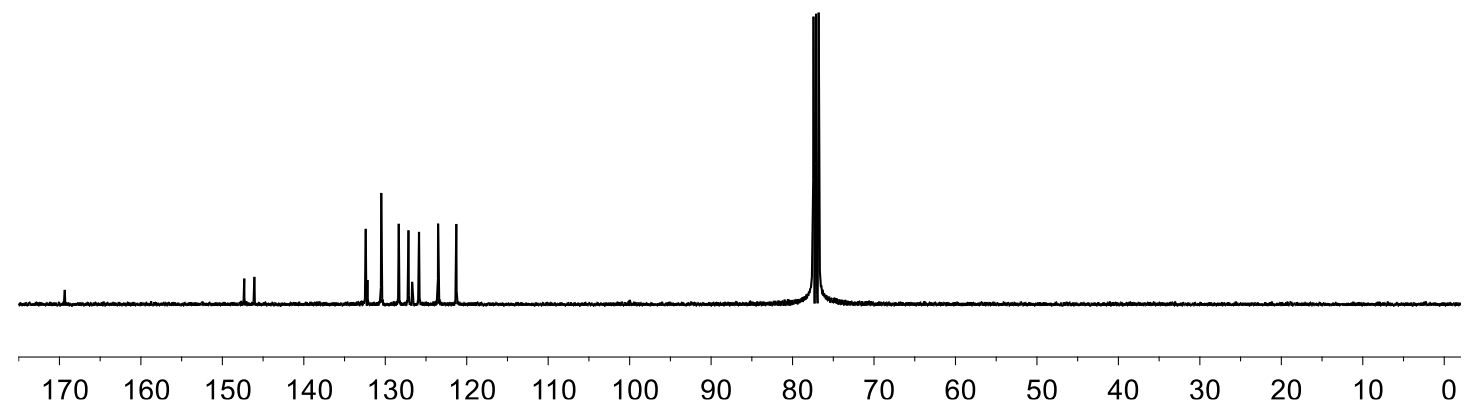




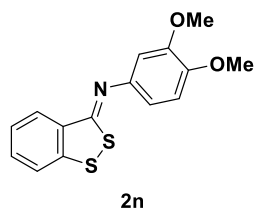

${ }^{1} \mathrm{H} \mathrm{NMR}\left(400 \mathrm{MHz}, \mathrm{CDCl}_{3}\right)$
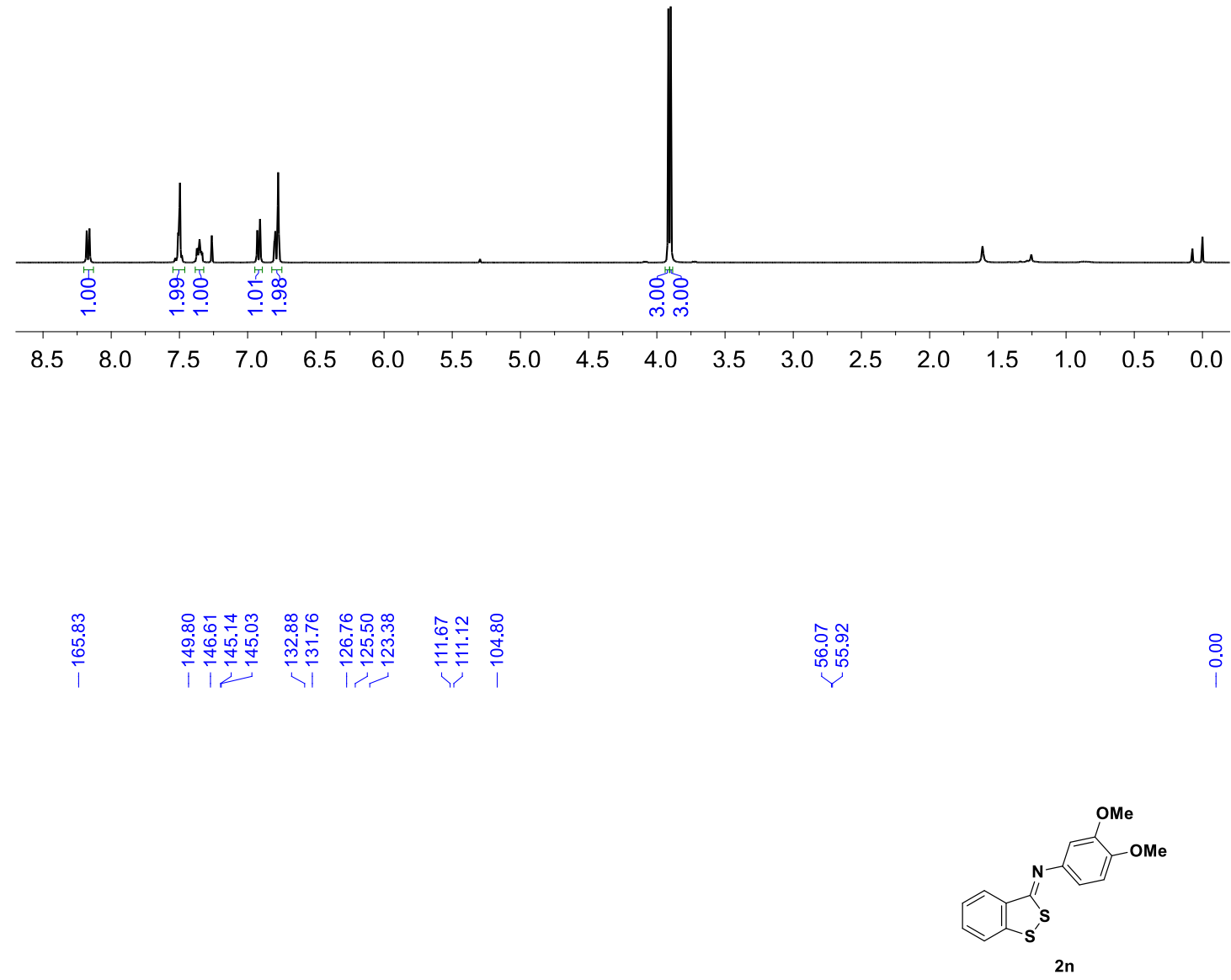

${ }^{13} \mathrm{C} \mathrm{NMR}\left(101 \mathrm{MHz}, \mathrm{CDCl}_{3}\right)$

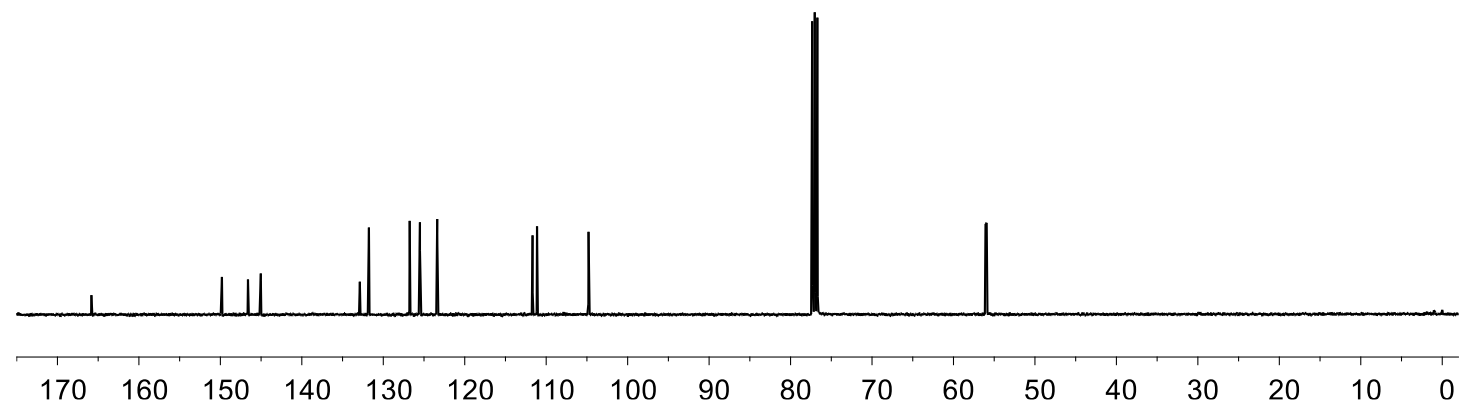



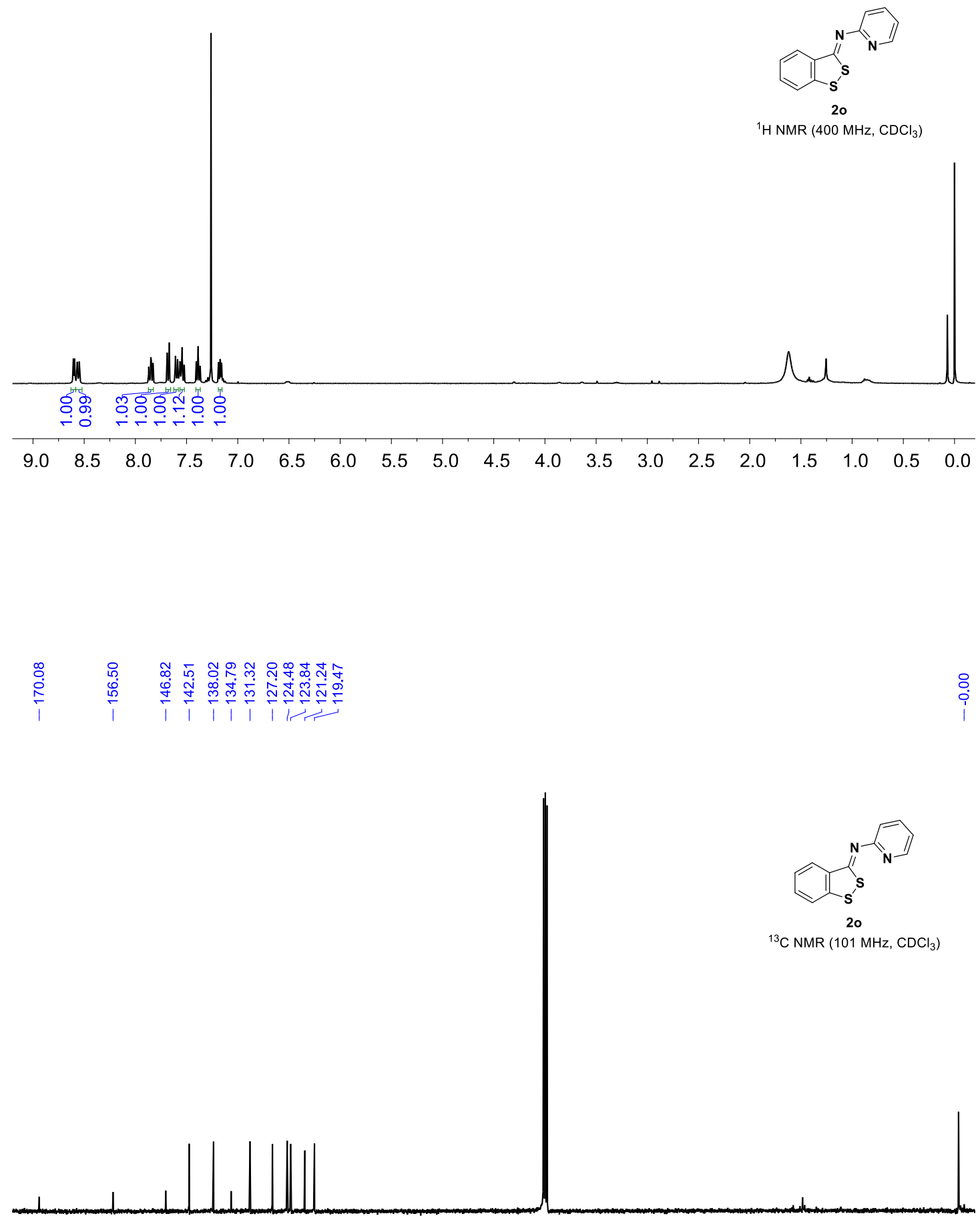

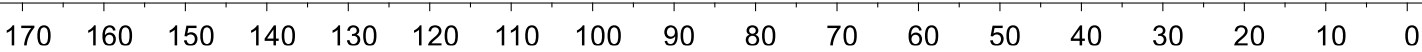




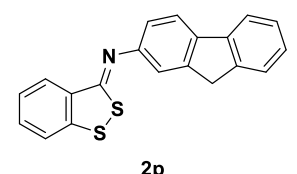

${ }^{1} \mathrm{H} \mathrm{NMR}\left(400 \mathrm{MHz}, \mathrm{CDCl}_{3}\right)$

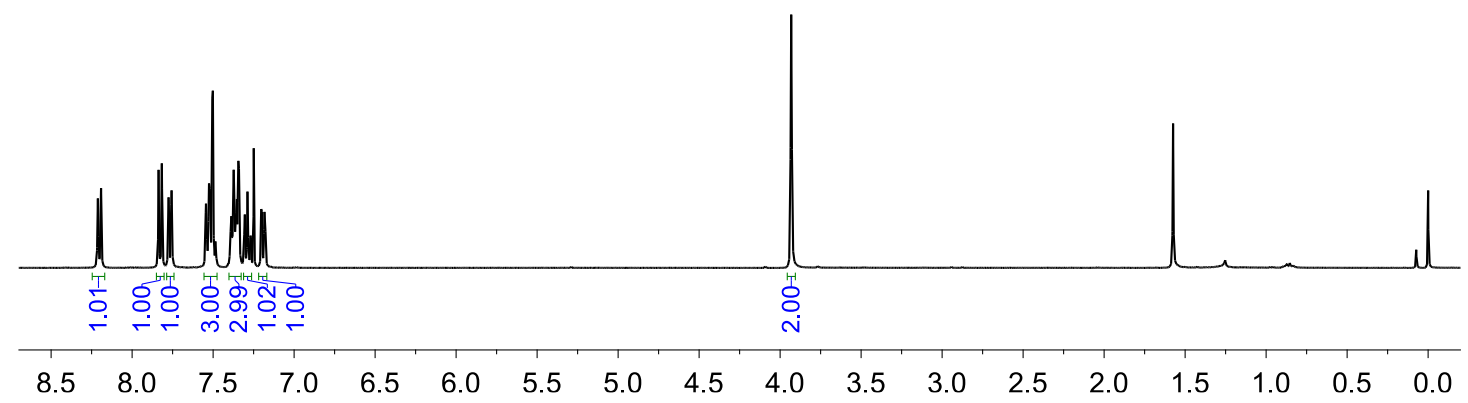

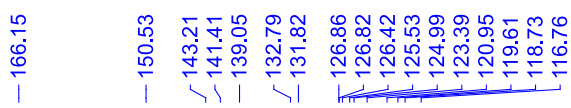

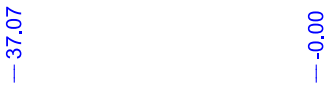

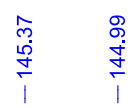
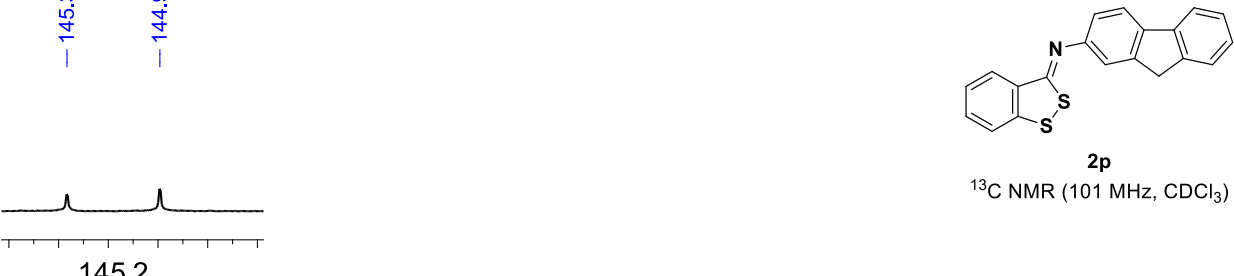

${ }^{13} \mathrm{C} \mathrm{NMR}\left(101 \mathrm{MHz}, \mathrm{CDCl}_{3}\right)$

145.2

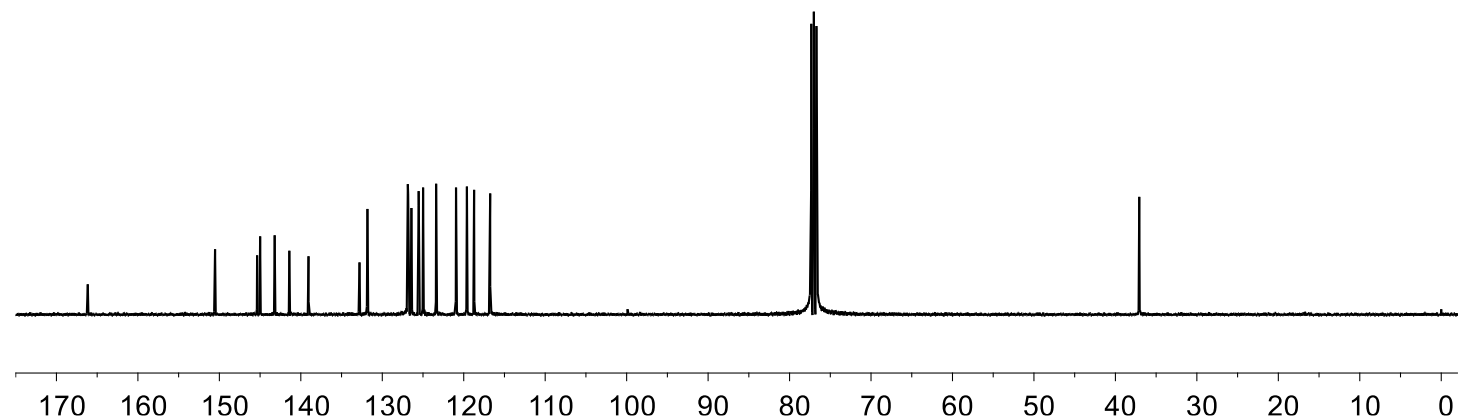




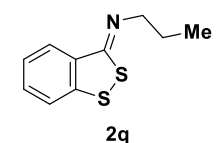

${ }^{1} \mathrm{H} \mathrm{NMR}\left(400 \mathrm{MHz}, \mathrm{CDCl}_{3}\right)$

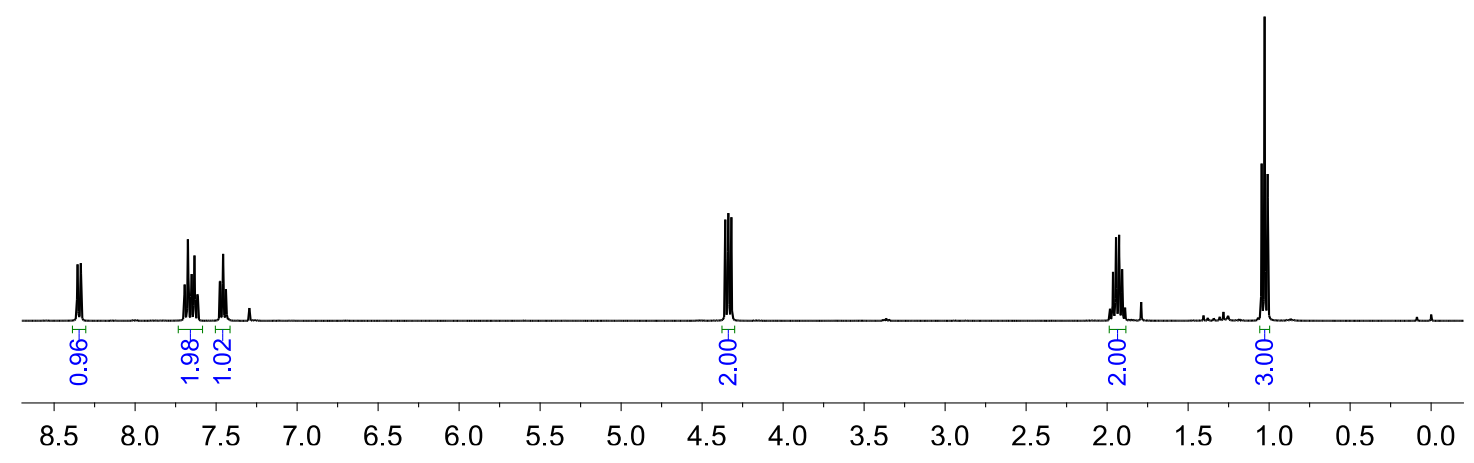

$\begin{array}{ll}0 & \\ \infty & 1\end{array}$

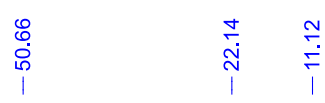

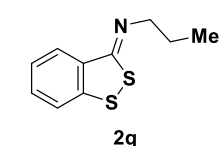

${ }^{13} \mathrm{C} \mathrm{NMR}\left(101 \mathrm{MHz}, \mathrm{CDCl}_{3}\right)$

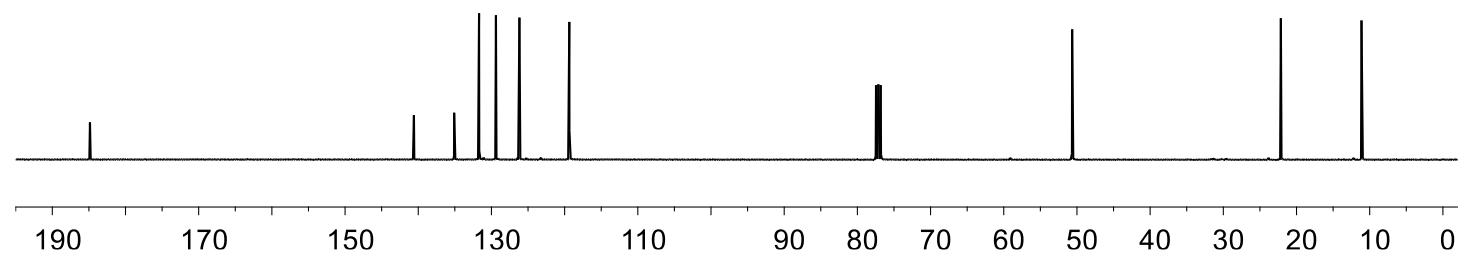




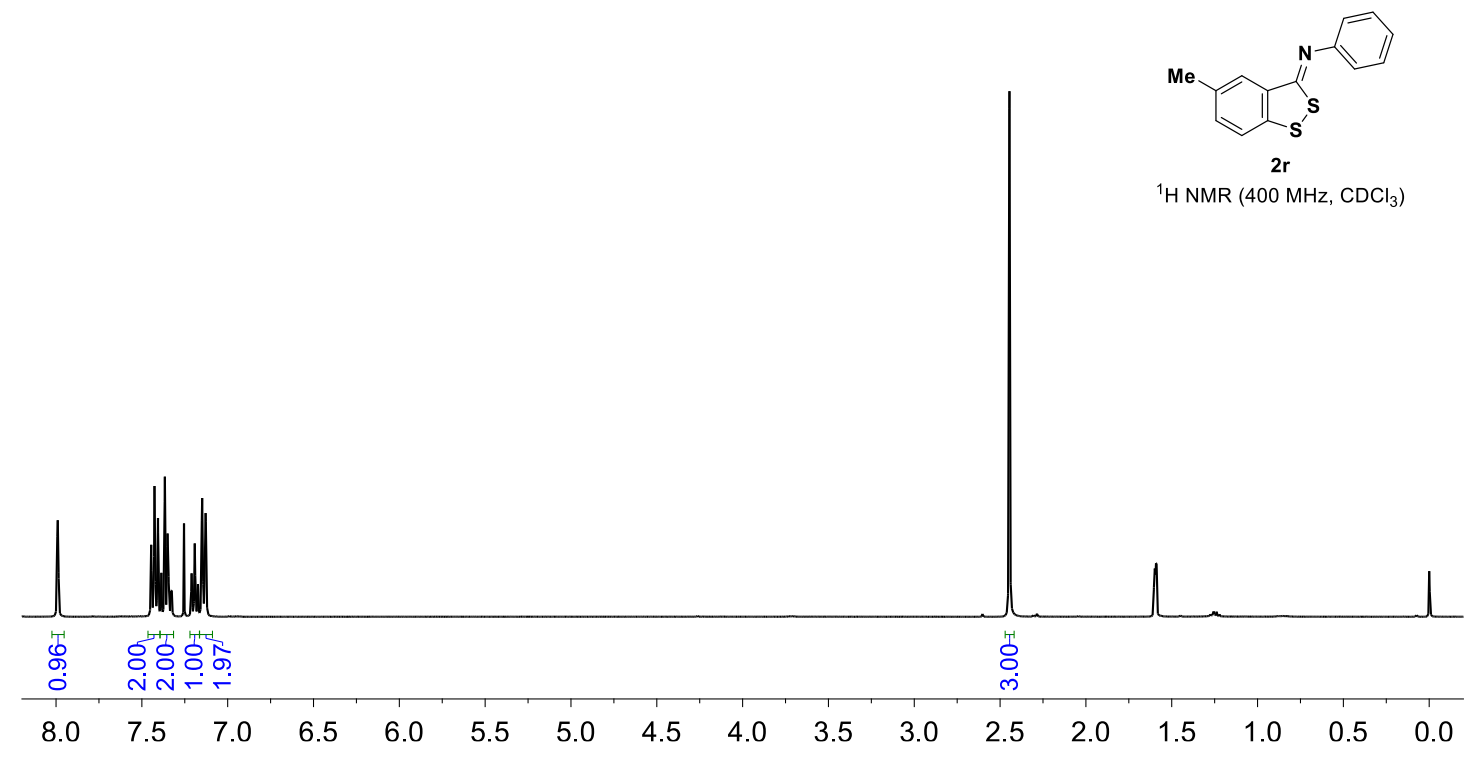

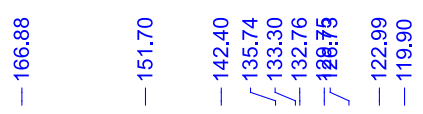

$\stackrel{\stackrel{\infty}{D}}{\stackrel{\infty}{\sim}}$
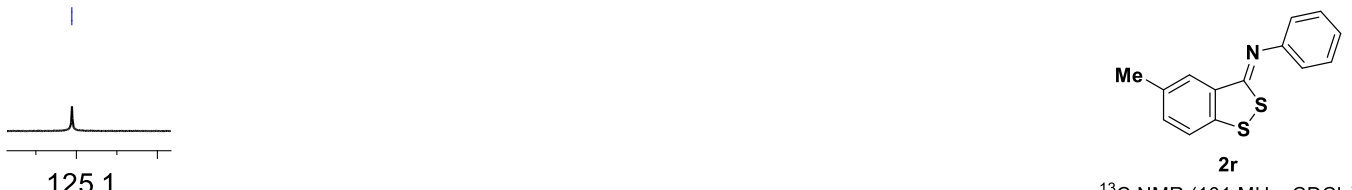

${ }^{13} \mathrm{CNMR}\left(101 \mathrm{MHz}, \mathrm{CDCl}_{3}\right)$

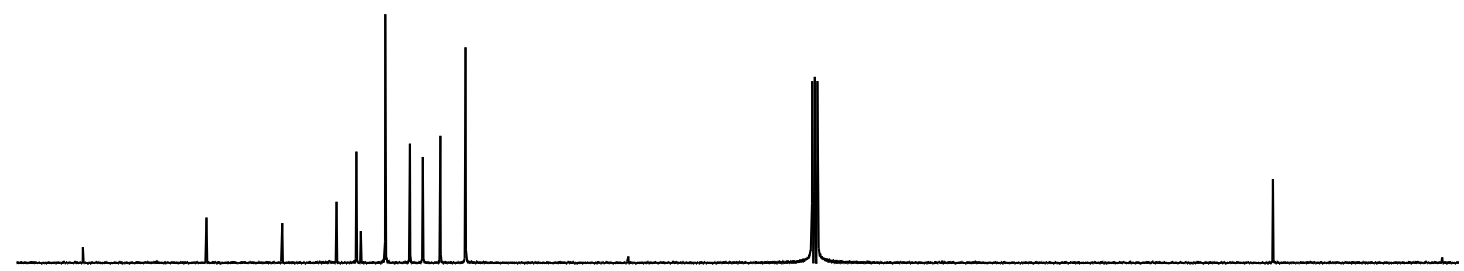

$\begin{array}{llllllllllllllllll}170 & 160 & 150 & 140 & 130 & 120 & 110 & 100 & 90 & 80 & 70 & 60 & 50 & 40 & 30 & 20 & 10 & 0\end{array}$ 


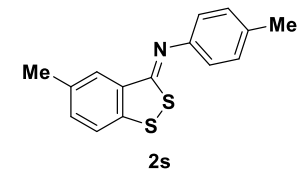

${ }^{1} \mathrm{H} \mathrm{NMR}\left(400 \mathrm{MHz}, \mathrm{CDCl}_{3}\right)$

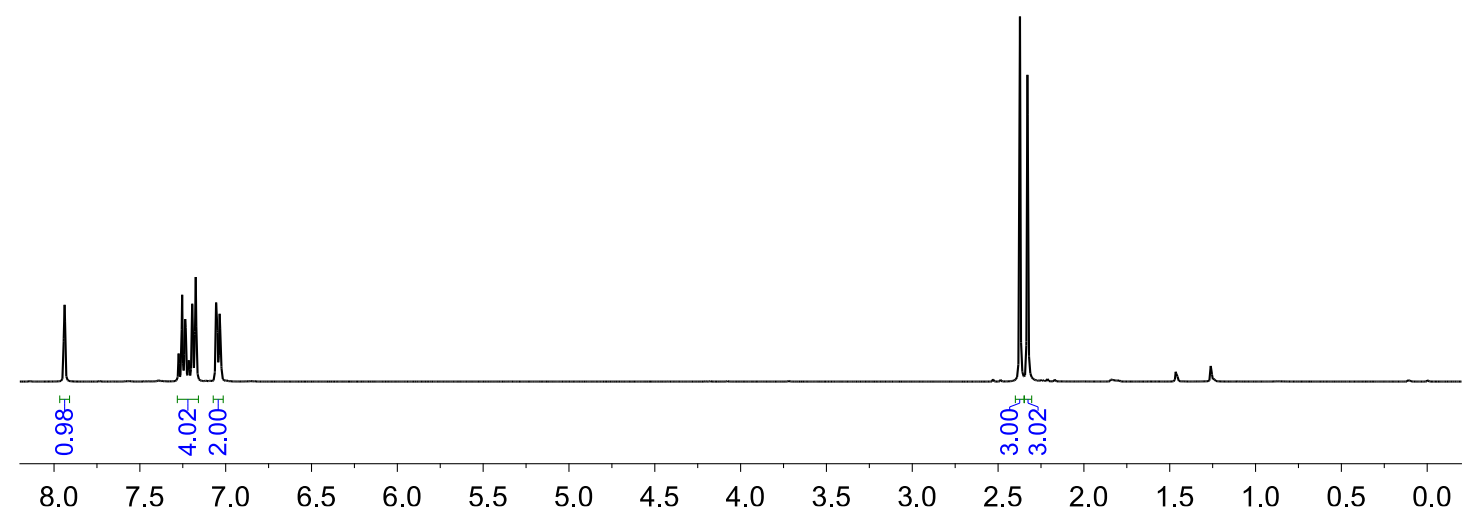

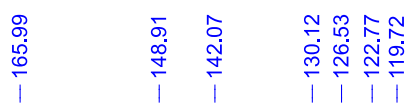
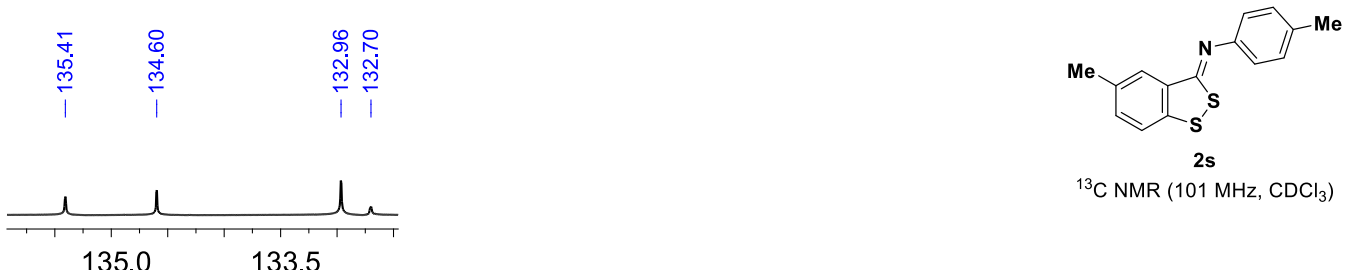

${ }^{13} \mathrm{C}$ NMR $\left(101 \mathrm{MHz}, \mathrm{CDCl}_{3}\right)$

$135.0 \quad 133.5$

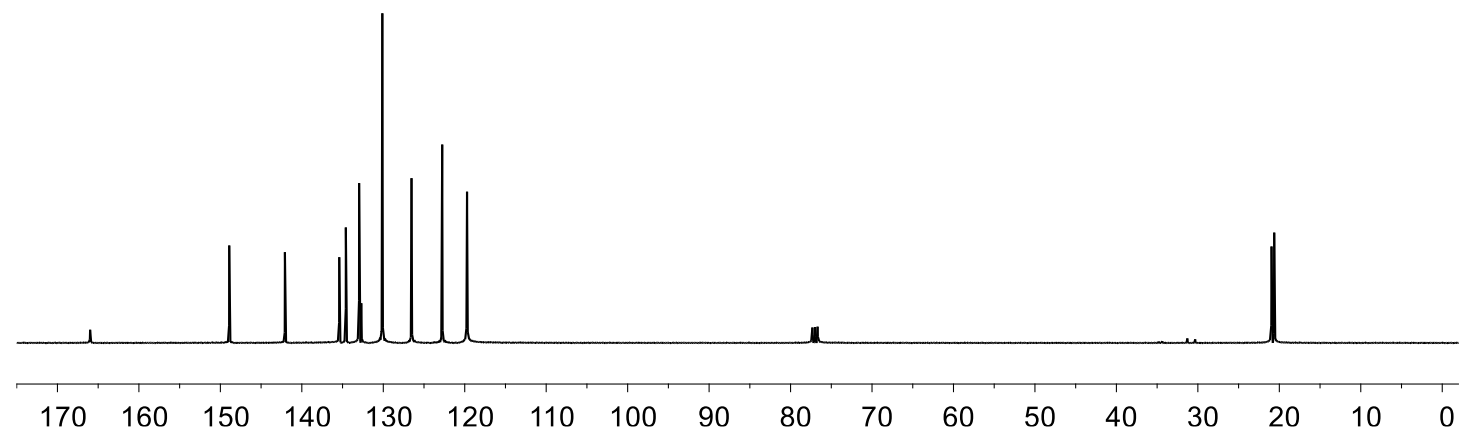




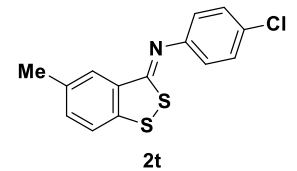

${ }^{1} \mathrm{H} \mathrm{NMR}\left(400 \mathrm{MHz}, \mathrm{CDCl}_{3}\right)$
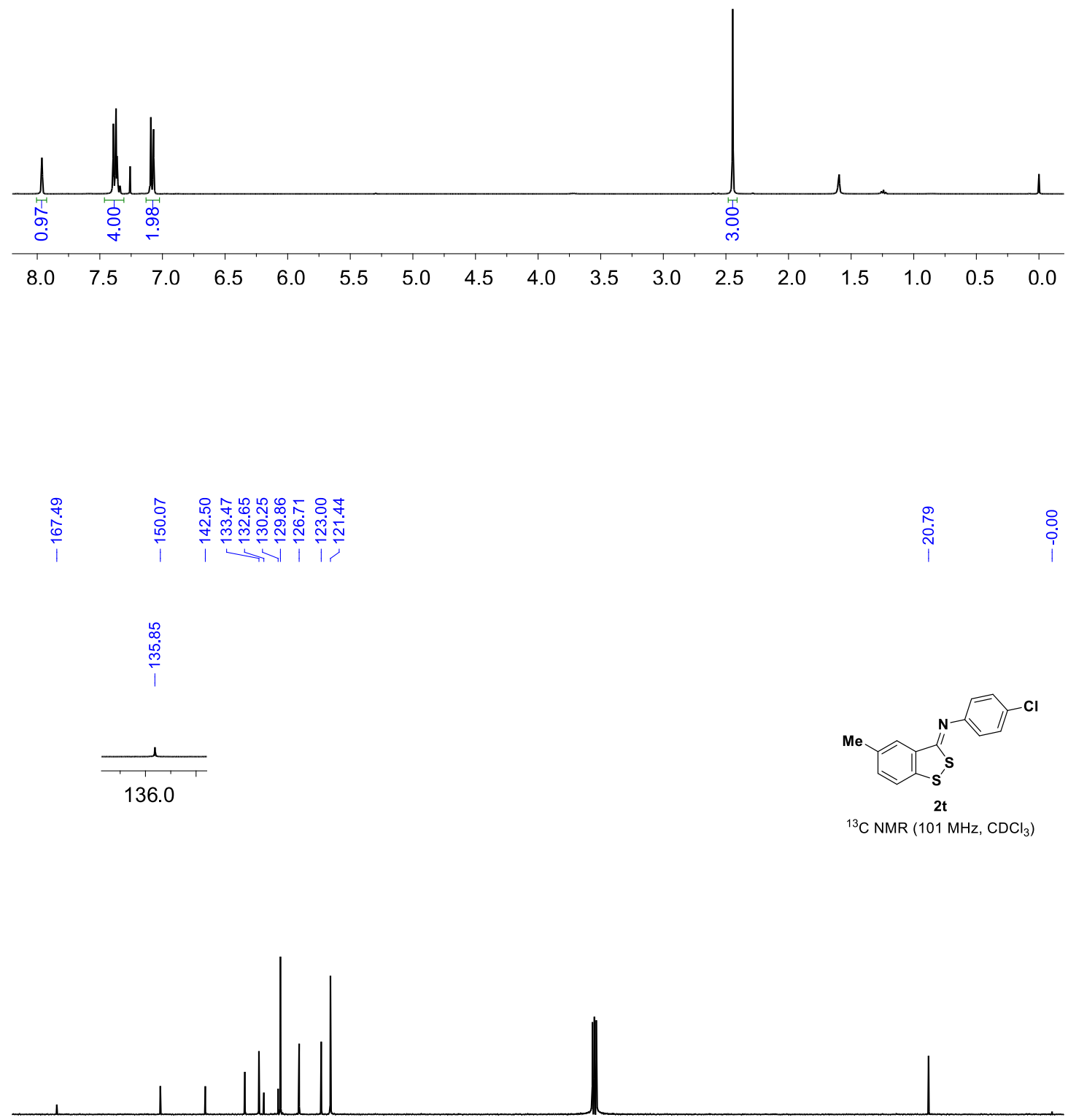

$\begin{array}{llllllllllllllllll}170 & 160 & 150 & 140 & 130 & 120 & 110 & 100 & 90 & 80 & 70 & 60 & 50 & 40 & 30 & 20 & 10 & 0\end{array}$ 

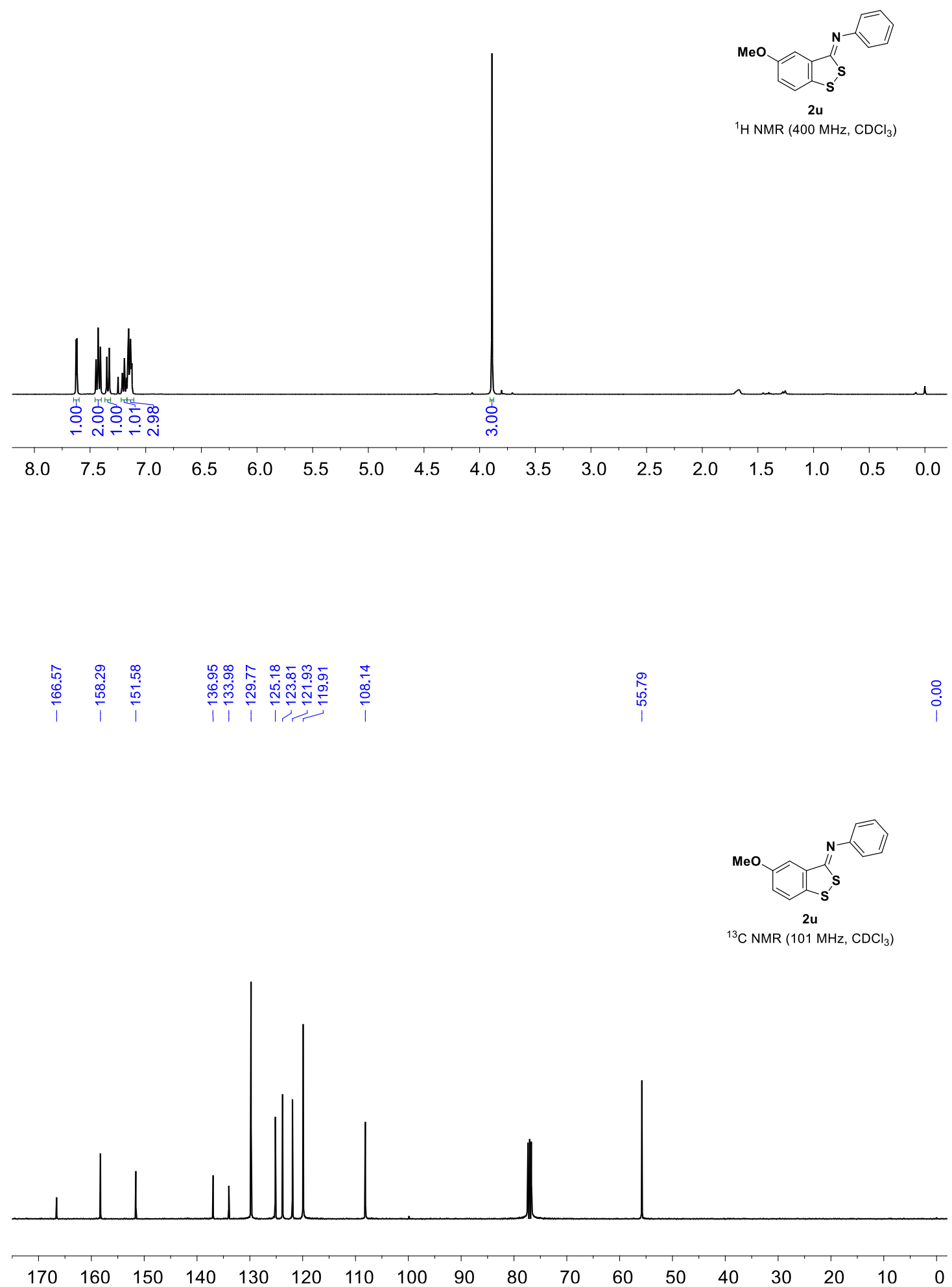

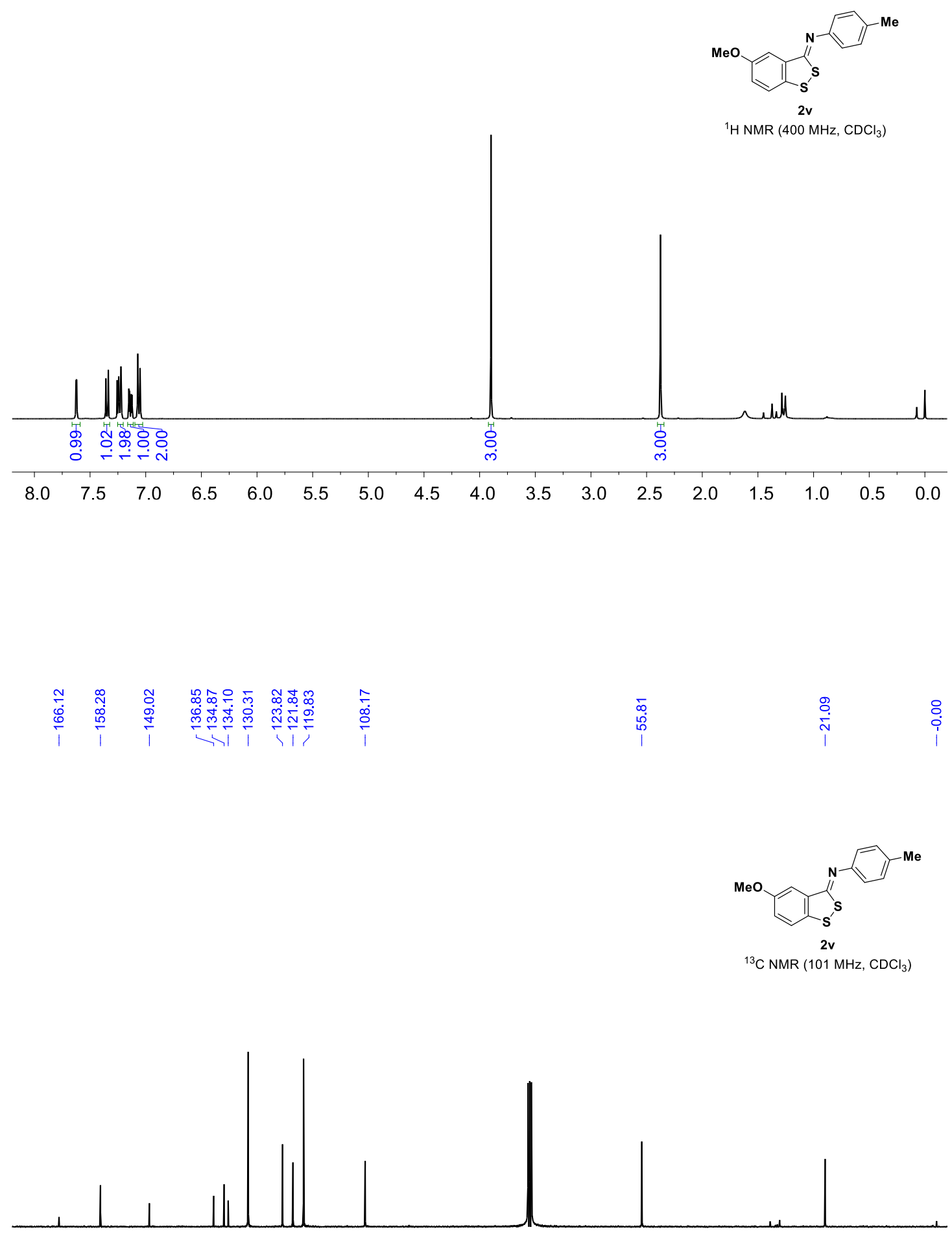

$\begin{array}{lll}170 & 160 & 150\end{array}$

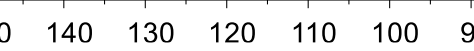

$80 \quad 70 \quad 60$

$60 \quad 50 \quad 40$

$\begin{array}{lllll}40 & 30 & 20 & 10 & 0\end{array}$ 


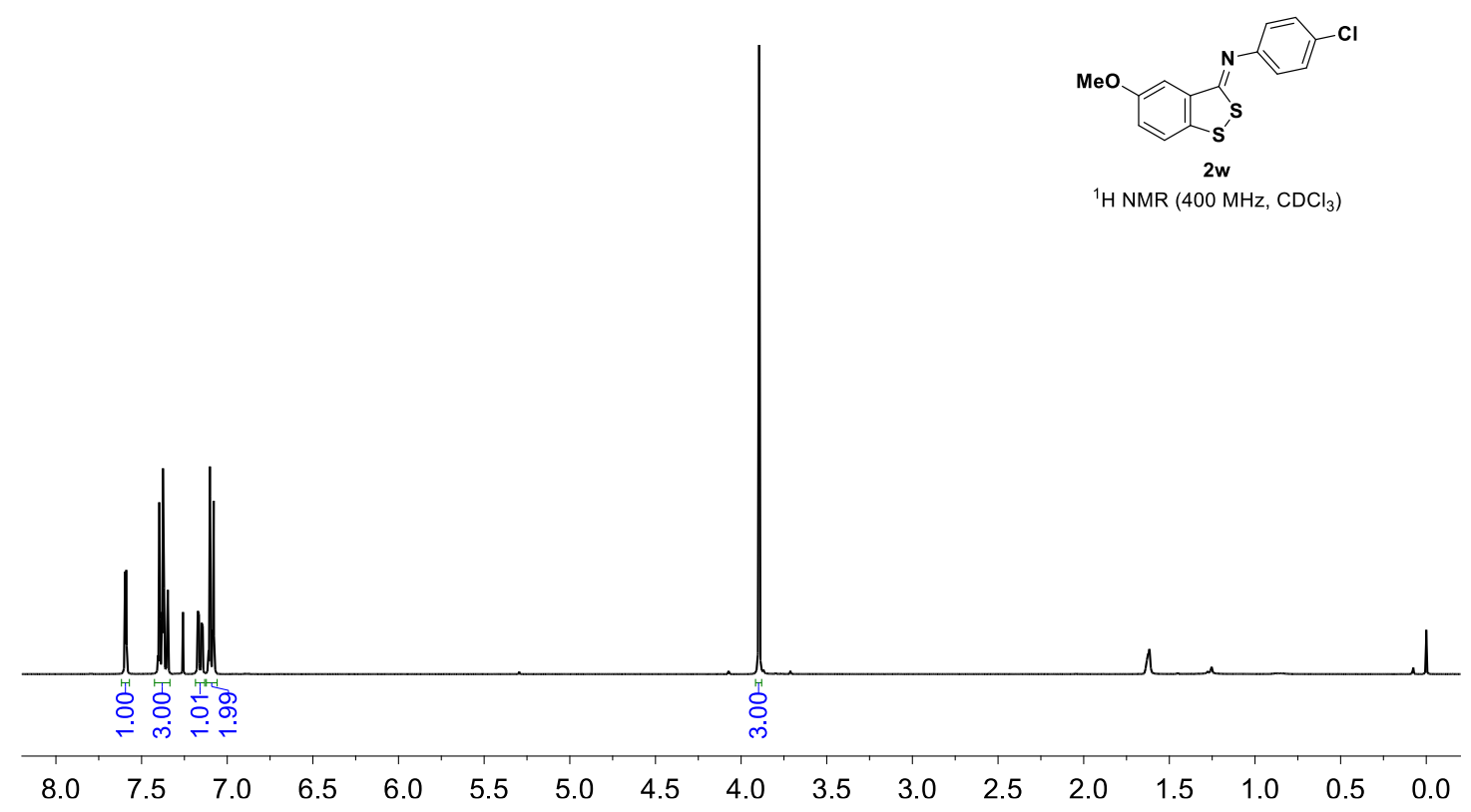

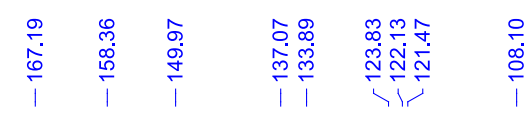

0
$\infty$
100
10
1
1

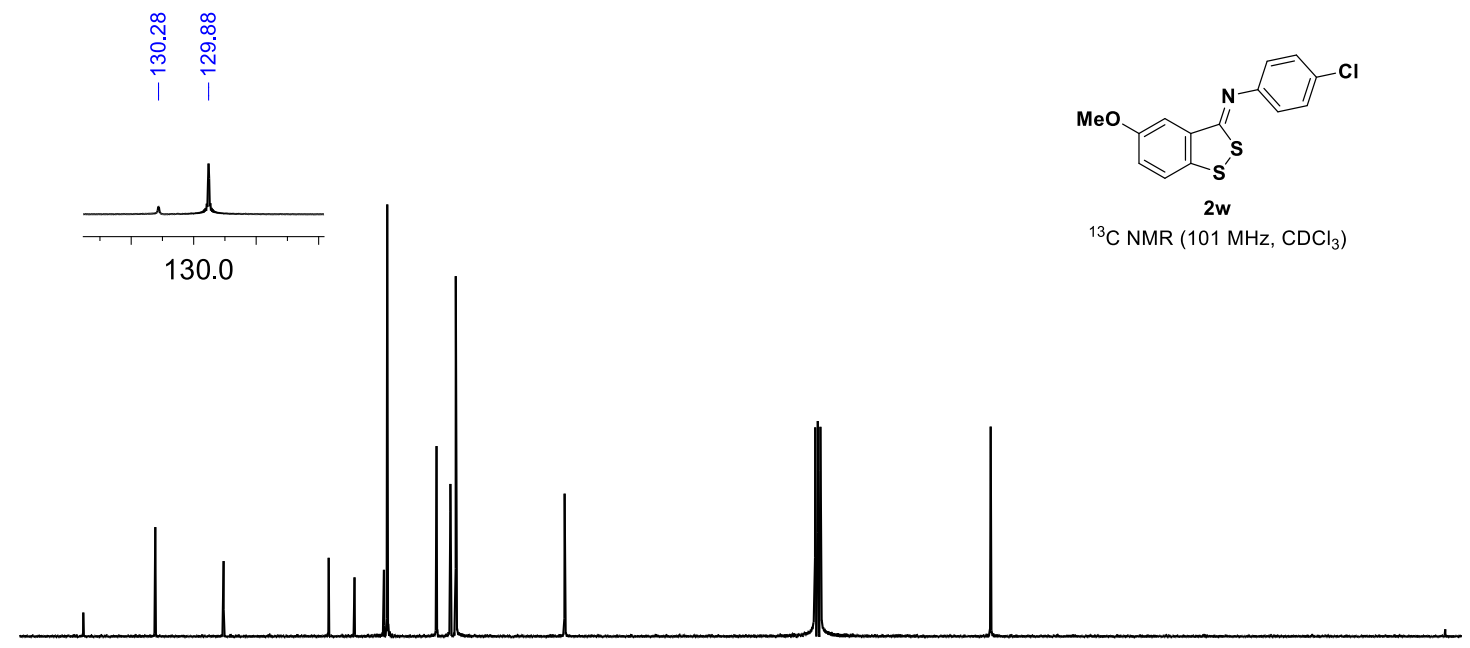

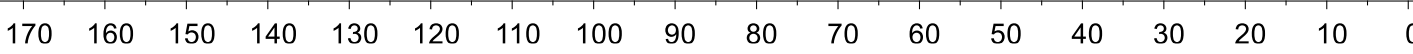




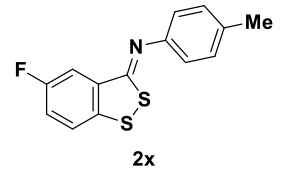

${ }^{1} \mathrm{H}$ NMR $\left(400 \mathrm{MHz}, \mathrm{CDCl}_{3}\right)$
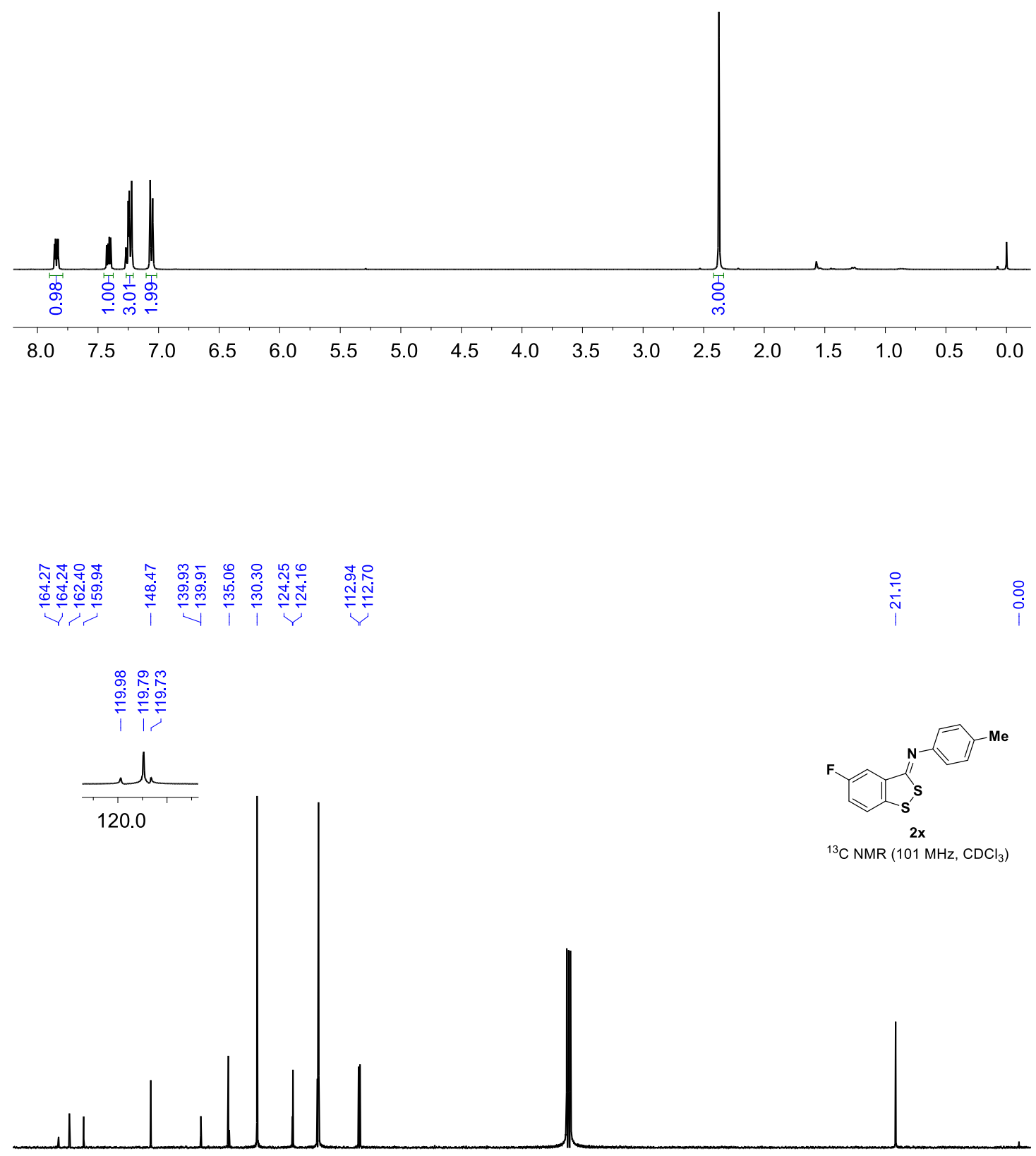

170

$160 \quad 150$

$130 \quad 12$

$\begin{array}{lllll}110 & 100 & 90 & 80\end{array}$

$\begin{array}{llllllll}70 & 60 & 50 & 40 & 30 & 20 & 10 & 0\end{array}$ 


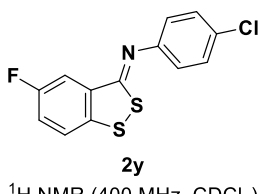

${ }^{1} \mathrm{HNMR}\left(400 \mathrm{MHz}, \mathrm{CDCl}_{3}\right)$

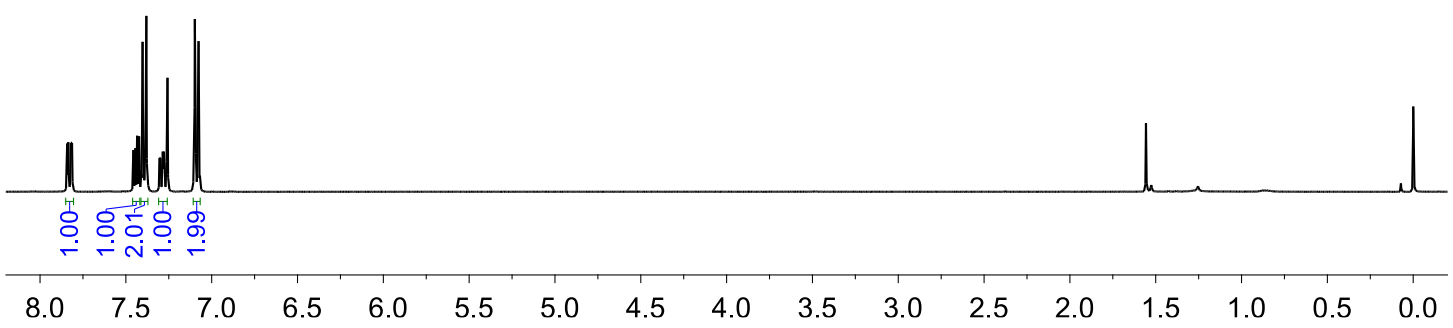

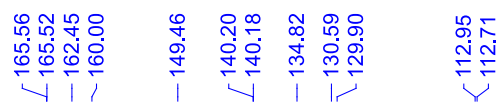
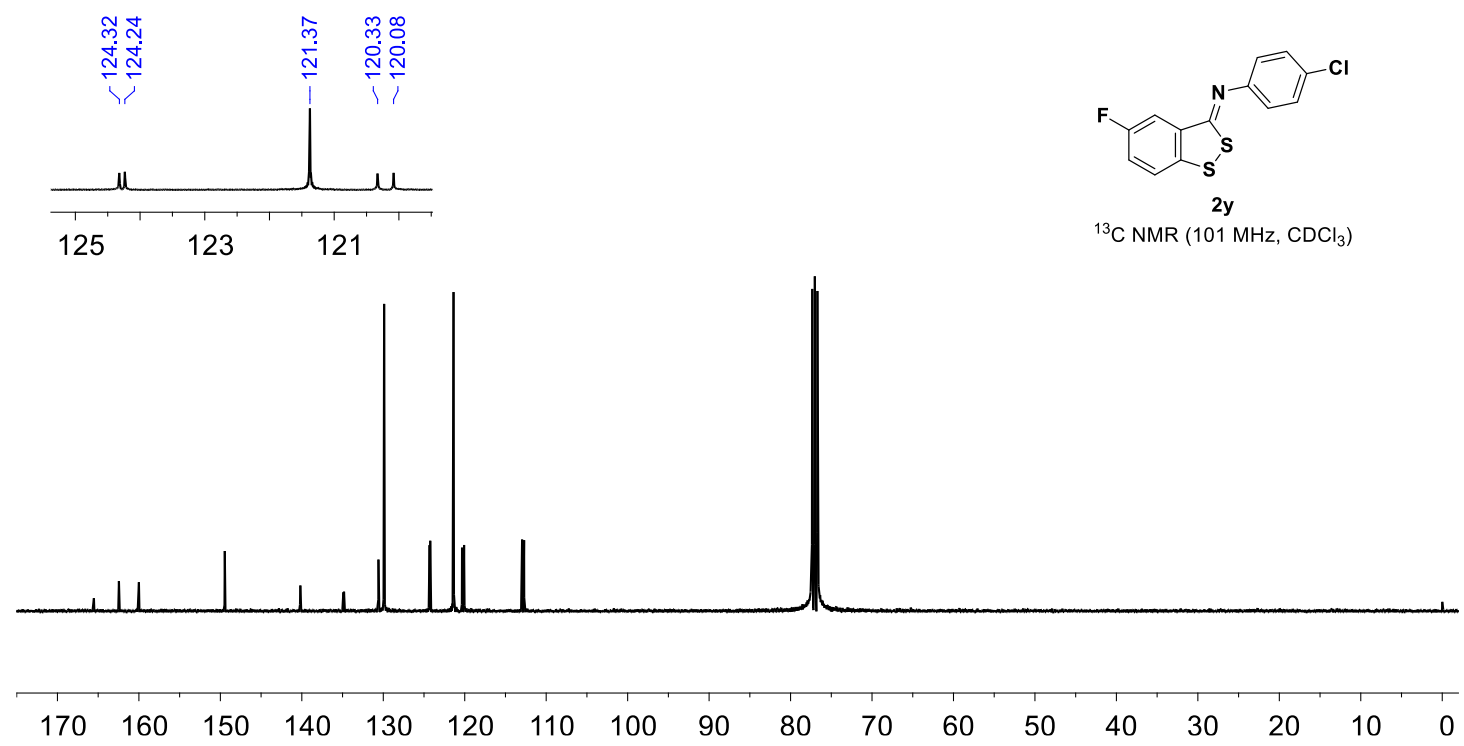


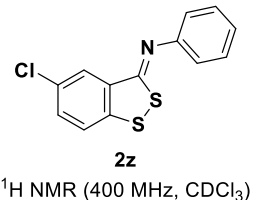

${ }^{1} \mathrm{H}$ NMR $\left(400 \mathrm{MHz}, \mathrm{CDCl}_{3}\right)$

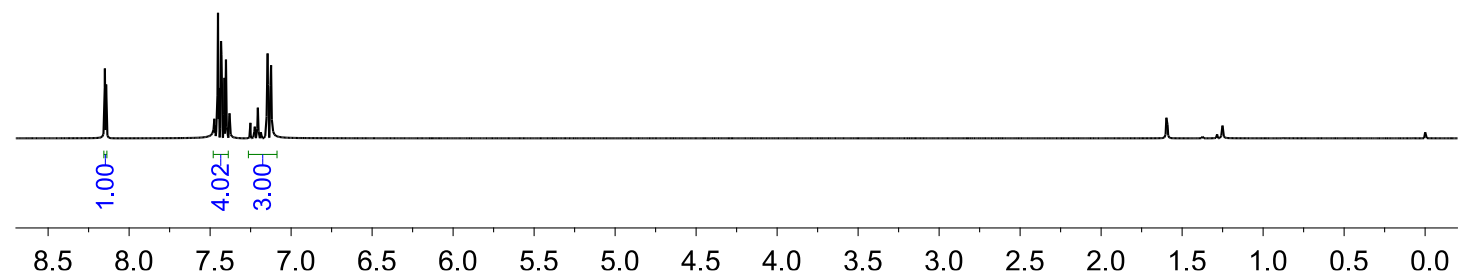

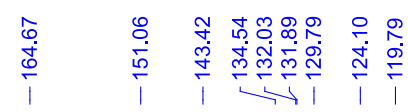
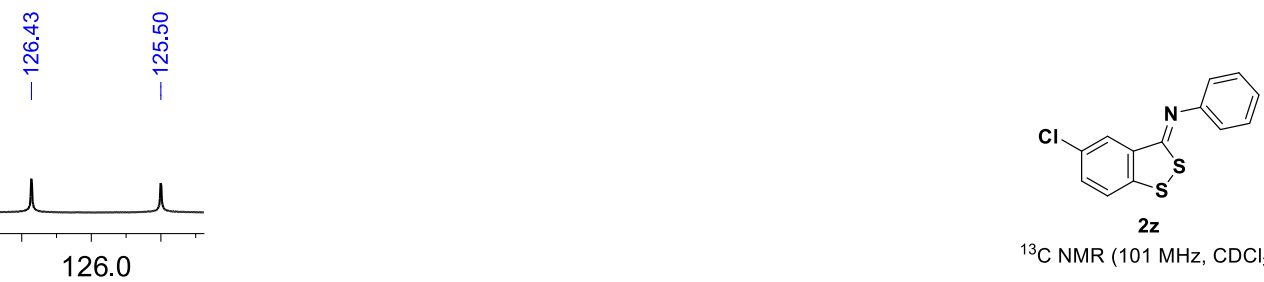

${ }^{13} \mathrm{C} \mathrm{NMR}\left(101 \mathrm{MHz}, \mathrm{CDCl}_{3}\right)$

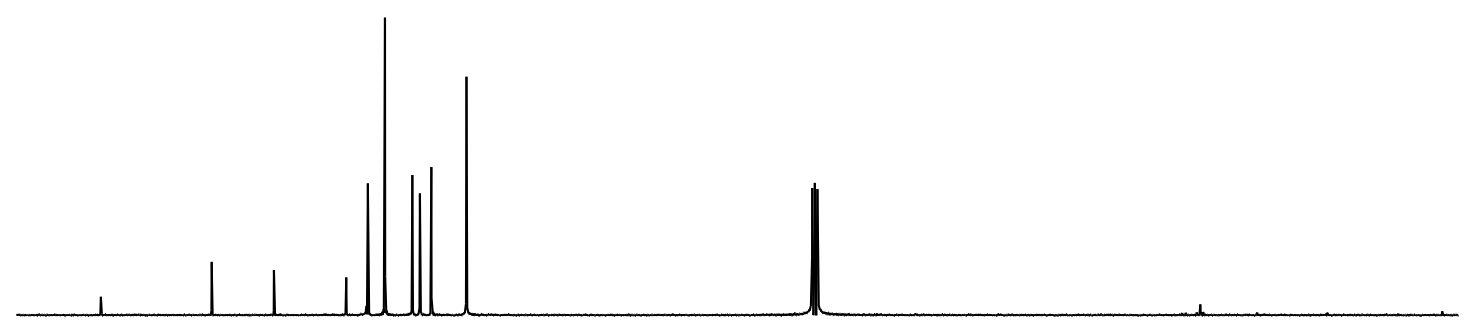

$\begin{array}{llllllllllllllllll}170 & 160 & 150 & 140 & 130 & 120 & 110 & 100 & 90 & 80 & 70 & 60 & 50 & 40 & 30 & 20 & 10 & 0\end{array}$ 


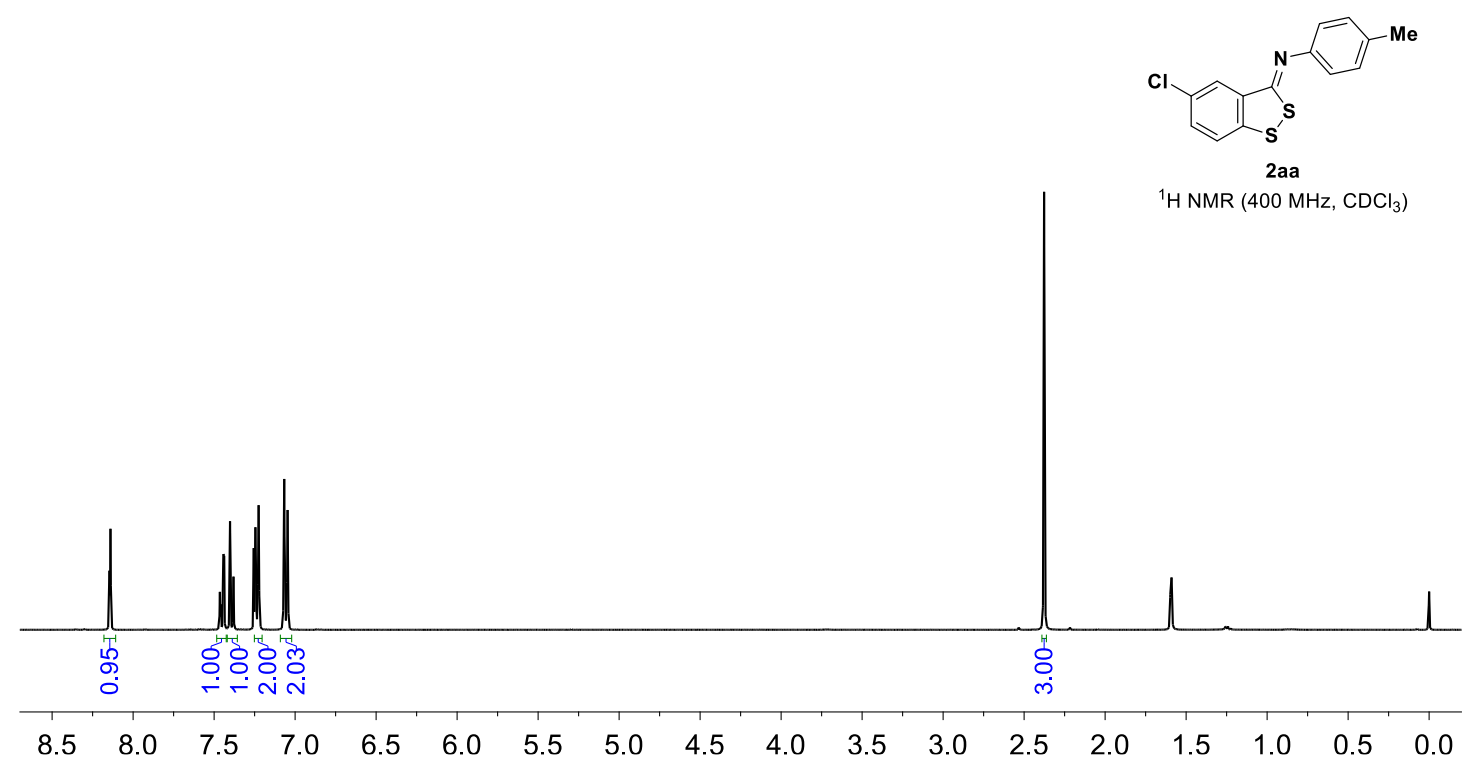

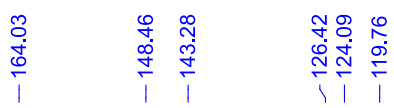

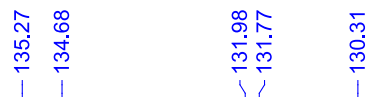
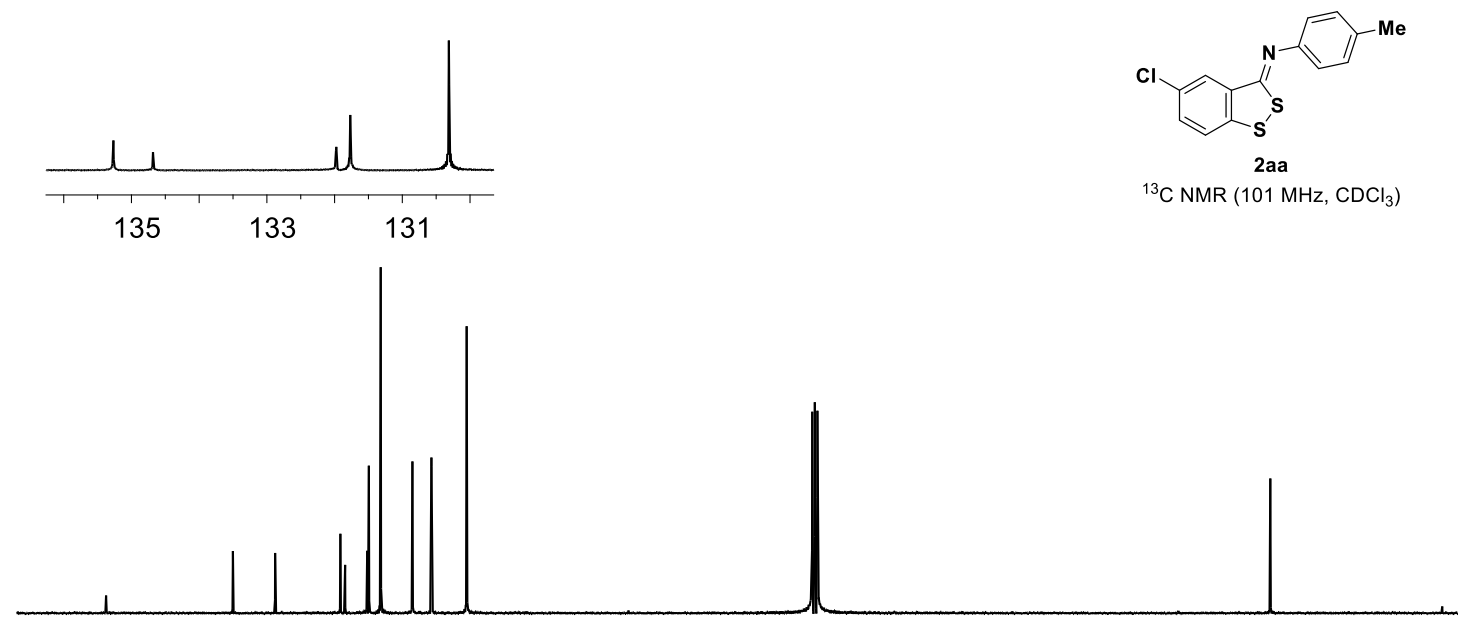

$\begin{array}{llllllllllllllllll}170 & 160 & 150 & 140 & 130 & 120 & 110 & 100 & 90 & 80 & 70 & 60 & 50 & 40 & 30 & 20 & 10 & 0\end{array}$ 


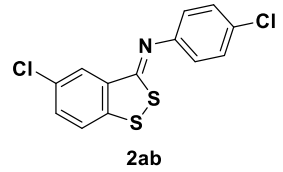

${ }^{1} \mathrm{H} \mathrm{NMR}\left(400 \mathrm{MHz}, \mathrm{CDCl}_{3}\right)$

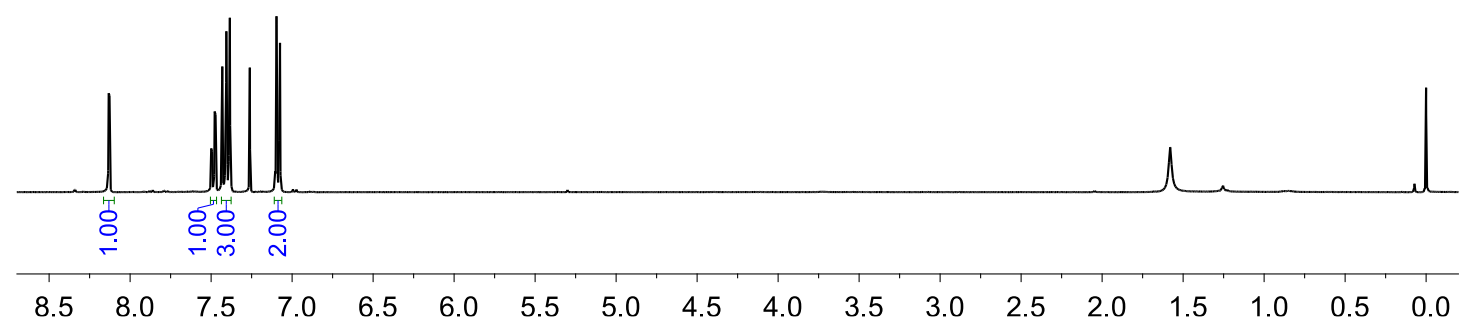

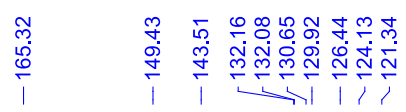

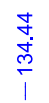
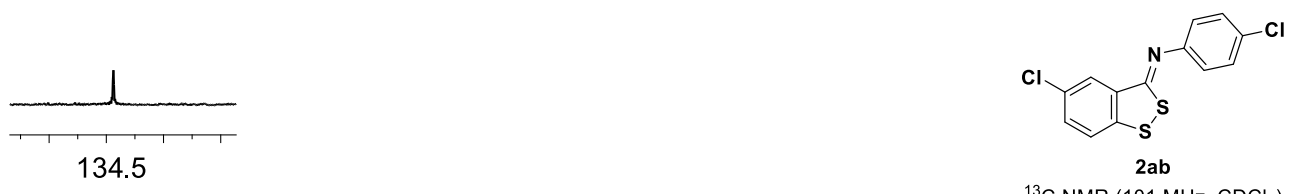

${ }^{13} \mathrm{C}$ NMR $\left(101 \mathrm{MHz}, \mathrm{CDCl}_{3}\right)$

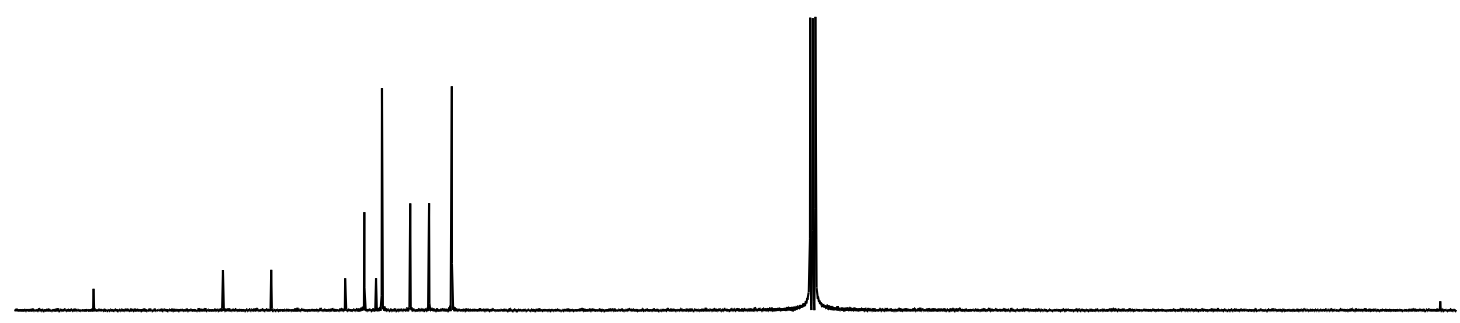

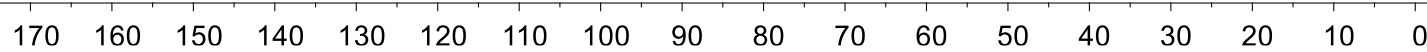




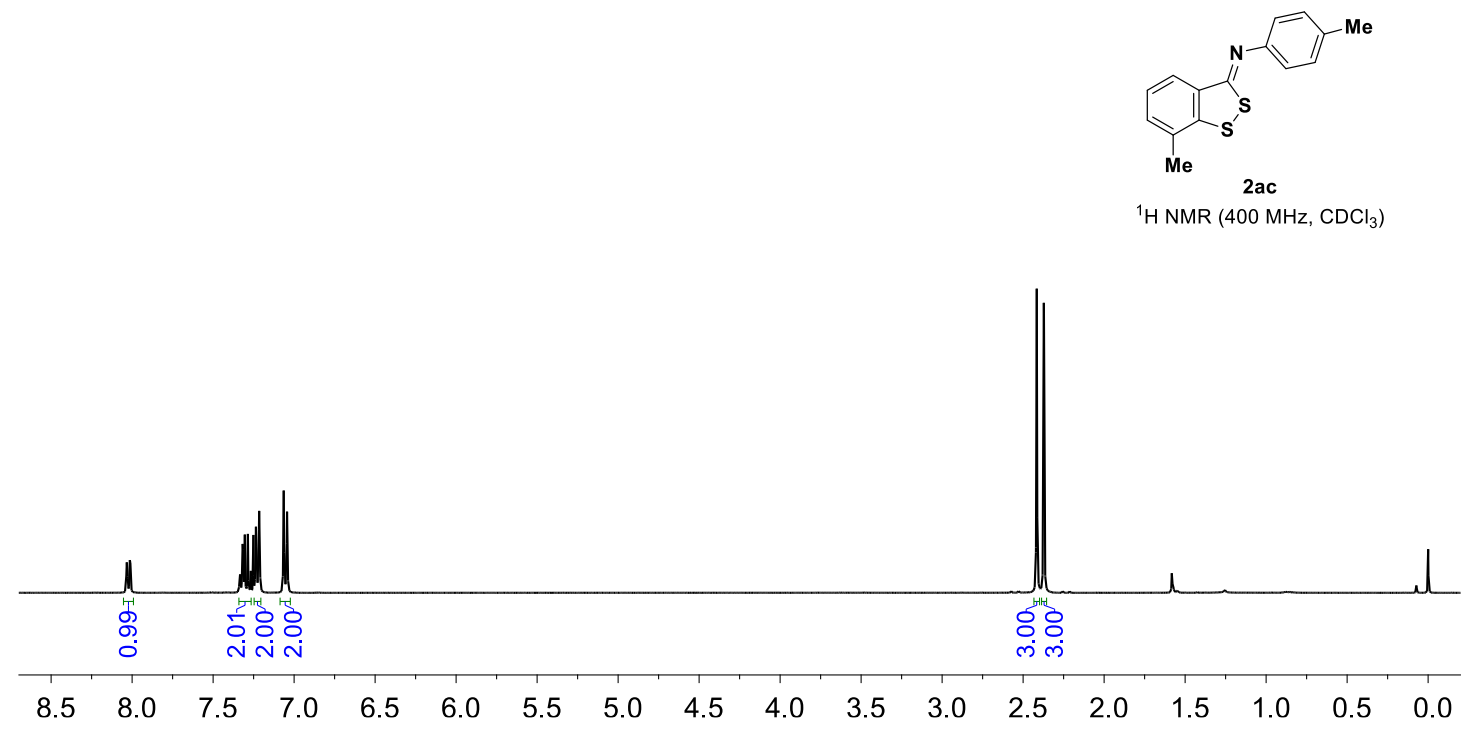

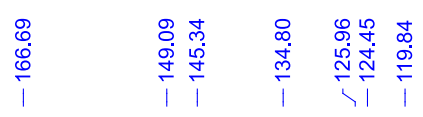
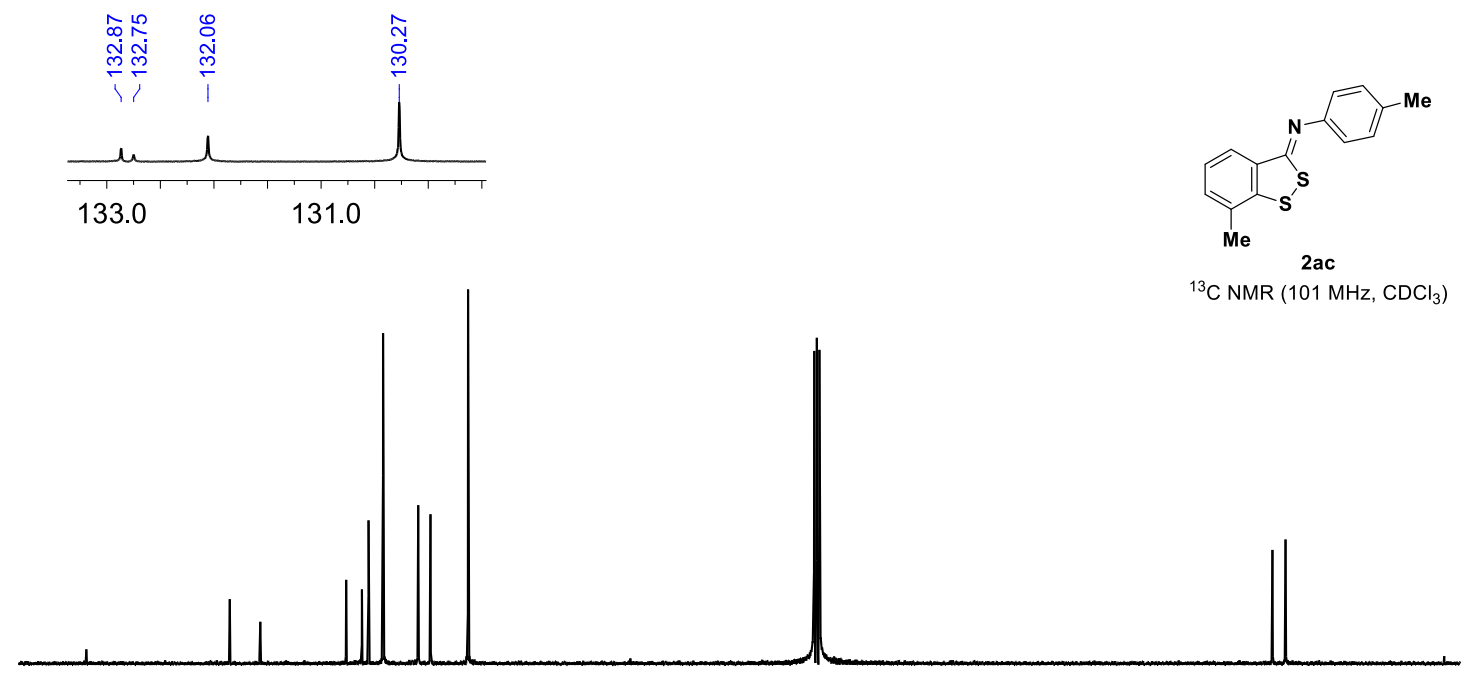

$\begin{array}{llllllllllllllllll}170 & 160 & 150 & 140 & 130 & 120 & 110 & 100 & 90 & 80 & 70 & 60 & 50 & 40 & 30 & 20 & 10 & 0\end{array}$ 


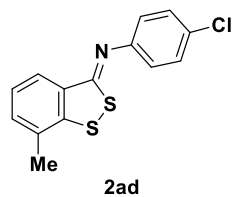

${ }^{1} \mathrm{H} \mathrm{NMR}\left(400 \mathrm{MHz}, \mathrm{CDCl}_{3}\right)$

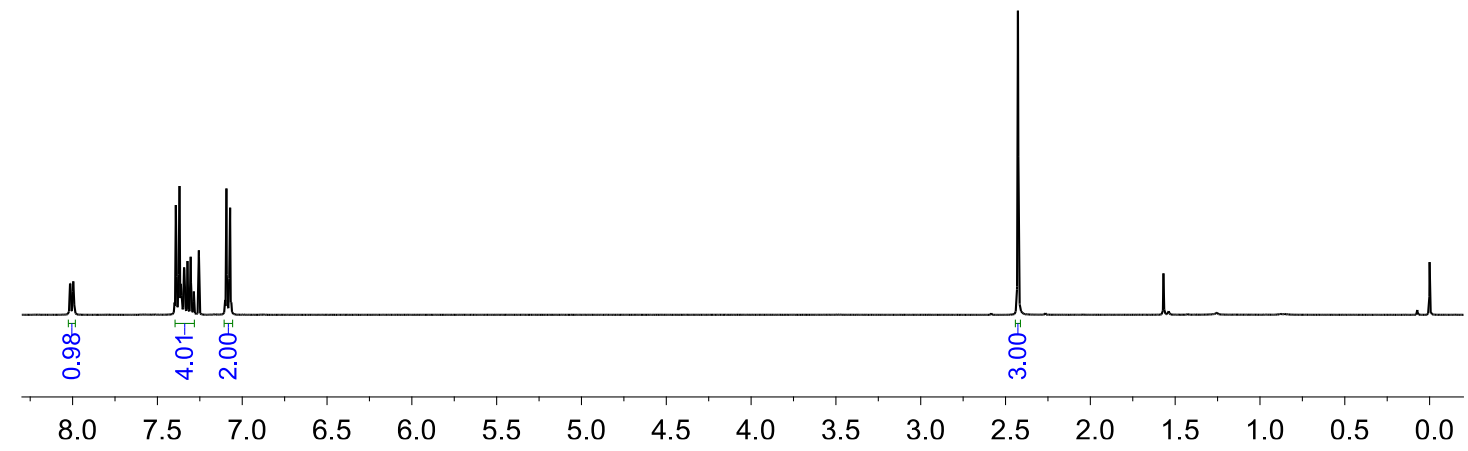

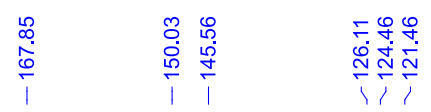
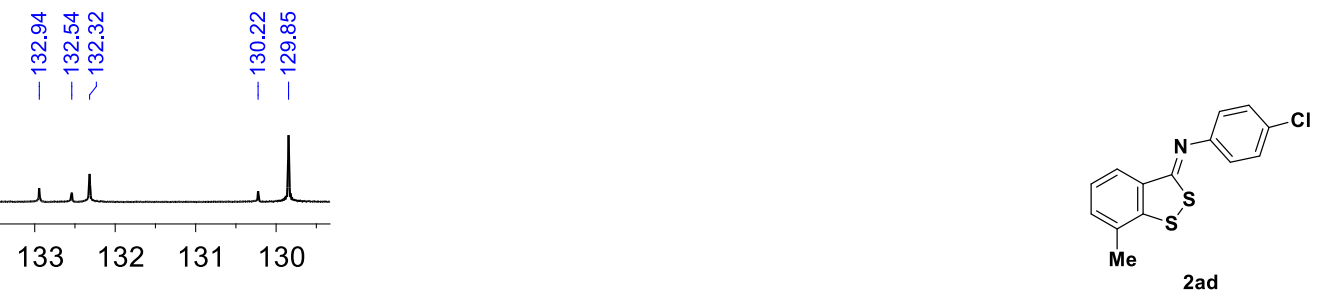

${ }^{13} \mathrm{C}$ NMR $\left(101 \mathrm{MHz}, \mathrm{CDCl}_{3}\right)$

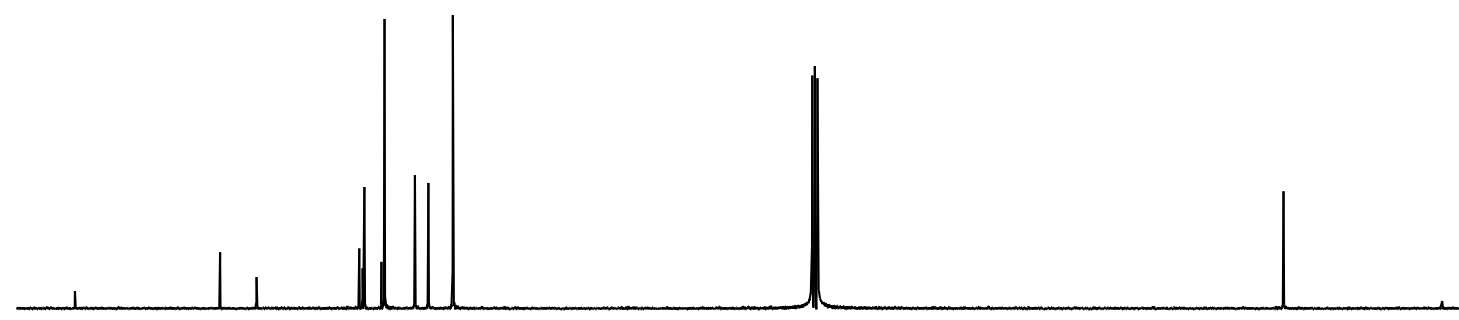

$\begin{array}{llllllllllllllllll}170 & 160 & 150 & 140 & 130 & 120 & 110 & 100 & 90 & 80 & 70 & 60 & 50 & 40 & 30 & 20 & 10 & 0\end{array}$ 


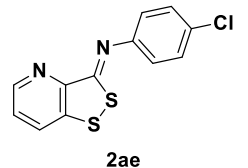

${ }^{1} \mathrm{H} \mathrm{NMR}\left(400 \mathrm{MHz}, \mathrm{CDCl}_{3}\right)$

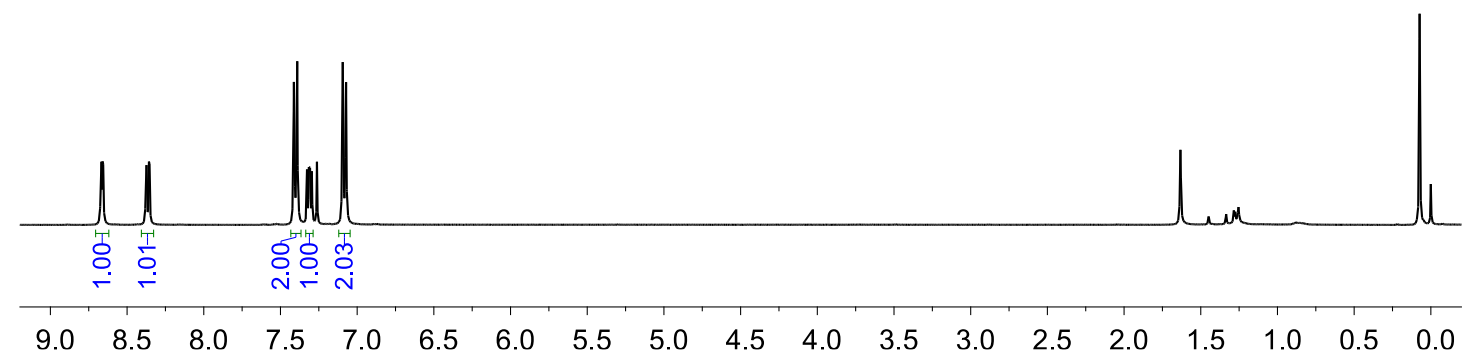

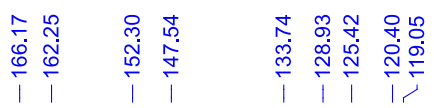
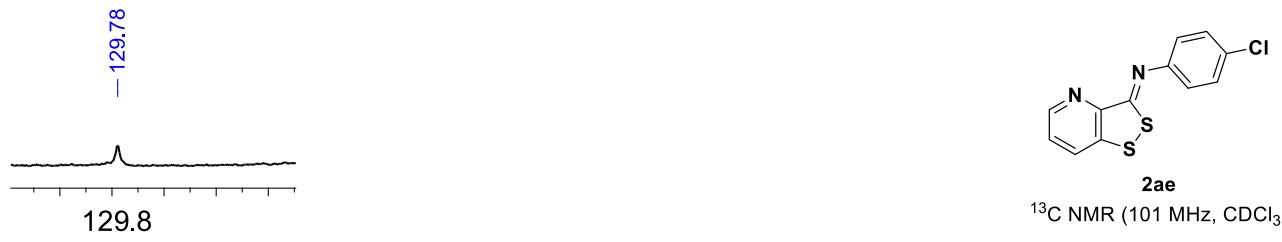

${ }^{13} \mathrm{C} \mathrm{NMR}\left(101 \mathrm{MHz}, \mathrm{CDCl}_{3}\right)$

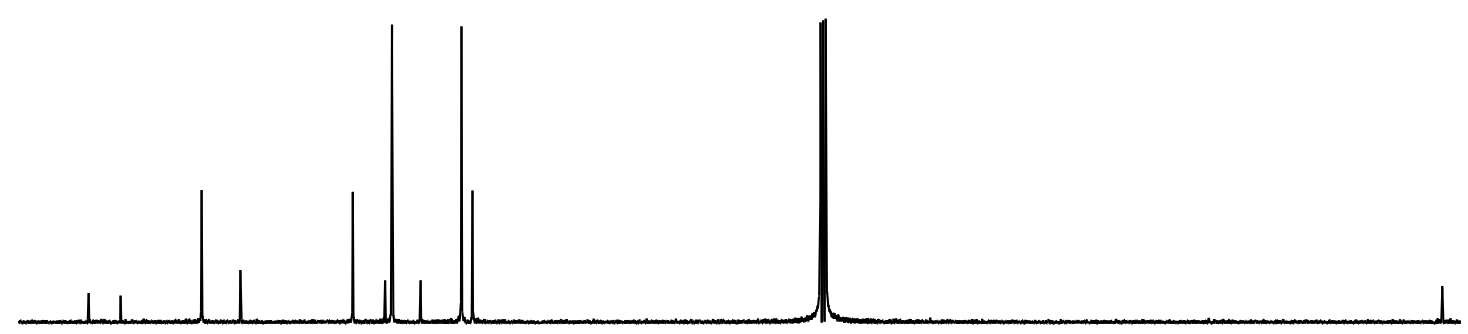

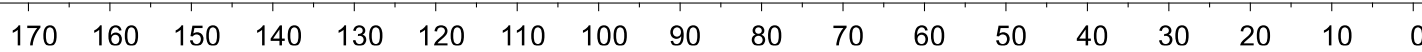




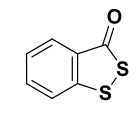

$3 a$

${ }^{1} \mathrm{H}$ NMR $\left(400 \mathrm{MHz}, \mathrm{CDCl}_{3}\right)$
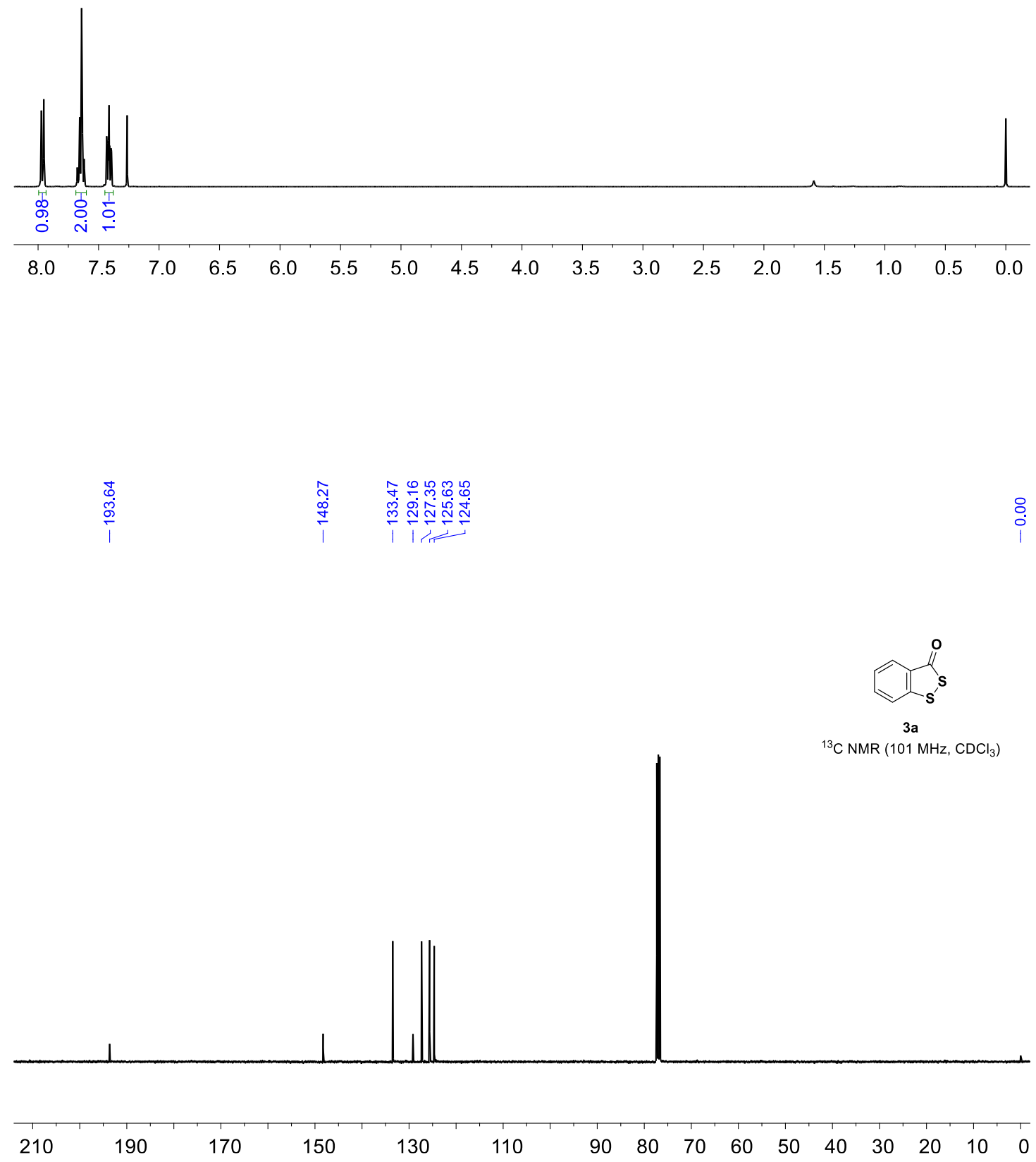


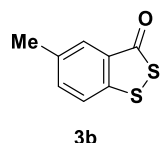

${ }^{1} \mathrm{H}$ NMR $\left(400 \mathrm{MHz}, \mathrm{CDCl}_{3}\right)$
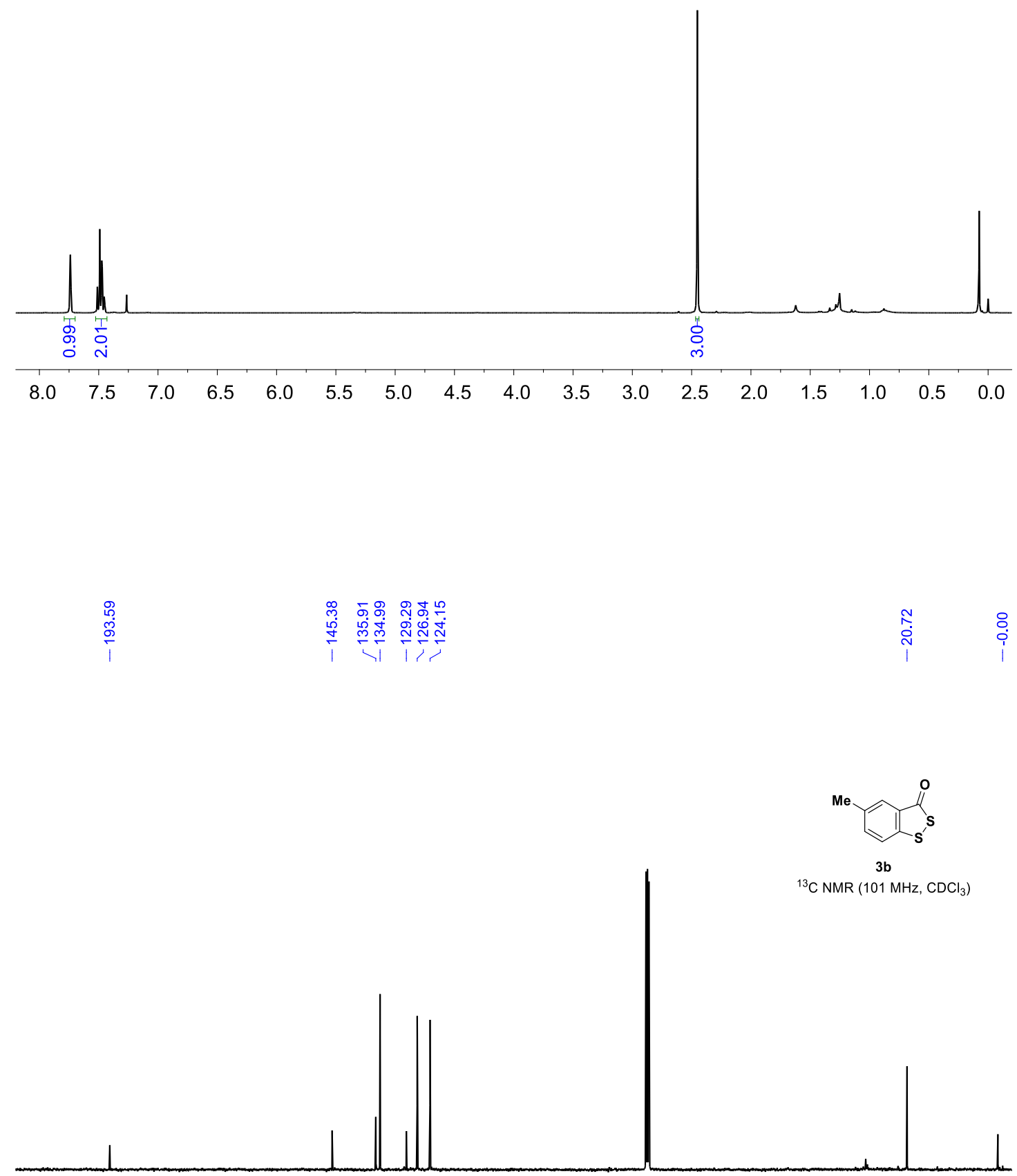

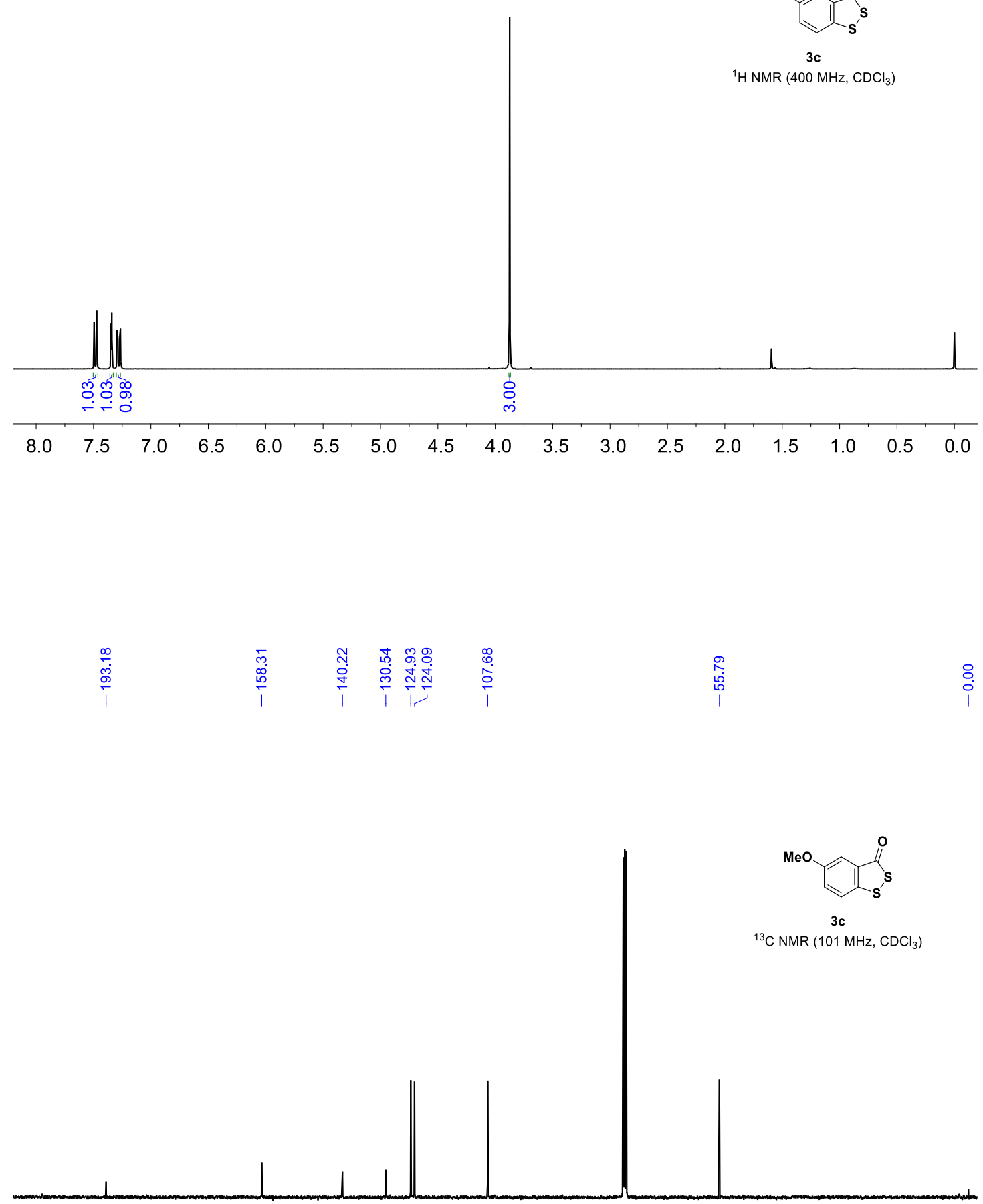

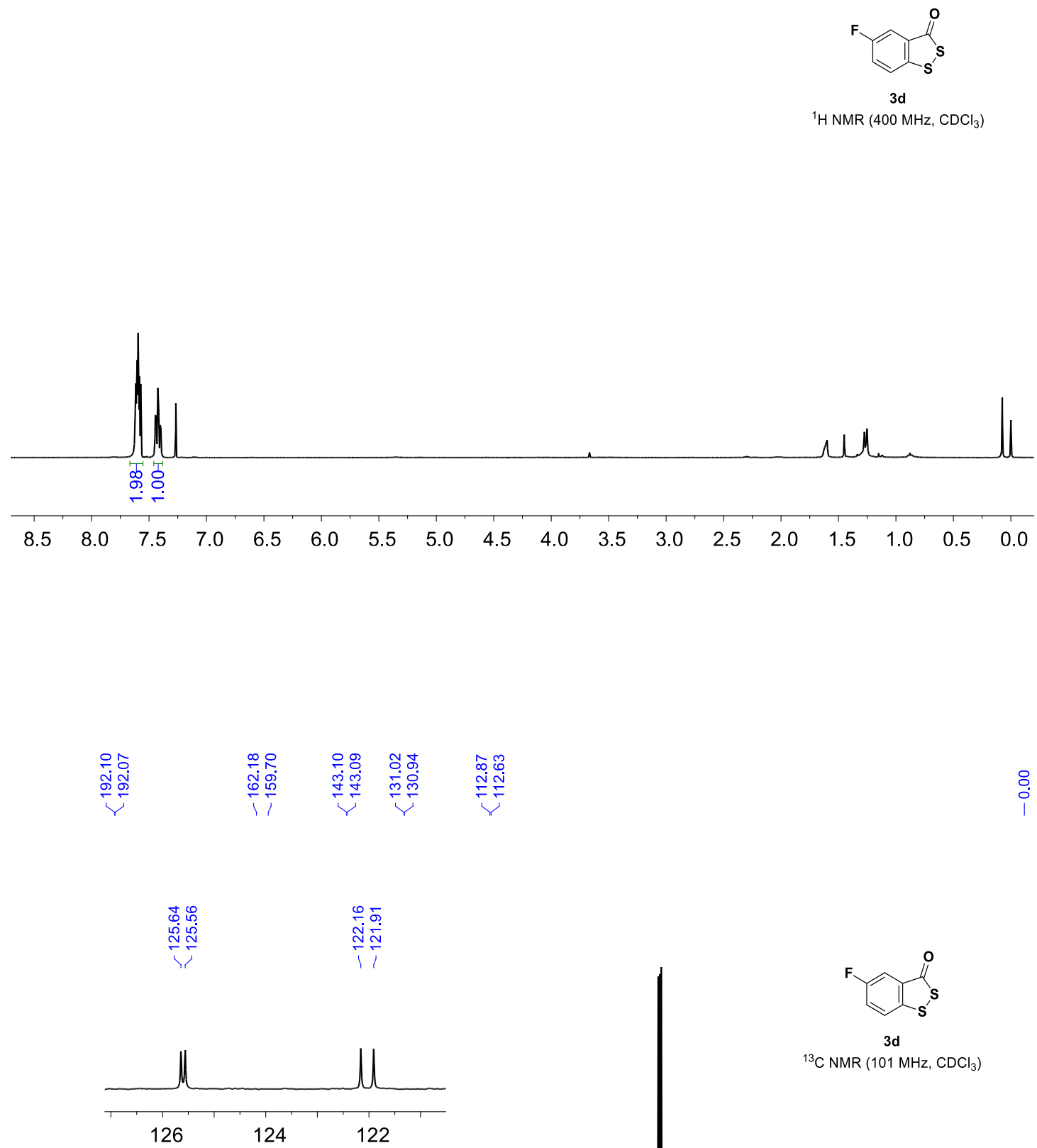

${ }^{13} \mathrm{C}$ NMR $\left(101 \mathrm{MHz}, \mathrm{CDCl}_{3}\right)$

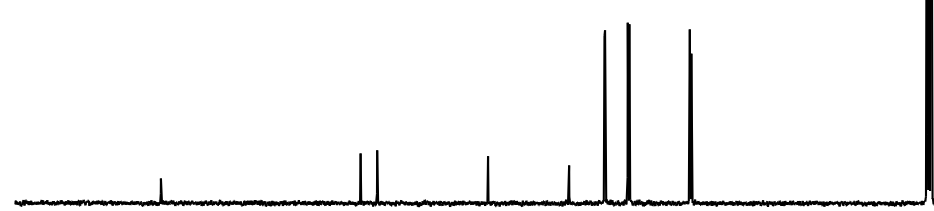




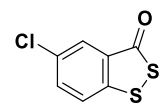

$3 e$

${ }^{1} \mathrm{H} \mathrm{NMR}\left(400 \mathrm{MHz}, \mathrm{CDCl}_{3}\right)$
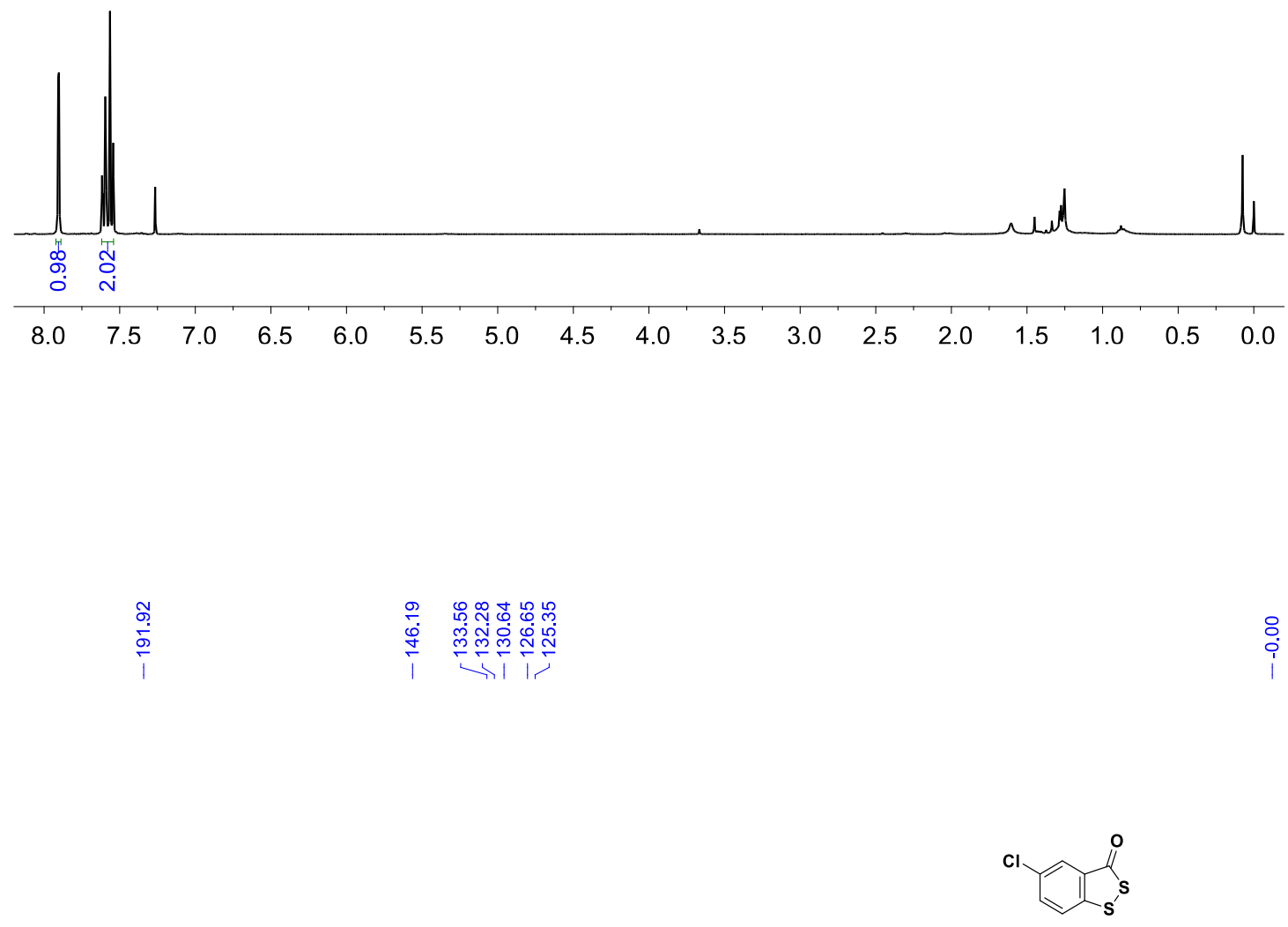

$3 \mathrm{e}$

${ }^{13} \mathrm{C} \mathrm{NMR}\left(101 \mathrm{MHz}, \mathrm{CDCl}_{3}\right)$

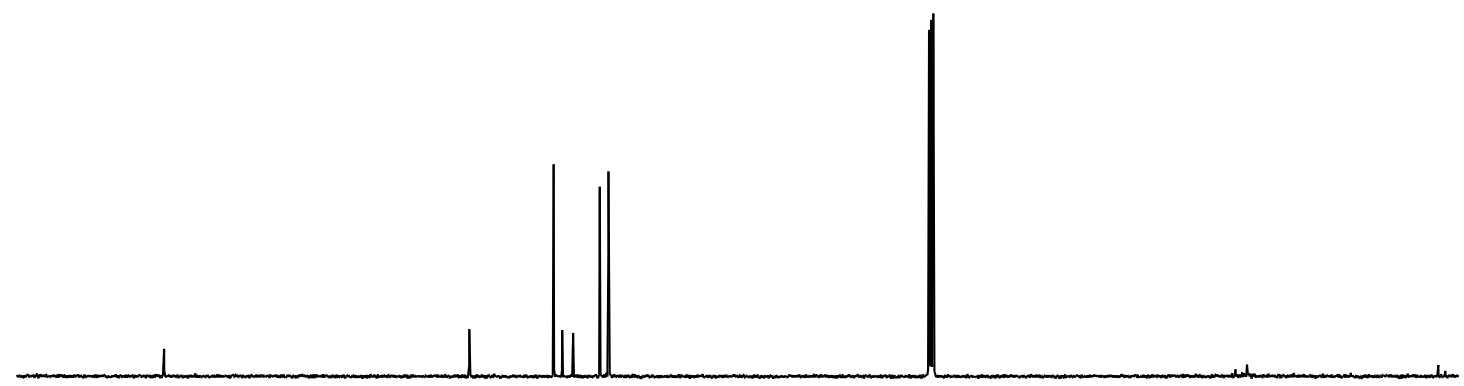

210

$190 \quad 170 \quad 150$

130

110

$\begin{array}{llllllllll}90 & 80 & 70 & 60 & 50 & 40 & 30 & 20 & 10 & 0\end{array}$ 


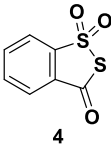

${ }^{1} \mathrm{H} \mathrm{NMR}\left(400 \mathrm{MHz}, \mathrm{CDCl}_{3}\right)$

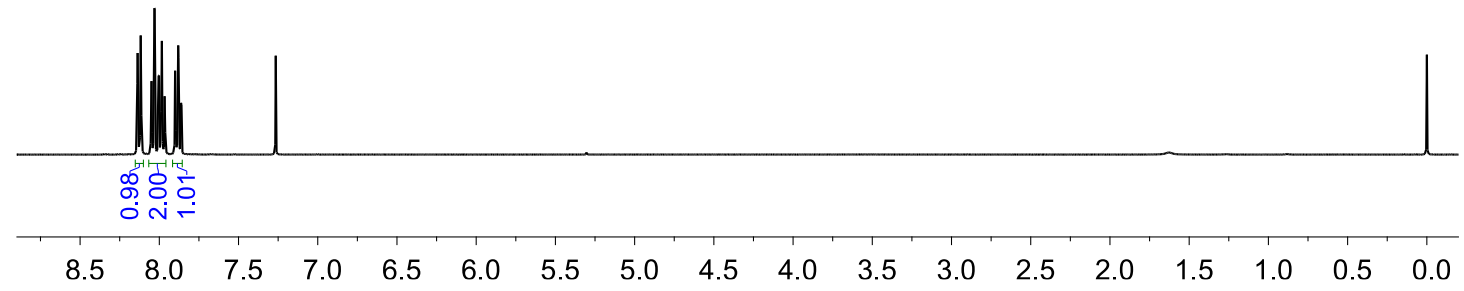

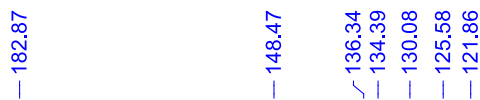

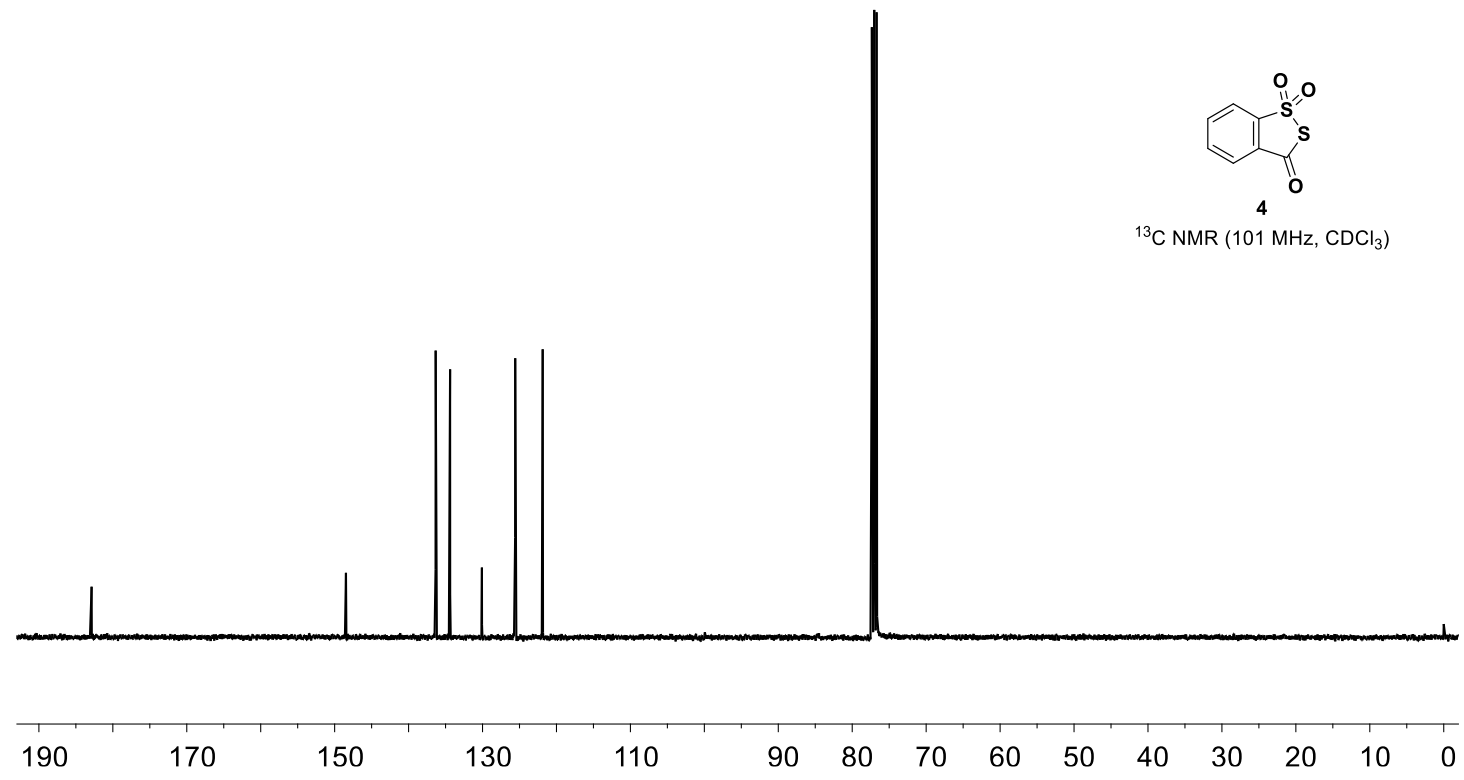




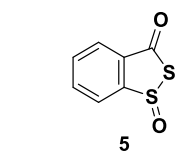

${ }^{1} \mathrm{H}$ NMR $\left(400 \mathrm{MHz}, \mathrm{CDCl}_{3}\right)$

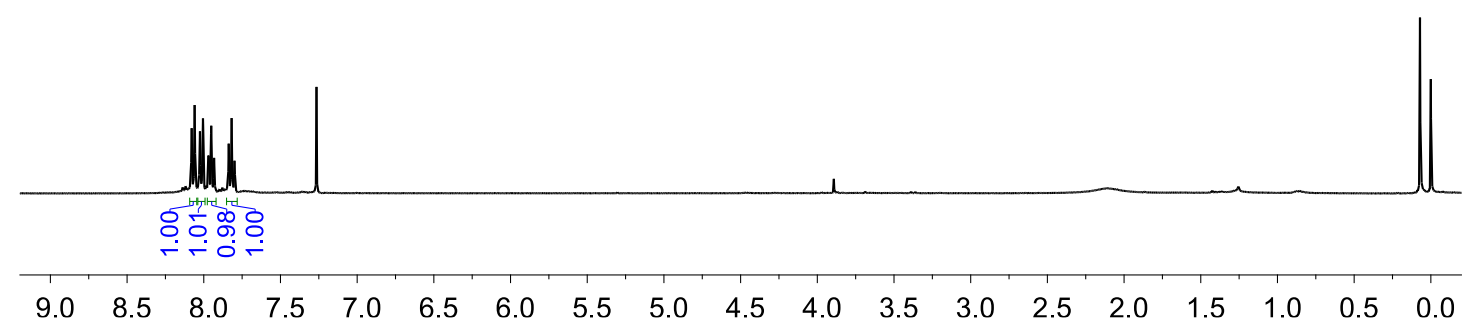

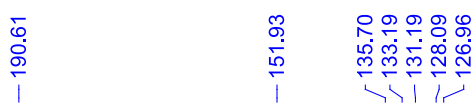
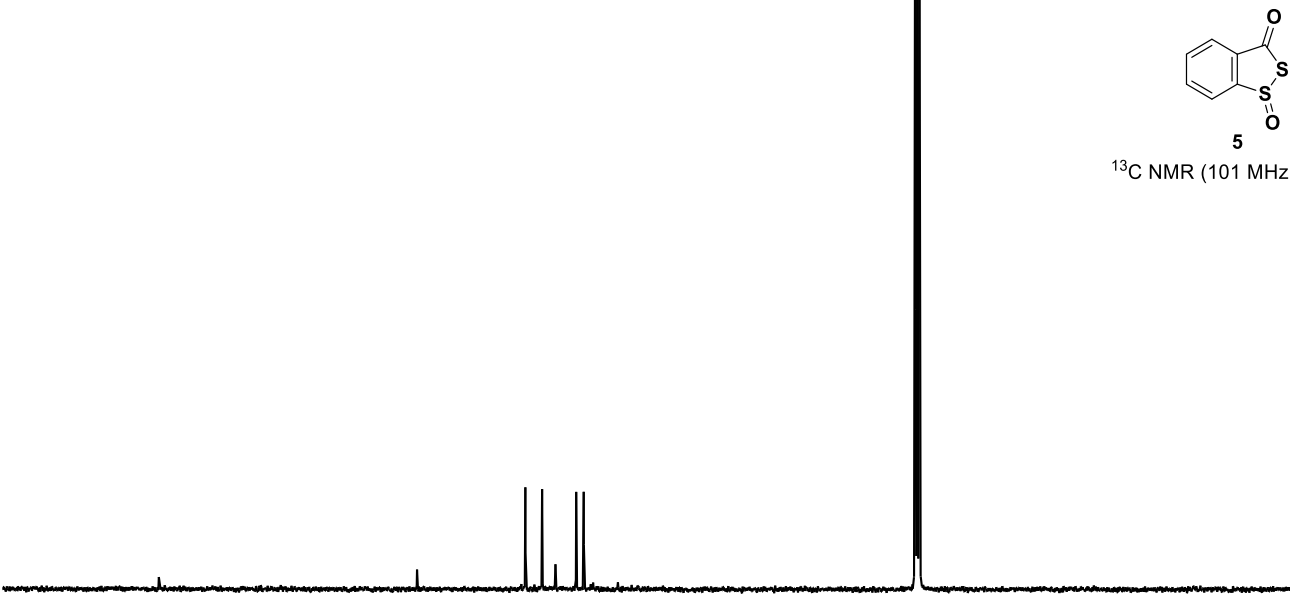

${ }^{13} \mathrm{C}$ NMR $\left(101 \mathrm{MHz}, \mathrm{CDCl}_{3}\right)$

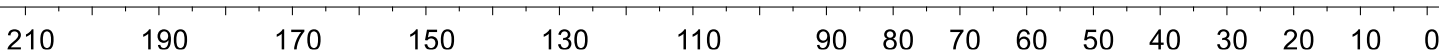




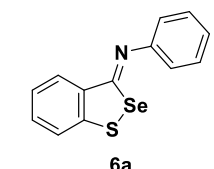

${ }^{1} \mathrm{H}$ NMR $\left(400 \mathrm{MHz}, \mathrm{CDCl}_{3}\right)$
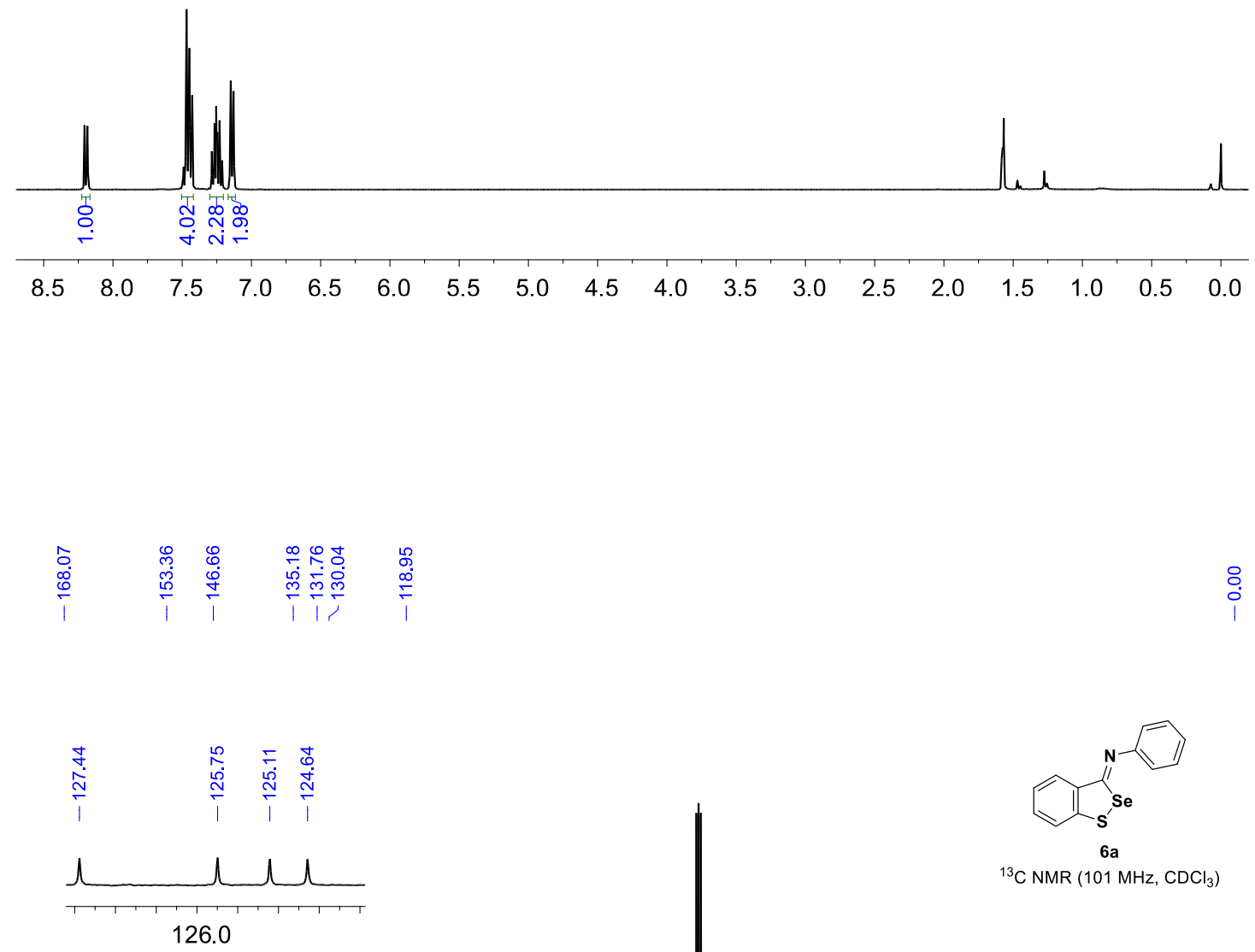

${ }^{13} \mathrm{C}$ NMR $\left(101 \mathrm{MHz}, \mathrm{CDCl}_{3}\right)$

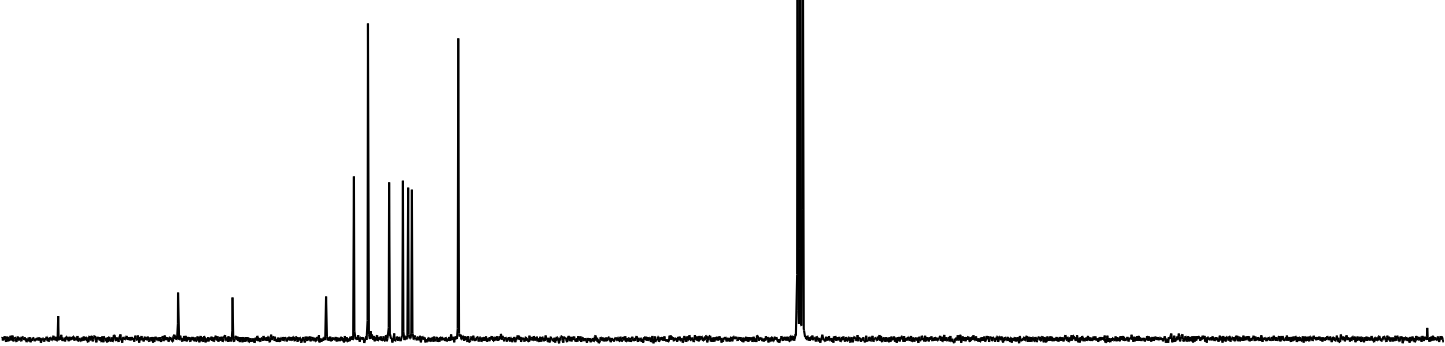

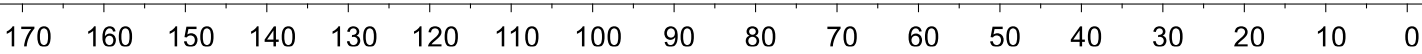




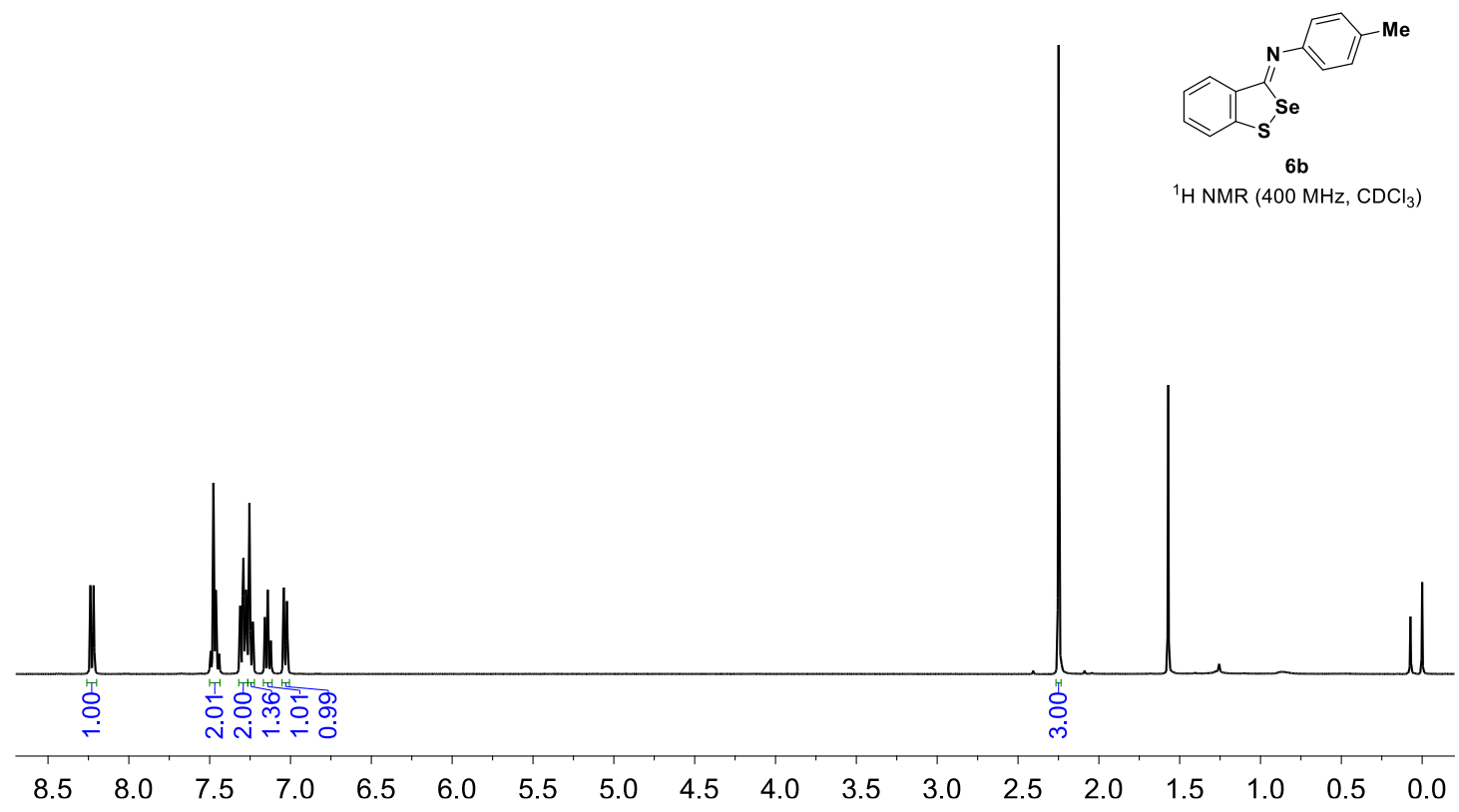

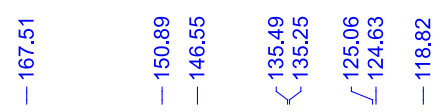
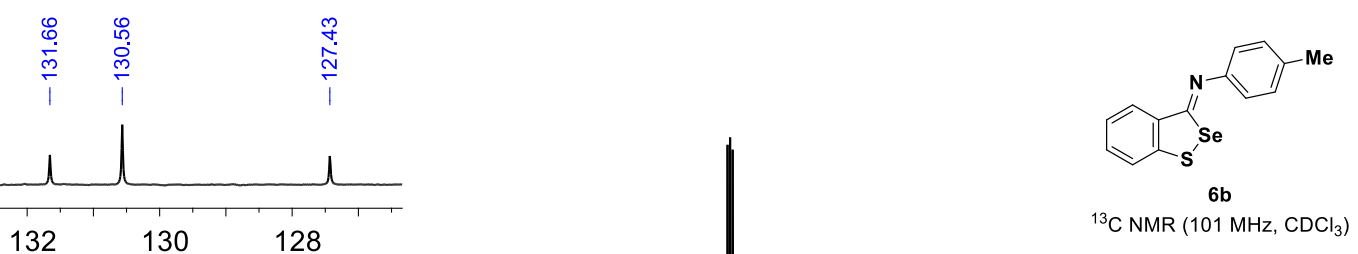

${ }^{13} \mathrm{C}$ NMR $\left(101 \mathrm{MHz}, \mathrm{CDCl}_{3}\right)$

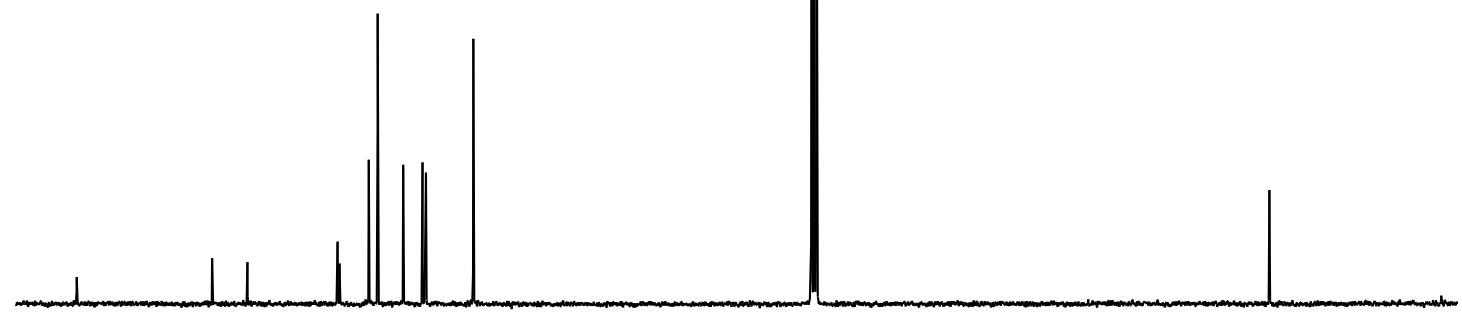

$\begin{array}{llllllllllllllllll}170 & 160 & 150 & 140 & 130 & 120 & 110 & 100 & 90 & 80 & 70 & 60 & 50 & 40 & 30 & 20 & 10 & 0\end{array}$ 


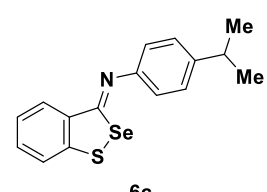

$6 c$

${ }^{1} \mathrm{H} \mathrm{NMR}\left(400 \mathrm{MHz}, \mathrm{CDCl}_{3}\right)$

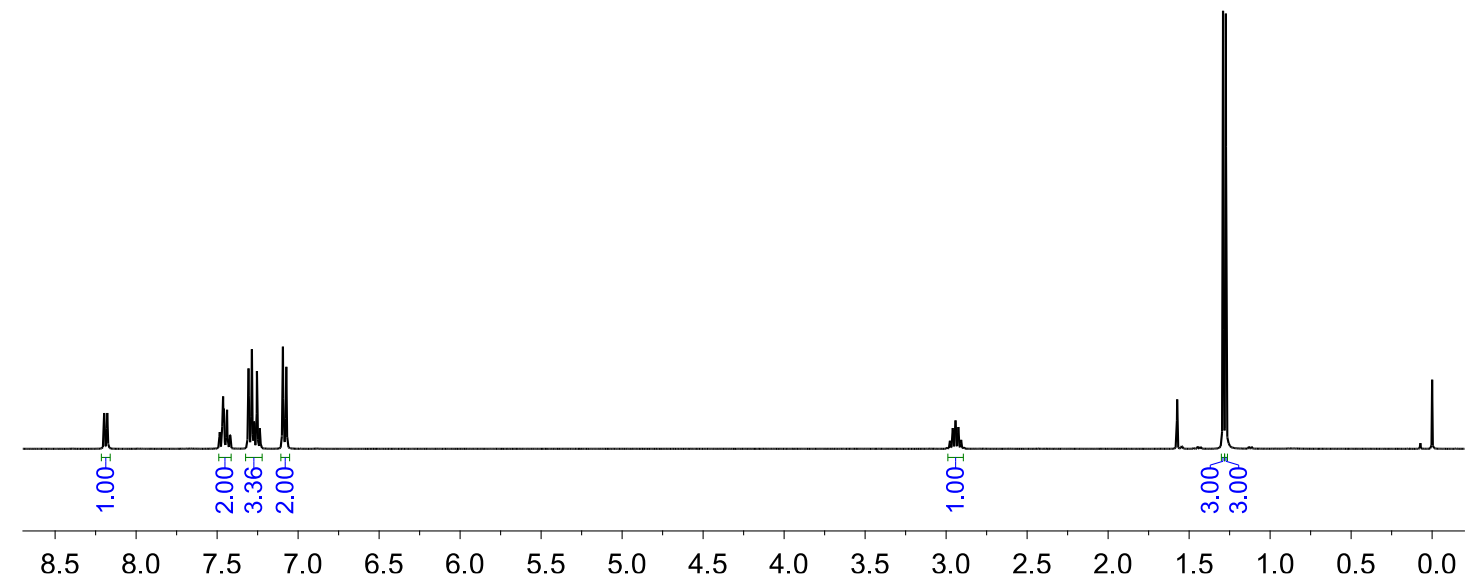

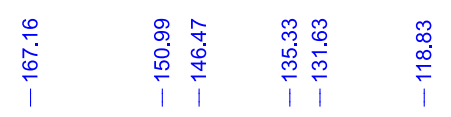

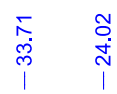

웅
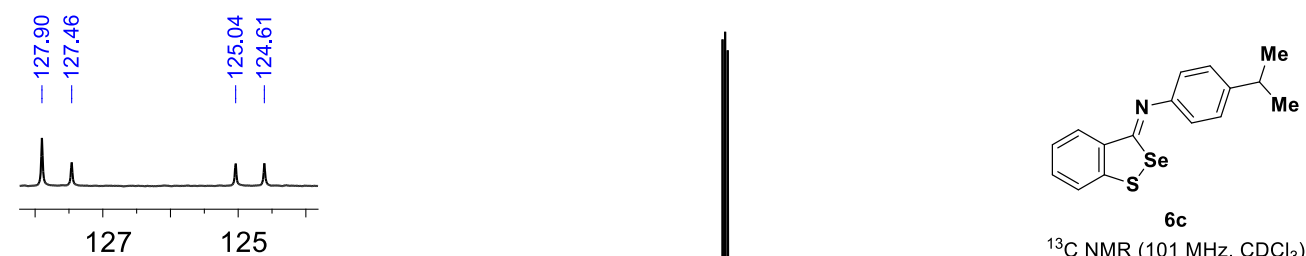

${ }^{13} \mathrm{C} \mathrm{NMR}\left(101 \mathrm{MHz}, \mathrm{CDCl}_{3}\right)$

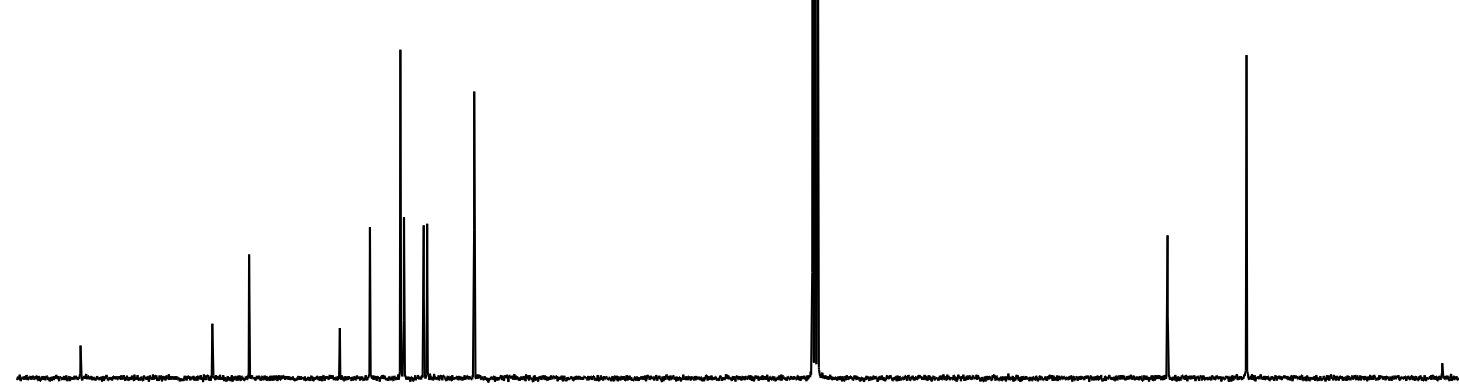

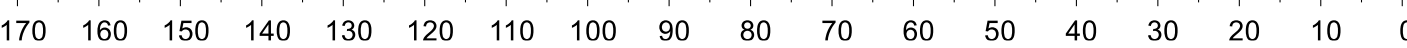




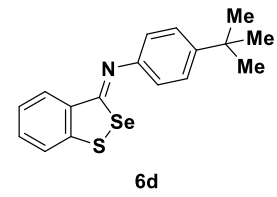

${ }^{1} \mathrm{H}$ NMR $\left(400 \mathrm{MHz}, \mathrm{CDCl}_{3}\right)$

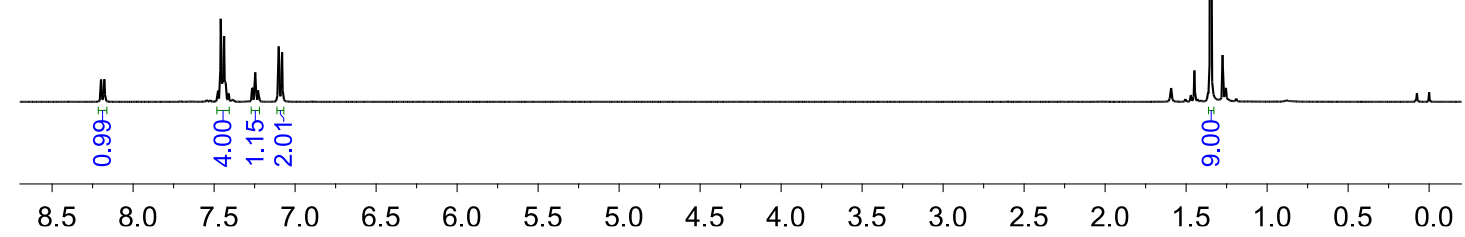

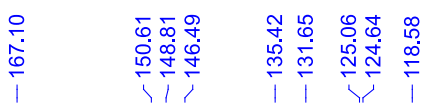

लिए
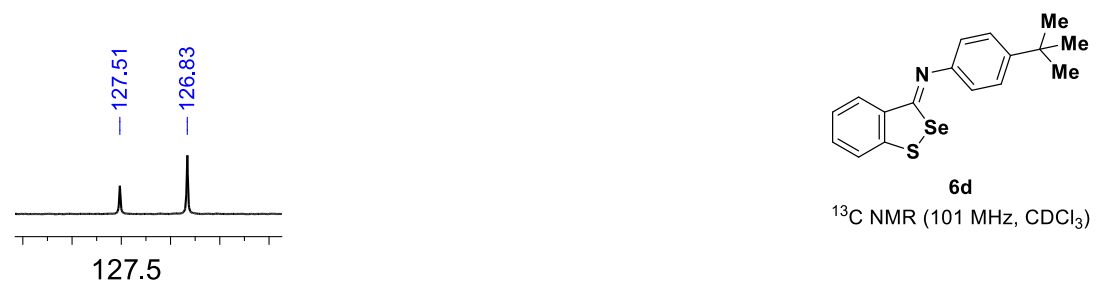

${ }^{13} \mathrm{C} \mathrm{NMR}\left(101 \mathrm{MHz}, \mathrm{CDCl}_{3}\right)$

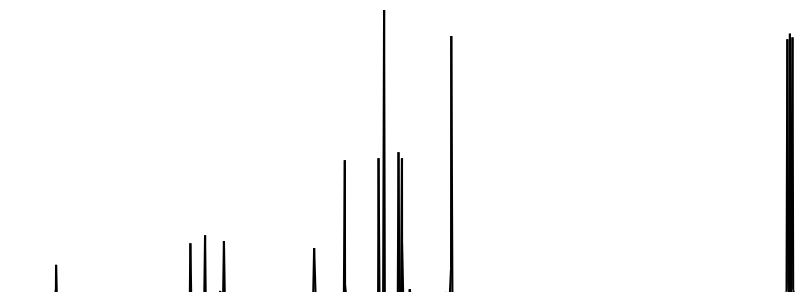

$\begin{array}{lllllllll}170 & 160 & 150 & 140 & 130 & 120 & 110 & 100\end{array}$

$\begin{array}{lllllllll}80 & 70 & 60 & 50 & 40 & 30 & 20 & 10 & 0\end{array}$ 


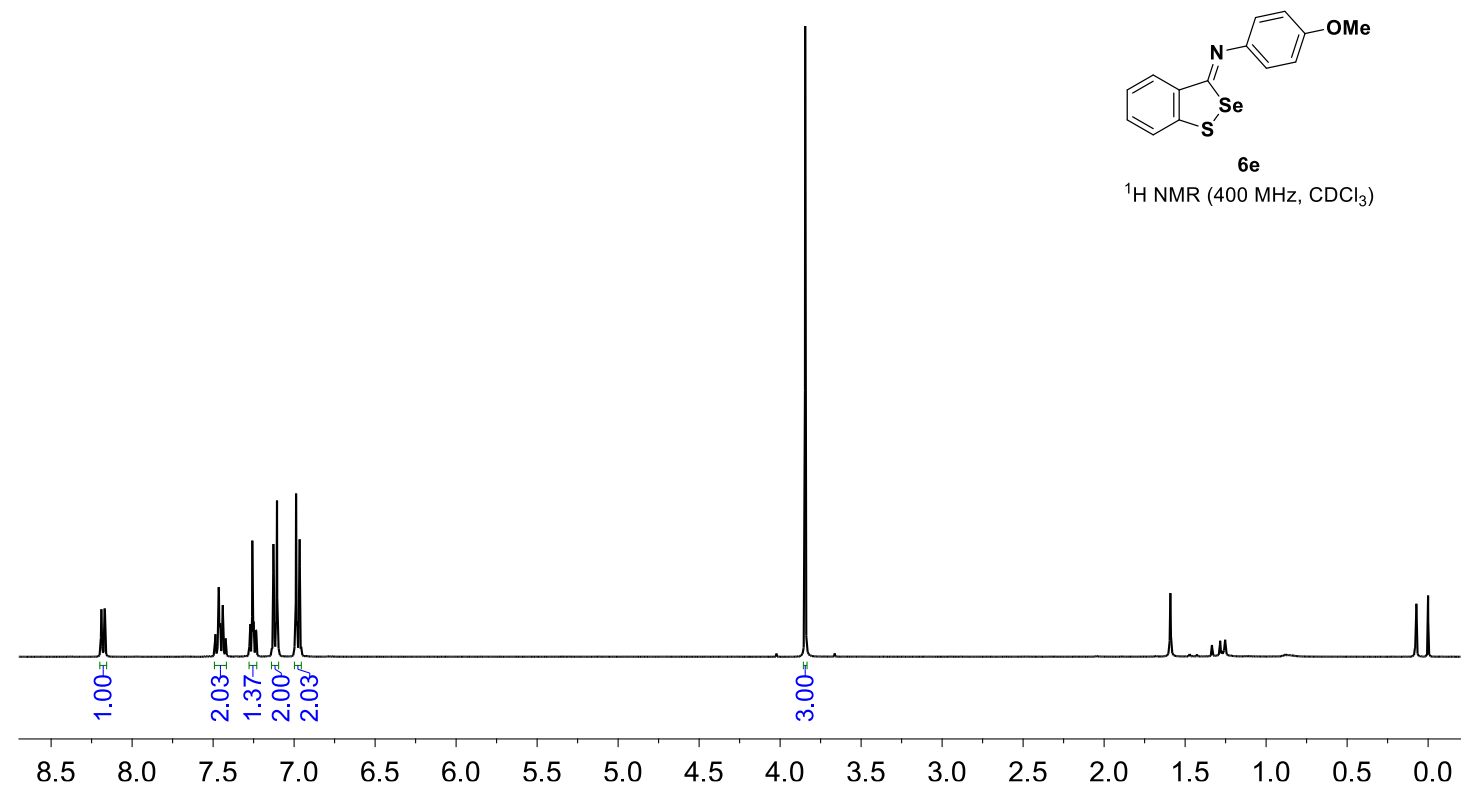

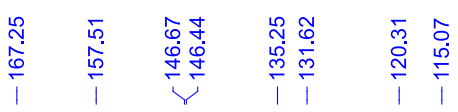

$\underset{\substack{\infty \\ 10}}{10}$
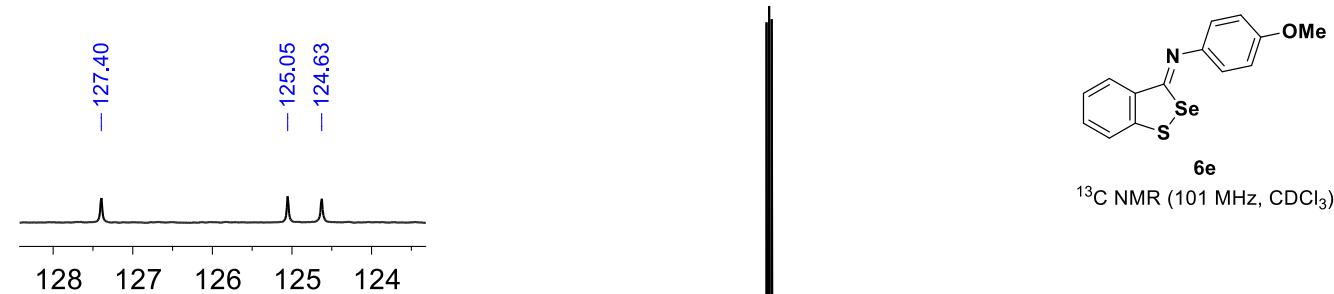

${ }^{13} \mathrm{C} \mathrm{NMR}\left(101 \mathrm{MHz}, \mathrm{CDCl}_{3}\right)$

$\begin{array}{lllll}128 & 127 & 126 & 125 & 124\end{array}$

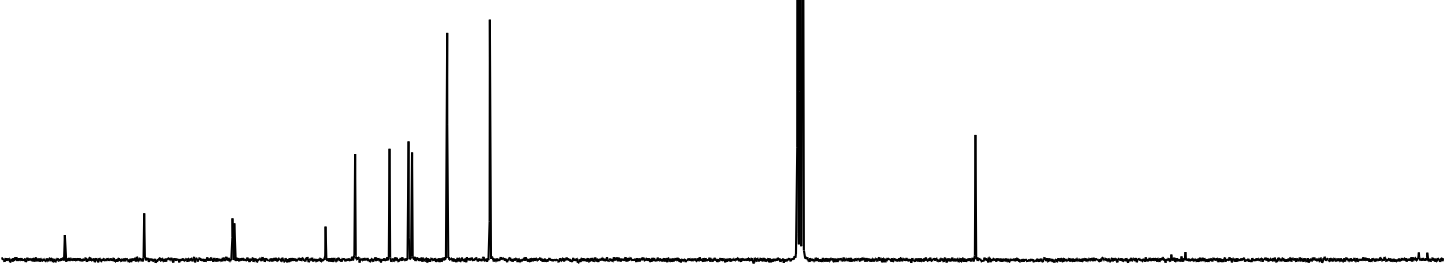

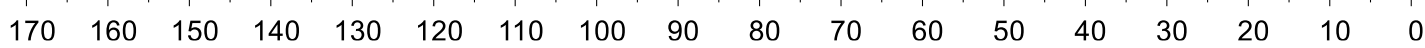




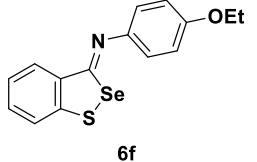

${ }^{1} \mathrm{H}$ NMR $\left(400 \mathrm{MHz}, \mathrm{CDCl}_{3}\right)$

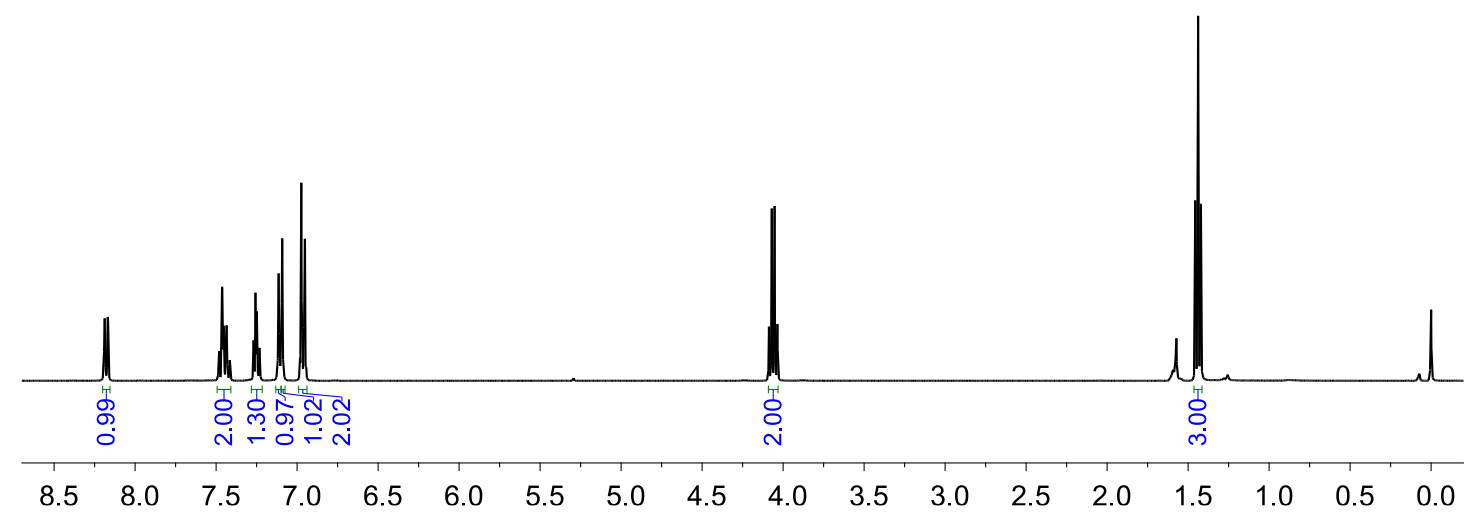

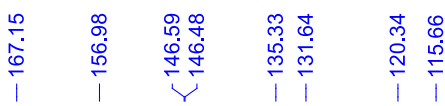
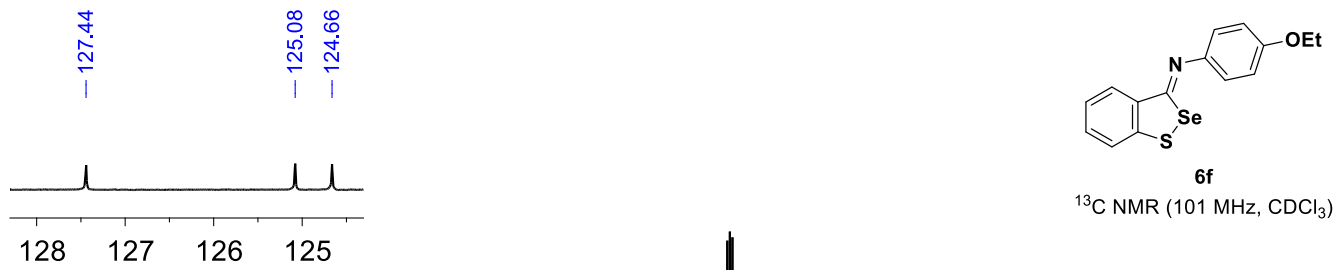

${ }^{13} \mathrm{C} \mathrm{NMR}\left(101 \mathrm{MHz}, \mathrm{CDCl}_{3}\right)$

$\begin{array}{llll}128 & 127 & 126 & 125\end{array}$

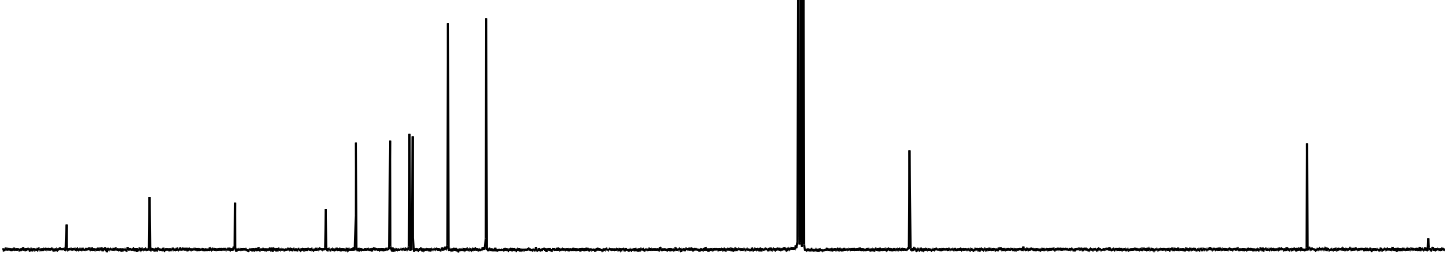

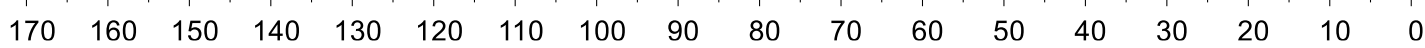




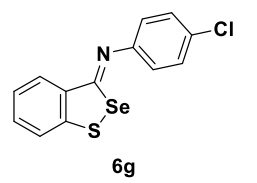

${ }^{1} \mathrm{H}$ NMR $\left(400 \mathrm{MHz}, \mathrm{CDCl}_{3}\right)$

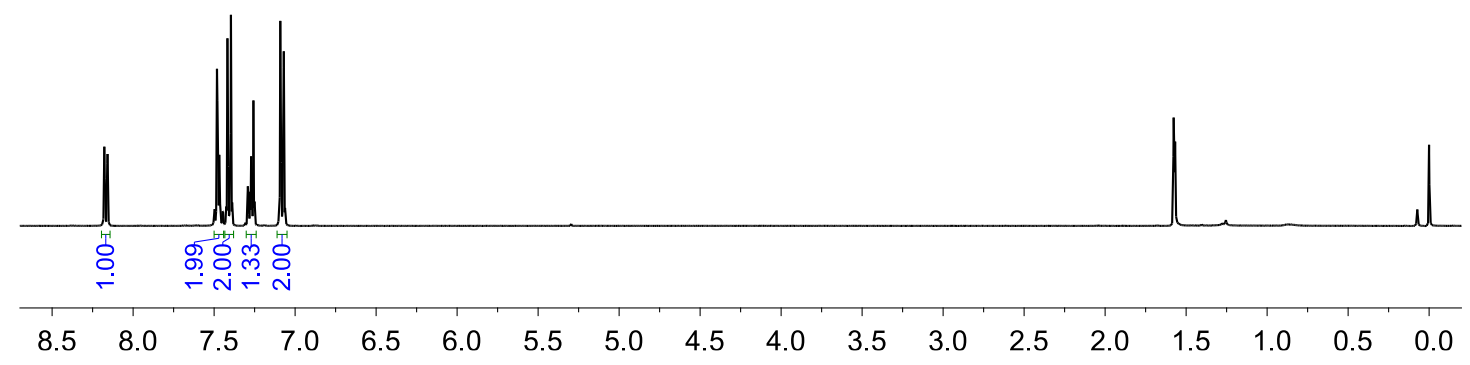

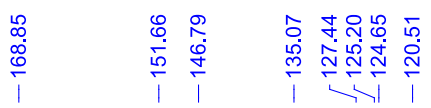
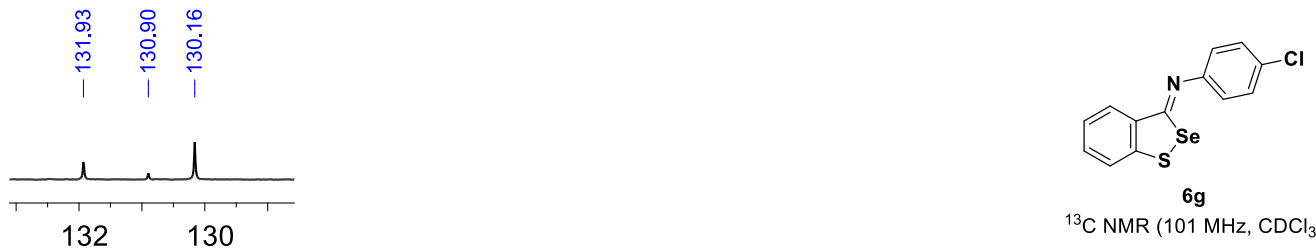

${ }^{13} \mathrm{C} \mathrm{NMR}\left(101 \mathrm{MHz}, \mathrm{CDCl}_{3}\right)$

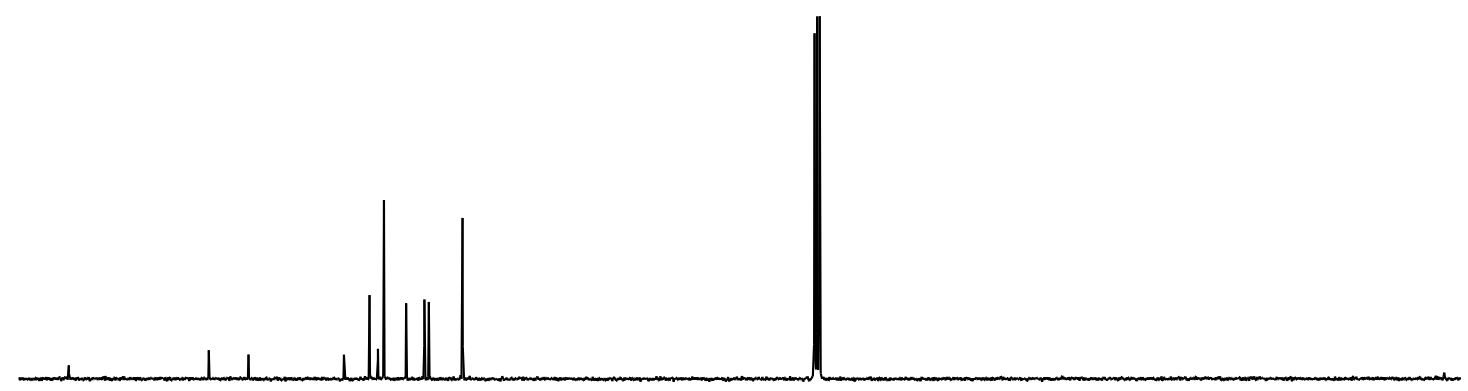

$\begin{array}{llllllllllllllllll}170 & 160 & 150 & 140 & 130 & 120 & 110 & 100 & 90 & 80 & 70 & 60 & 50 & 40 & 30 & 20 & 10 & 0\end{array}$ 


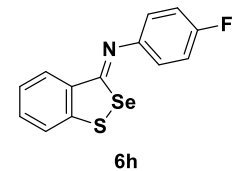

${ }^{1} \mathrm{H} \mathrm{NMR}\left(400 \mathrm{MHz}, \mathrm{CDCl}_{3}\right)$

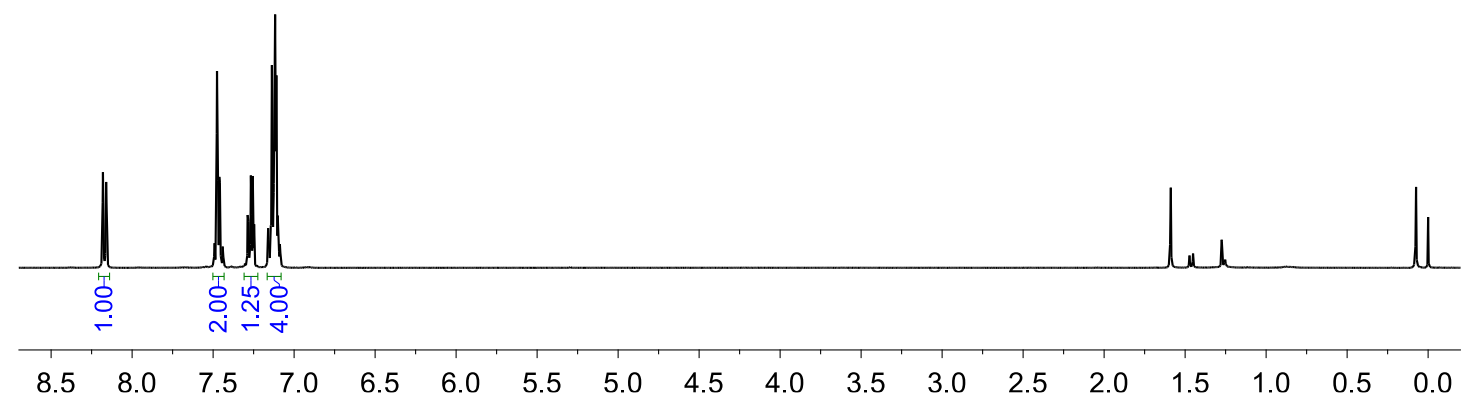

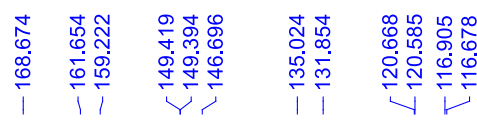
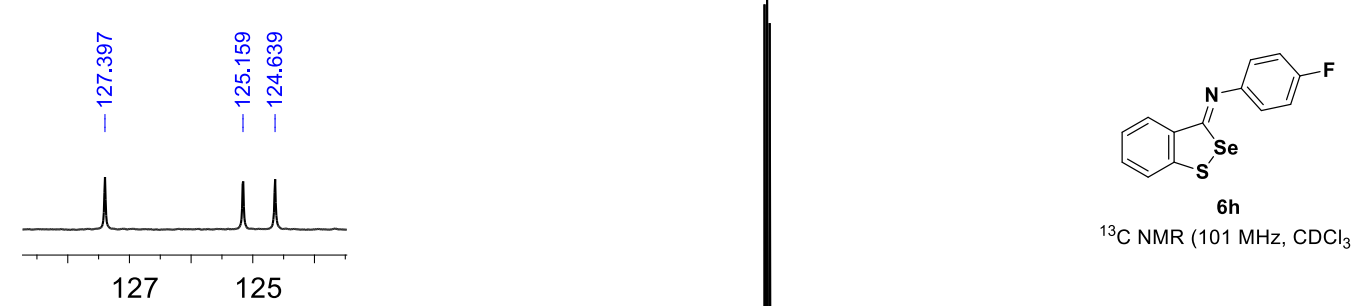

${ }^{13} \mathrm{C} \mathrm{NMR}\left(101 \mathrm{MHz}, \mathrm{CDCl}_{3}\right)$

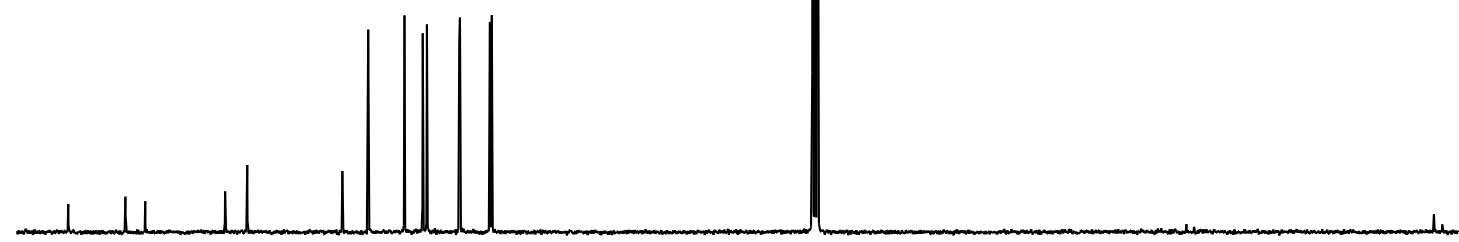

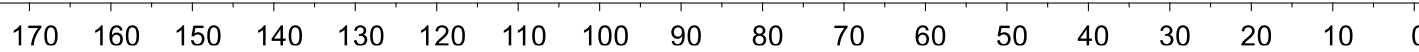



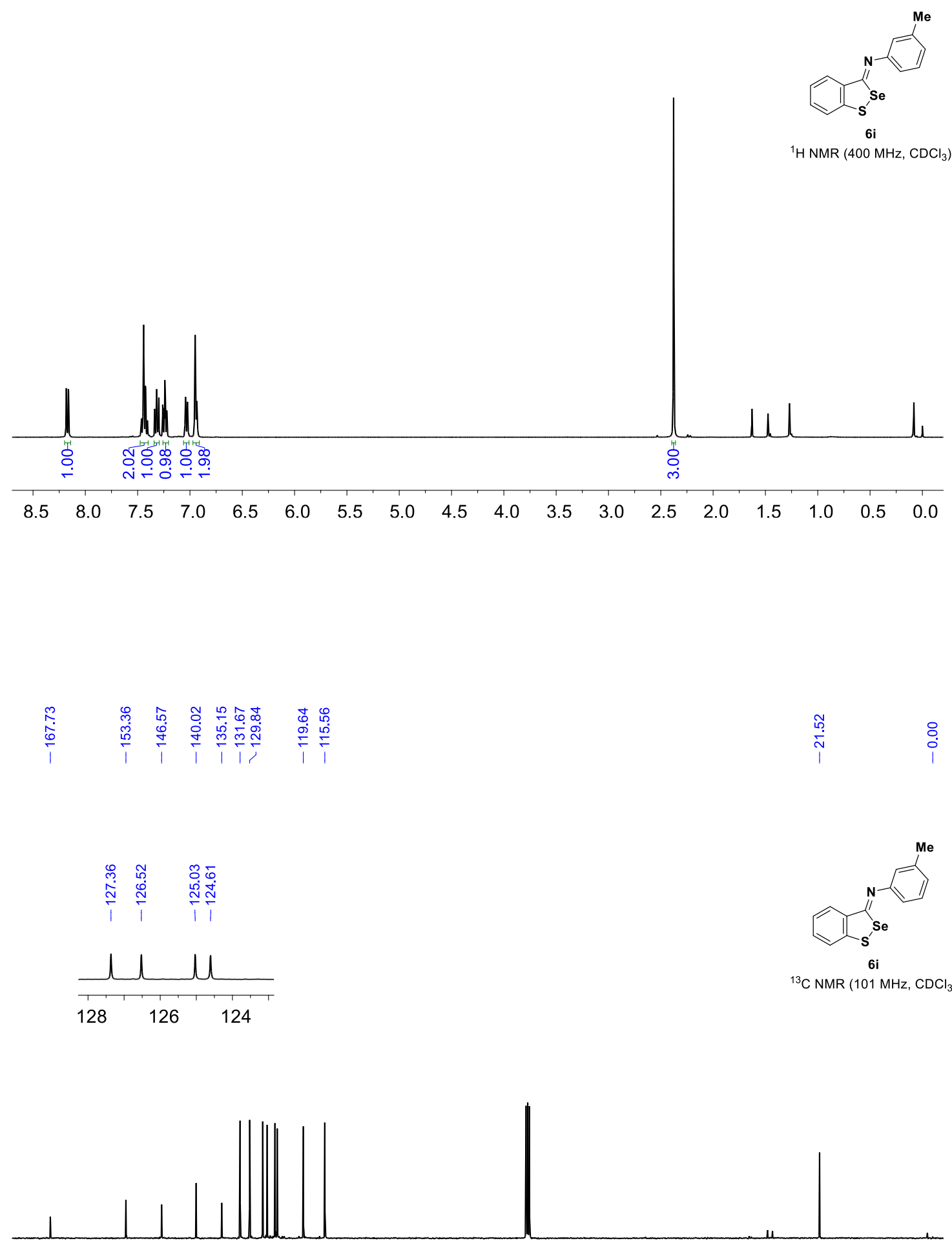

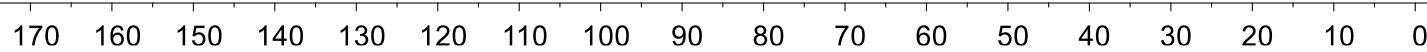




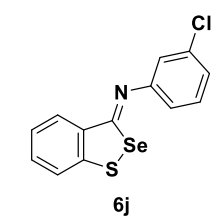

${ }^{1} \mathrm{H}$ NMR $\left(400 \mathrm{MHz}, \mathrm{CDCl}_{3}\right)$

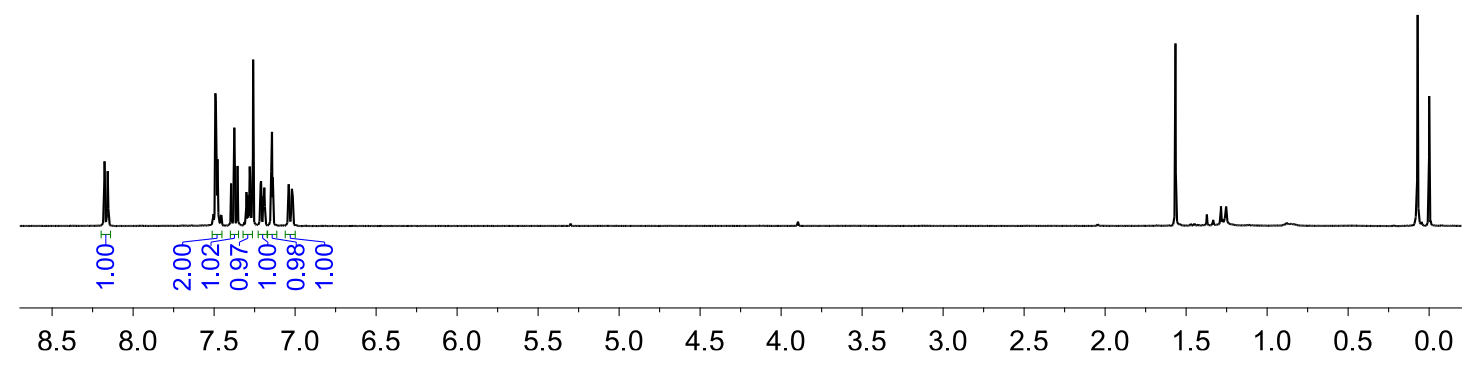

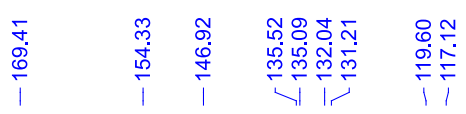
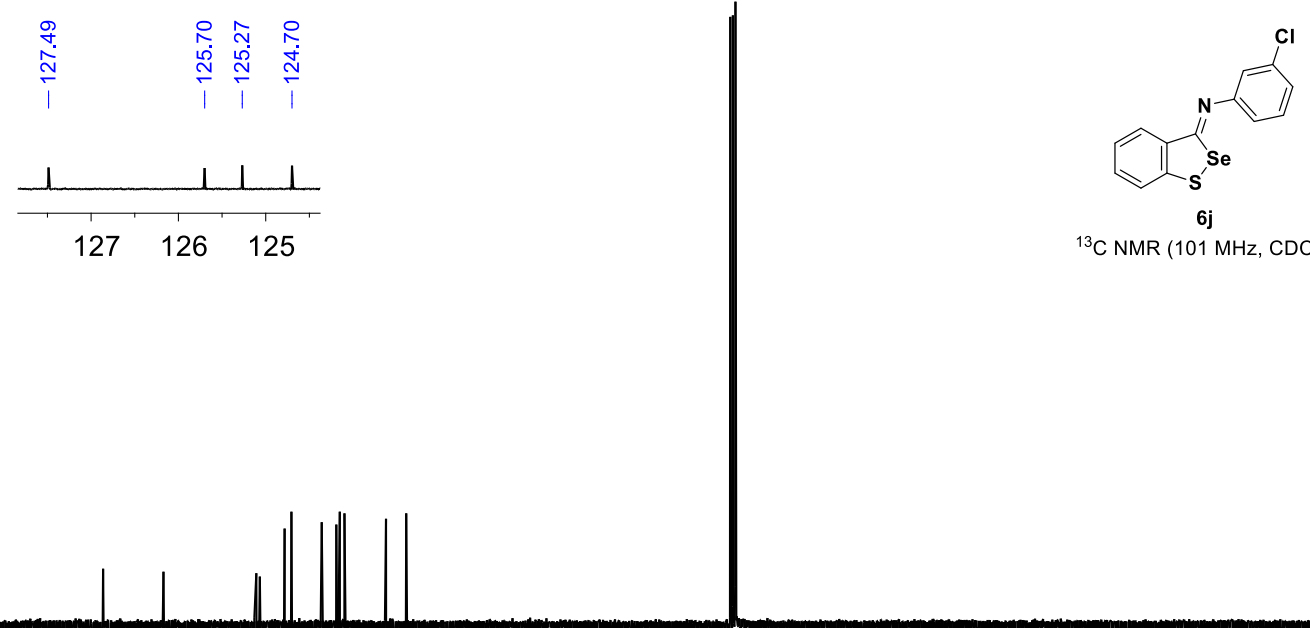

${ }^{13} \mathrm{C}$ NMR (101 MHz, $\left.\mathrm{CDCl}_{3}\right)$

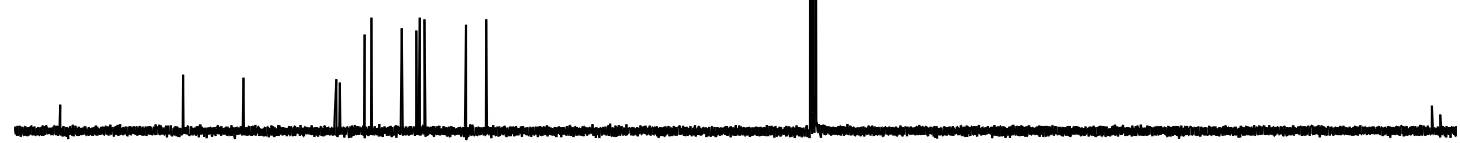

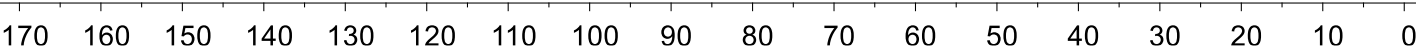




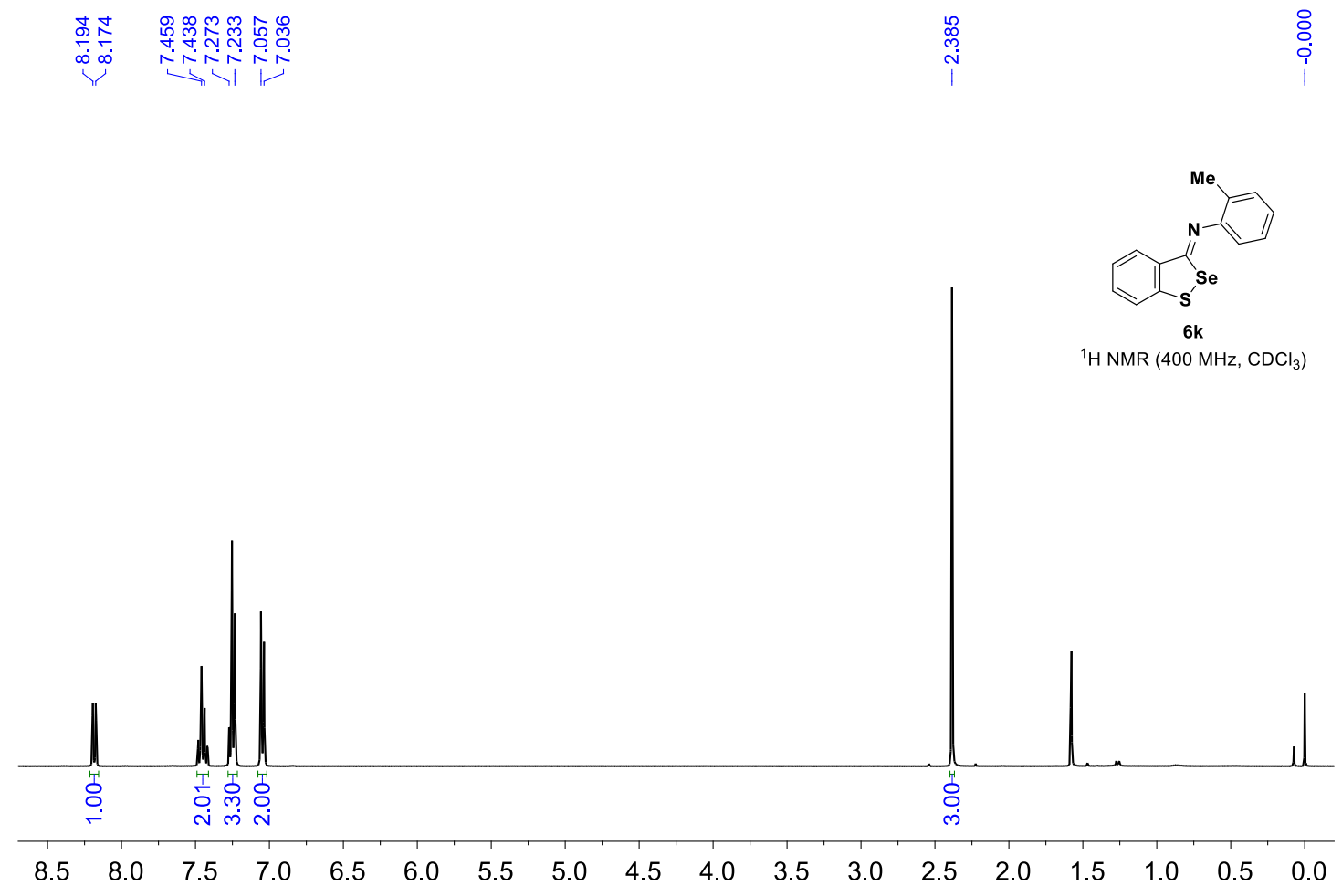

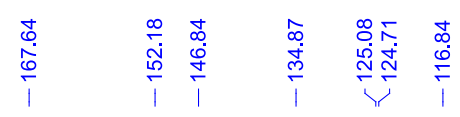

$\stackrel{\substack{10 \\ 1}}{\circ} \quad$
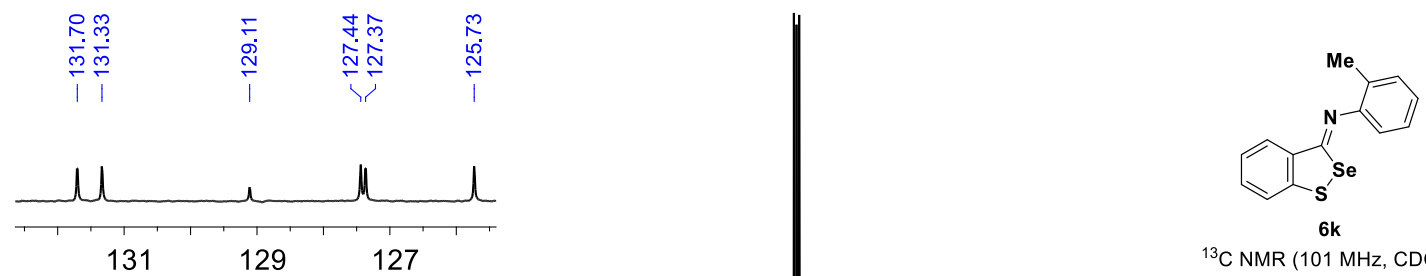

${ }^{13} \mathrm{C}$ NMR $\left(101 \mathrm{MHz}, \mathrm{CDCl}_{3}\right)$

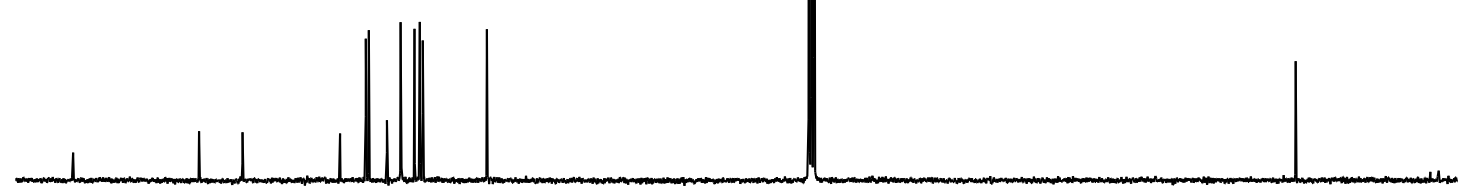

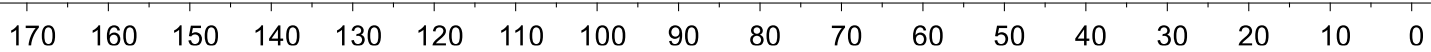




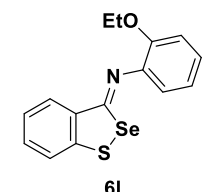

${ }^{1} \mathrm{H} \mathrm{NMR}\left(400 \mathrm{MHz}, \mathrm{CDCl}_{3}\right)$
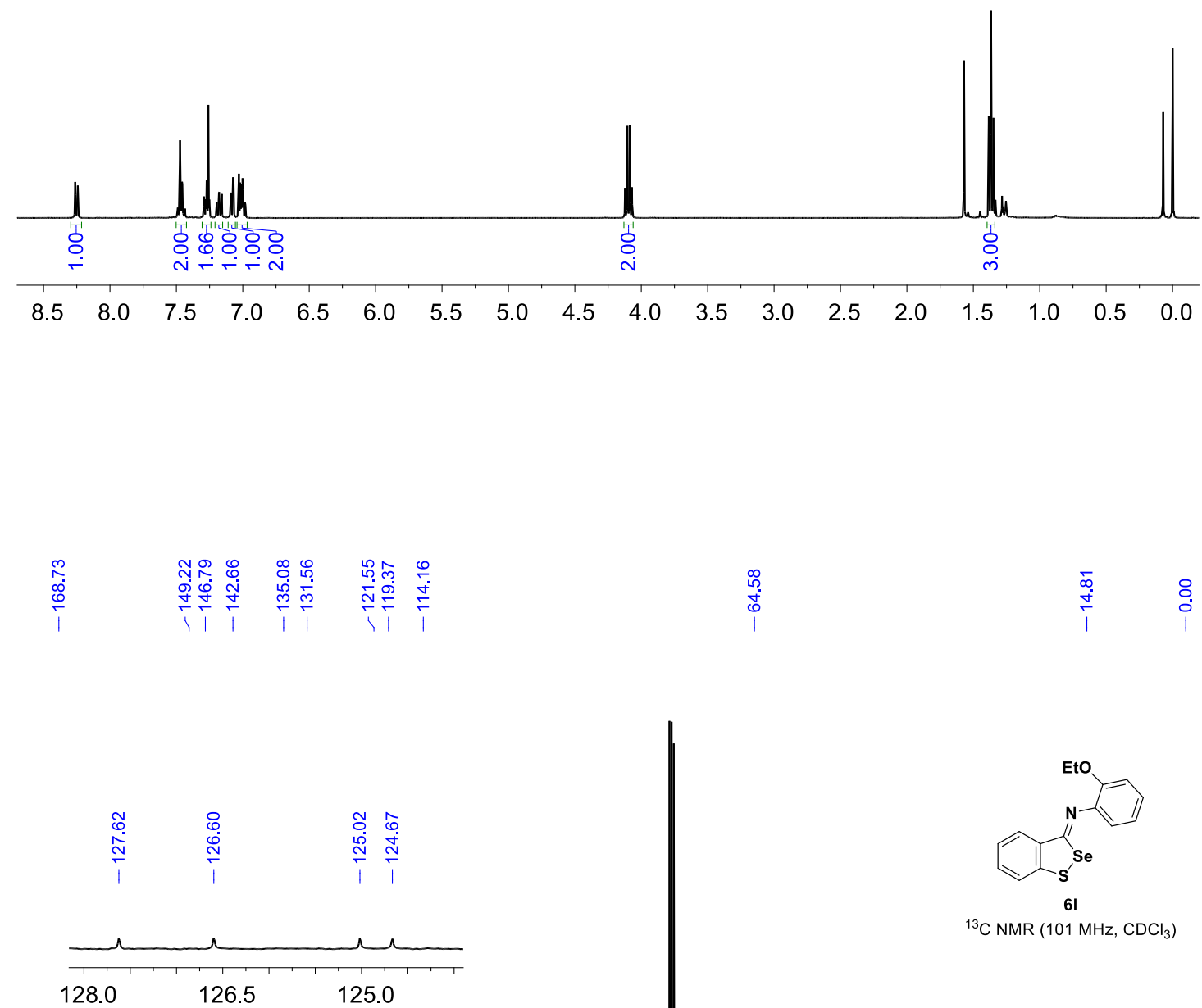

128

126.5

${ }^{13} \mathrm{C}$ NMR $\left(101 \mathrm{MHz}, \mathrm{CDCl}_{3}\right)$

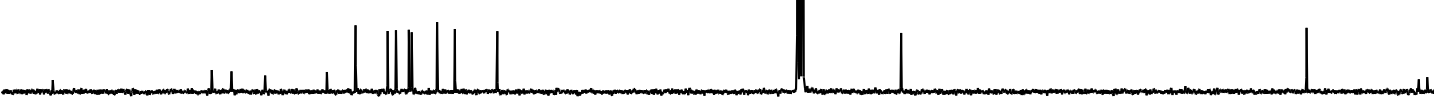

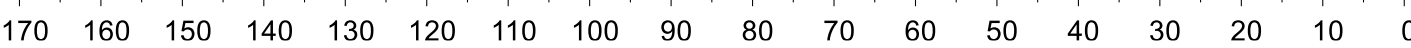




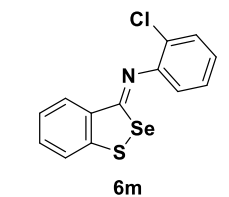

${ }^{1} \mathrm{H} \mathrm{NMR}\left(400 \mathrm{MHz}, \mathrm{CDCl}_{3}\right)$

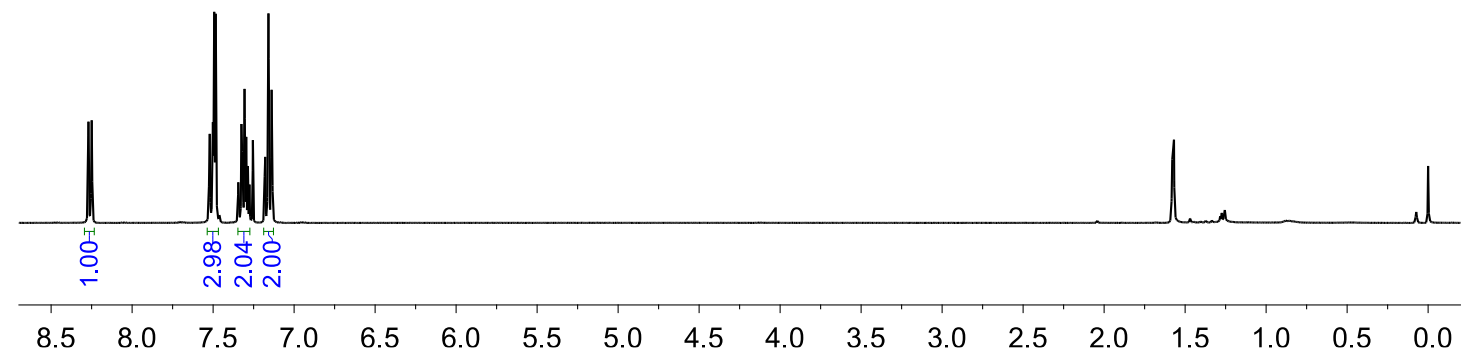

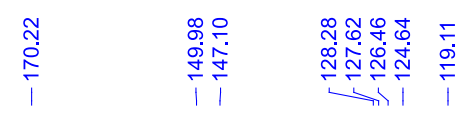
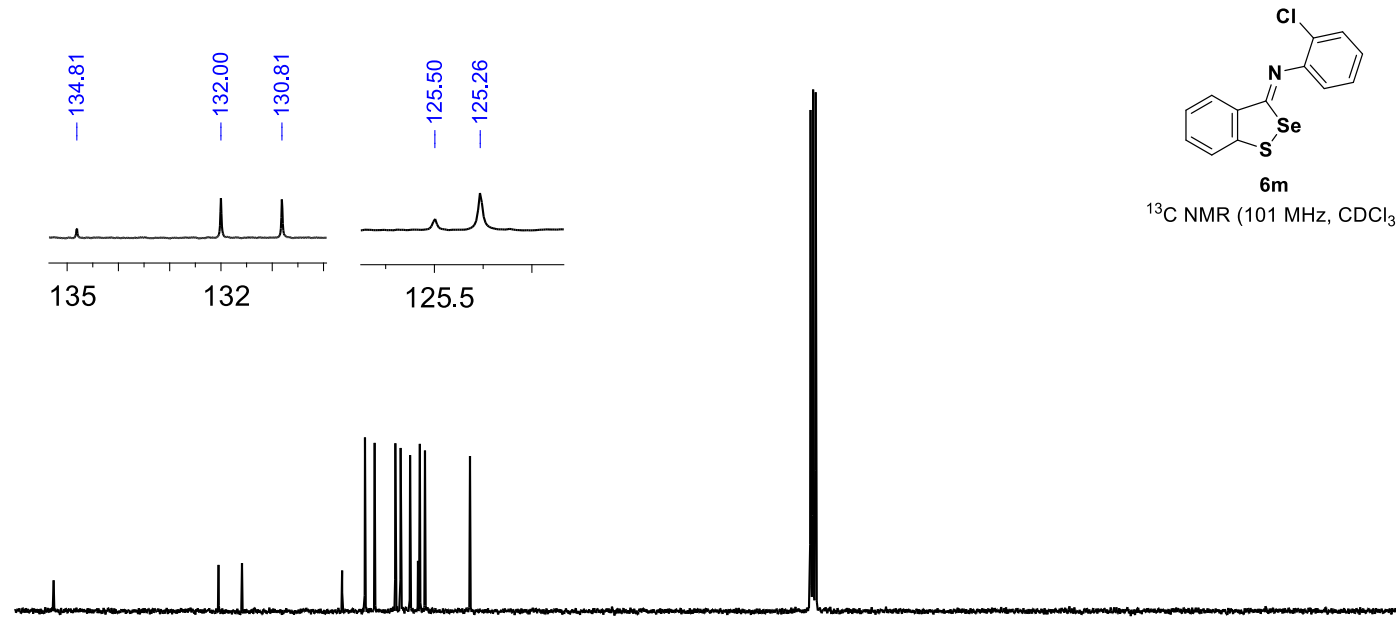

${ }^{13} \mathrm{C}$ NMR $\left(101 \mathrm{MHz}, \mathrm{CDCl}_{3}\right)$

$$
135
$$

$170 \quad 160 \quad 150$

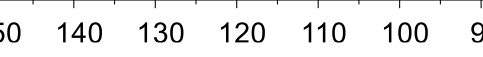

$8070 \quad 60$

$60 \quad 50$

$\begin{array}{lllll}40 & 30 & 20 & 10 & 0\end{array}$ 

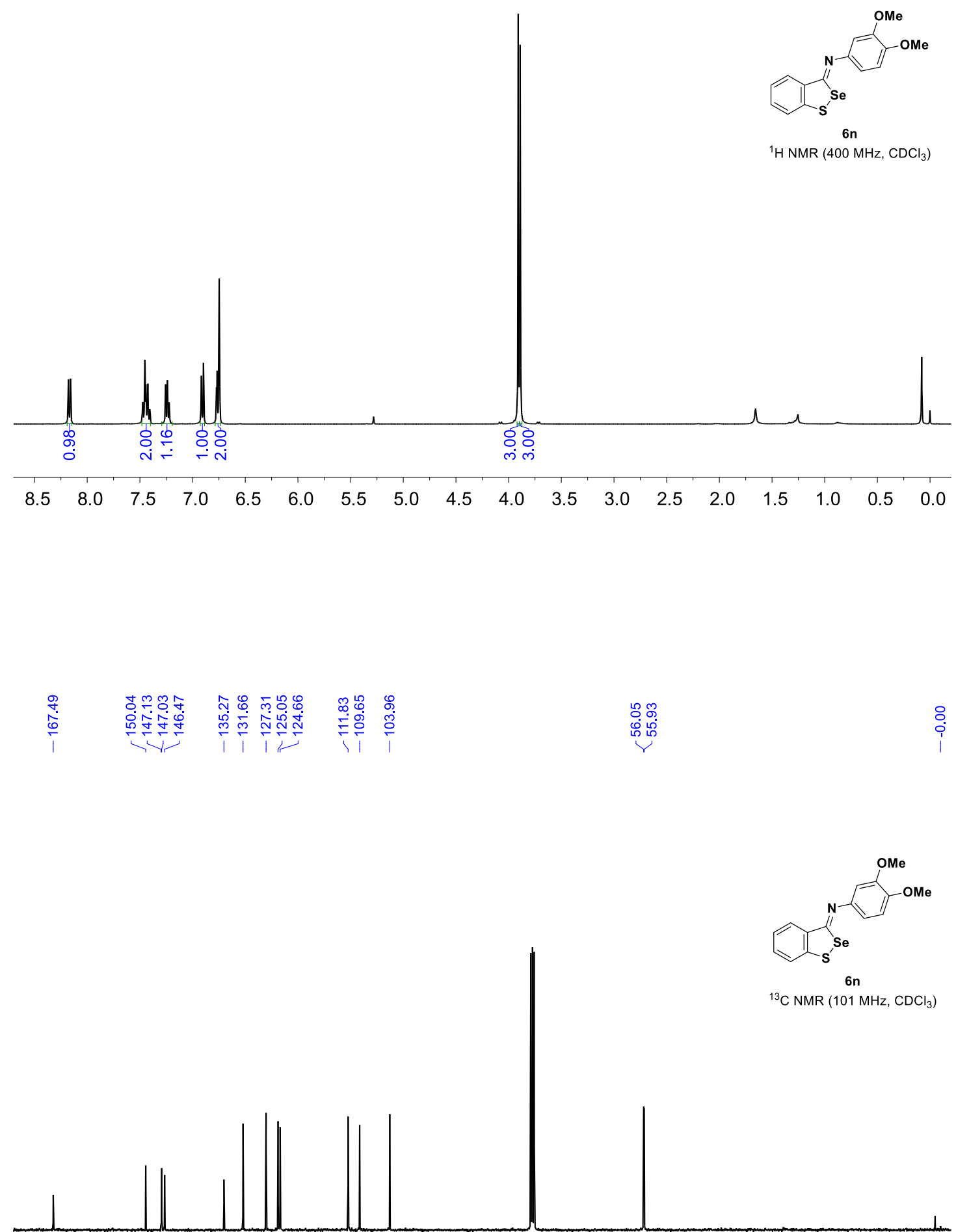

$\left.{ }^{13} \mathrm{C} \mathrm{NMR} \mathrm{(101} \mathrm{MHz,} \mathrm{CDCl}_{3}\right)$

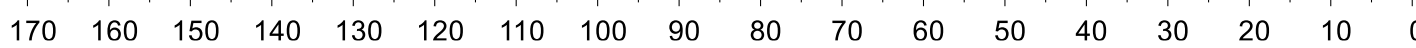




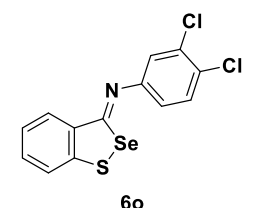

${ }^{1} \mathrm{H} \mathrm{NMR}\left(400 \mathrm{MHz}, \mathrm{CDCl}_{3}\right)$

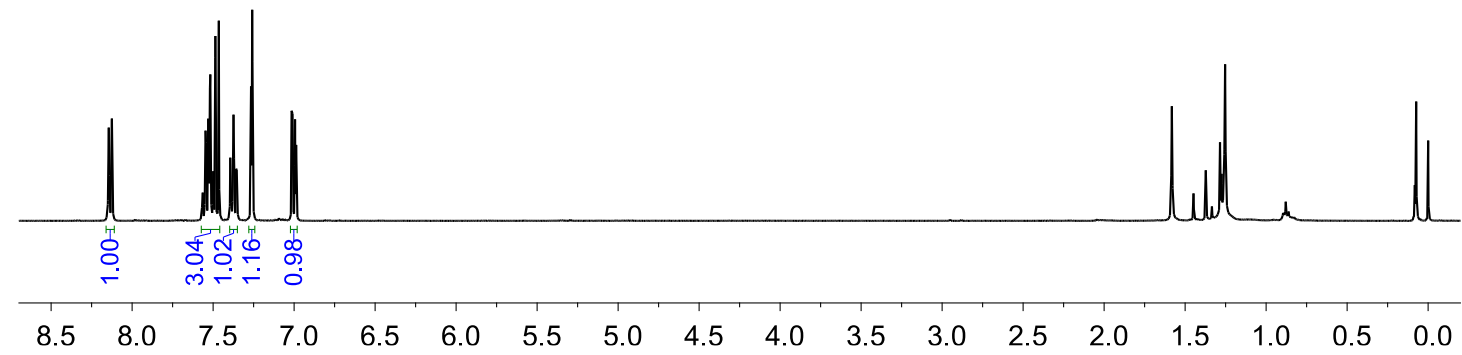

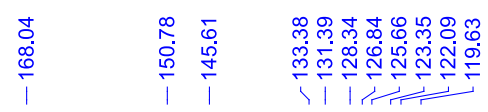
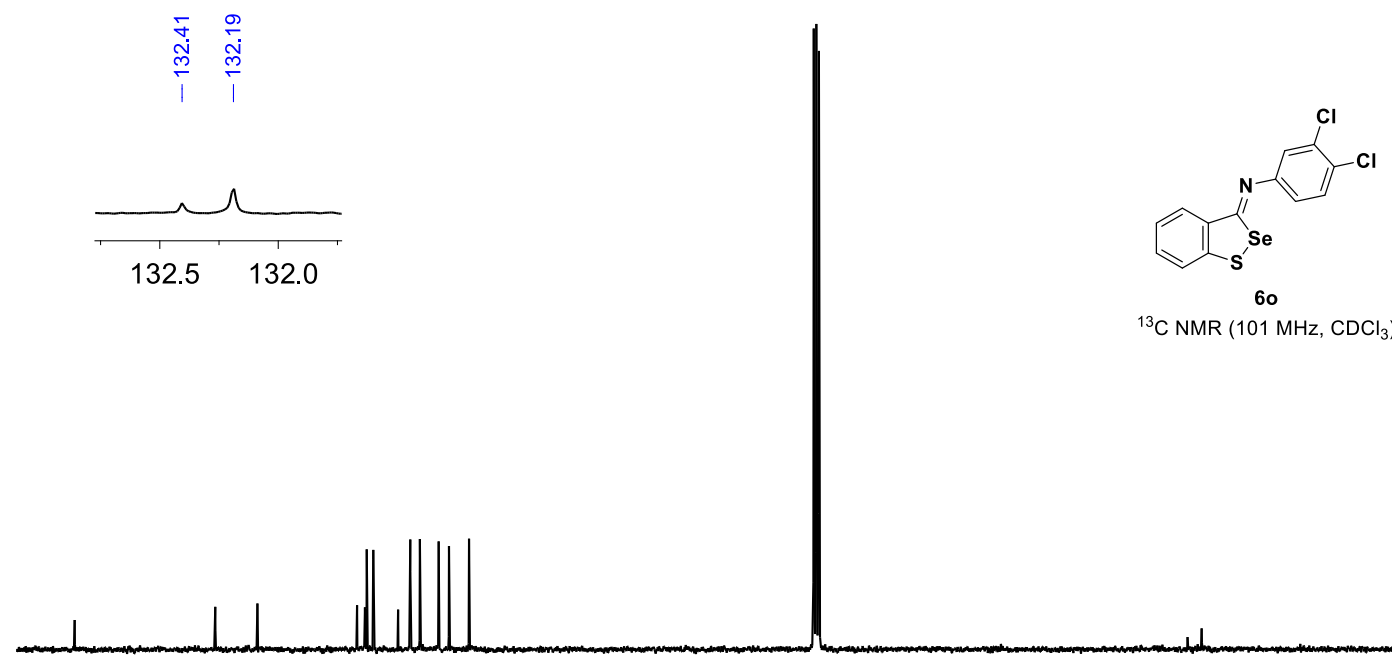

${ }^{13} \mathrm{C} \mathrm{NMR}\left(101 \mathrm{MHz}, \mathrm{CDCl}_{3}\right)$

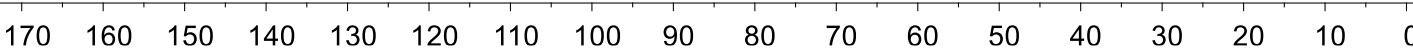




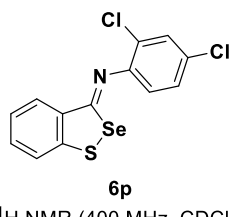

${ }^{1} \mathrm{H} \mathrm{NMR}\left(400 \mathrm{MHz}, \mathrm{CDCl}_{3}\right)$

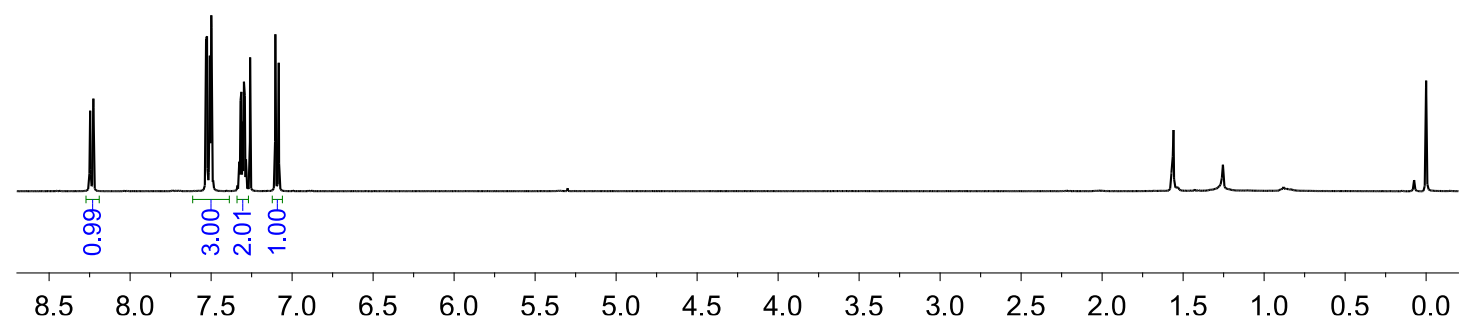

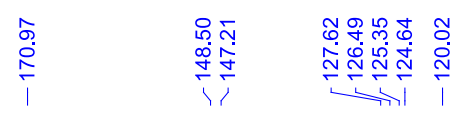
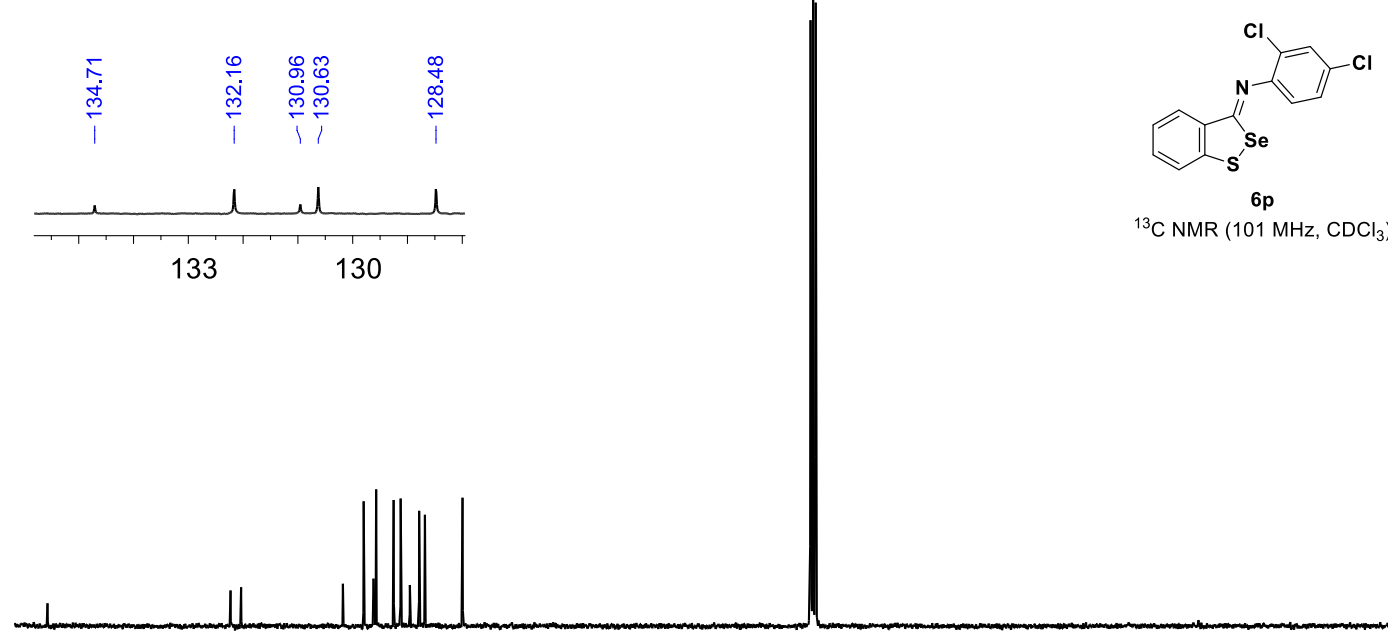

${ }^{13} \mathrm{C} \mathrm{NMR}\left(101 \mathrm{MHz}, \mathrm{CDCl}_{3}\right)$

$\begin{array}{llllllllllllllllll}170 & 160 & 150 & 140 & 130 & 120 & 110 & 100 & 90 & 80 & 70 & 60 & 50 & 40 & 30 & 20 & 10 & 0\end{array}$ 


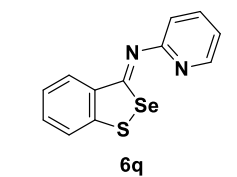

${ }^{1} \mathrm{H} \mathrm{NMR}\left(400 \mathrm{MHz}, \mathrm{CDCl}_{3}\right)$

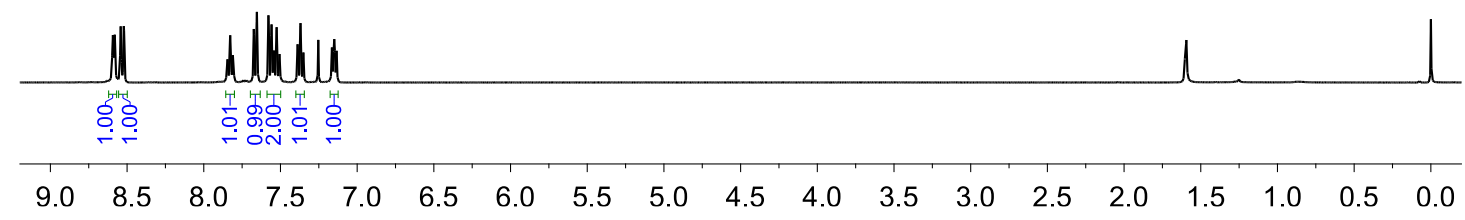

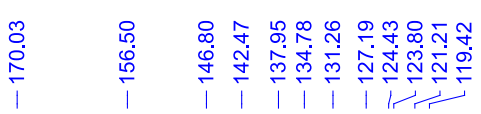

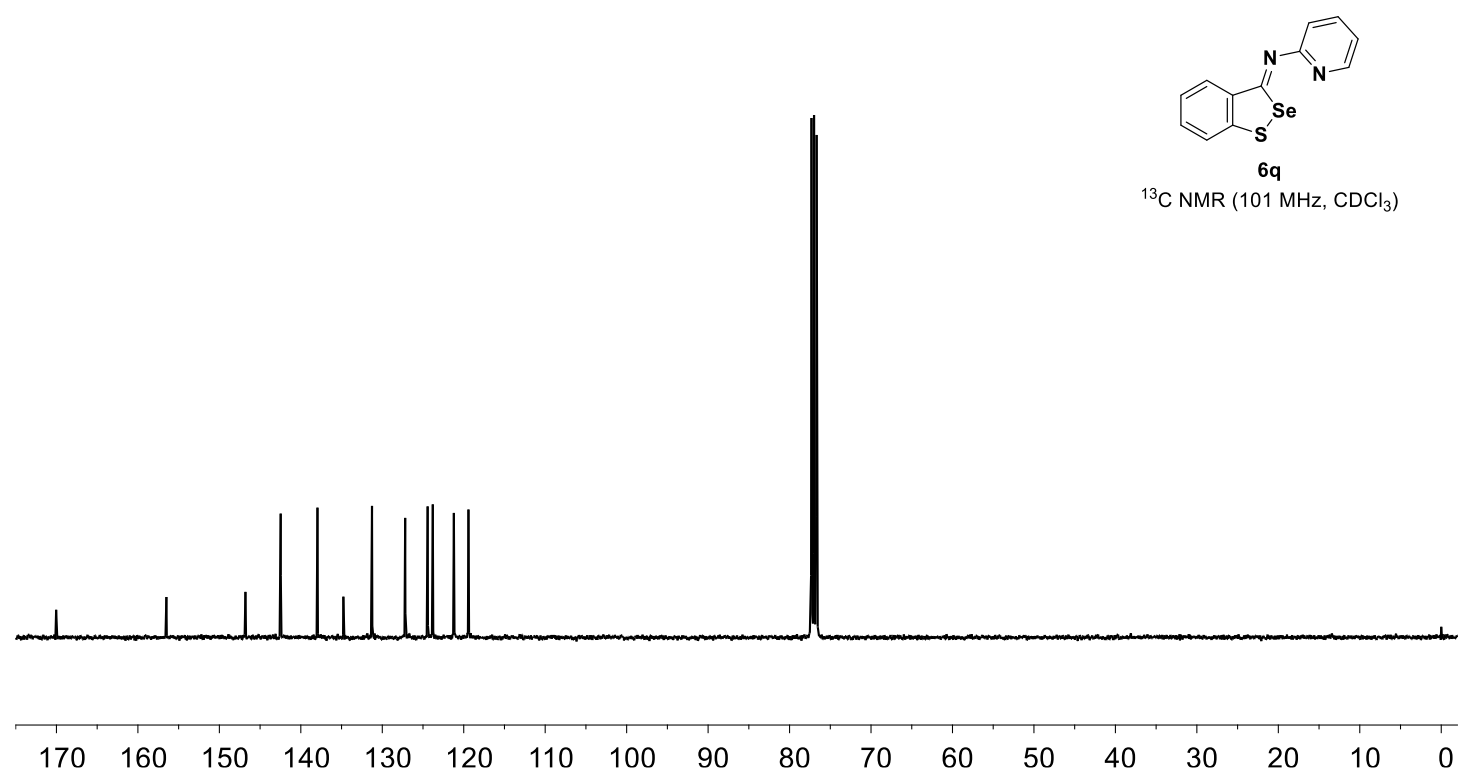




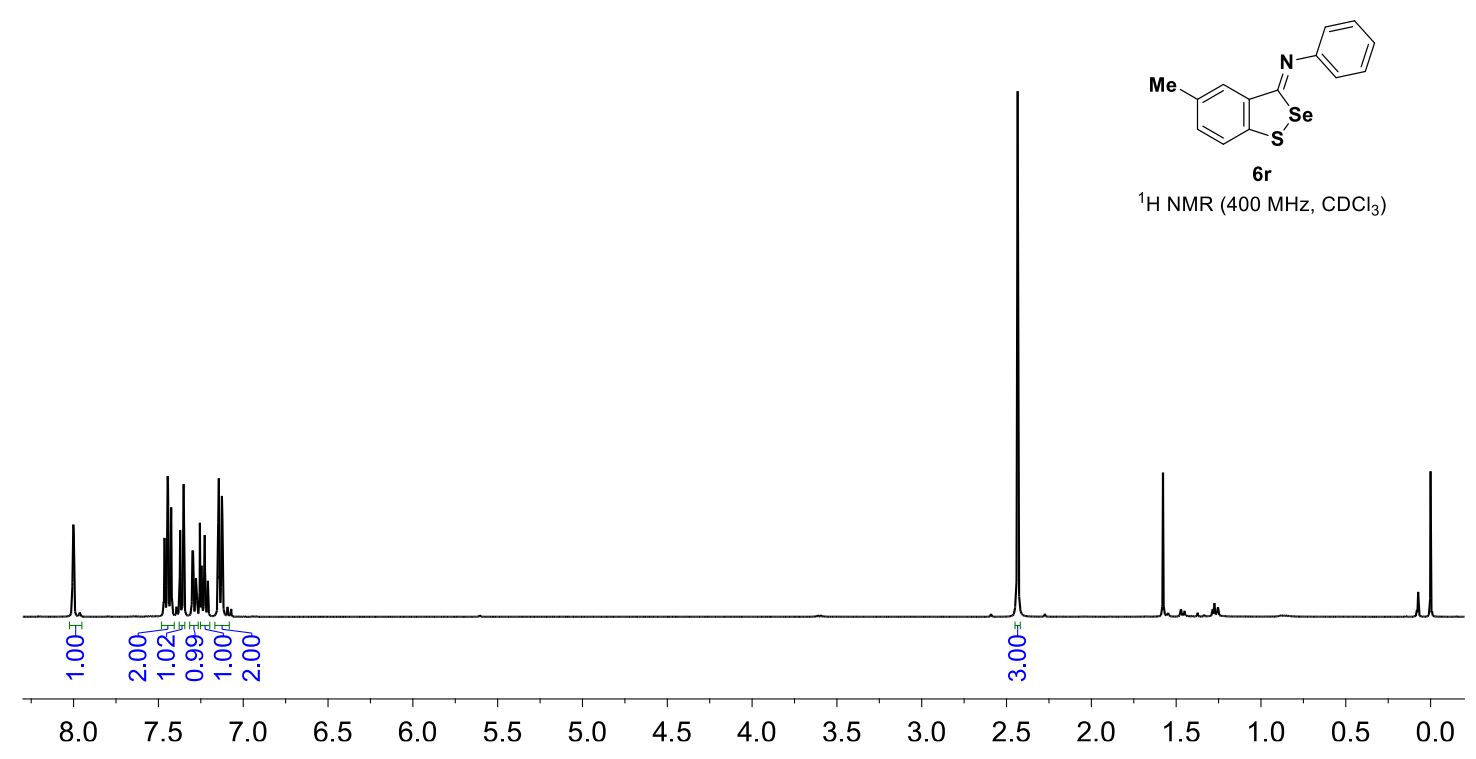

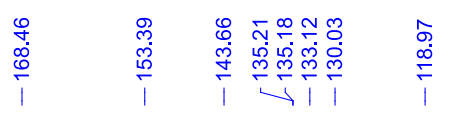
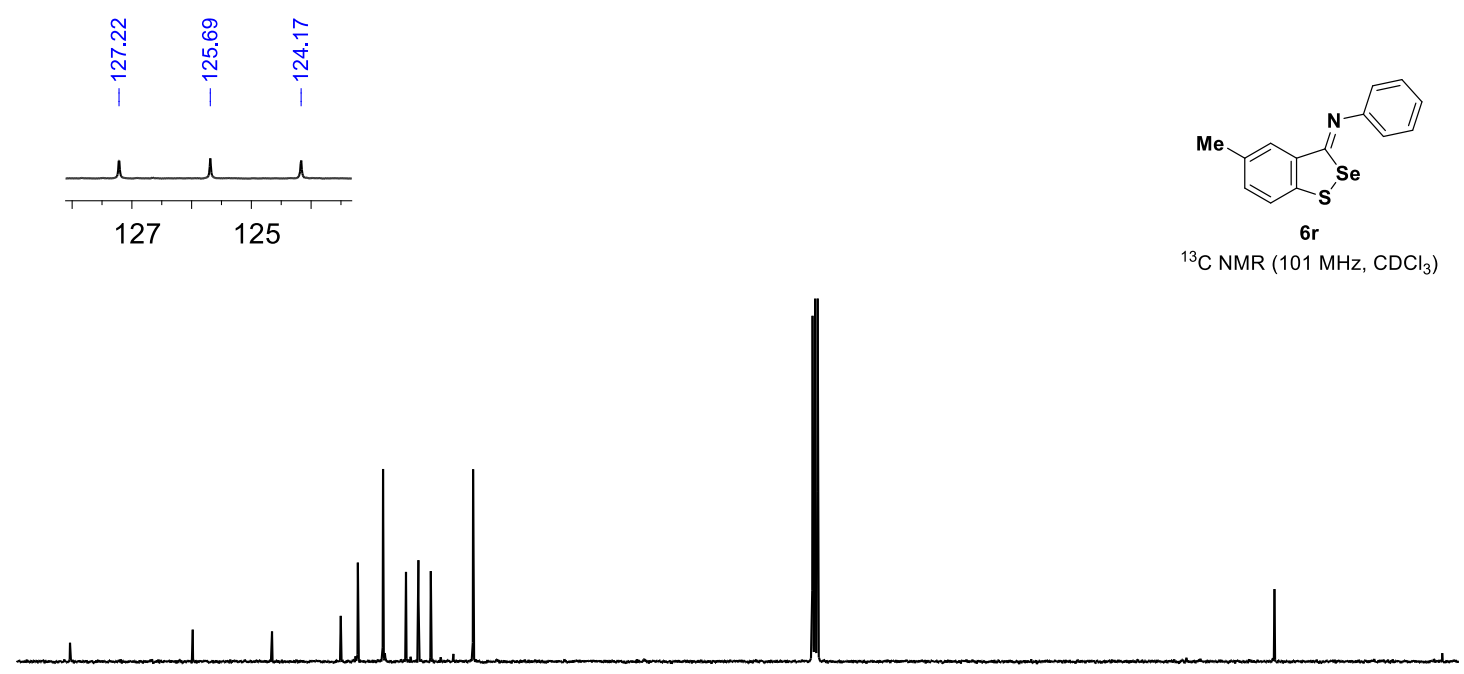

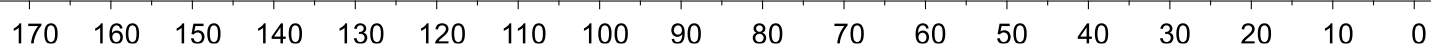




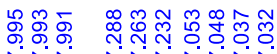

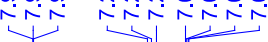

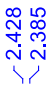

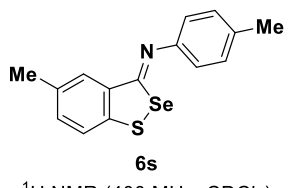

${ }^{1} \mathrm{H} \mathrm{NMR}\left(400 \mathrm{MHz}, \mathrm{CDCl}_{3}\right)$
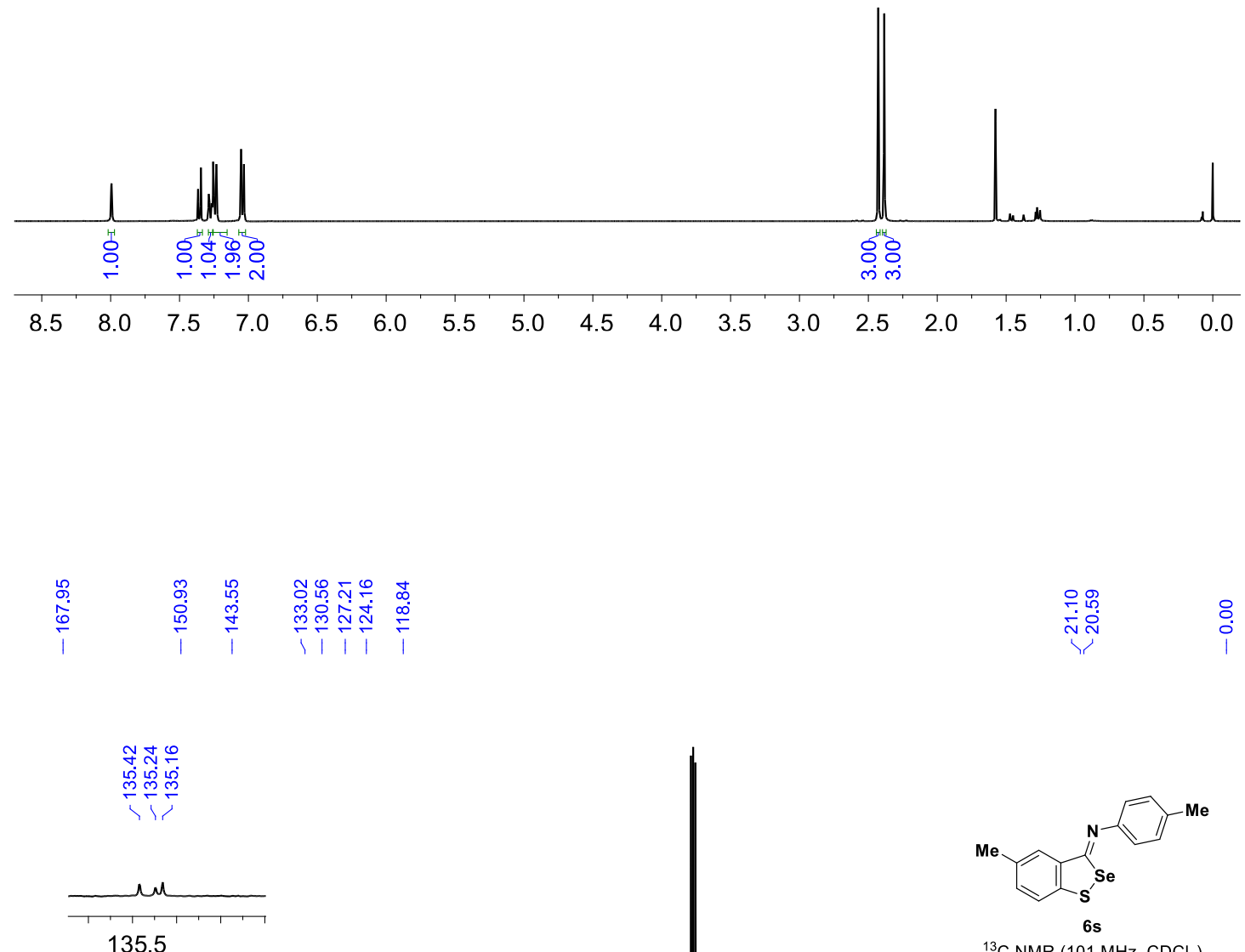

${ }^{13} \mathrm{C}$ NMR $\left(101 \mathrm{MHz}, \mathrm{CDCl}_{3}\right)$

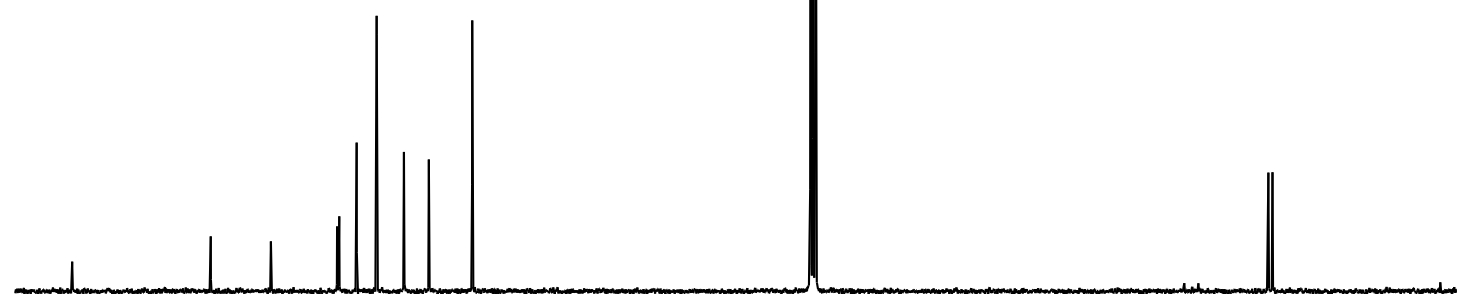

$\begin{array}{llllllllllllllllll}170 & 160 & 150 & 140 & 130 & 120 & 110 & 100 & 90 & 80 & 70 & 60 & 50 & 40 & 30 & 20 & 10 & 0\end{array}$ 


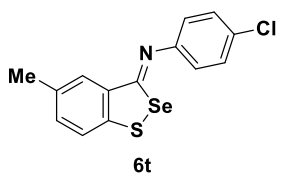

${ }^{1} \mathrm{H} \mathrm{NMR}\left(400 \mathrm{MHz}, \mathrm{CDCl}_{3}\right)$

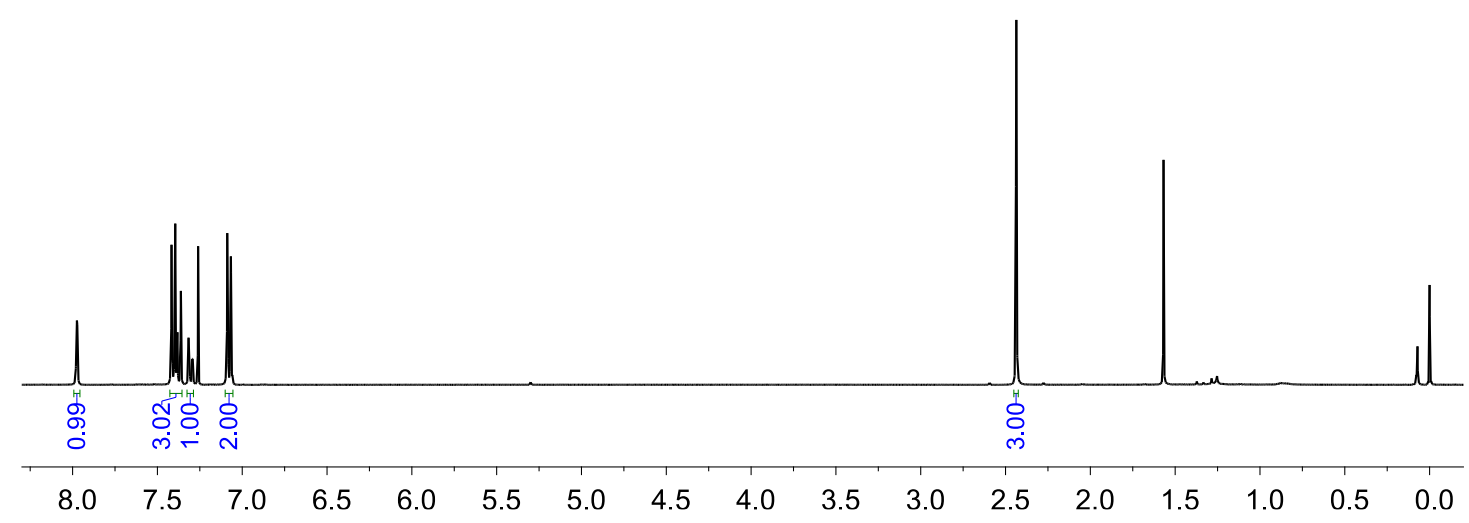

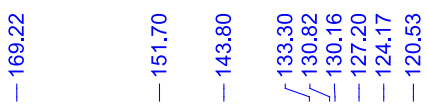

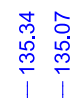

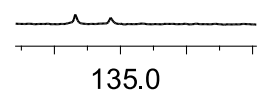

135.0

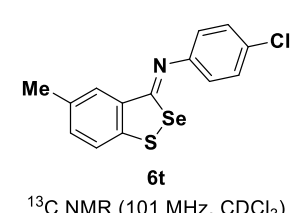

${ }^{13} \mathrm{C}$ NMR $\left(101 \mathrm{MHz}, \mathrm{CDCl}_{3}\right)$

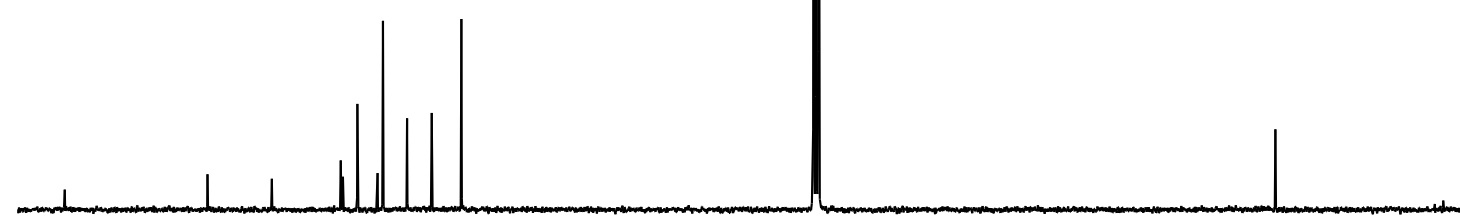

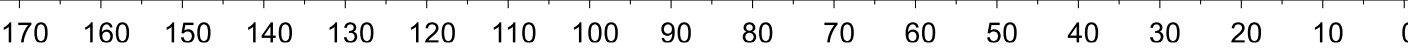




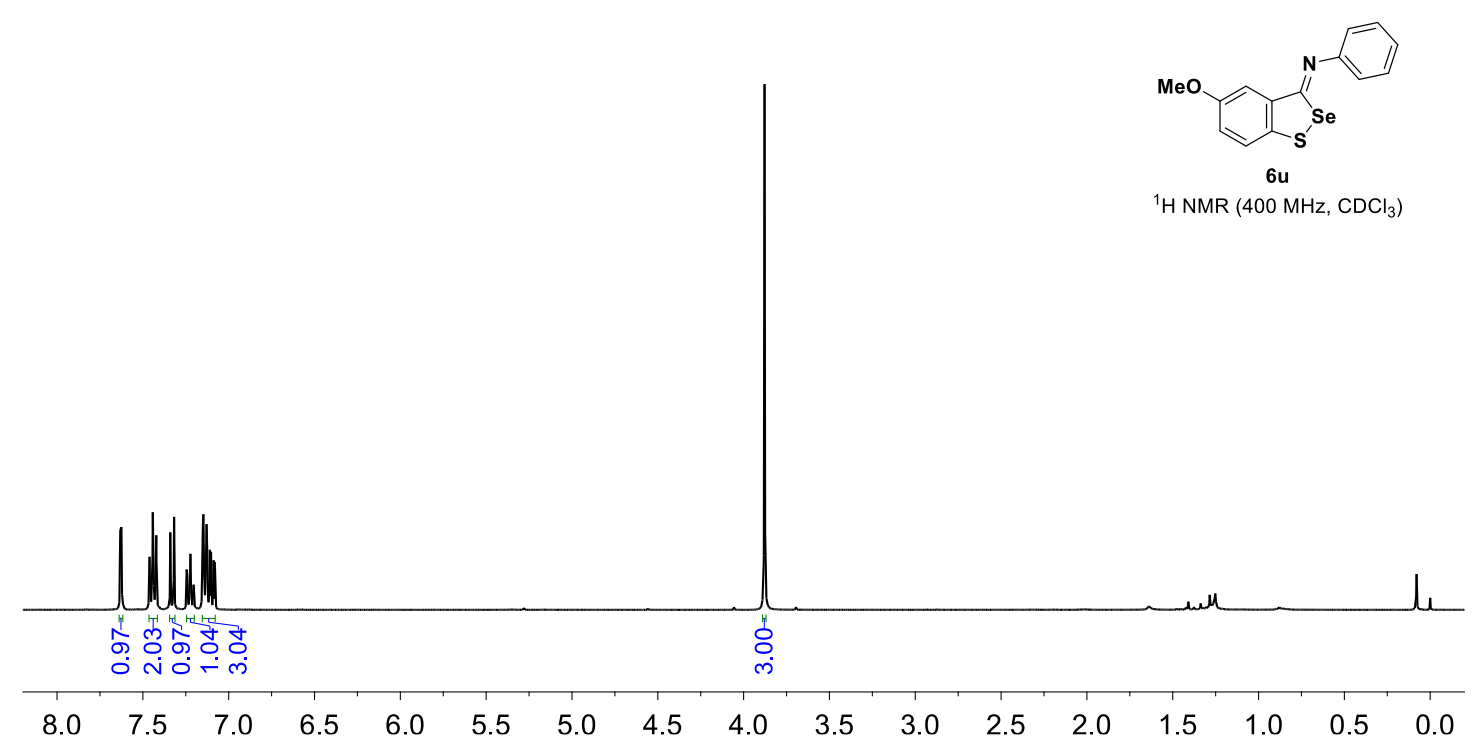

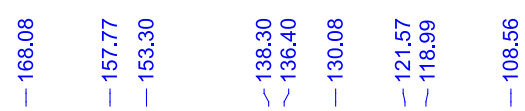

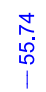
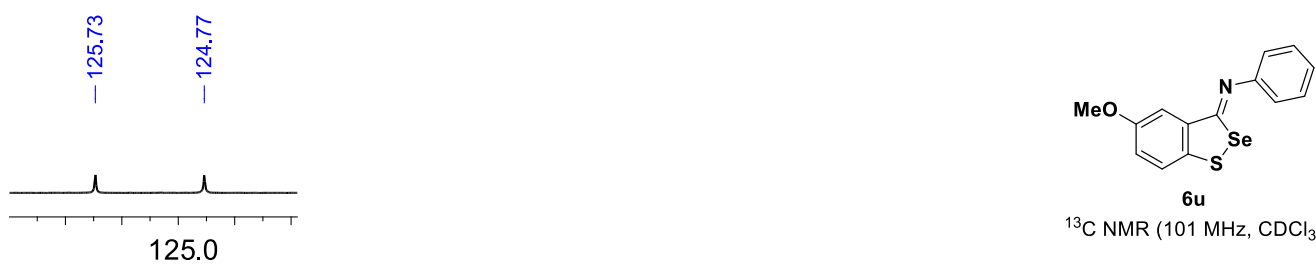

${ }^{13} \mathrm{C}$ NMR (101 MHz, $\left.\mathrm{CDCl}_{3}\right)$

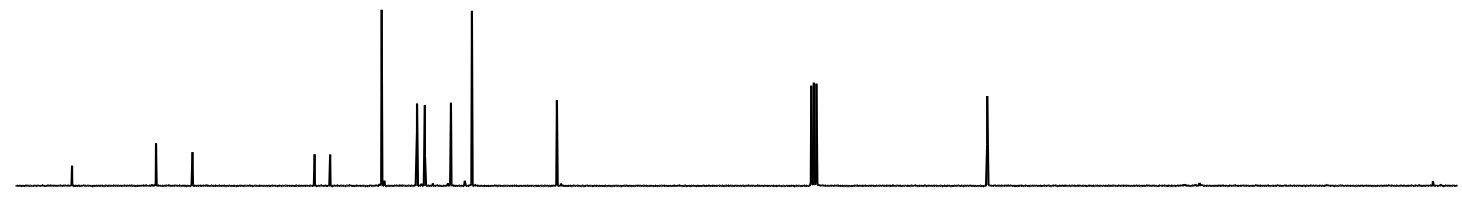

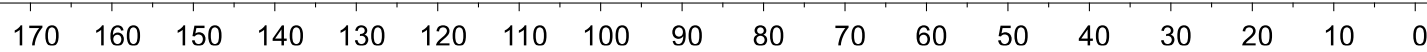



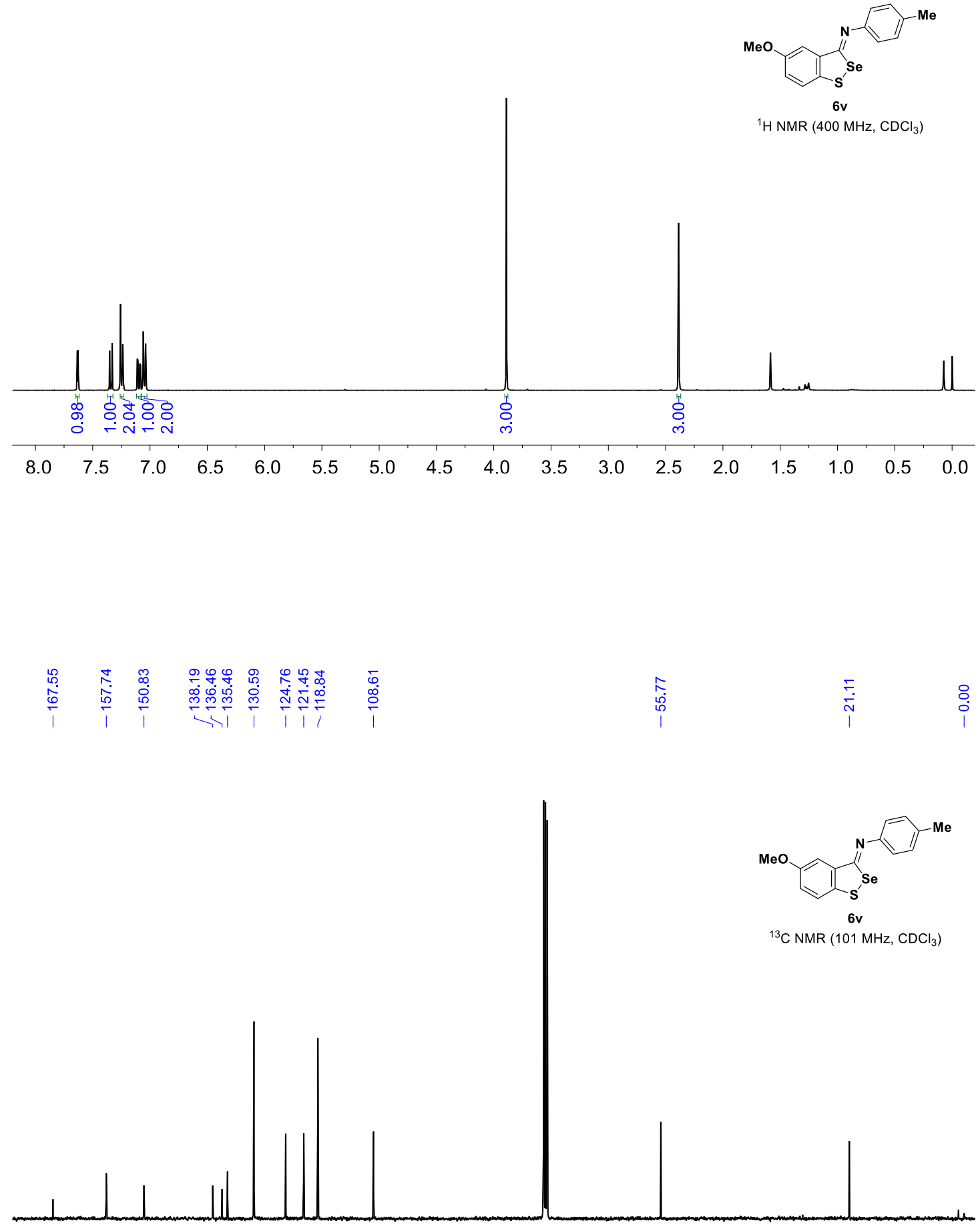

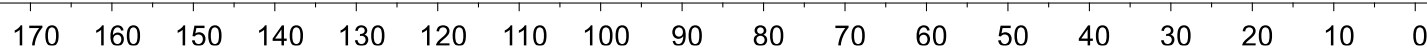




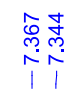

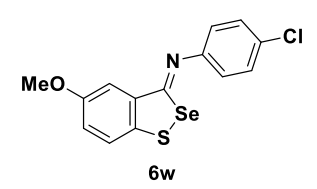

${ }^{1} \mathrm{H}$ NMR (400 MHz, $\left.\mathrm{CDCl}_{3}\right)$

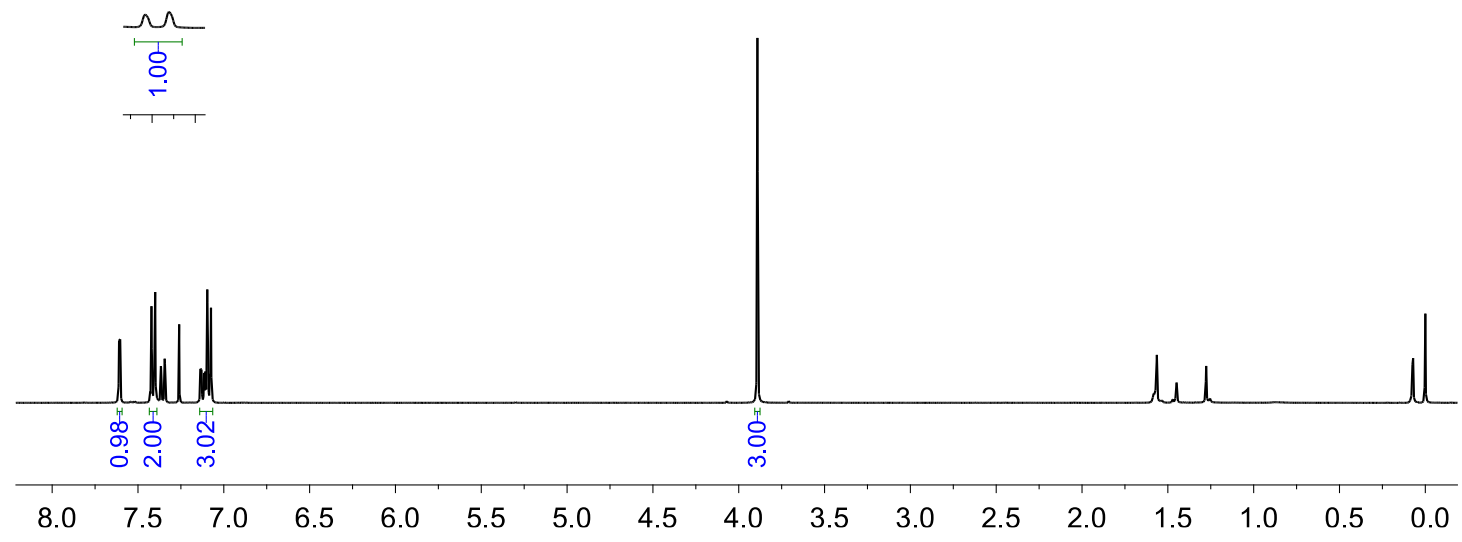

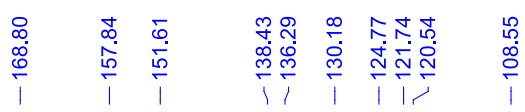

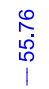
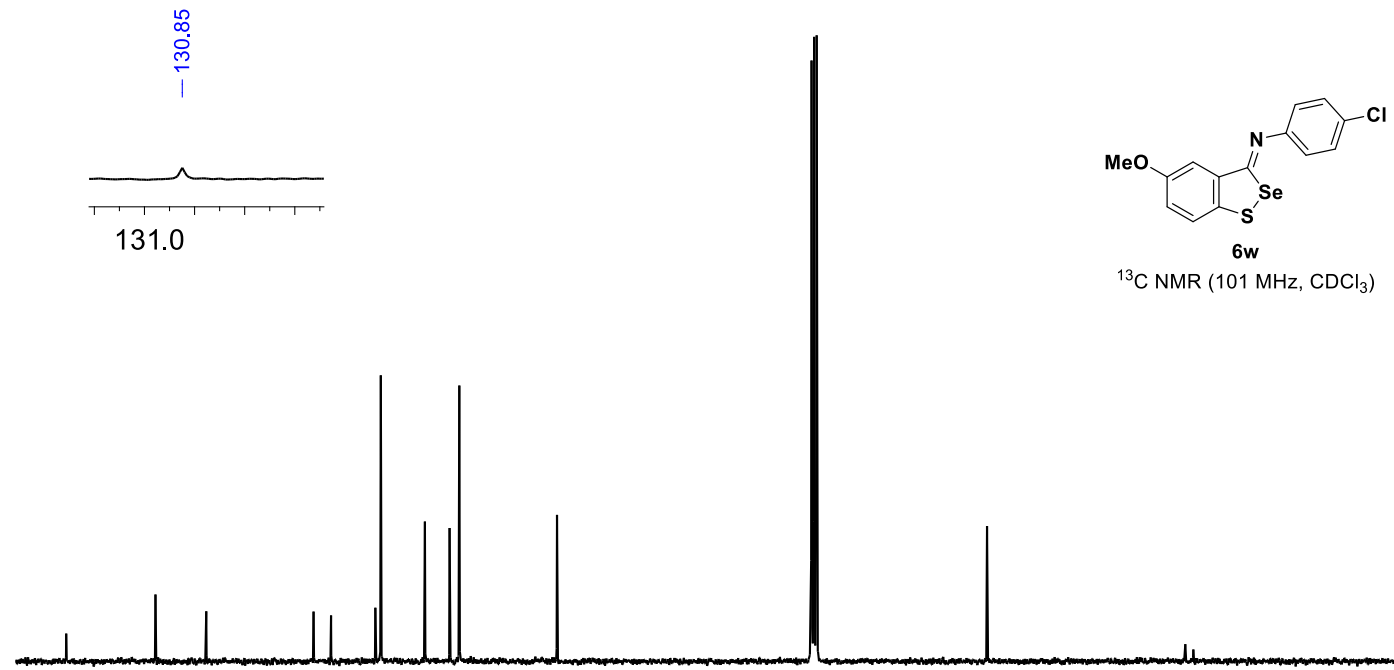

${ }^{13} \mathrm{C}$ NMR $\left(101 \mathrm{MHz}, \mathrm{CDCl}_{3}\right)$

$\begin{array}{llllllllllllllllll}170 & 160 & 150 & 140 & 130 & 120 & 110 & 100 & 90 & 80 & 70 & 60 & 50 & 40 & 30 & 20 & 10 & 0\end{array}$ 


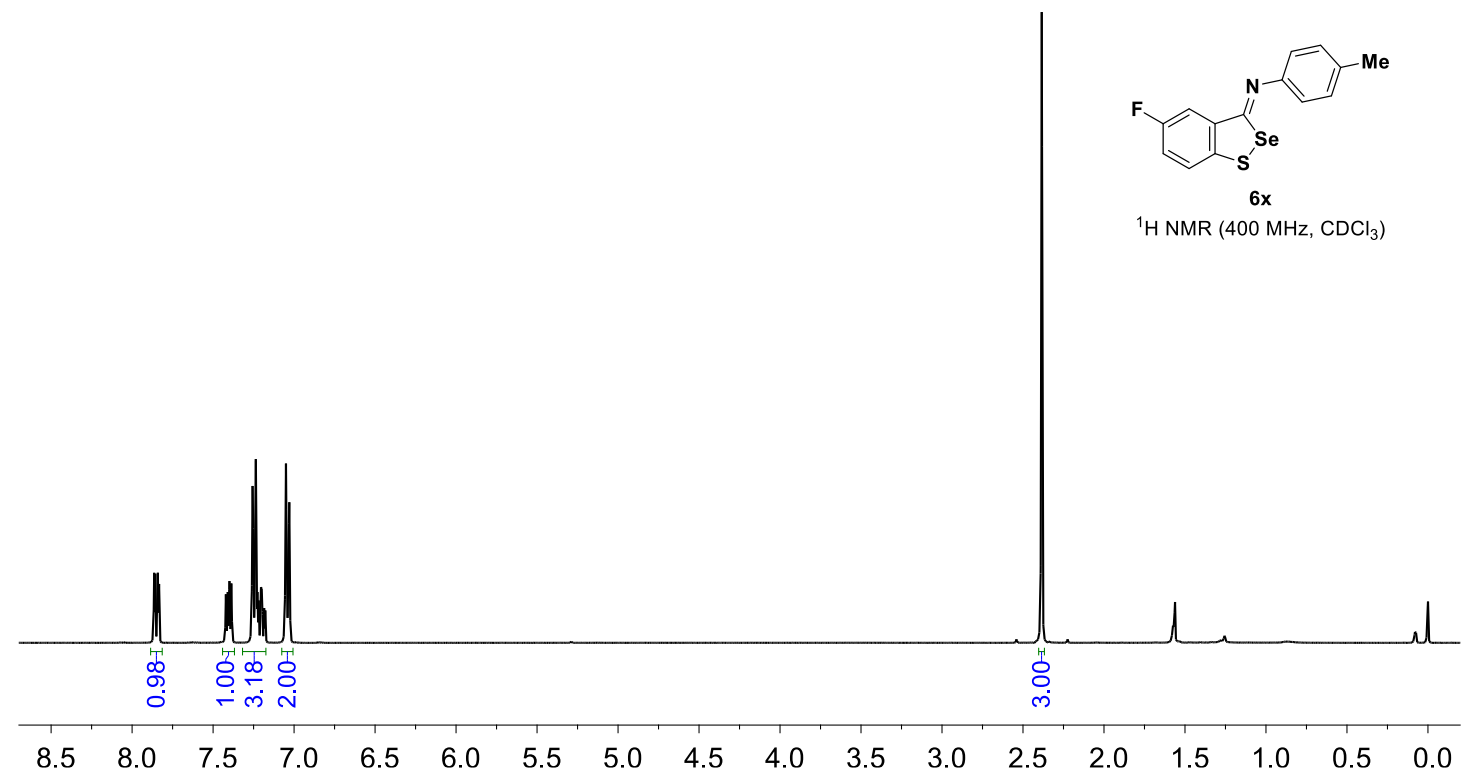

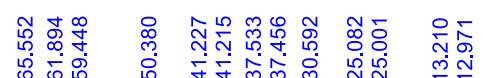

।

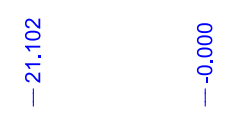

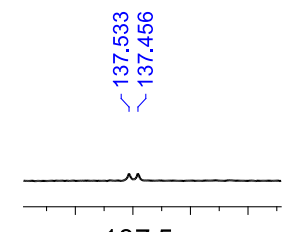

137.5

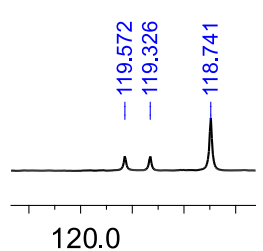

120.0

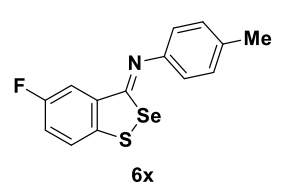

${ }^{13} \mathrm{C}$ NMR (101 MHz, $\mathrm{CDCl}_{3}$ )

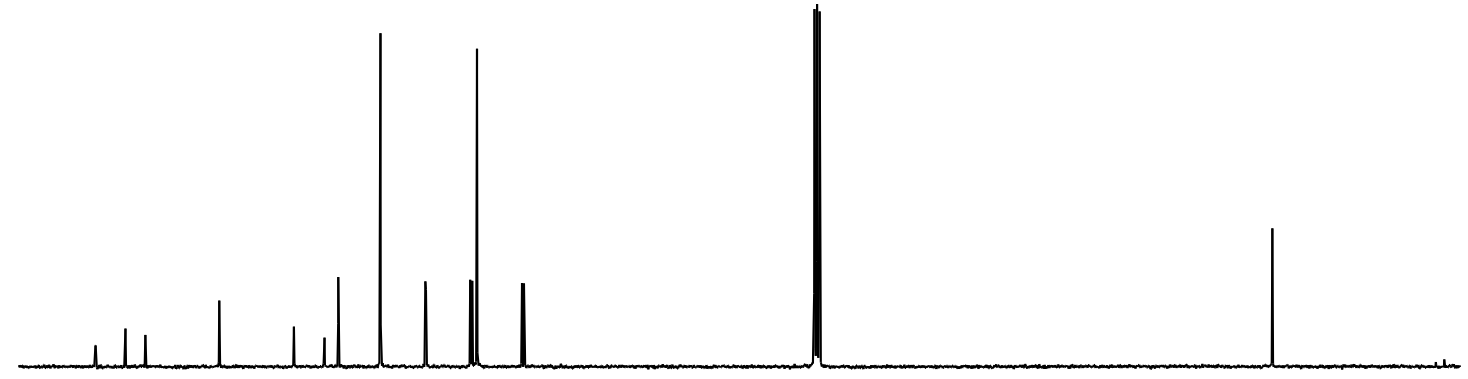

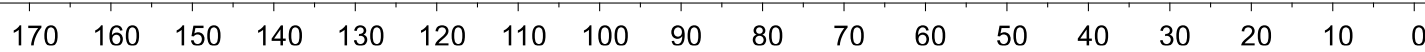



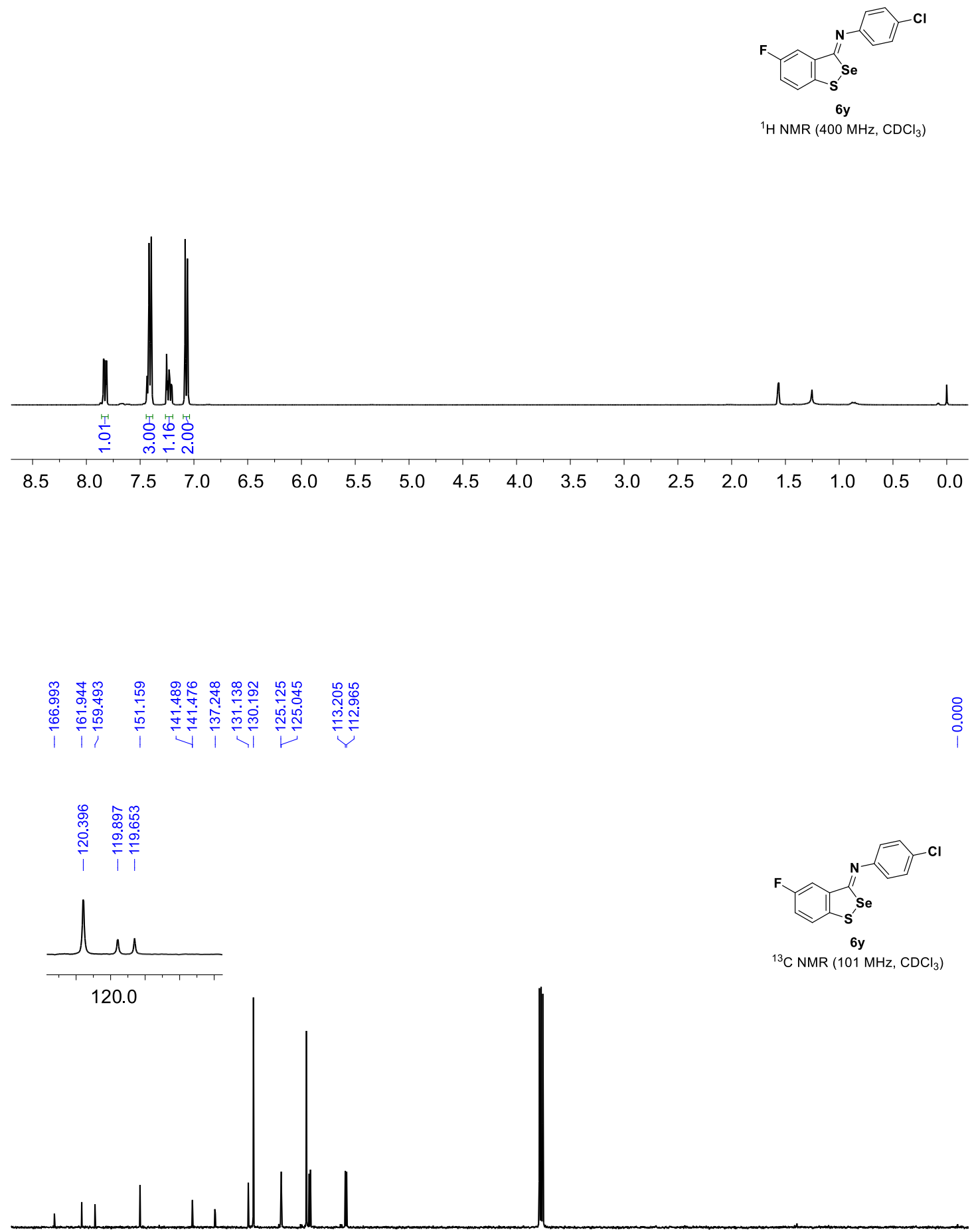

$\begin{array}{llllllllllllllllll}170 & 160 & 150 & 140 & 130 & 120 & 110 & 100 & 90 & 80 & 70 & 60 & 50 & 40 & 30 & 20 & 10 & 0\end{array}$ 


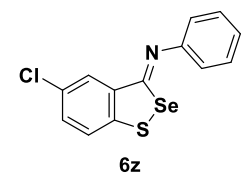

$\left.{ }^{1} \mathrm{H} \mathrm{NMR} \mathrm{(400} \mathrm{MHz,} \mathrm{CDCl}_{3}\right)$

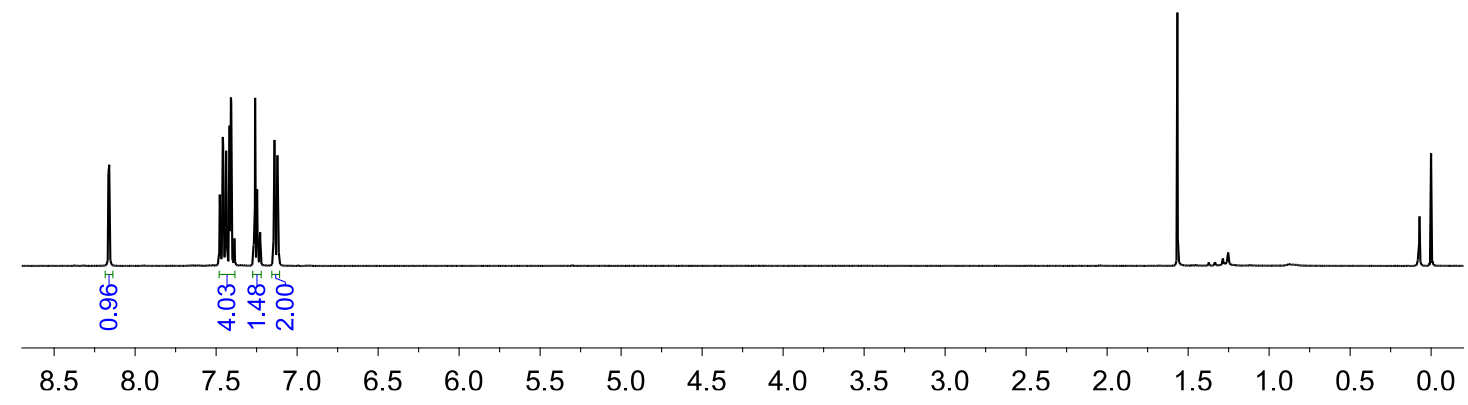

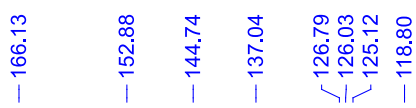

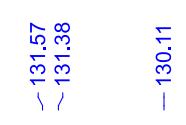

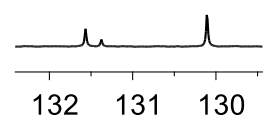

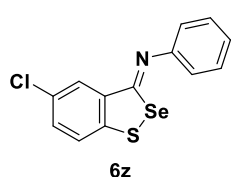

${ }^{13} \mathrm{C}$ NMR $\left(101 \mathrm{MHz}, \mathrm{CDCl}_{3}\right)$

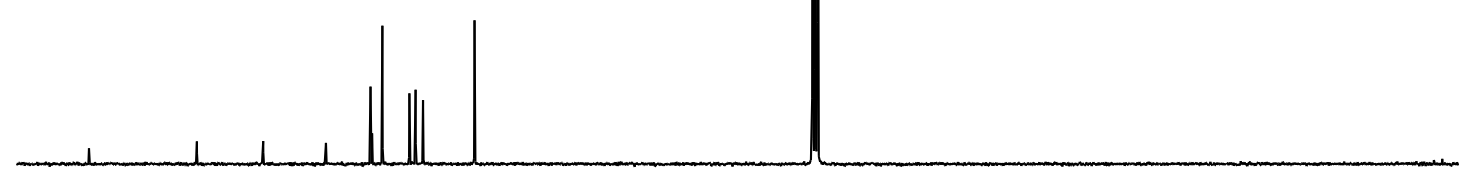

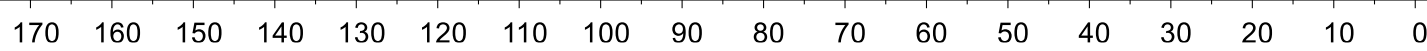




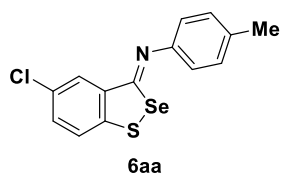

${ }^{1} \mathrm{H} \mathrm{NMR}\left(400 \mathrm{MHz}, \mathrm{CDCl}_{3}\right)$

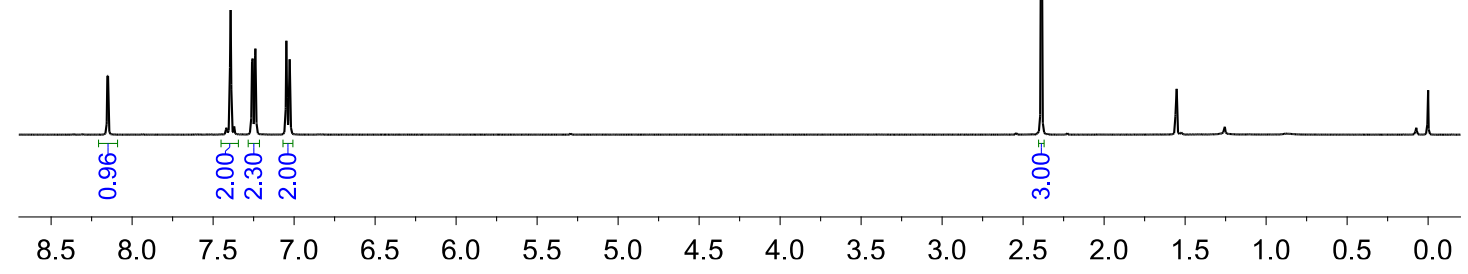

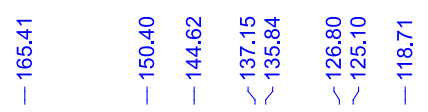
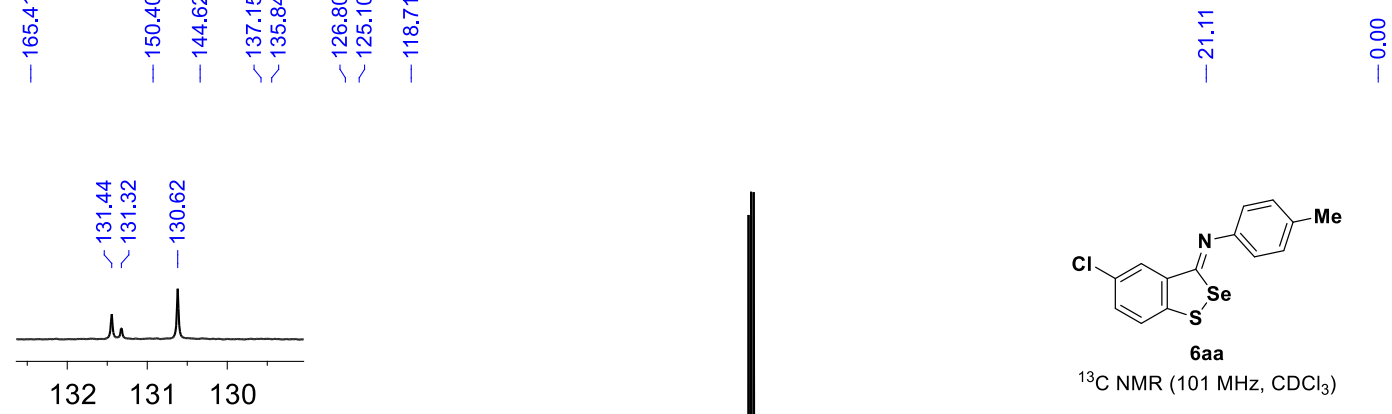

${ }^{13} \mathrm{C}$ NMR $\left(101 \mathrm{MHz}, \mathrm{CDCl}_{3}\right)$

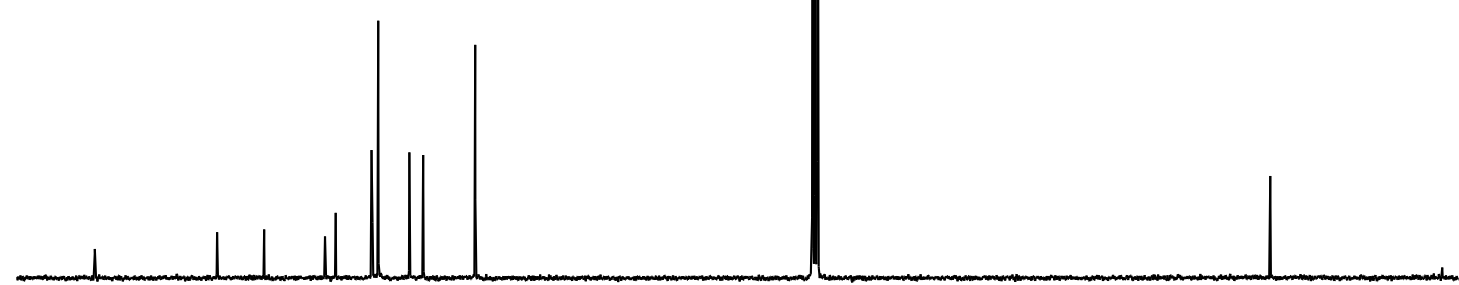

$\begin{array}{llllllllllllllllll}170 & 160 & 150 & 140 & 130 & 120 & 110 & 100 & 90 & 80 & 70 & 60 & 50 & 40 & 30 & 20 & 10 & 0\end{array}$ 


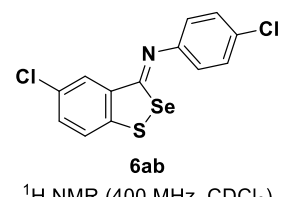

${ }^{1} \mathrm{H} \mathrm{NMR}\left(400 \mathrm{MHz}, \mathrm{CDCl}_{3}\right)$

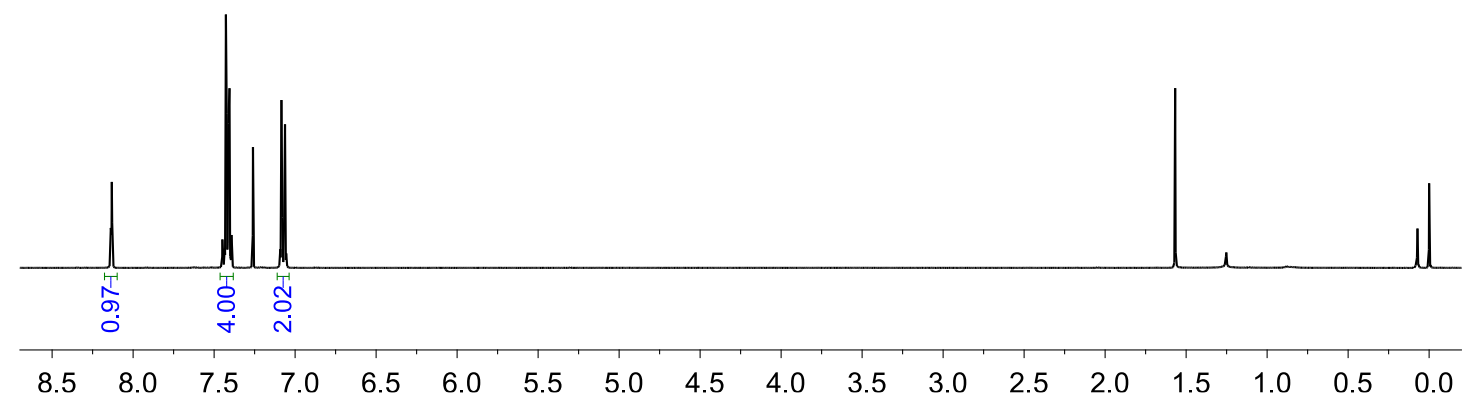

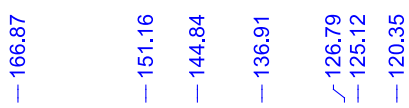
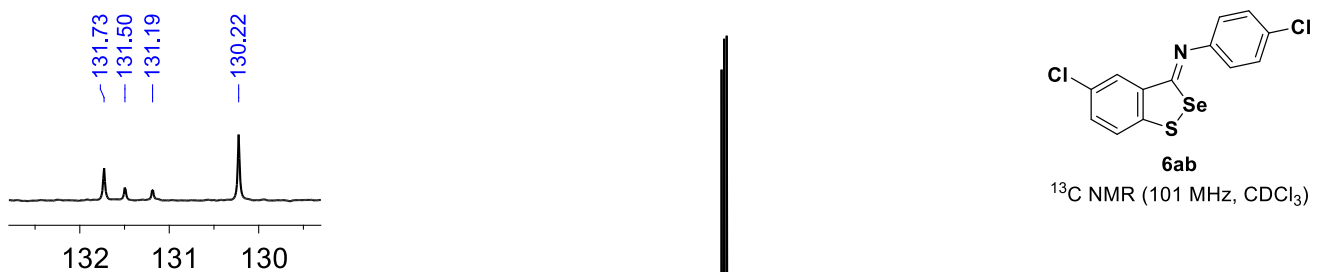

${ }^{13} \mathrm{C}$ NMR $\left(101 \mathrm{MHz}, \mathrm{CDCl}_{3}\right)$

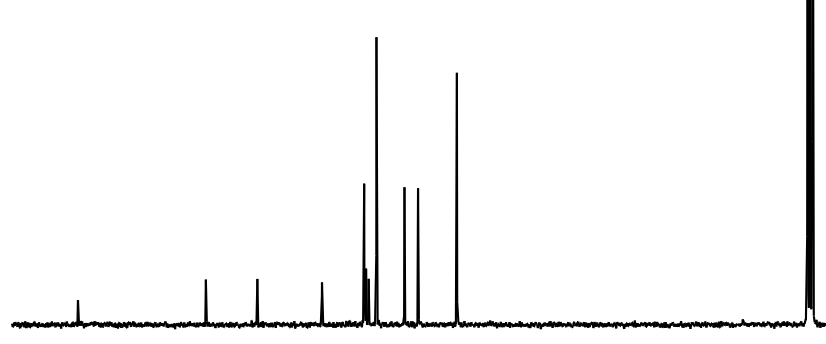

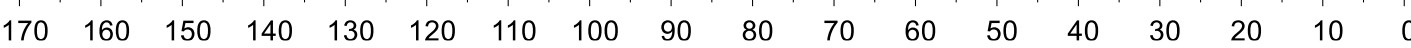



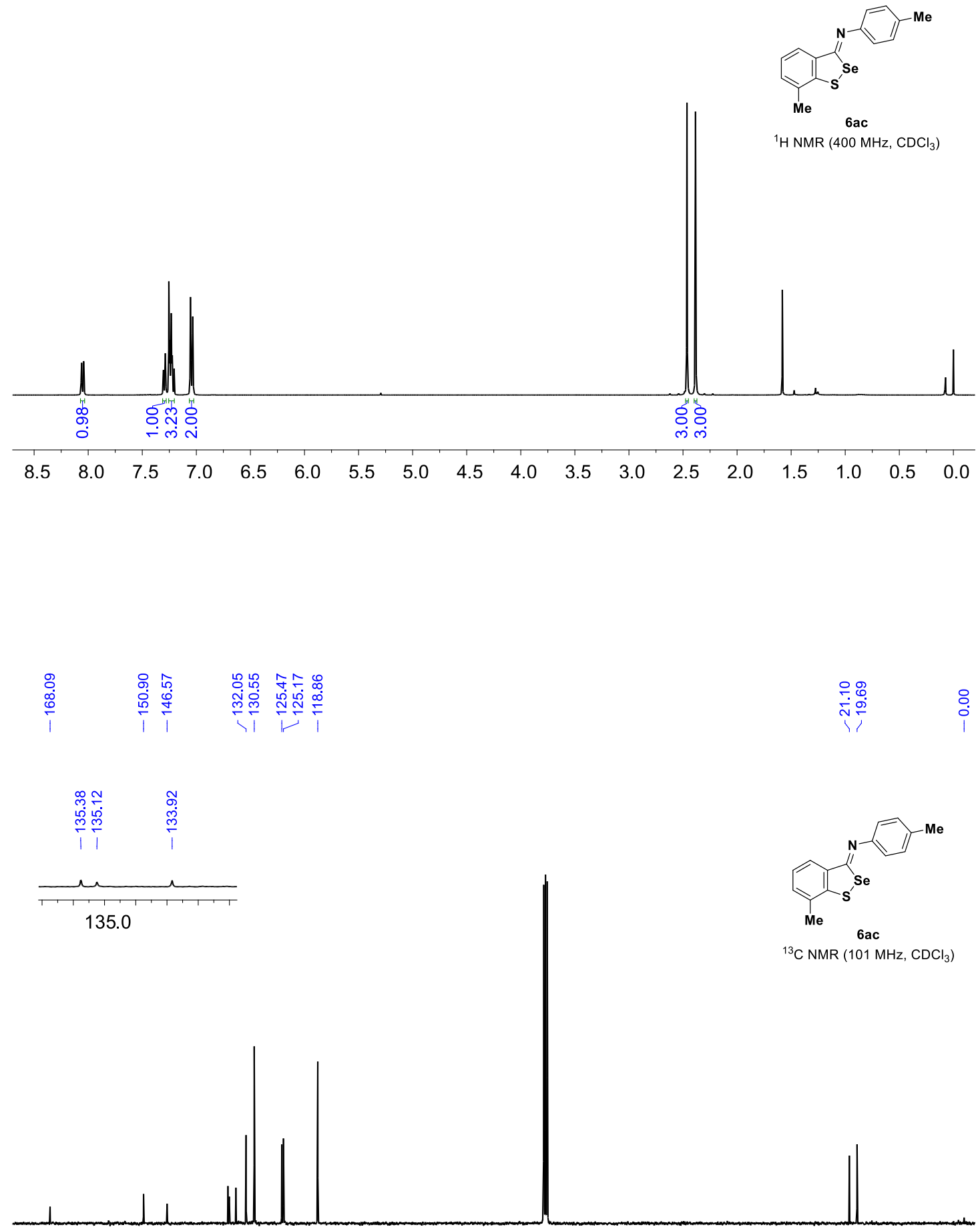

$\begin{array}{llllllllllllllllll}170 & 160 & 150 & 140 & 130 & 120 & 110 & 100 & 90 & 80 & 70 & 60 & 50 & 40 & 30 & 20 & 10 & 0\end{array}$ 


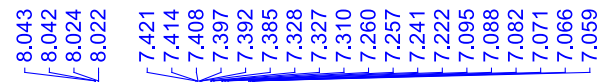

$\underset{\substack{\text { N } \\ \Gamma}}{1}$

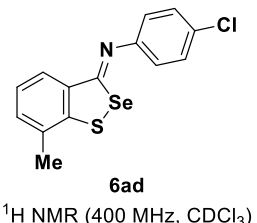

${ }^{1} \mathrm{H} \mathrm{NMR}\left(400 \mathrm{MHz}, \mathrm{CDCl}_{3}\right)$

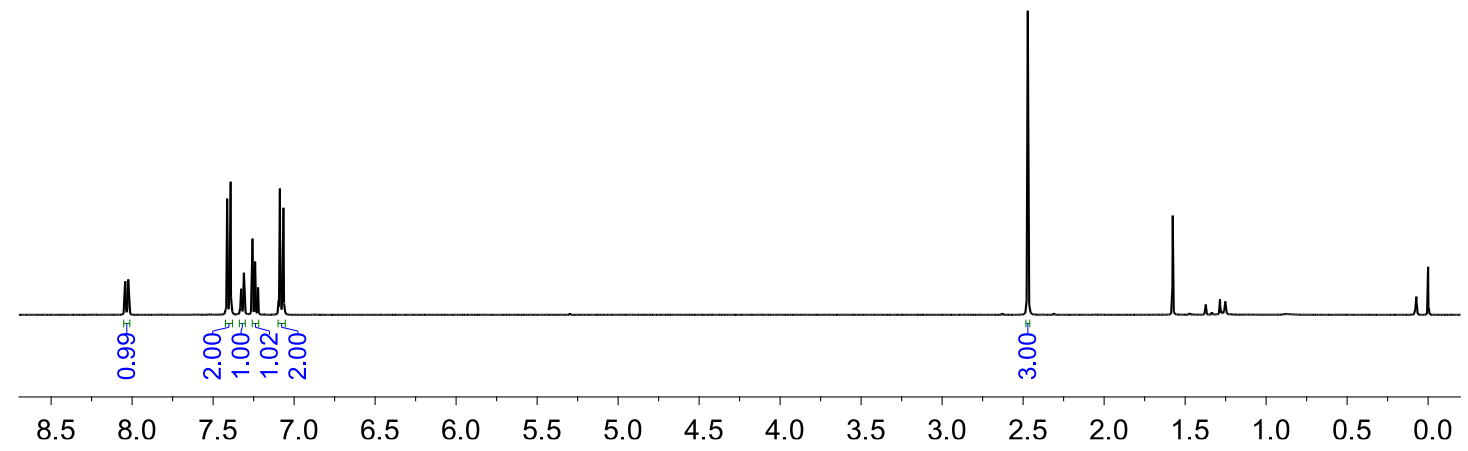

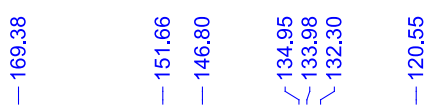
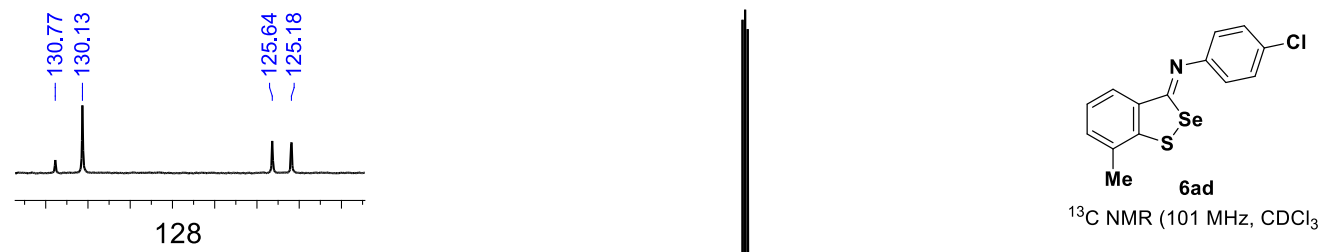

${ }^{13} \mathrm{C} \mathrm{NMR}\left(101 \mathrm{MHz}, \mathrm{CDCl}_{3}\right)$

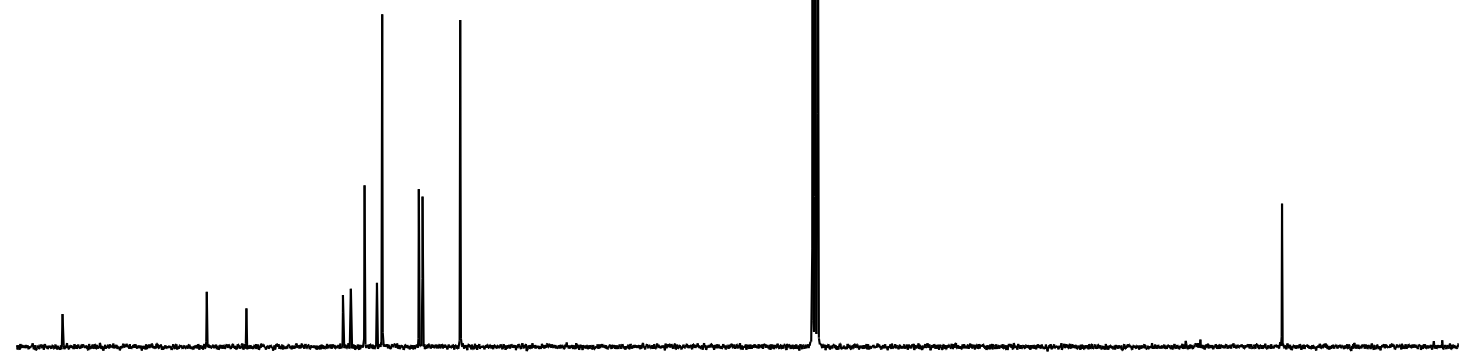

$\begin{array}{lllllllllllllllll}170 & 160 & 150 & 140 & 130 & 120 & 110 & 100 & 90 & 80 & 70 & 60 & 50 & 40 & 30 & 20 & 10\end{array}$ 


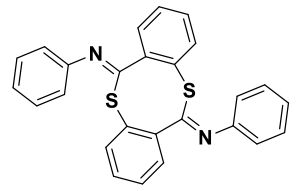

${ }^{1} \mathrm{H} \mathrm{NMR}\left(400 \mathrm{MHz}, \mathrm{CDCl}_{3}\right)$

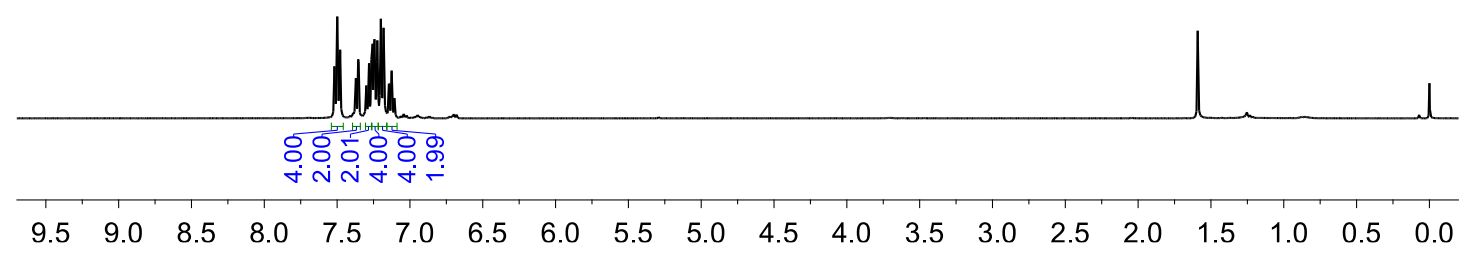

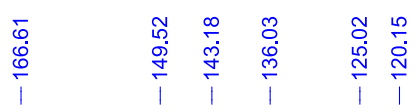

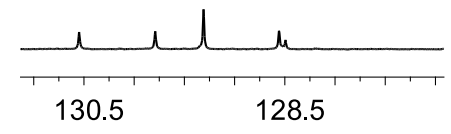

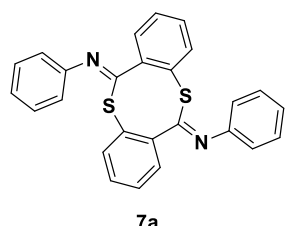

${ }^{13} \mathrm{C}$ NMR $\left(101 \mathrm{MHz}, \mathrm{CDCl}_{3}\right)$

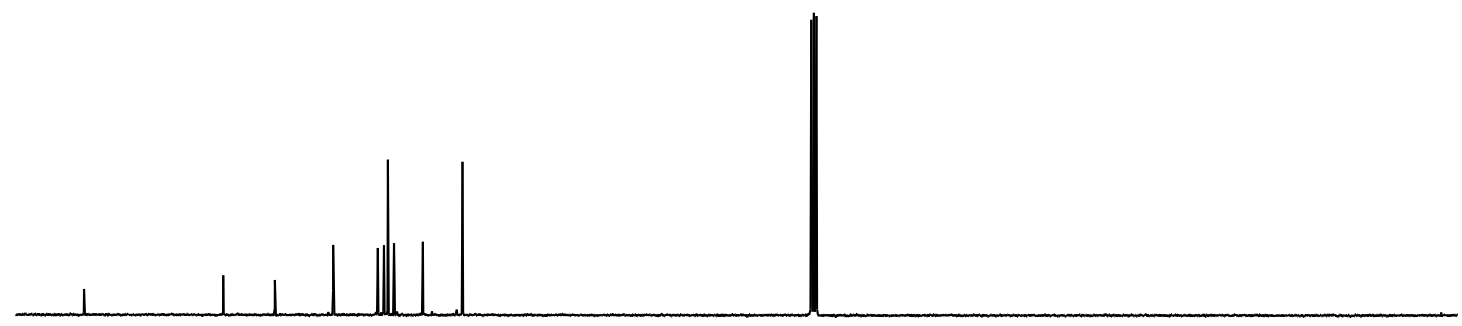

$\begin{array}{lllllllllllllllllll}170 & 160 & 150 & 140 & 130 & 120 & 110 & 100 & 90 & 80 & 70 & 60 & 50 & 40 & 30 & 20 & 10 & 0\end{array}$ 


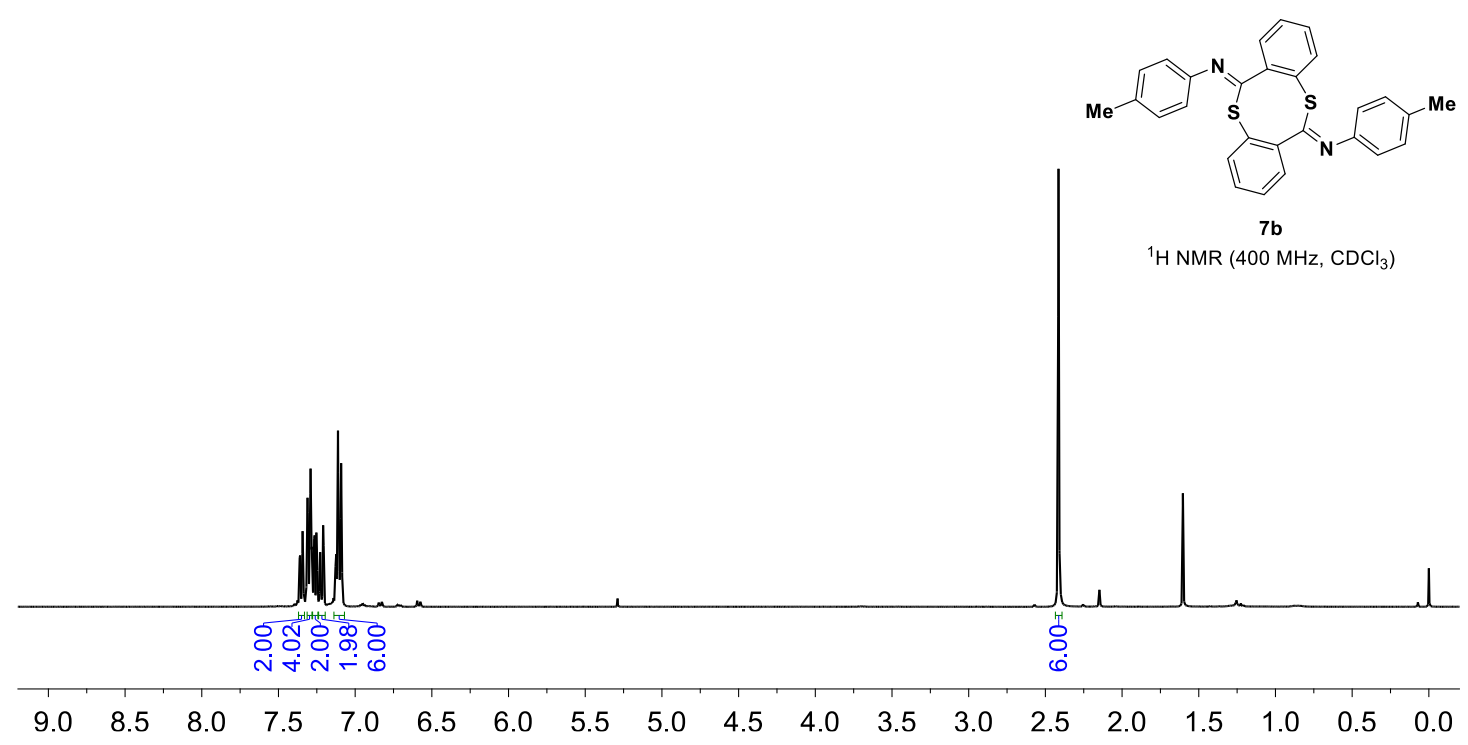

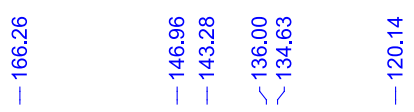

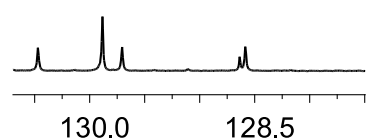

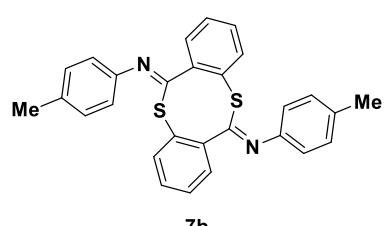

${ }^{13} \mathrm{C} \mathrm{NMR}\left(101 \mathrm{MHz}, \mathrm{CDCl}_{3}\right)$

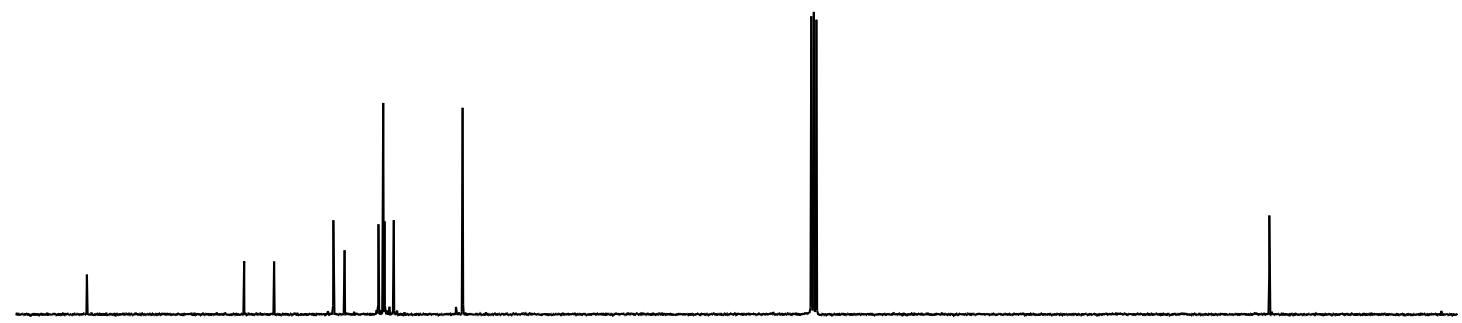

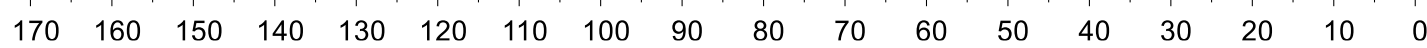




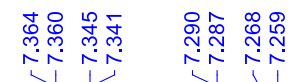
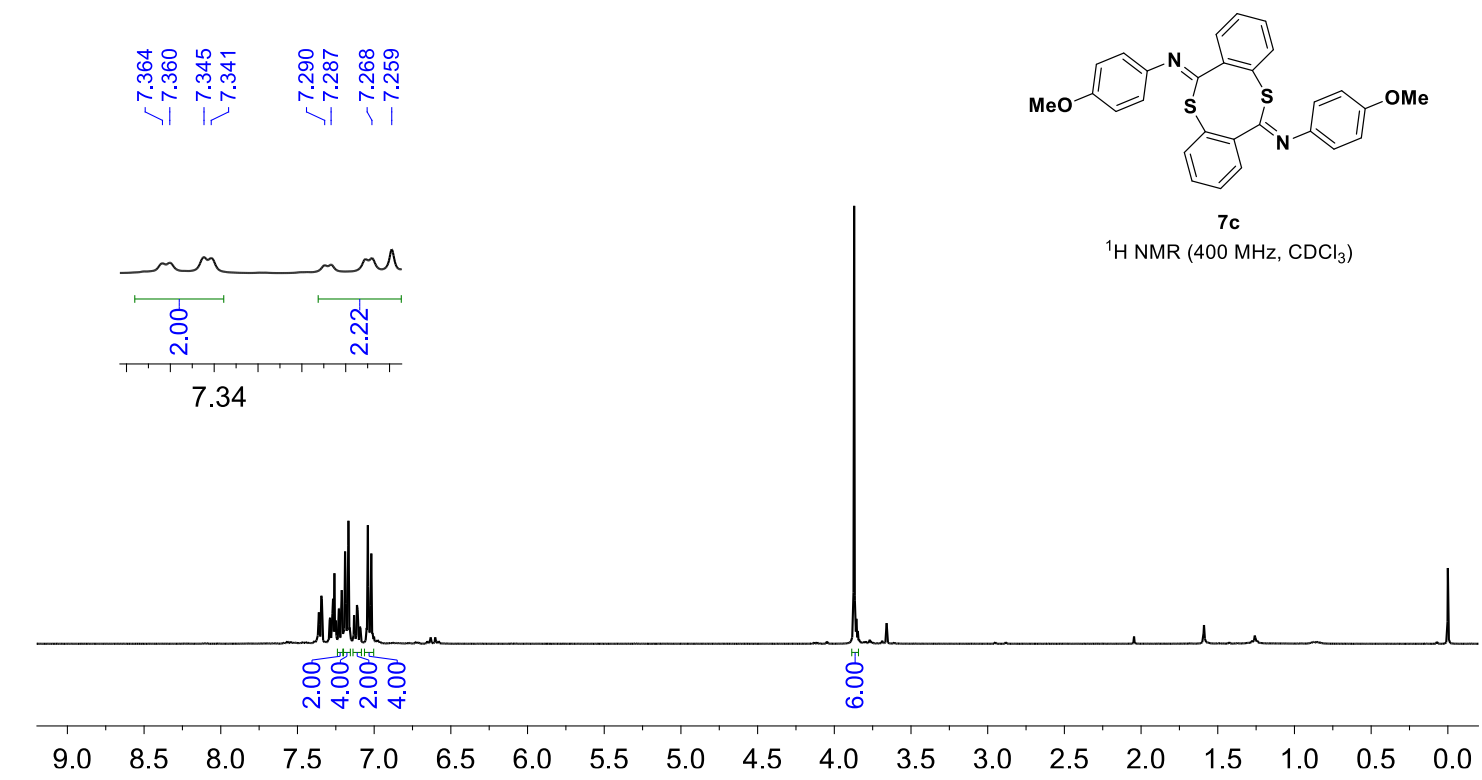

${ }^{1} \mathrm{H} \mathrm{NMR}\left(400 \mathrm{MHz}, \mathrm{CDCl}_{3}\right)$

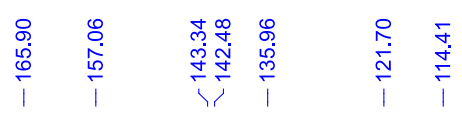
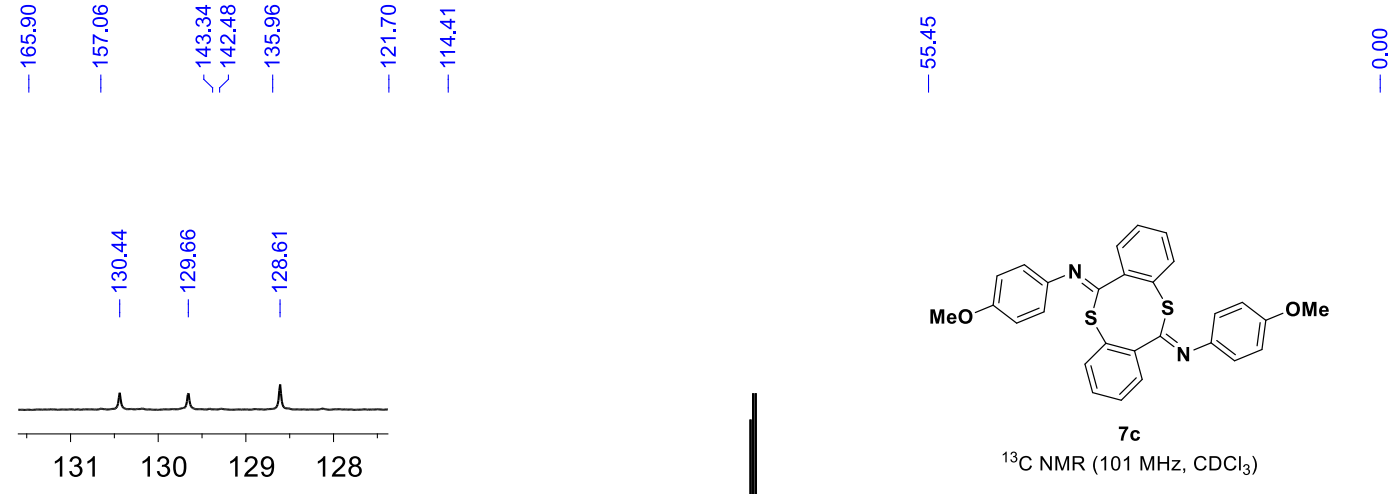

$\left.{ }^{13} \mathrm{C} \mathrm{NMR} \mathrm{(101} \mathrm{MHz,} \mathrm{CDCl}\right)$

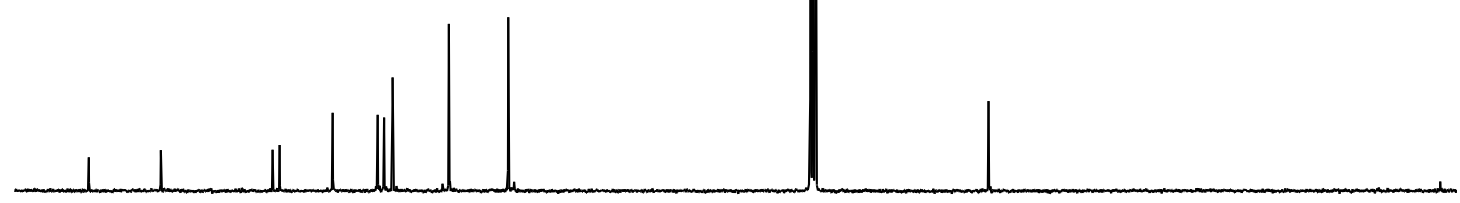

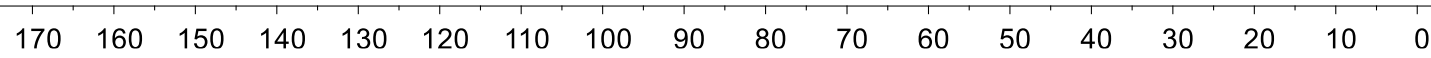



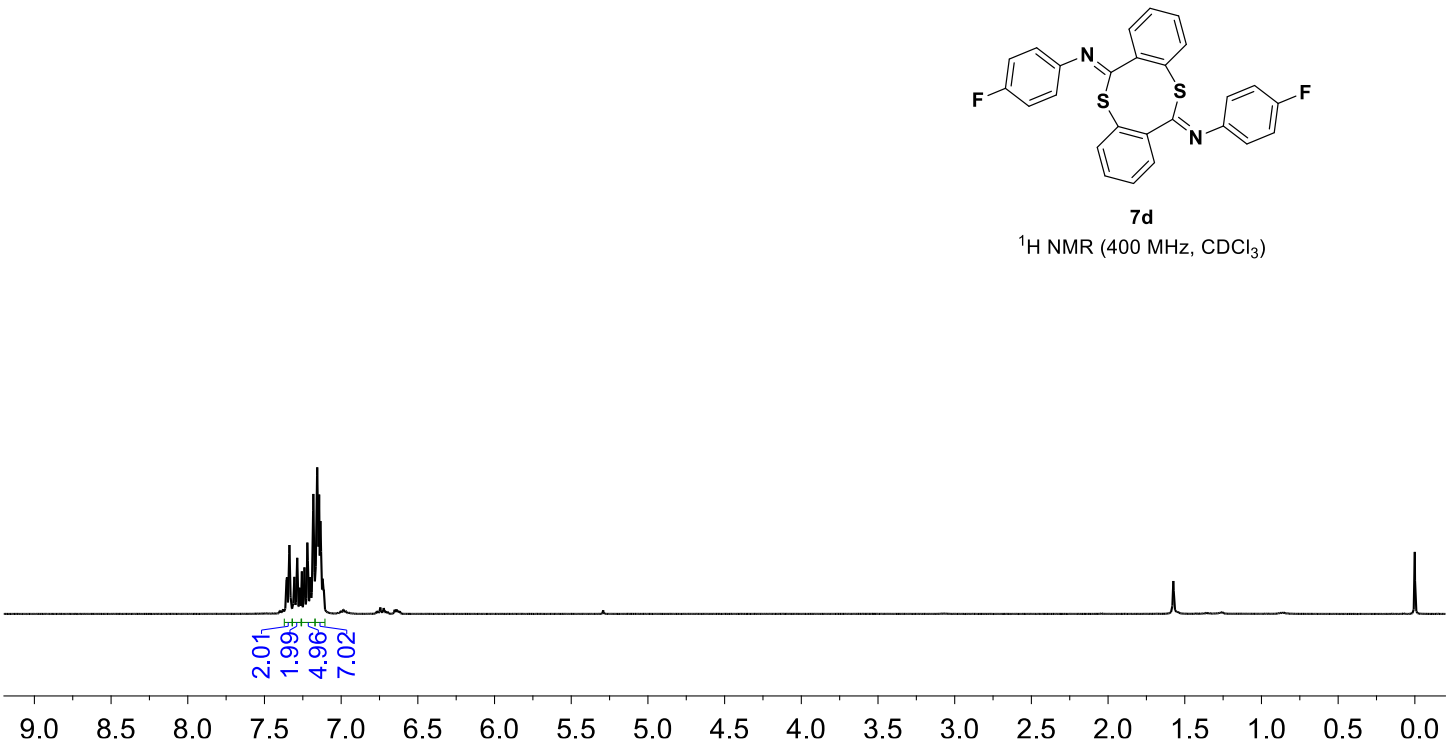

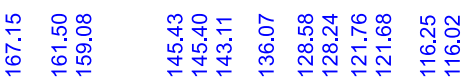
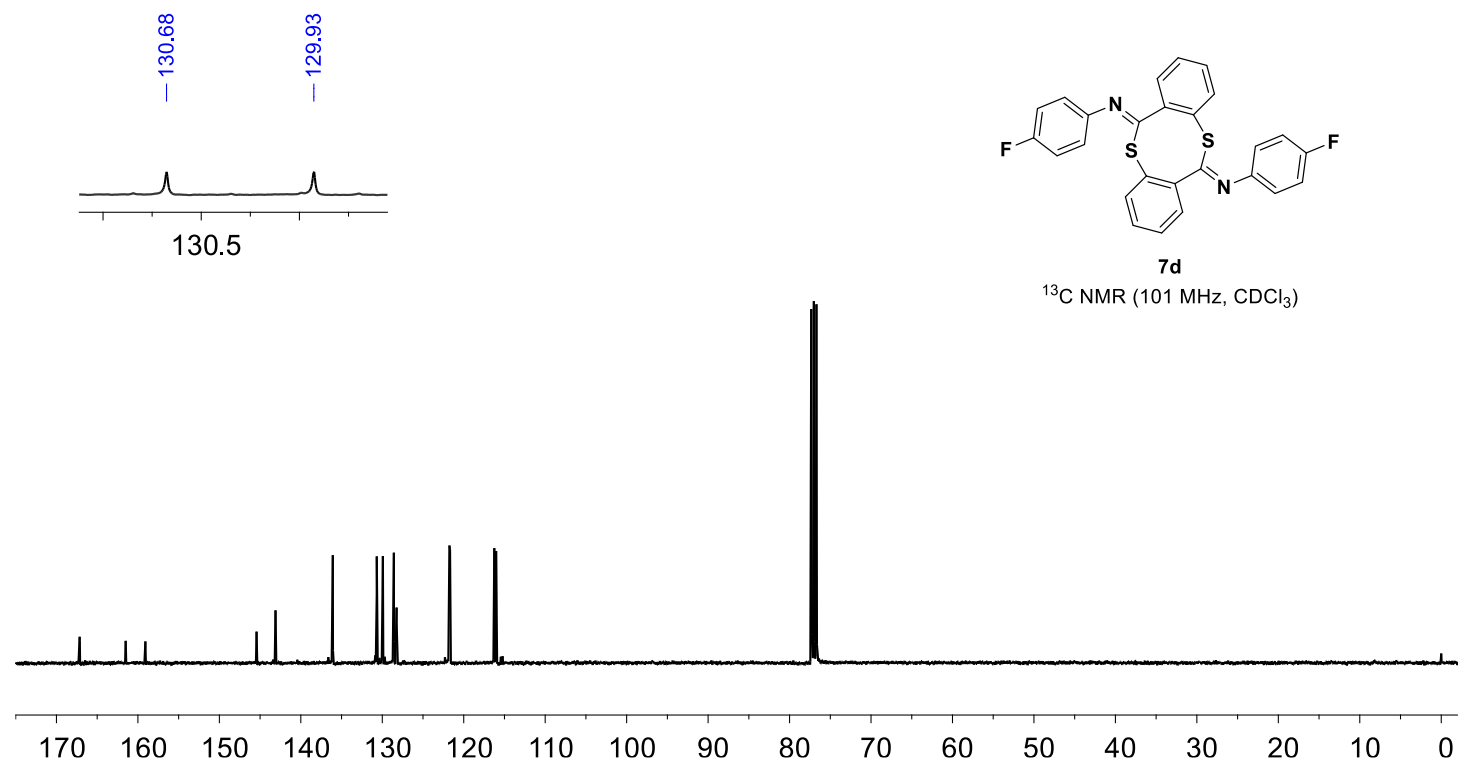


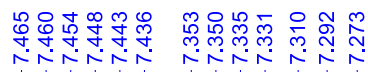
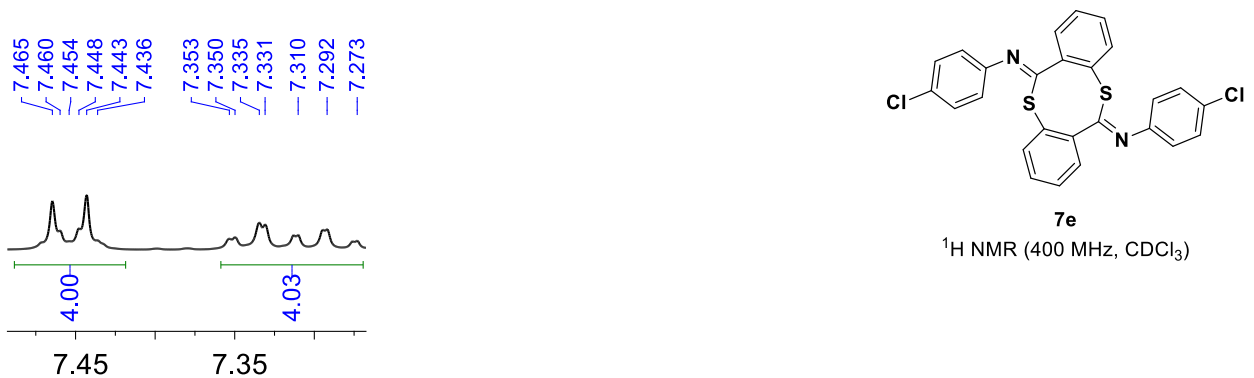

${ }^{1} \mathrm{H} \mathrm{NMR}\left(400 \mathrm{MHz}, \mathrm{CDCl}_{3}\right)$

$\begin{array}{ll}7.45 & 7.35\end{array}$

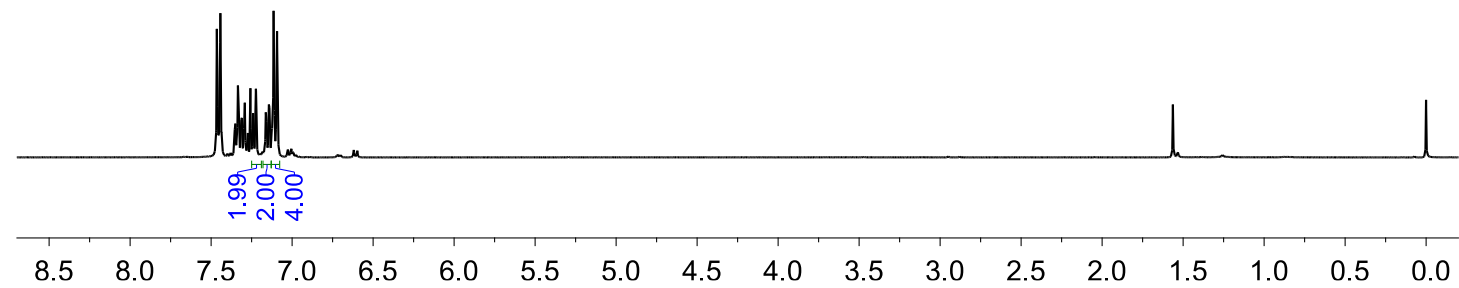

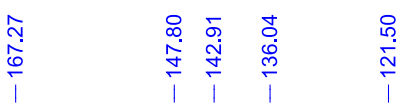

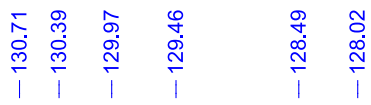
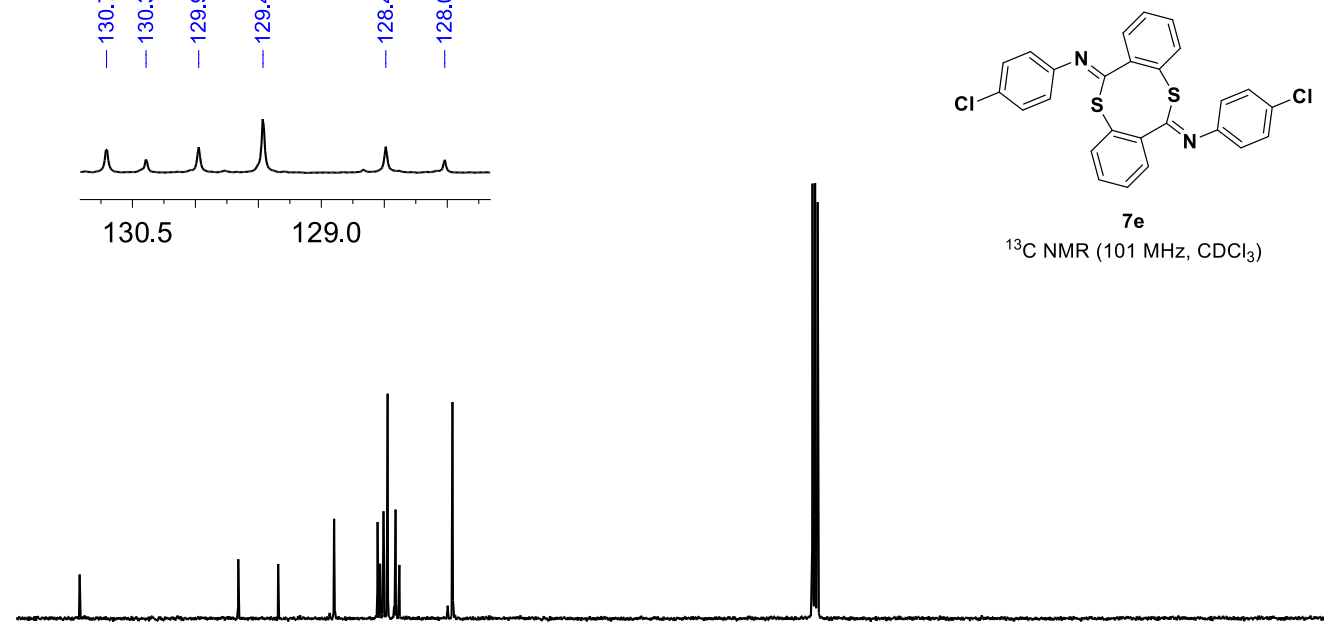

${ }^{13} \mathrm{C}$ NMR $\left(101 \mathrm{MHz}, \mathrm{CDCl}_{3}\right)$

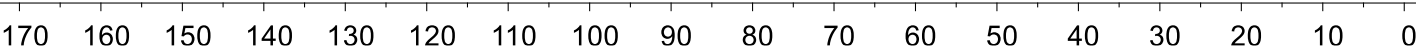




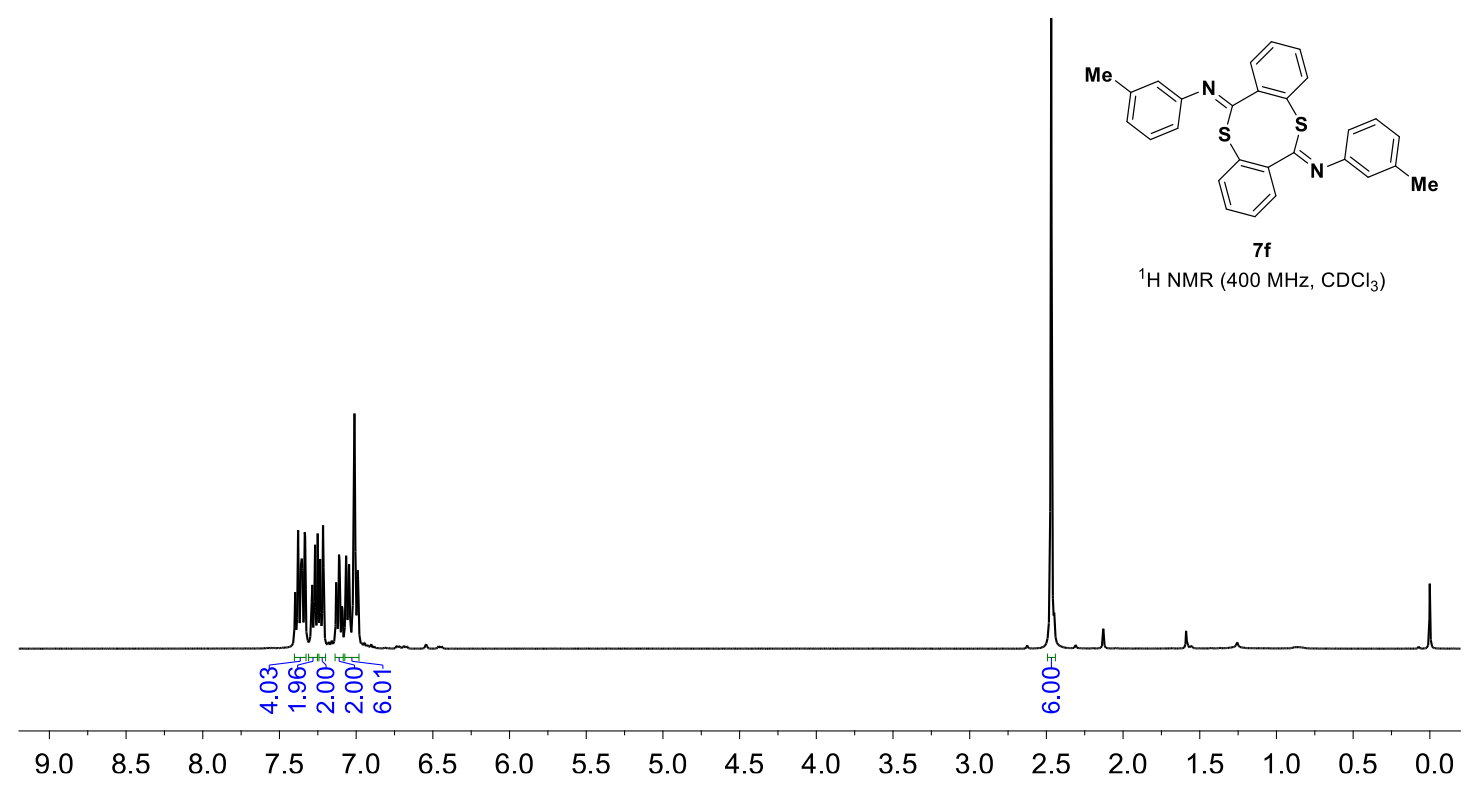

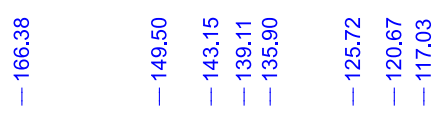

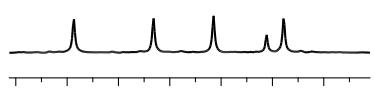

129.5

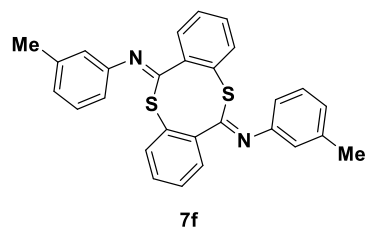

$\left.{ }^{13} \mathrm{C} \mathrm{NMR} \mathrm{(101} \mathrm{MHz,} \mathrm{CDCl}_{3}\right)$

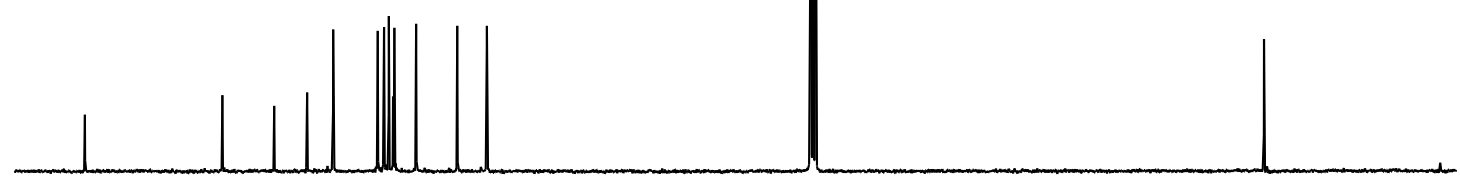

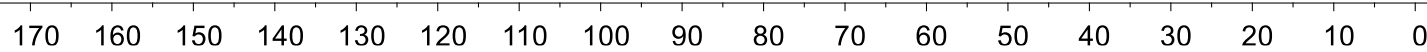



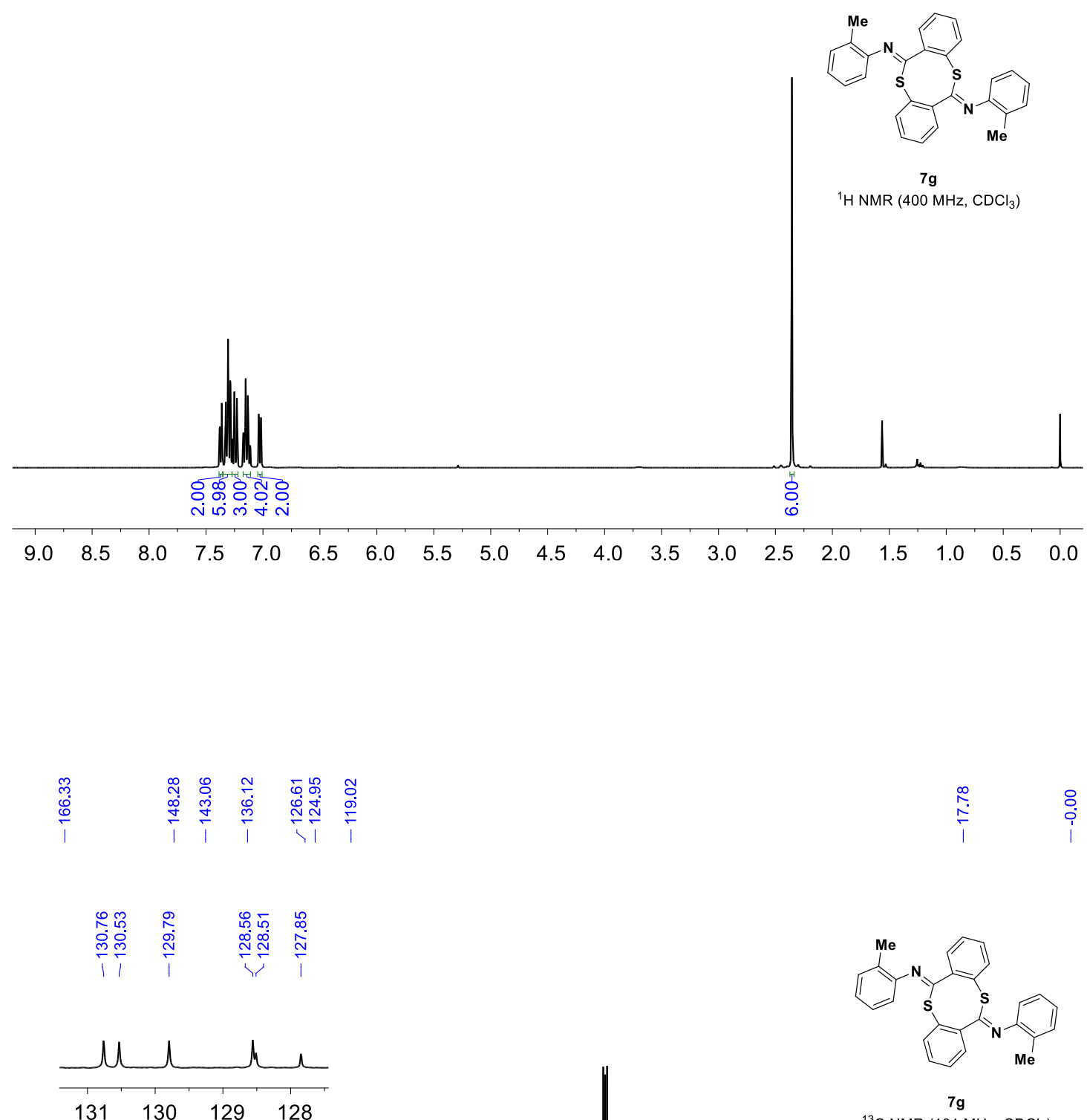

$\begin{array}{llll}131 & 130 & 129 & 128\end{array}$

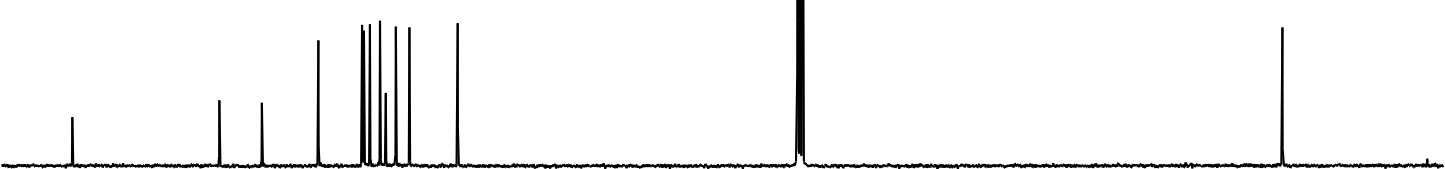

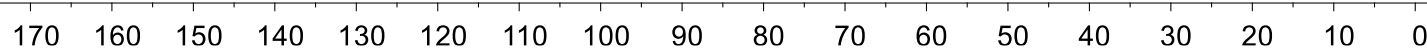



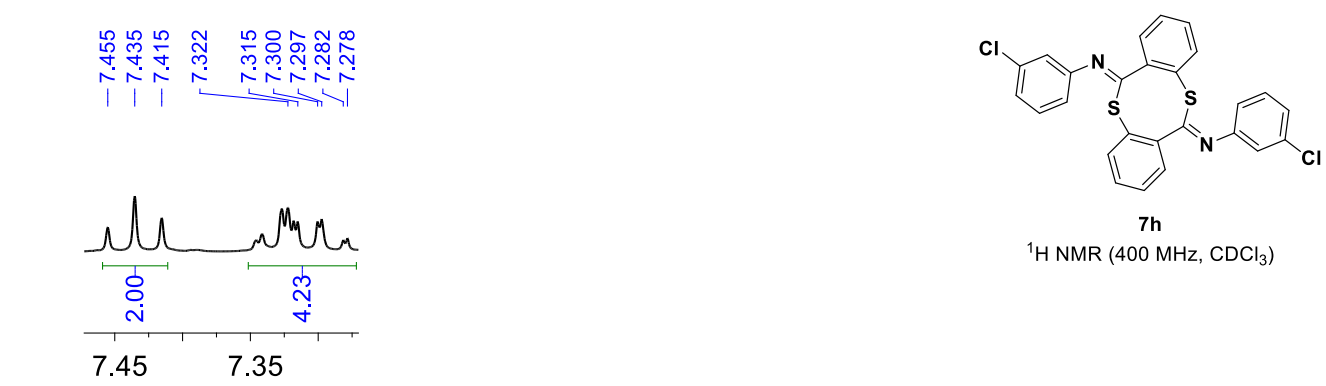

${ }^{1} \mathrm{H} \mathrm{NMR}\left(400 \mathrm{MHz}, \mathrm{CDCl}_{3}\right)$

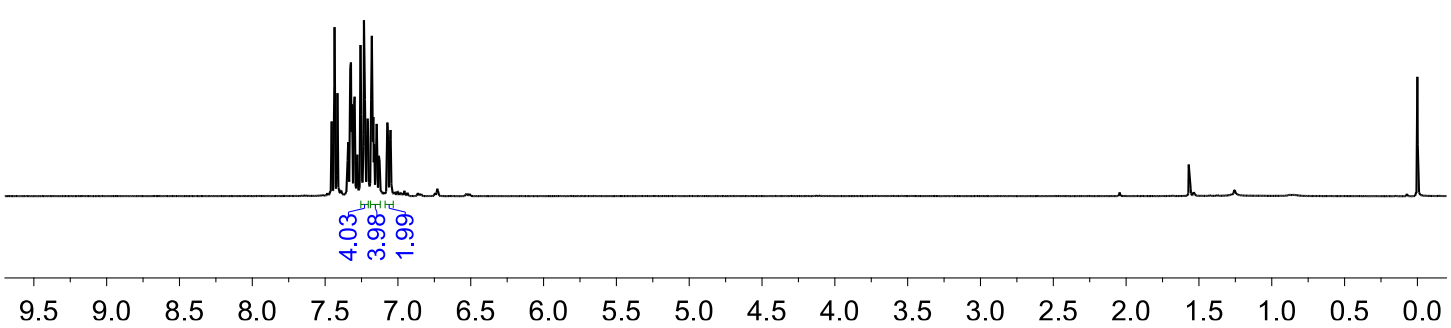

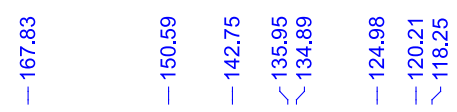
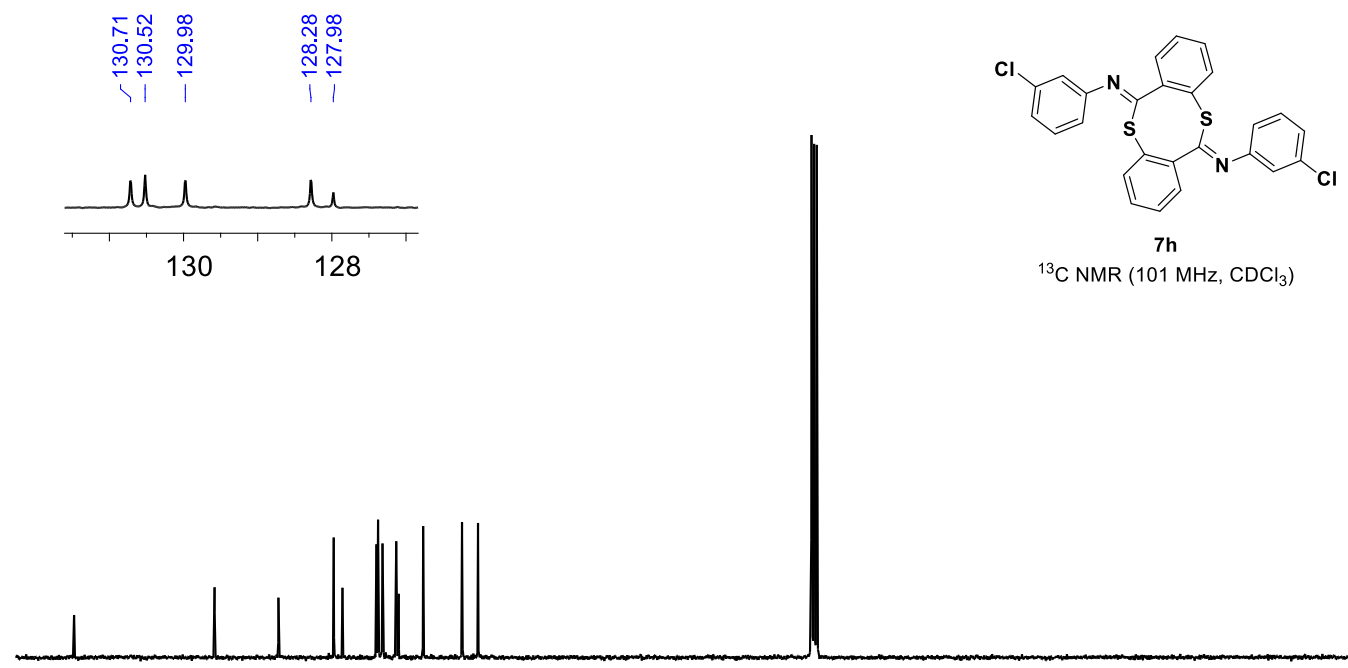

${ }^{13} \mathrm{C} \mathrm{NMR}\left(101 \mathrm{MHz}, \mathrm{CDCl}_{3}\right)$

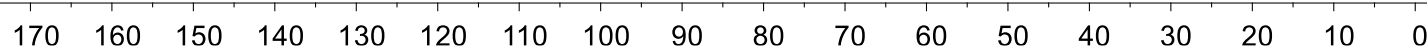




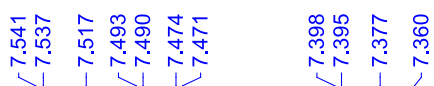

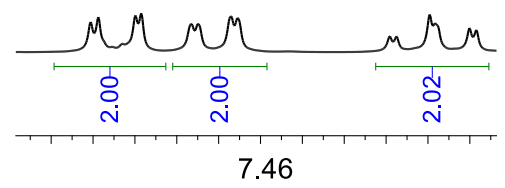

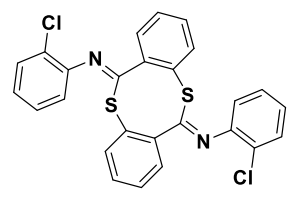

$7 \mathbf{i}$

${ }^{1} \mathrm{H} \mathrm{NMR}\left(400 \mathrm{MHz}, \mathrm{CDCl}_{3}\right)$

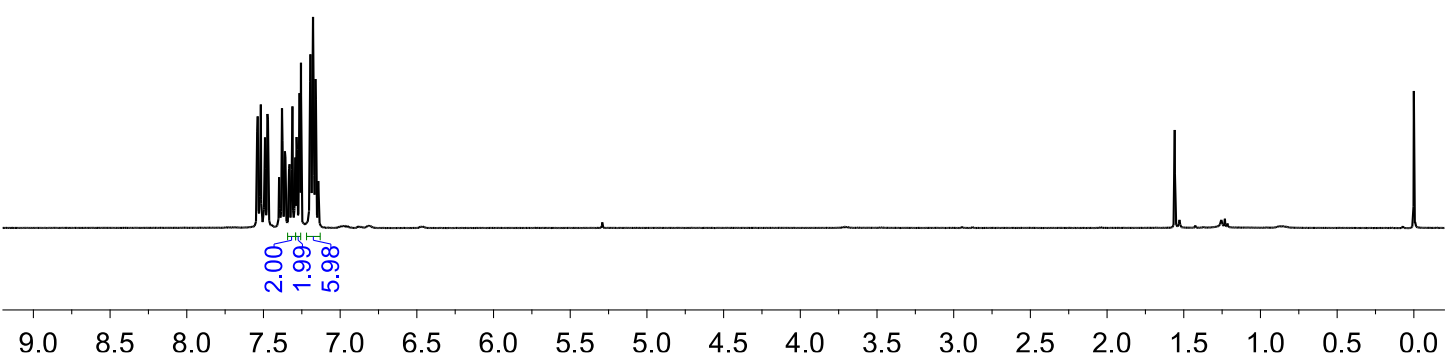

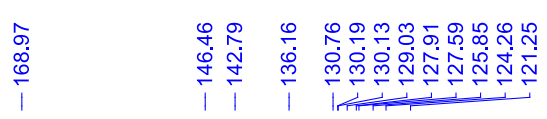

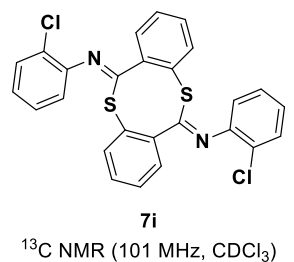

${ }^{13} \mathrm{C}$ NMR $\left(101 \mathrm{MHz}, \mathrm{CDCl}_{3}\right)$

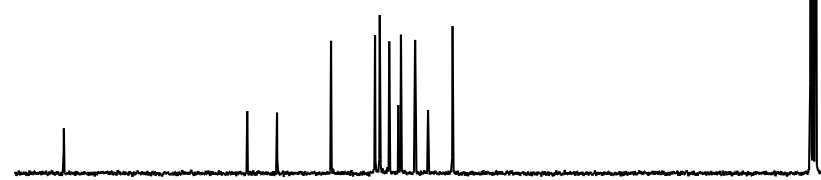

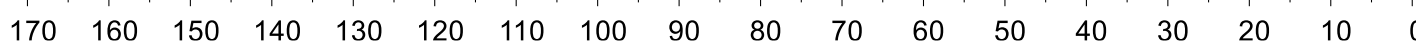




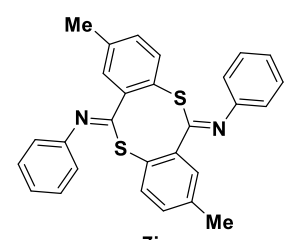

${ }^{1} \mathrm{H}$ NMR $\left(400 \mathrm{MHz}, \mathrm{CDCl}_{3}\right)$

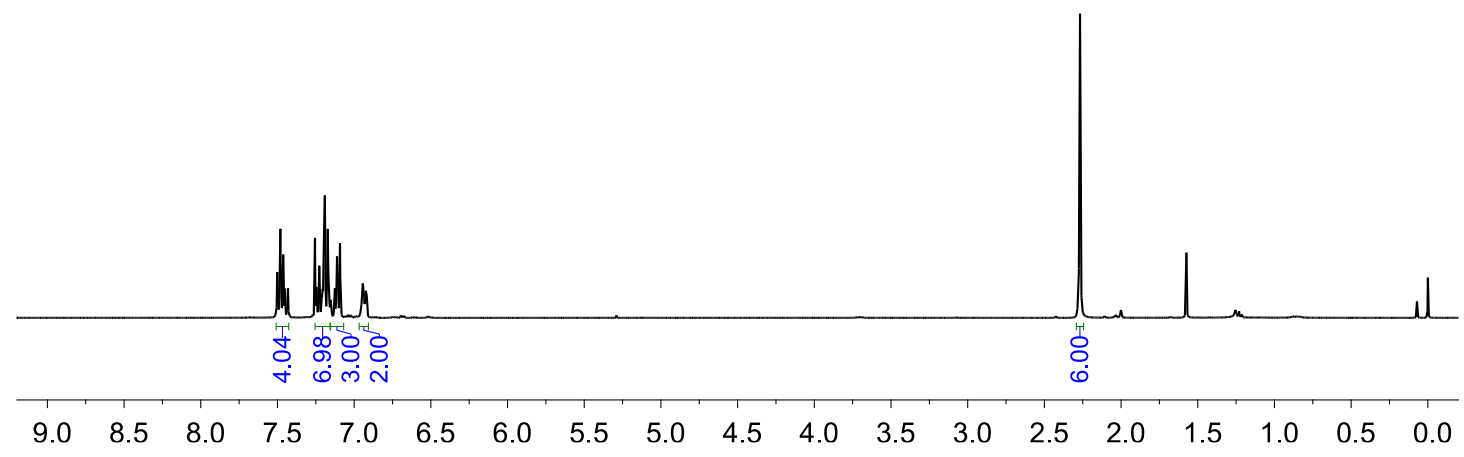

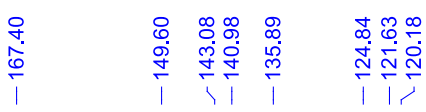

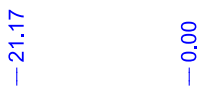

i
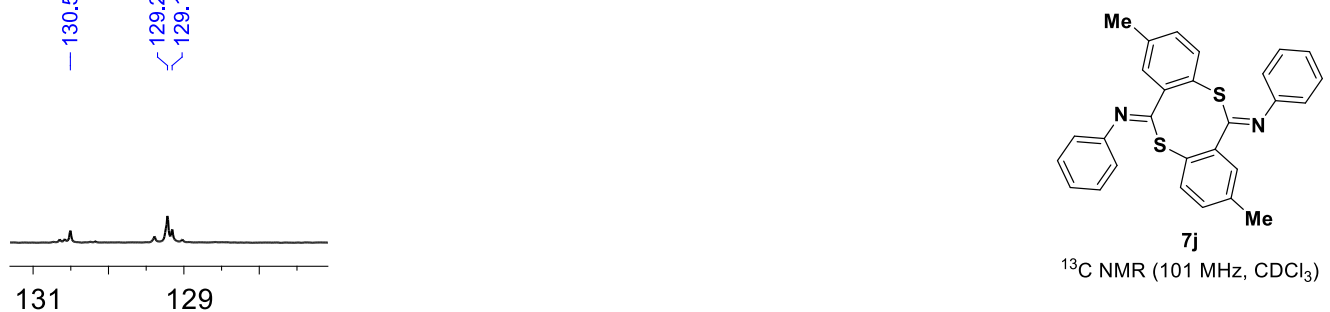

${ }^{13} \mathrm{C}$ NMR $\left(101 \mathrm{MHz}, \mathrm{CDCl}_{3}\right)$

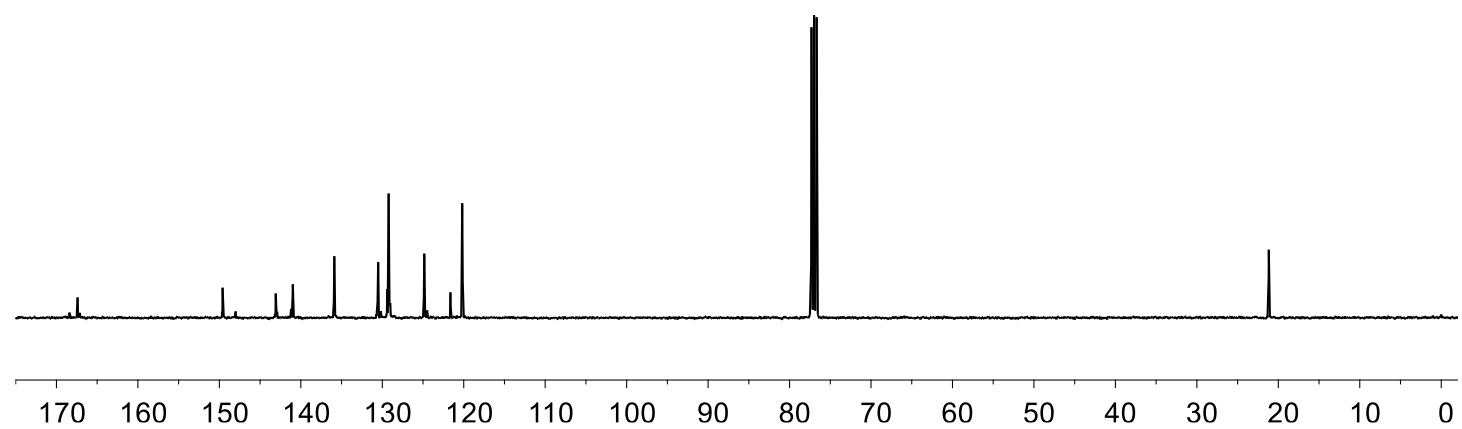




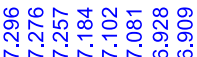

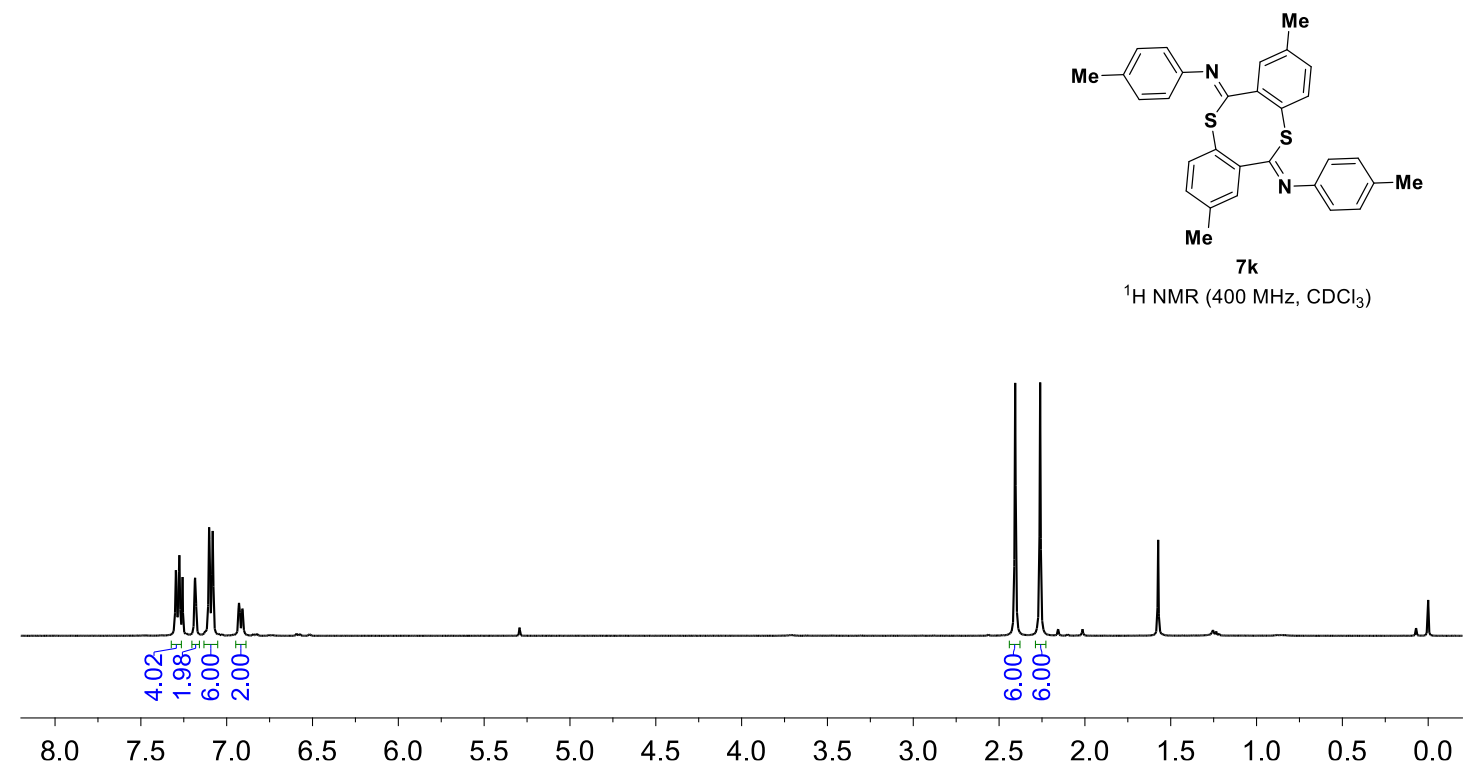

${ }^{1} \mathrm{H}$ NMR $\left(400 \mathrm{MHz}, \mathrm{CDCl}_{3}\right)$

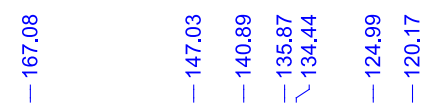

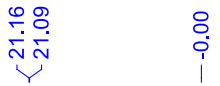

$\frac{\mathfrak{N}}{\stackrel{丶}{+}}$

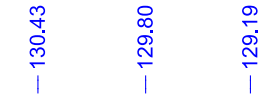
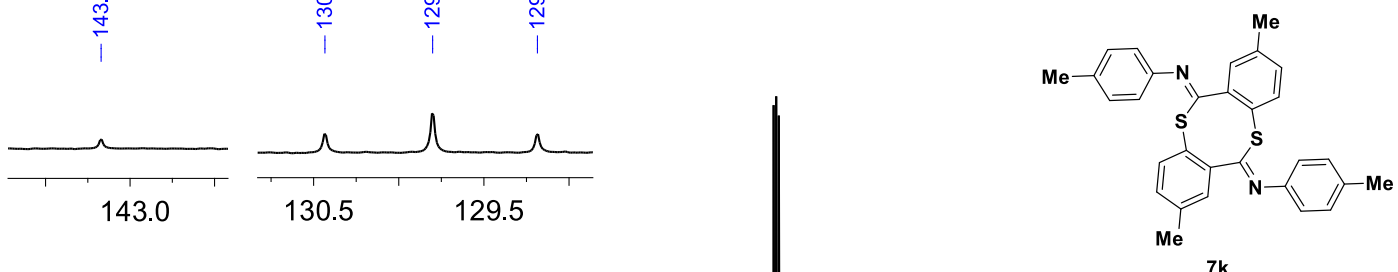

${ }^{13} \mathrm{C} \mathrm{NMR}\left(101 \mathrm{MHz}, \mathrm{CDCl}_{3}\right)$

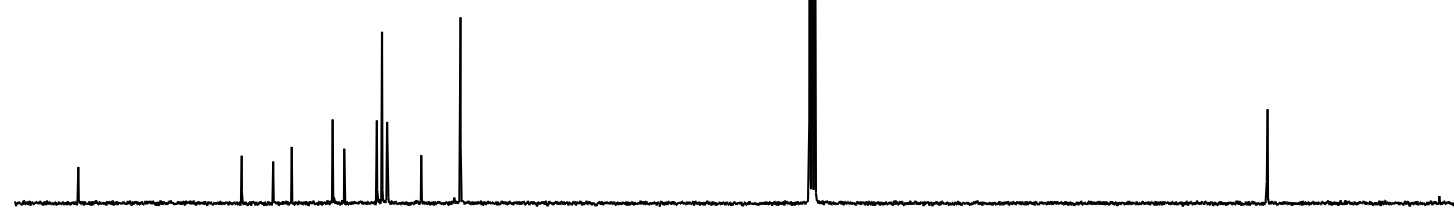

$\begin{array}{llllllllllllllllll}170 & 160 & 150 & 140 & 130 & 120 & 110 & 100 & 90 & 80 & 70 & 60 & 50 & 40 & 30 & 20 & 10 & 0\end{array}$ 


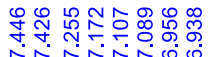

NNNNNG0

1

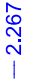

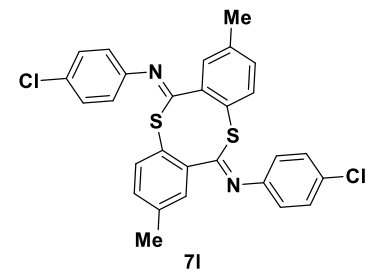

${ }^{1} \mathrm{H} \mathrm{NMR}\left(400 \mathrm{MHz}, \mathrm{CDCl}_{3}\right)$

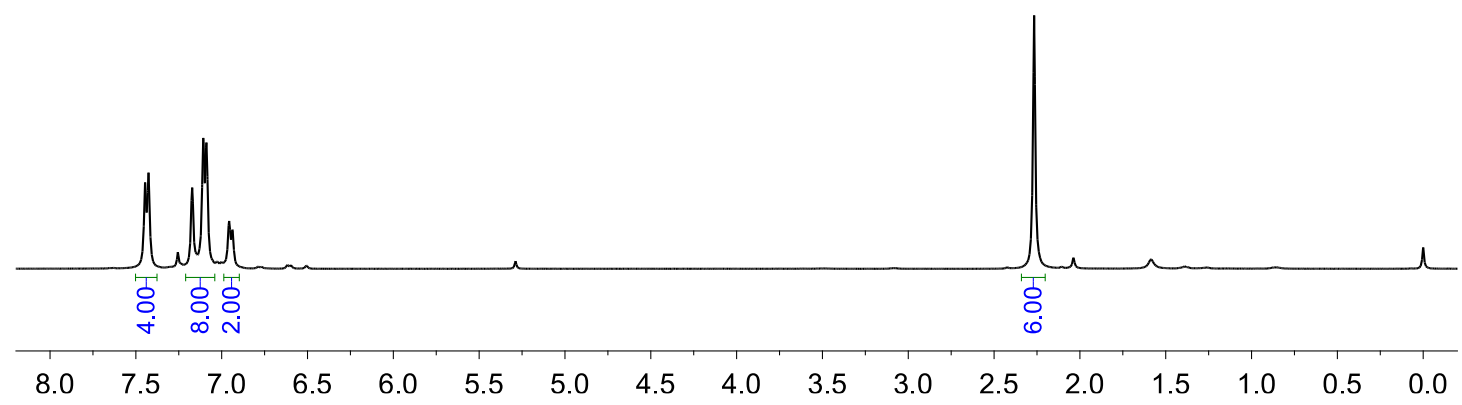

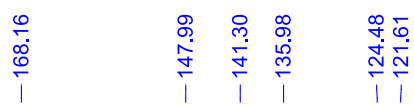

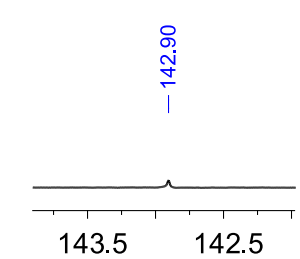

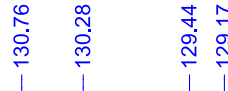
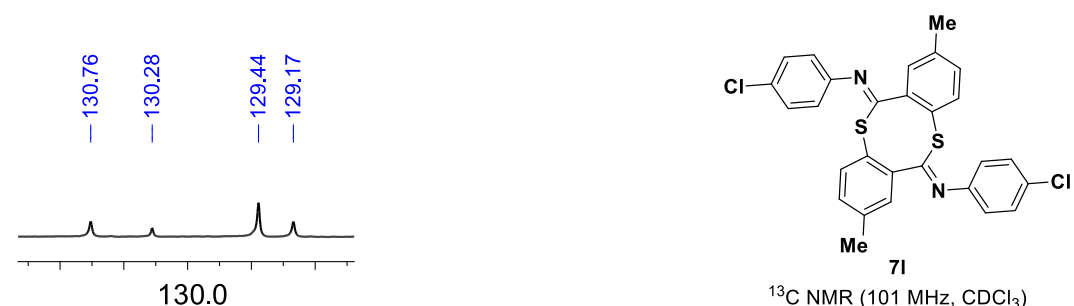

$\left.{ }^{13} \mathrm{C} \mathrm{NMR} \mathrm{(101} \mathrm{MHz,} \mathrm{CDCl}_{3}\right)$

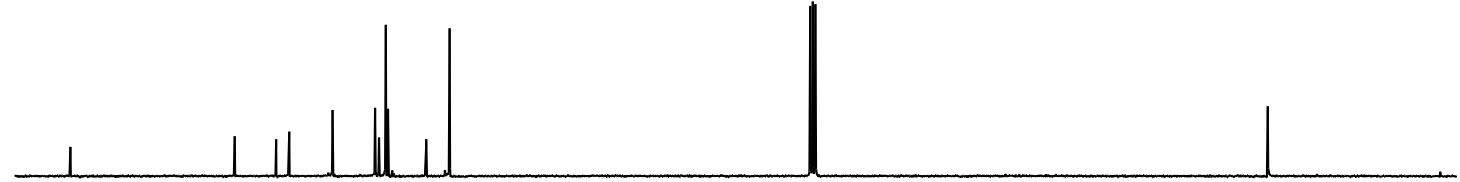

$\begin{array}{llllllllllllllllll}170 & 160 & 150 & 140 & 130 & 120 & 110 & 100 & 90 & 80 & 70 & 60 & 50 & 40 & 30 & 20 & 10 & 0\end{array}$ 


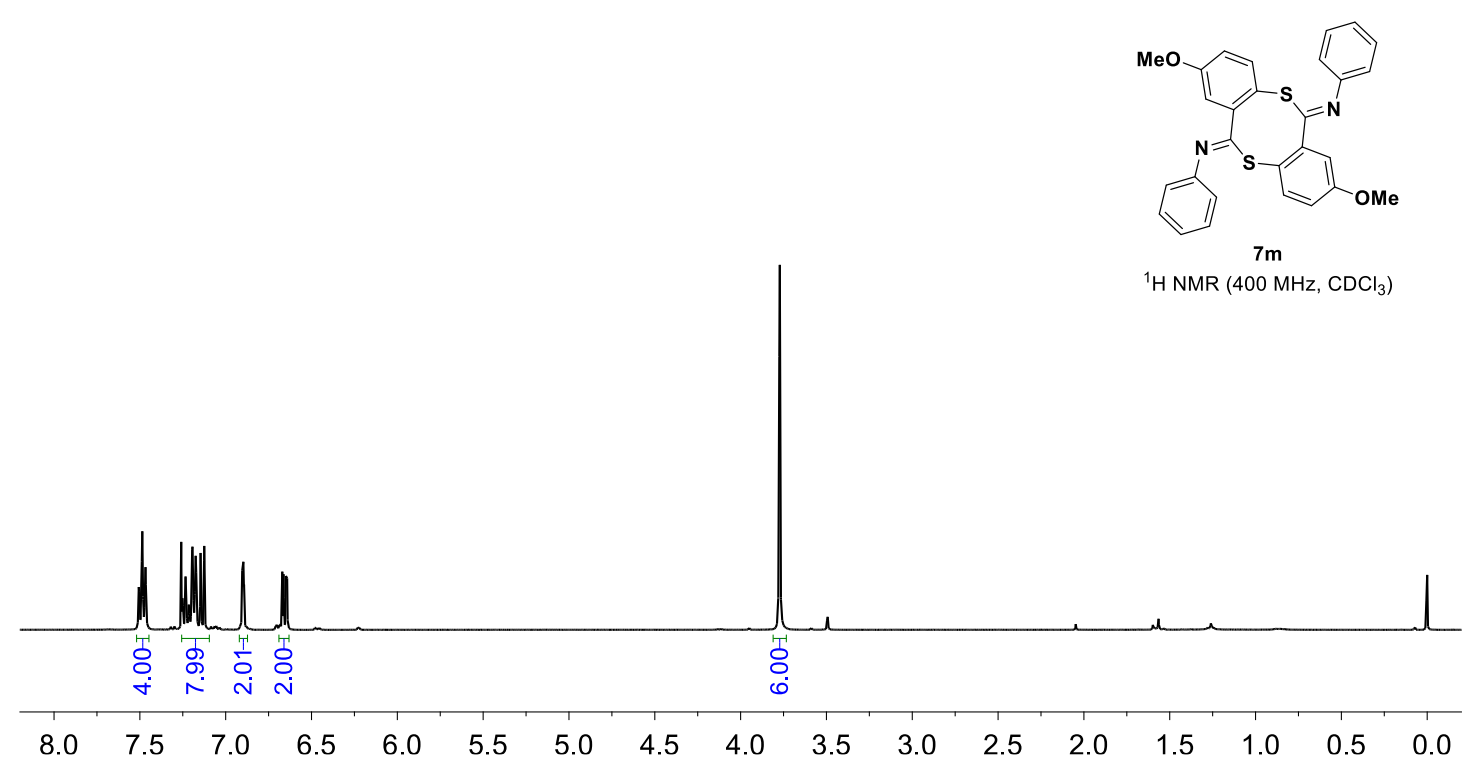

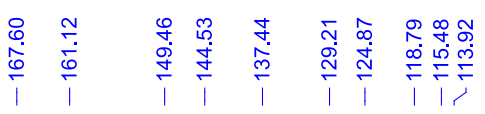

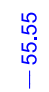
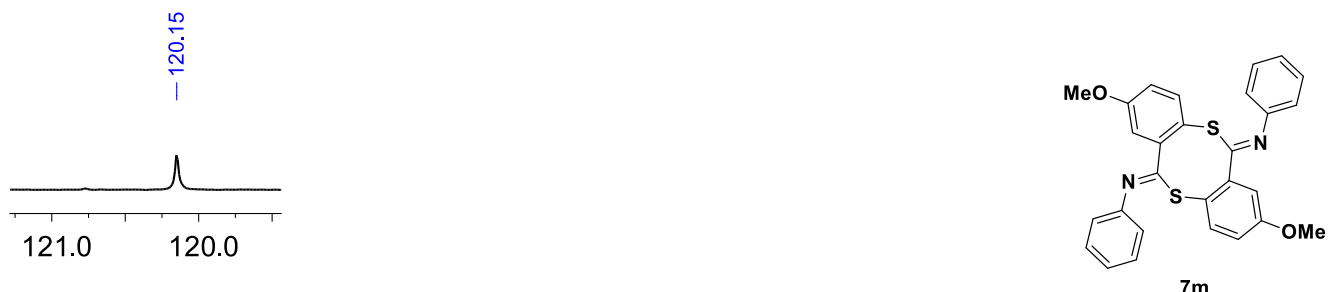

${ }^{13} \mathrm{C}$ NMR $\left(101 \mathrm{MHz}, \mathrm{CDCl}_{3}\right)$

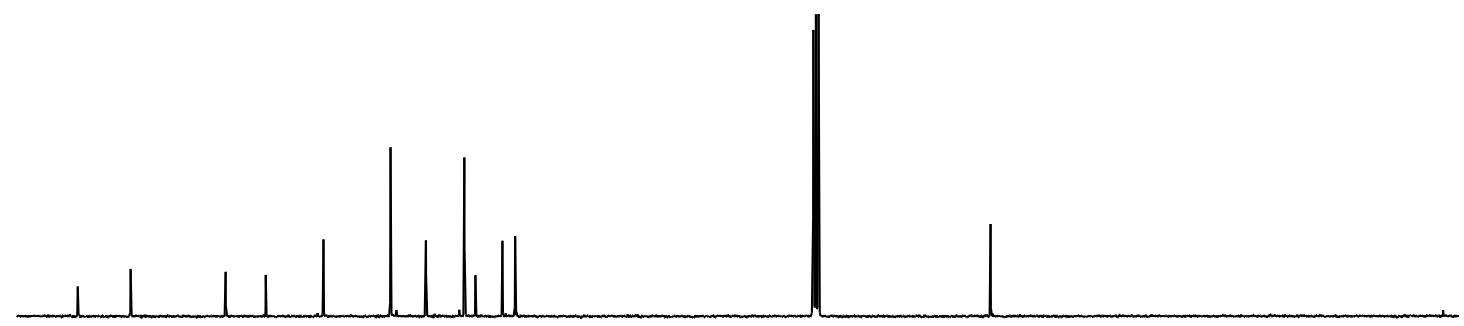

$\begin{array}{llllllllllllllllll}170 & 160 & 150 & 140 & 130 & 120 & 110 & 100 & 90 & 80 & 70 & 60 & 50 & 40 & 30 & 20 & 10 & 0\end{array}$ 


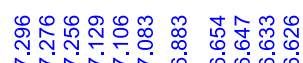 \\ Nrintiogogo}

$\underset{\substack{1 \\ 0}}{1}$

:

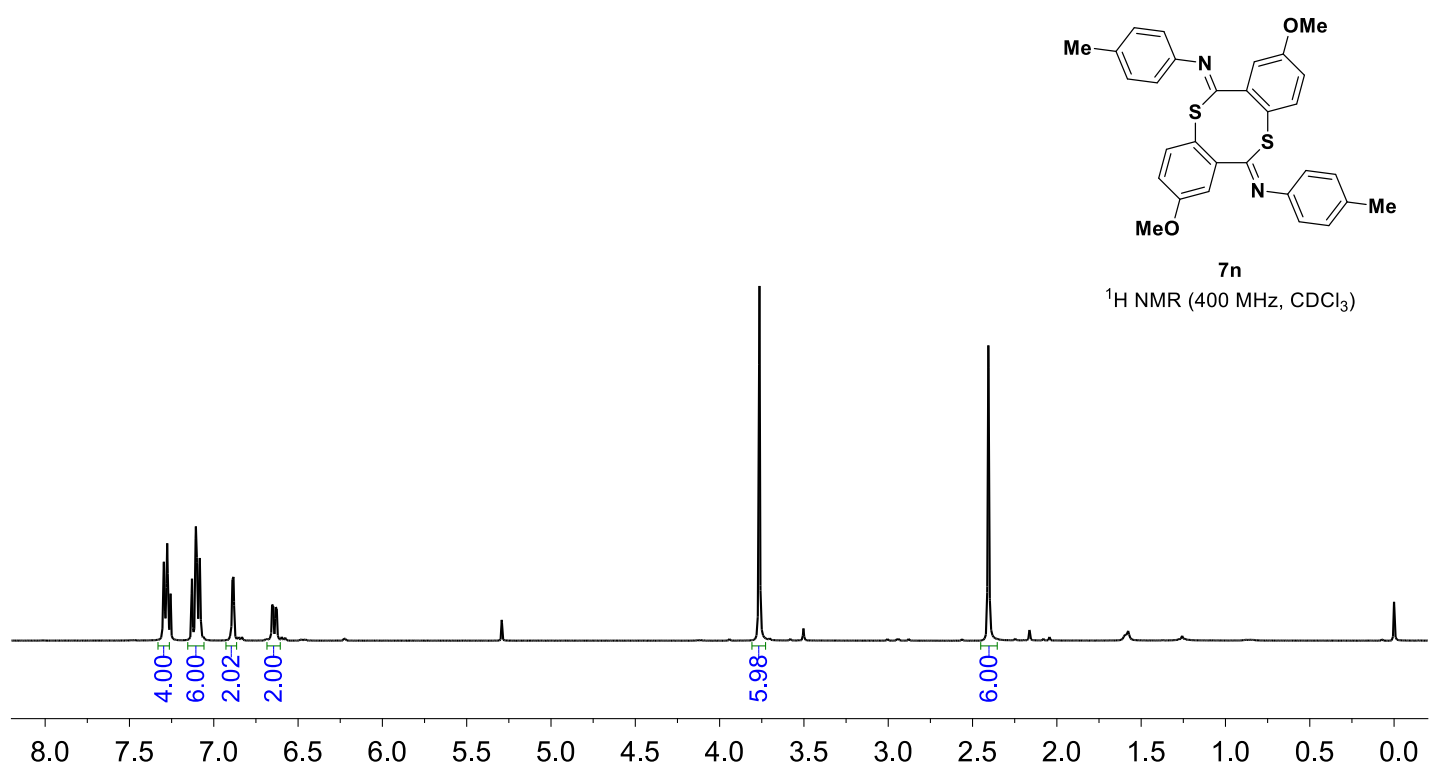

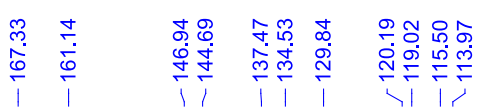

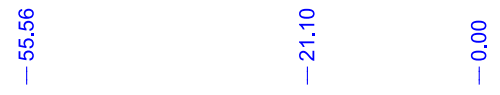

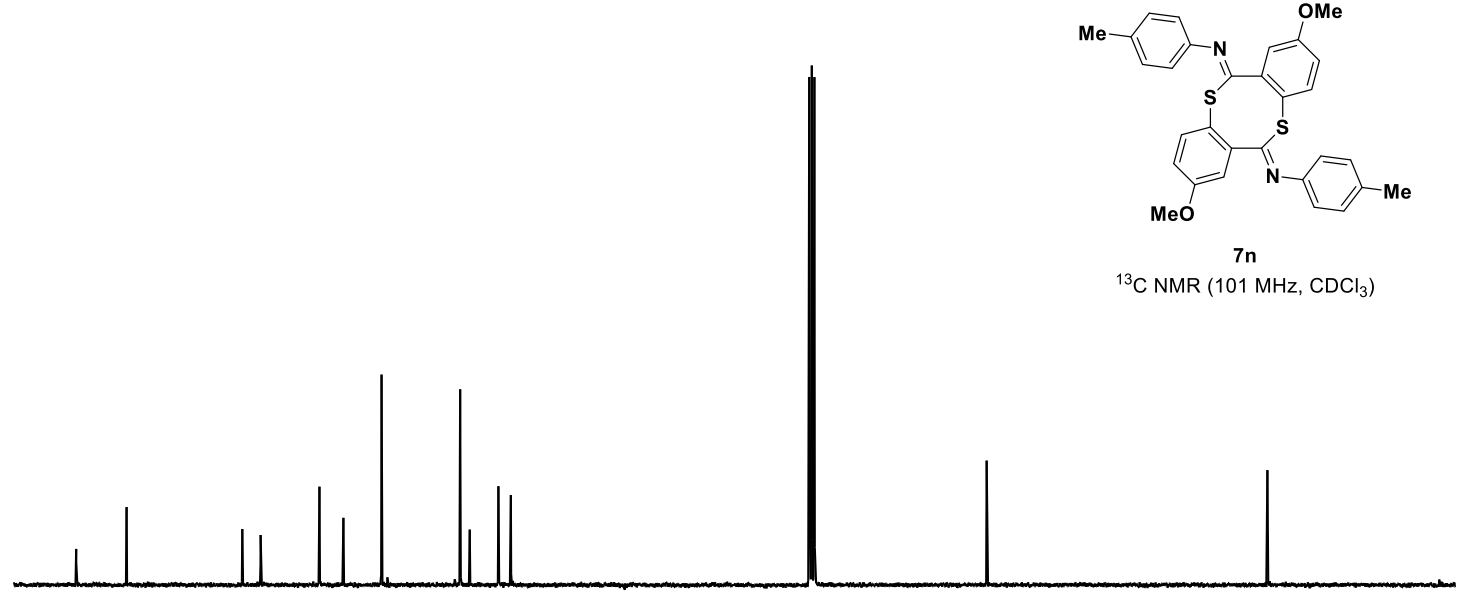

$\begin{array}{lllllllllllllllll}170 & 160 & 150 & 140 & 130 & 120 & 110 & 100 & 90 & 80 & 70 & 60 & 50 & 40 & 30 & 20 & 10\end{array}$ 

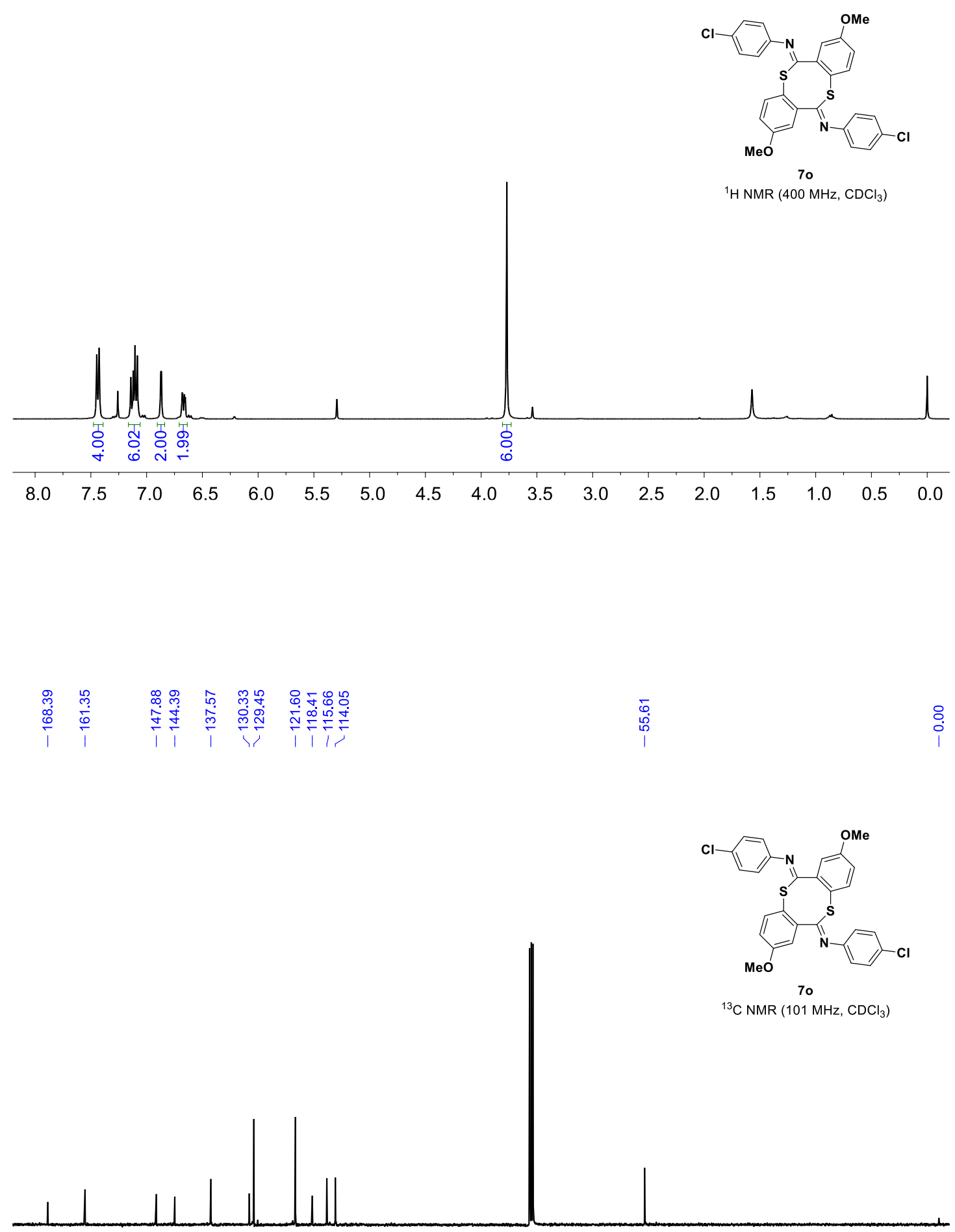

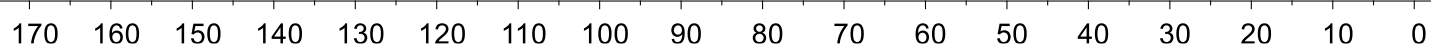




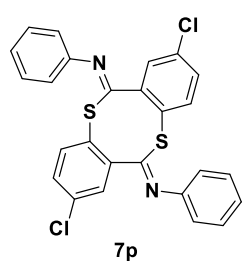

${ }^{1} \mathrm{H} \mathrm{NMR}\left(400 \mathrm{MHz}, \mathrm{CDCl}_{3}\right)$

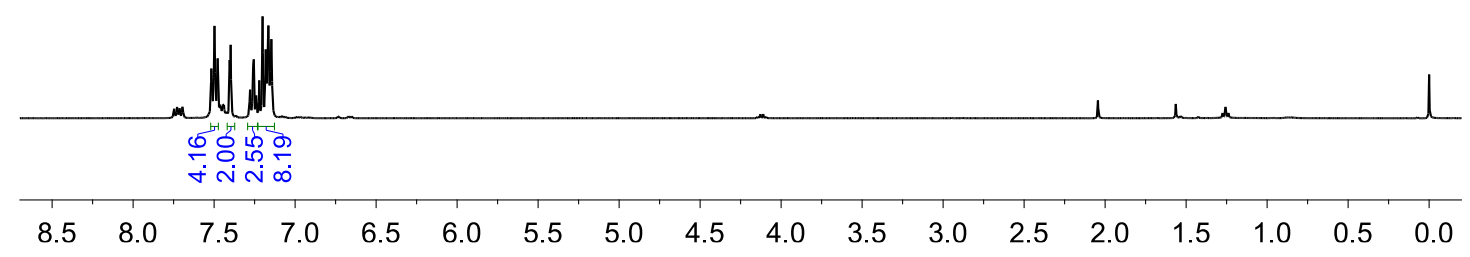

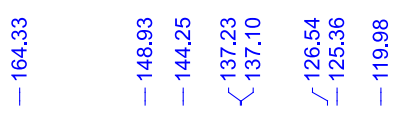

$\begin{array}{lll}\uparrow & \stackrel{0}{0} & \infty \\ \stackrel{0}{0} & \stackrel{\infty}{\sim} & \stackrel{\infty}{\leftarrow} \\ i & i & i\end{array}$
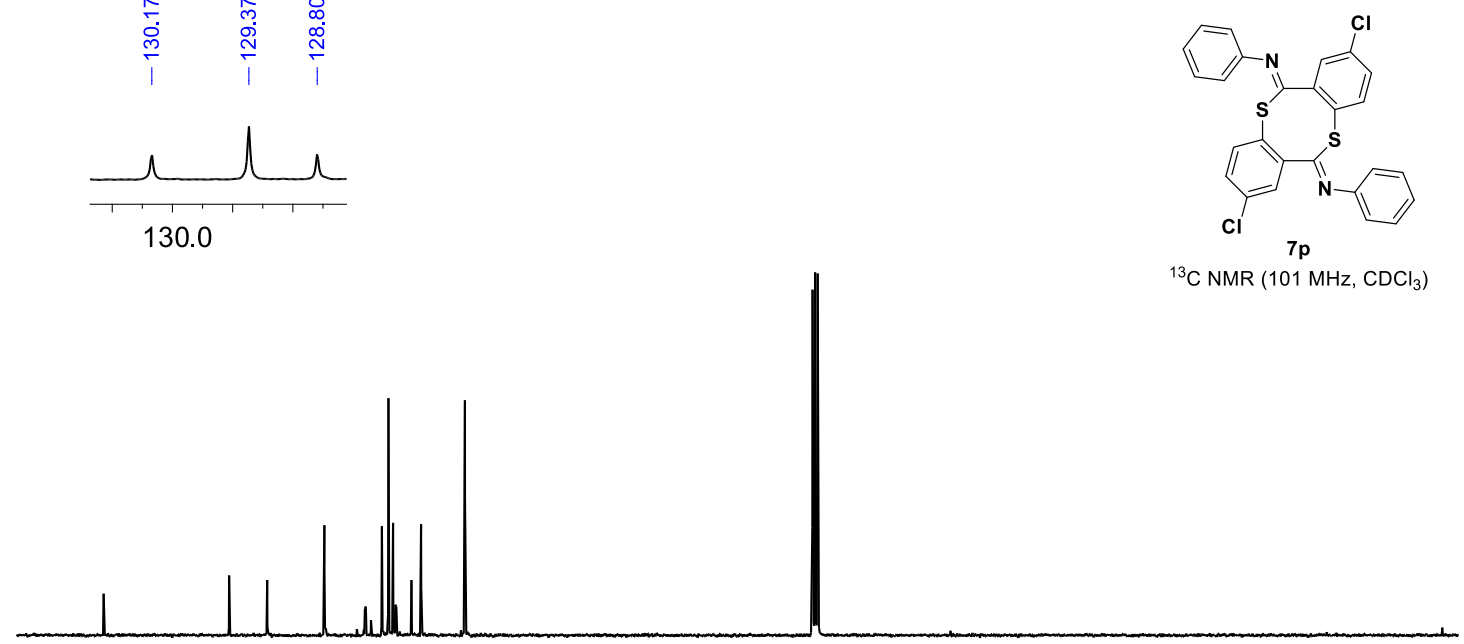

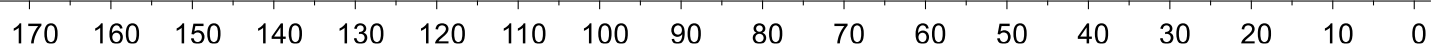




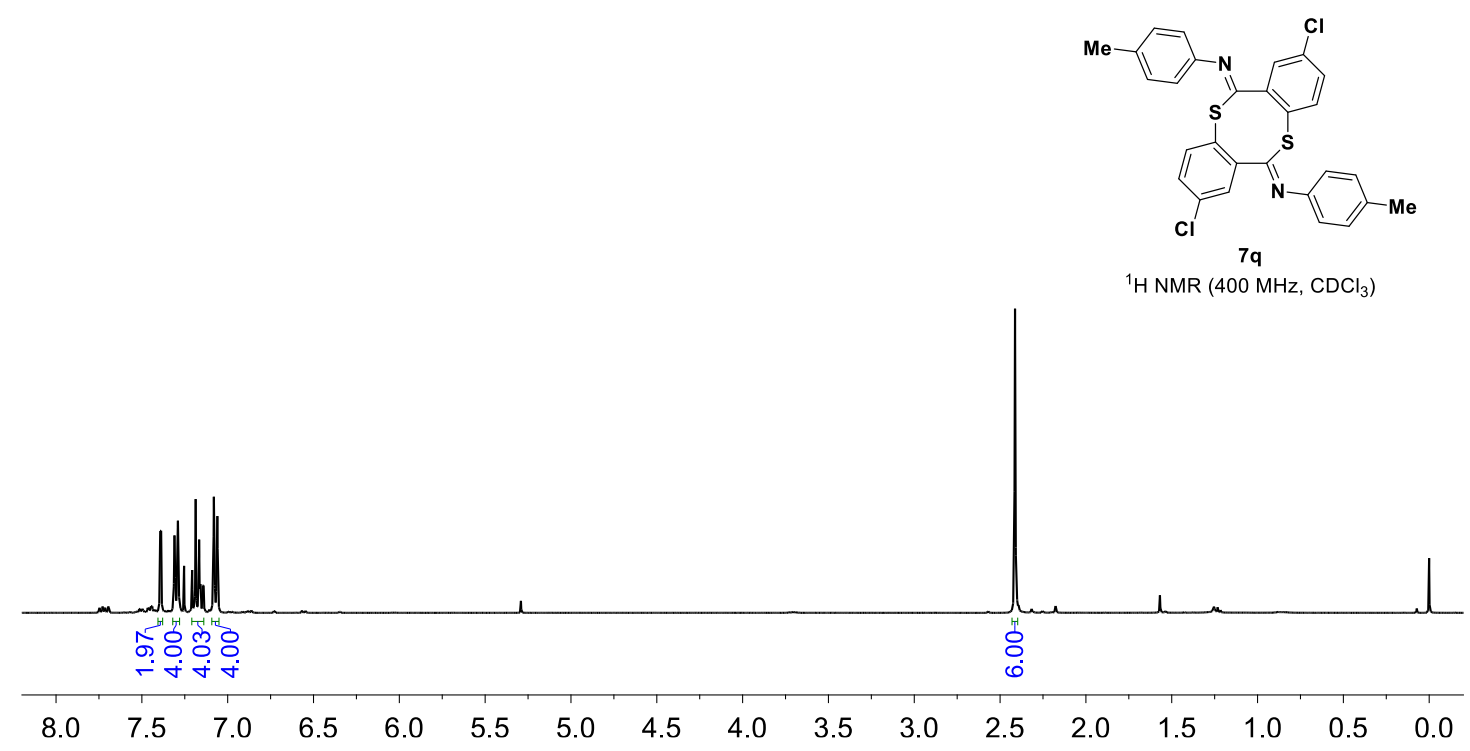

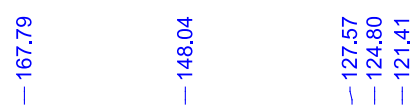
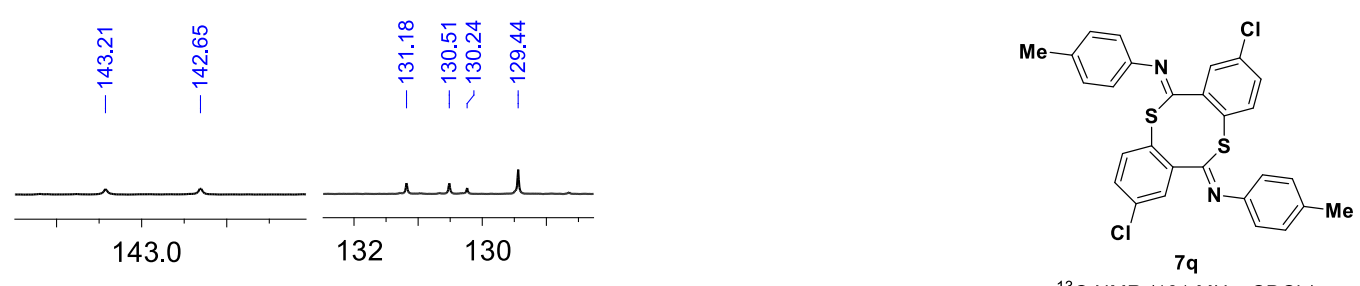

${ }^{13} \mathrm{C}$ NMR $\left(101 \mathrm{MHz}, \mathrm{CDCl}_{3}\right)$

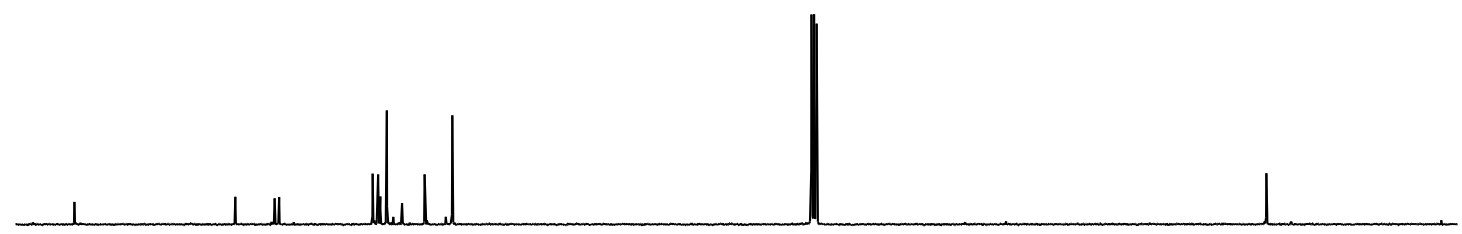

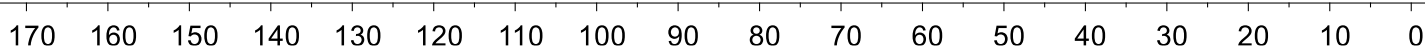




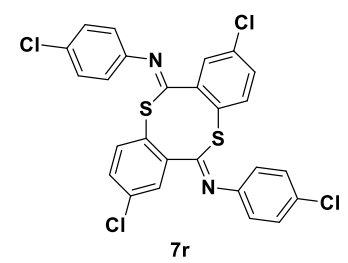

${ }^{1} \mathrm{H}$ NMR $\left(400 \mathrm{MHz}, \mathrm{CDCl}_{3}\right)$

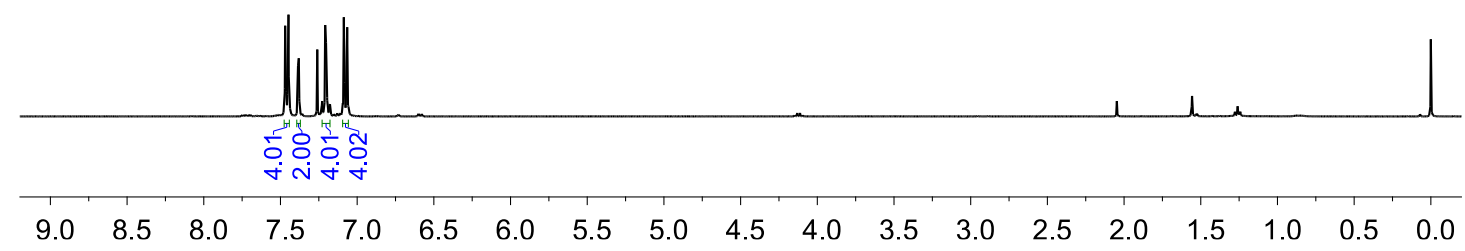

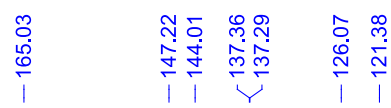
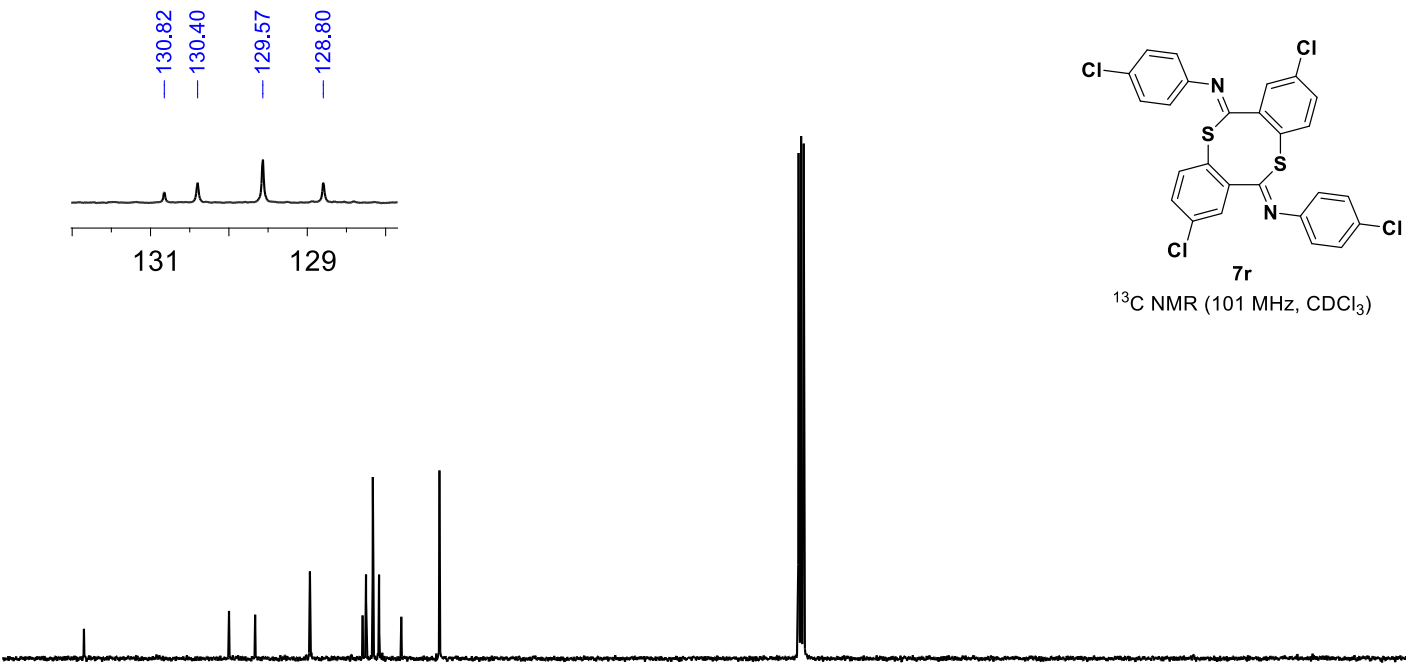

${ }^{13} \mathrm{C} \mathrm{NMR}\left(101 \mathrm{MHz}, \mathrm{CDCl}_{3}\right)$

$\begin{array}{llllllllllllllllll}170 & 160 & 150 & 140 & 130 & 120 & 110 & 100 & 90 & 80 & 70 & 60 & 50 & 40 & 30 & 20 & 10 & 0\end{array}$ 


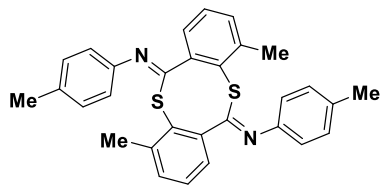

$7 \mathrm{~s}$

${ }^{1} \mathrm{H}$ NMR $\left(400 \mathrm{MHz}, \mathrm{CDCl}_{3}\right)$

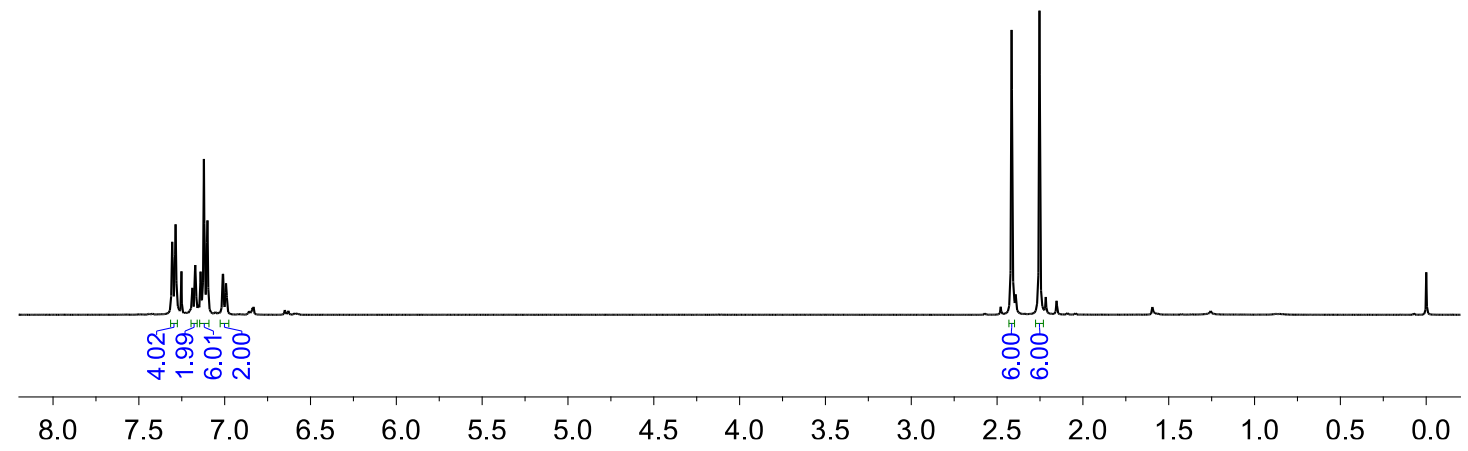

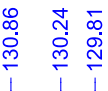
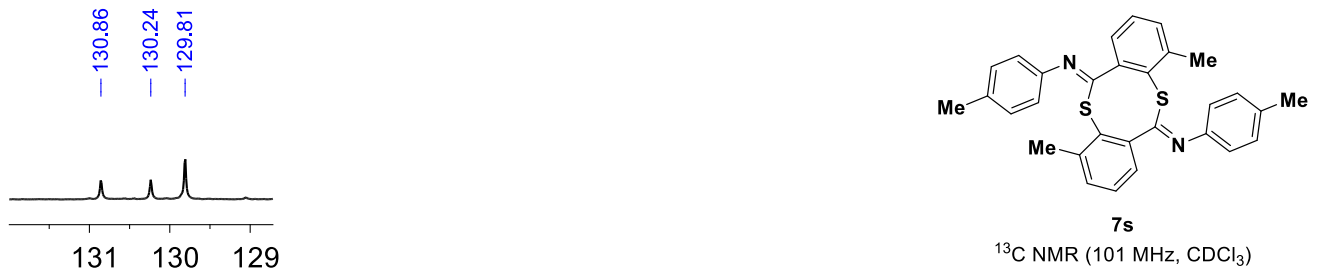

${ }^{13} \mathrm{C}$ NMR $\left(101 \mathrm{MHz}, \mathrm{CDCl}_{3}\right)$

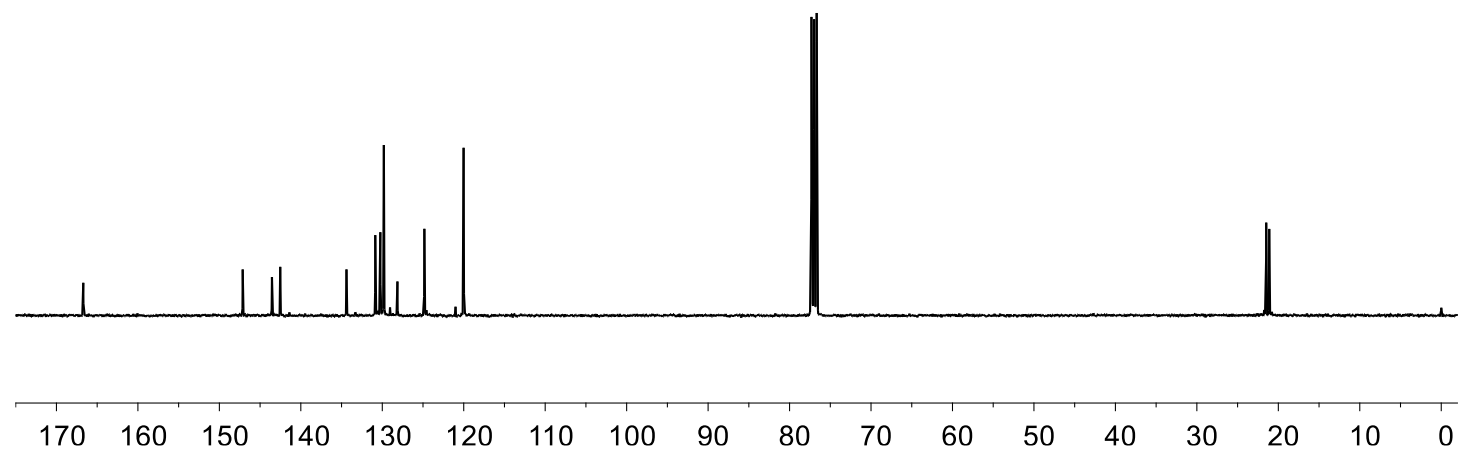




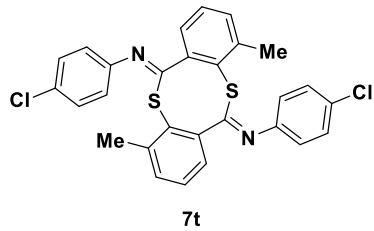

${ }^{1} \mathrm{H} \mathrm{NMR}\left(400 \mathrm{MHz}, \mathrm{CDCl}_{3}\right)$
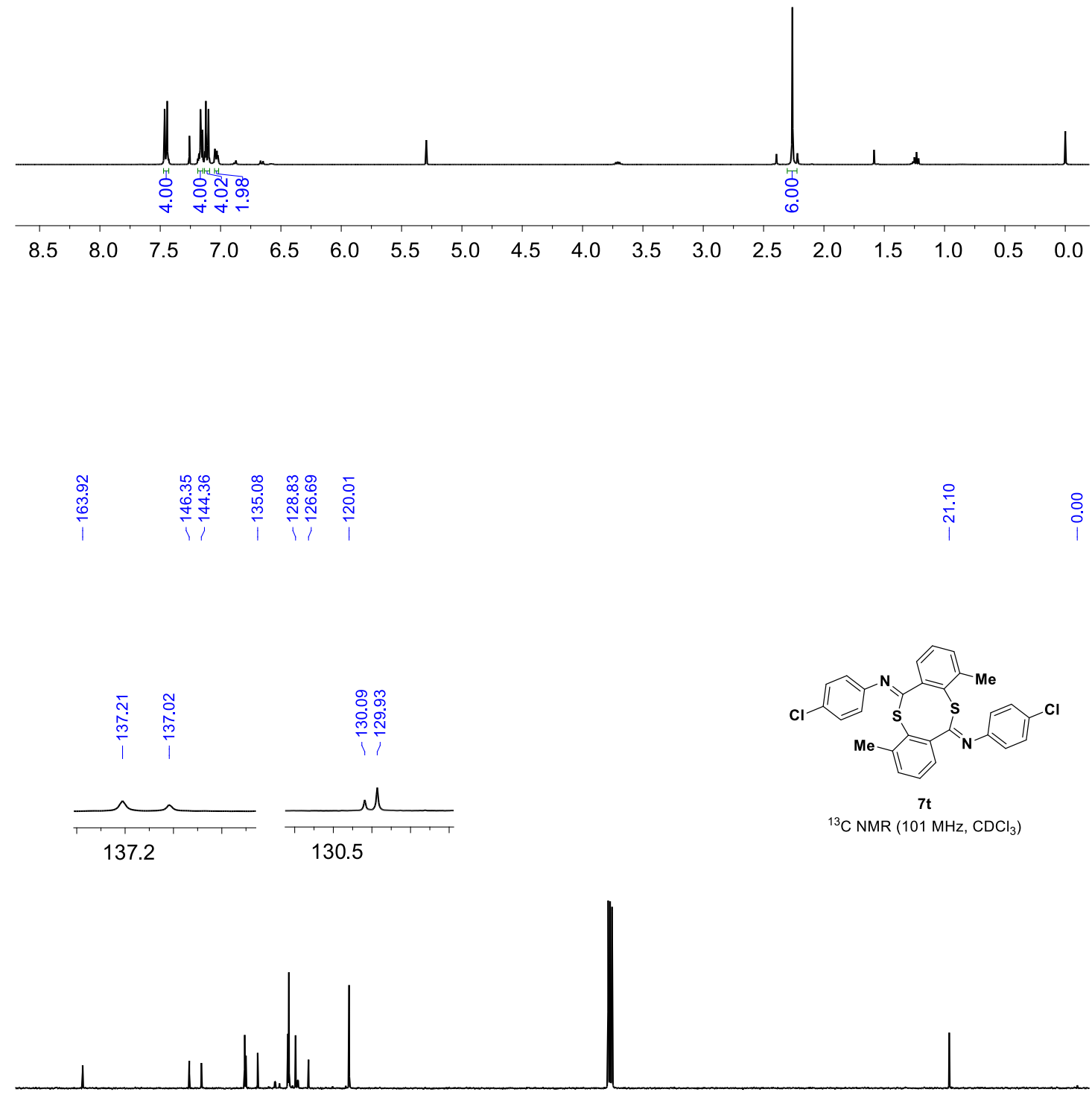

$\begin{array}{lllllllllllllllllll}170 & 160 & 150 & 140 & 130 & 120 & 110 & 100 & 90 & 80 & 70 & 60 & 50 & 40 & 30 & 20 & 10 & 0\end{array}$ 


\section{References}

(1) (a) Xu, Z.-M.; Li, H.-X.; Young, D. J.; Zhu, D.-L.; Li, H.-Y.; Lang, J.-P. Exogenous Photosensitizer-, Metal-, and Base-Free Visible-Light-Promoted $\mathrm{C}-\mathrm{H}$ Thiolation via Reverse Hydrogen Atom Transfer. Org. Lett. 2019, 21, 237-241. (b) Ma, Y.-G.; Huang, M.-Q.; Liu, Z.; Liu, J.-Q.; Wang, X.-S. Synthesis of Substituted $4 \mathrm{H}$-Thiochromen-4-imines via Copper-Catalyzed Cyclization Cascades of o-Bromobenzothioamides with Terminal Alkynes. J. Org. Chem. 2018, 83, 9504-9509.

(2) (a) Sheldrick, G. M. SHELXS-97, Program for Solution of Crystal Structures, University of Gottingen, Germany, 1997. (b) Sheldrick, G. M. SHELXL-97, Program for Refinement of Crystal Structures, University of Gottingen, Germany, 1997.

(3) Soria-Castro, S. M.; Peñéñory, A. B. Efficient Cu-Catalyzed Base-Free C-S Coupling under Conventional and Microwave Heating. A Simple Access to S-Heterocycles and Sulfides. Beilstein J. Org. Chem. 2013, 9, 467-475.

(4) Mitra, K.; Pohl, M. E.; MacGillivray, L. R.; Barnes, C. L.; Gates, K. S. Synthesis and Structure of Functionalized Derivatives of the Cleft-Shaped Molecule Dithiosalicylide. J. Org. Chem. 1997, 62, 9361-9364.

(5) Hortmann, A. G.; Aron, A. J.; Bhattacharya, A. K. 3H-1,2-Benzodithiole Oxides: Studies Directed toward the Generation of $o$-Thiobenzoquinone Methide and Benzo[b]thiete. J. Org. Chem. 1978, 43, 3374-3378.

(6) Iyer, R. P.; Phillips, L. R.; Egan, W.; Regan, J. B.; Beaucage, S. L. The Automated Synthesis of Sulfur-Containing Oligodeoxyribonucleotides using $3 \mathrm{H}$-1,2-Benzodithiol-3-one 1,1-Dioxide as a Sulfur-Transfer Reagent. J. Org. Chem. 1990, 55, 4693-4699. 